Novel strategies for risk assessment of pyrrolizidine alkaloids

Lu Chen 


\section{Thesis committee}

\section{Promotor}

Prof. Dr I.M.C.M. Rietjens

Professor of Toxicology

Wageningen University \& Research

\section{Co-promotor}

Dr A.A.C.M. Peijnenburg

Wageningen Food Safety Research

\section{Other members}

Prof. Dr R.F. Witkamp, Wageningen University \& Research

Prof. R. Gehring, University Utrecht

Dr J. van Benthem, RIVM, Bilthoven

Dr S. Jeurissen, RIVM, Bilthoven

This research was conducted under the auspices of the Graduate School VLAG (Advanced Studies in Food Technology, Agrobiotechnology, Nutrition and Health Sciences) 


\title{
Novel strategies for risk assessment of pyrrolizidine alkaloids
}

\author{
Lu Chen
}

\section{Thesis}

submitted in fulfilment of the requirements for the degree of doctor at Wageningen University by the authority of the Rector Magnificus Prof. Dr A. P.J. Mol, in the presence of the

Thesis Committee appointed by the Academic Board to be defended in public on Friday 6 September 2019 at 11.00 a.m. in the Aula. 


\section{Lu Chen}

Novel strategies for risk assessment of pyrrolizidine alkaloids, 243 pages.

$\mathrm{PhD}$ thesis, Wageningen University, Wageningen, the Netherlands (2019) With references, with summary in English

ISBN: 978-94-6395-037-4

DOI: https://doi.org/10.18174/496159 


\section{Table of Contents}

Chapter 1 General introduction 7

Chapter 2 Risk assessment for pyrrolizidine alkaloids detected in (herbal) teas and 27 plant food supplements

Chapter 3 Risk assessment of intake of pyrrolizidine alkaloids from herbal teas and $\quad \mathbf{6 1}$ medicines following realistic exposure scenarios

Chapter 4 Use of physiologically based kinetic modelling-facilitated reverse dosimetry to convert in vitro cytotoxicity data to predicted in vivo liver toxicity of lasiocarpine and riddelliine in rat

Chapter 5 Use of an in vitro-in silico testing strategy to predict inter-species and inter-ethnic human differences in liver toxicity of the pyrrolizidine alkaloids lasiocarpine and riddelliine

Chapter 6 Prediction of in vivo genotoxicity of lasiocarpine and riddelliine in rat liver using a combined in vitro-physiologically based kinetic modellingfacilitated reverse dosimetry approach

Chapter 7 General discussion

\section{Appendices}

Summary of main findings

Acknowledgment

Curriculum Vitae

List of publications

Overview of completed training activities 

Chapter 1

General introduction 



\section{The topic and aim of the thesis}

Botanicals and botanical preparations such as herbal teas, herbal medicines and plant food supplements (PFS) are widely marketed all over the world. The consumption of botanical products is increasingly high. Meantime, the reported cases of adverse events upon consumption of botanicals and botanical preparations are also rising globally (Rocha et al., 2016). To adequately guarantee the safe use of botanical preparations, it is essential to internationally harmonize the approach and procedures for the regulatory safety assessment of those products. In reality, however, the legislation and the risk assessment criteria for botanical supplements vary among different countries (Low et al., 2017). While some countries classify PFS as a food or health food (CFDA, 2016; JETRO, 2011), others regulate PFS as medicines (Health Canada 2016; TGA, 2013). To date, EU, Australia and the US have established criteria for the safety evaluation of PFS ingredients (EFSA, 2009) whereas there appears to be no such criteria in other countries.

Botanicals and botanical preparations may contain natural toxins that are of concern for human health. One group of such natural toxic compounds that raise a concern is the group of pyrrolizidine alkaloids (PAs). PAs are produced by a large number of plants, serving as part of their defence system in response to insects (Hartmann, 1999). To date, more than 660 types of PAs and PA N-oxides have been identified (Bodi et al., 2014). Previous studies have shown that herbal teas and PFS frequently contain PAs (BfR, 2013; Bodi et al., 2014; EFSA, 2016; Mulder et al., 2018; Mulder et al., 2015). Especially 1,2-unsaturated PAs are hepatotoxic and may act as genotoxic carcinogens in humans (EFSA, 2017). Human intoxication by PAs is documented in a series of case reports and related to consumption of PA-containing herbal teas and PFS, while some outbreaks even resulted in deaths related to consumption of grain contaminated with PA-producing plants (EFSA, 2017). In 2009, EFSA published guidelines for the risk assessment of botanicals and botanical preparations, defining a 2-step tiered approach for such a risk assessment. The first level can be based on available knowledge including the botanical ingredients history of use. If the available data support the conclusion that a known long term exposure to the botanical ingredient under consideration has not resulted in adverse effects, the presumption of safety could be applied. However, if such data are lacking, for the second level of the risk assessment toxicological studies are required. However, in spite of these suggested guidelines, at the current state-of-the-art risk assessment of botanical and botanical preparations is generally not required before they can enter the market (Low et al., 2017; van den Berg et al., 2014).

The aim of this thesis was to contribute to the risk assessment of botanical products containing PAs. Considering that risk management can only be based on adequate safety evaluations, the risk assessment of intake of PAs from herbal teas, herbal medicines and PFS following realistic exposure scenarios was assessed. The currently preferred approach used to assess the risk for compounds that are both genotoxic and carcinogenic is the so-called margin of exposure (MOE) approach. The MOE values can be calculated by dividing the benchmark dose level associated with $10 \%$ extra tumour 
incidence $\left(\mathrm{BMDL}_{10}\right)$ by the estimated daily intakes (EDI) (EFSA, 2005). However, in spite of the large number of PAs to which the consumer may be exposed, toxicological data on liver toxicity and carcinogenicity are available for only a limited number of PAs. Among the 1,2- unsaturated PAs, only lasiocarpine and riddelliine have been tested in a two year rodent carcinogenicity bioassay by the National Toxicology Program (NTP), and were shown to cause liver tumours (NTP, 1978, 2003). Regarding hepatotoxicity, data are only available for lasiocarpine (Dalefield et al., 2016; Jago, 1970; Nolan et al., 1966). Given the large number of PAs and the limited number of PAs for which actual in vivo toxicity and carcinogenicity data are available, the ultimate aim of the present thesis was to contribute to the risk assessment of botanical products containing PAs by applying suitable animal free testing strategies. To this end, an alternative method was developed to predict points of departure (PoDs) integrating in vitro cytotoxicity or genotoxicity assays with in silico physiologically based kinetic (PBK) modelling-facilitated reverse dosimetry. This approach may facilitate read-across from the few PAs for which toxicological data are available towards the large number of PAs for which in vivo toxicity data are lacking. In the next sections background information on PAs, including their physico-chemical characteristics, exposure characteristics, metabolism, genotoxicity, carcinogenicity, hepatotoxicity, risk assessment, and on PBK modelling-based reverse dosimetry is provided.

\section{Physico-chemical properties of the model PAs used in the present thesis}

Amongst the various PAs, especially the 1,2-unsaturated PAs are of concern because they are hepatotoxic, genotoxic and carcinogenic (EFSA, 2017). These PAs are esters of unsaturated necines having a 1,2-double bond (Figure 1). This thesis focused on two 1,2-unsaturated PAs, namely lasiocarpine and riddelliine. While lasiocarpine is a heliotridine-type PA, riddelliine is retronecinetype PA. In terms of the basic structure, lasiocarpine and riddelliine have a common bicyclic necine base with different stereochemistry at the $\mathrm{C} 7$ position; riddelliine possesses an $\mathrm{R}$ absolute configuration, lasiocarpine has an S stereochemistry (Figure 1). Moreover, lasiocarpine is an open diester and riddelliine a cyclic di-ester. The physico-chemical properties of these two PAs are also different. Lasiocarpine appears as a colourless plate or beige crystalline solid. It is soluble in most non-polar organic solvents and ethanol, but sparingly soluble in water $(0.68 \%)$ and light petroleum (NTP, 1978). Riddelliine appears as a colourless or off-white crystalline solid, it is soluble in chloroform, acetone and ethanol, and sparingly soluble in water (Chan, 1993). In spite of this, lasiocarpine and riddelliine share similar metabolic pathways, mode of action (MOA) and biological effects (Chen et al., 2010; Fu, 2016; He et al., 2017). 

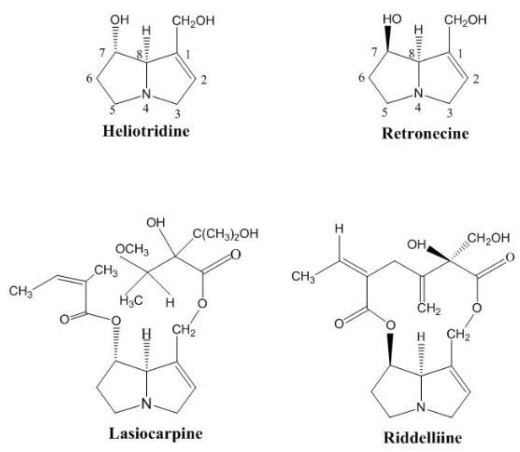

Figure 1. The schematic structure of the necine bases of PAs, and the structure of lasiocarpine and riddelliine.

\section{Exposure of humans to PAs}

Humans may be exposed to PAs through herbal teas, honey, PFS and herbal medicines (BfR, 2013; Bodi et al., 2014; EFSA, 2016; Mulder et al., 2018; Mulder et al., 2015). Recently, EFSA published a dietary exposure assessment for PAs based on the use of herbal teas, honey, herbal infusions (herbs) and PFS, reporting estimates for chronic and acute exposure to PAs via those products (EFSA, 2016). In the European population, herbal teas and herbal infusions are thought to be the major contributors to the total exposure to PAs. In order to cover the whole range of concentrations of PAs in herbal teas and herbal infusions, EFSA considered two scenarios for the estimation of dietary exposure to PAs. In the first scenario, the data of herbal tea and herbal infusion samples were obtained from national authorities according to the EFSA Article 36 grant. In the adult population ('Adults', 'Elderly', 'Very elderly'), the highest estimates of mean chronic dietary exposure to PAs ranged from the lower bound value of $31.3 \mathrm{ng} / \mathrm{kg}$ bw/day to the upper bound value of $41.8 \mathrm{ng} / \mathrm{kg}$ bw/day (LB-UB), while the maximum $95^{\text {th }}$ percentile exposure to PAs for these populations ranged from 87.7-127.2 $\mathrm{ng} / \mathrm{kg}$ bw/day (LB-UB). In a second scenario, the exposure assessment was based on samples that were collected by Tea $\&$ Herbal Infusions Europe (THIE). The highest estimates of mean chronic dietary exposure to PAs based on these input data ranged from 5.7 to $33.4 \mathrm{ng} / \mathrm{kg}$ bw/day in the adult population, while the $95^{\text {th }}$ percentile exposure estimates amounted to $15.9-78.8 \mathrm{ng} / \mathrm{kg}$ bw/day (LB-UB). When estimating the acute dietary exposure to PAs, it was assumed that all food categories would contain the highest contamination level of PAs. The estimated acute exposure levels ranged from 6 to $170 \mathrm{ng} / \mathrm{kg} \mathrm{bw} / \mathrm{day}$ for the average exposure in the adult population, and were 22 to $500 \mathrm{ng} / \mathrm{kg}$ bw/day for the $95^{\text {th }}$ percentile exposure levels (EFSA, 2016). It was also reported that the estimates of acute exposure to PAs via consumption of PFS vary considerably, depending on the type of PFS. Several PFS appeared to include PA-producing plants, although also PFS prepared from non-PA producing plants appeared to contain PAs. Consumption of such PFS dosed in liquid form was estimated to result in exposure up to $890 \mathrm{ng} / \mathrm{kg}$ bw/day of PAs in the adult population, whereas consumption of PFS in the form of 
tablets or capsules could lead to exposure up to 800 or $1800 \mu \mathrm{g} / \mathrm{kg}$ bw/day (EFSA, 2017). The estimated long-term exposure via consumption of PFS has only been quantified for pollen-based supplements and ranged from 0.7 to $11.5 \mathrm{ng} / \mathrm{kg}$ bw/day (EFSA, 2016, 2017). For the other types of PFS, such chronic PA exposure estimates have not been provided so far.

\section{Metabolic fate of PAs}

Metabolic activation is required for the biological effects of PAs. Hepatic cytochrome P450 (CYP) 2B and CYP 3A are the main enzymes to metabolize PAs (Fu et al., 2004; Huan et al., 1998; Lin et al., 2003; Prakash et al., 1999; Reed et al., 1992). After absorption in the intestine, PAs are transported into the liver where some PAs are cleaved into necines and necic acids by nonspecific esterases. These necines and necic acids are not toxic, and necines can be conjugated and excreted through the kidney and urine, representing an important pathway of PA detoxification (Chen et al., 2010; Roeder, 2000). Rats have a low esterase activity and therefore are very susceptible to the PAs (Dueker et al., 1992). Heliotridine-type PAs like lasiocarpine and retronecine-type PAs like riddelliine can be transformed to PA N-oxides through N-oxidation. These PA N-oxides can be further conjugated with polar groups and excreted from the body (Williams et al., 1989a; Williams et al., 1989b). Alternatively, the PA Noxides can be converted back into their parent PAs (Chou et al., 2003b; Wang et al., 2005c; Yan et al., 2008). In an alternative pathway of heliotridine- and retronecine-types PA metabolism, the necine bases of these PAs are hydroxylated at the $\mathrm{C} 3$ and $\mathrm{C} 8$ position forming pyrrolic ester metabolites. Pyrrolic esters are highly reactive, they may covalently react with DNA and proteins to generate DNA adducts, protein adducts and DNA and protein cross-link. In addition, pyrrolic esters can be hydrolysed into DHP (( \pm )-6,7-dihydro-7-hydroxy-1-hydroxymethyl-5H-pyrrolizine), DHP can also covalently bind with cellular DNA and proteins to produce DNA-DNA cross-link, DNA-protein crosslink, and DHP-DNA adducts (Chou et al., 2004; Fu et al., 2001; Fu et al., 2010; Fu et al., 2004; Prakash et al., 1999; Wang et al., 2005a; Wang et al., 2005b; Xia et al., 2006; Xia et al., 2013) (Figure 2). The pyrrolic esters and DHP can react with glutathione to form 7-GS-DHP and 7,9-di-GS-DHP. The 7-GS-DHP can also react with DNA to from DNA adducts (Fu et al. 2004; Xia et al., 2015, 2018). The DNA adducts are biologically active and can induce liver tumour initiation, while the protein adducts may play a role in the liver toxicity of PAs (Chen et al., 2010). The hepatic metabolism of PAs to pyrrolic esters and/or DHP is considered the bioactivation pathway (Fu et al., 2004; Prakash et al., 1999). 


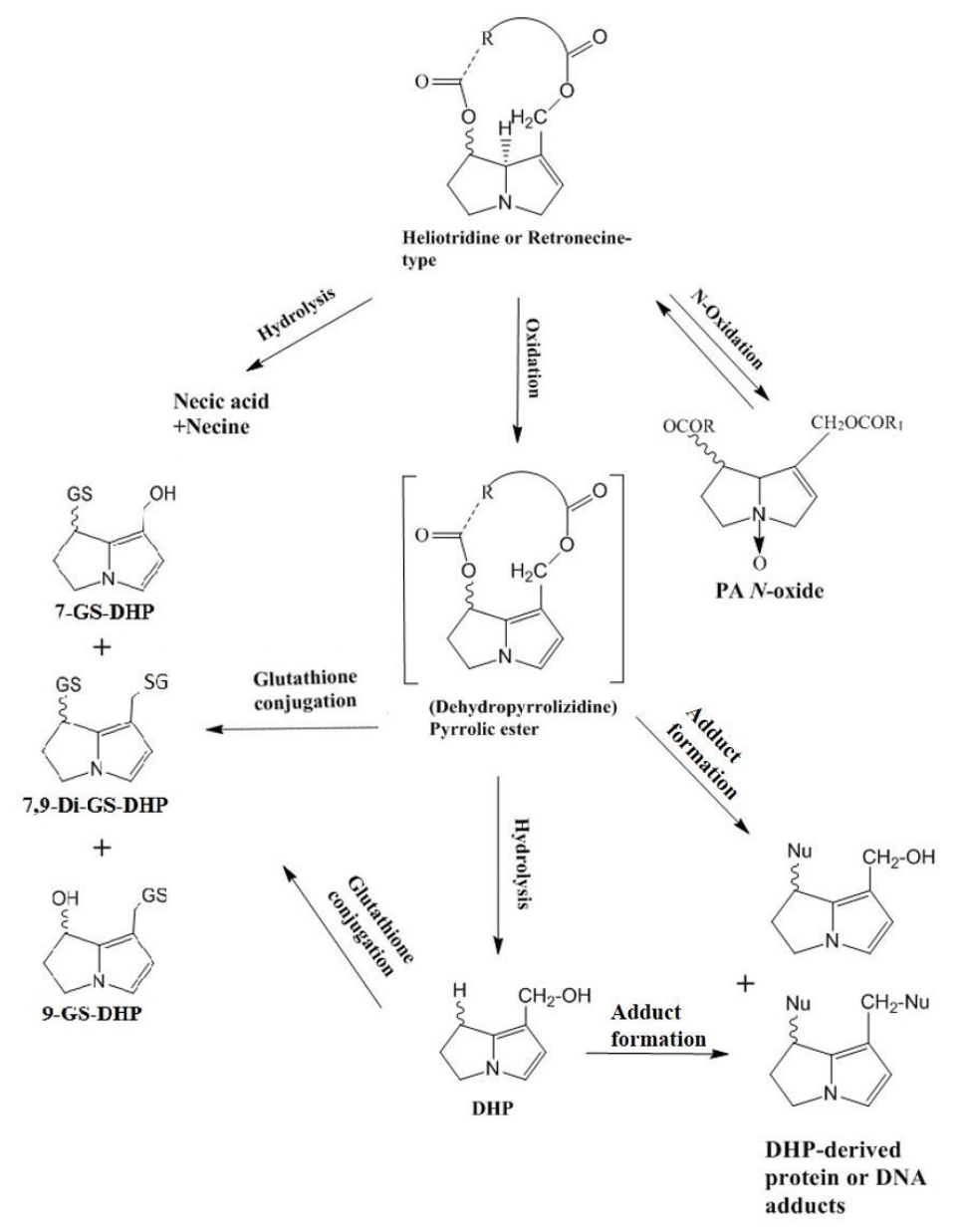

Figure 2. Heliotridine or retronecine-type PAs metabolism (Chen et al., 2010; Yang et al., 2016; Xia et al., 2015; 2018).

\section{PA genotoxicity and carcinogenicity}

PAs have been shown to induce a variety of genotoxic effects, including DNA adduct formation, DNA cross-linking, DNA strand breaks, micronuclei and chromosomal aberrations in vivo and in vitro (Allemang et al., 2018; Chen et al., 2010; NTP, 2003; Kim et al., 1995; Xia et al., 2003, 2006; Uhl et al., 2000). It was shown that all studied 1,2-unsaturated PAs including riddelliine, retrorsine, lasiocarpine, monocrotaline, riddelliine N-oxide, senkirkine, heliotrine, clivorine, and lycopsamine produced the same 4 types of DHP-derived DNA adducts in vitro and in vivo (Figure 3), and that the levels of the DHP-derived DNA adducts correlated closely with tumorigenic potency in rodents fed different doses of these PAs (Chou and Fu, 2006; Chou et al., 2004; Wang et al., 2005a; Wang et al., 2005b; Xia et al., 2006; Xia et al., 2003; Xia et al., 2004; Xia et al., 2008; Xia et al., 2013; Yang et al., 2001). 
Studies on the DNA-protein cross-linking activity were conducted using cells or isolated nuclei for the pyrrolic PA metabolites, dehydrosenecionine, dehydromonocrotaline, dehydroseneciphylline and dehydroriddelliine and revealed that all induced DNA-protein crosslinks, and the DNA-protein crosslinking potency coincided with their known toxicity potency in animal studies (Hincks et al., 1991; Kim et al., 1995, 1999). Based on these observations it was concluded that the DNA-protein cross-linking may be involved in PA-related toxicity (Coulombe Jr et al., 1999; Kim et al., 1995; Petry et al., 1984). In addition, induction of micronuclei was assessed in vitro in HepaRG cells for a series of PAs, showing dose-dependent increases in micronuclei (Allemang et al., 2018). Isatidine and monocrotaline have been shown to cause DNA strand breaks in human hepatoma cells (HepG2 cells) and human glioblastoma cells (Glial cells) using the comet assay, with the treatment causing significant dose-dependent increases in cell DNA breaks (Silva-Neto et al., 2010; Uhl et al., 2000). These genotoxic effects may induce gene mutations and have been associated with the induction of cancer cells and tumour formation (Chen et al., 2010; Hoeijmakers, 2009). Oral administration of lasiocarpine as well as riddelliine for 2 years to rats has been shown to induce liver haemangiosarcomas (NTP, 1978, 2003). For lasiocarpine, liver haemangiosarcomas were found at frequencies amounting to $5 / 24,11 / 23$ and $13 / 23$ at dose levels of $0.35,0.75$ and $1.5 \mathrm{mg} / \mathrm{kg}$ bw/day (NTP, 1978). For riddelliine, liver haemangiosarcomas were found at frequencies amounting to $3 / 50$ and $38 / 50$ at dose levels of 0.236 and $0.714 \mathrm{mg} / \mathrm{kg}$ bw/day (NTP, 2003). These results demonstrate the carcinogenicity of lasiocarpine and riddelliine upon chronic oral exposure. Some other PAs, including monocrotaline, clivorine, senkirkine, and symphytine, have also been shown to cause liver tumours in rodents, albeit upon different routes of exposure (Hirono et al., 1979; Hirono et al., 1976; Kuhara et al., 1980; Shumaker et al., 1976). Subcutaneous (s.c.) administration of $0.08 \mathrm{mg} / \mathrm{kg}$ bw/day monocrotaline to male rats resulted in an increase in a number of tumours, principally liver cell carcinomas and pulmonary adenocarcinomas (Shumaker et al., 1976). Clivorine induced liver neoplastic nodules in rats when they were exposed to $1.56 \mathrm{mg} / \mathrm{kg}$ bw/day clivorine via drinking water (Kuhara et al., 1980). Senkirkine and symphytine have been shown to induce liver cell adenomas and liver haemangioendothelial sarcomas in rats upon administration via i.p. injection of $1.62 \mathrm{mg} / \mathrm{kg}$ bw/day senkirkine and $0.92 \mathrm{mg} / \mathrm{kg}$ bw/day symphytine, respectively (Hirono et al., 1979). To date, there is no direct evidence showing that PA intake can lead to liver tumours in humans. However, considering the prevalence of PAs all over the world and the evidence of PA-induced carcinogenicity in rodents, human exposure to PAs may raise a concern. The International Agency for Research on Cancer (IARC) classified lasiocarpine and riddelliine in Group 2B, defined as being possibly carcinogenic to humans (IARC, 1976, 2002). 


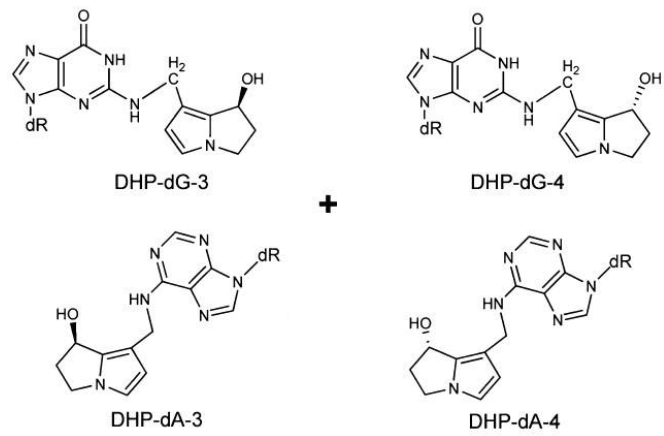

Figure 3. The 4 types of DHP-derived DNA adducts (Xia et al., 2013).

\section{PAs hepatotoxicity}

Compared to the lack of data related to the potential carcinogenicity of PAs in humans, PA induced acute hepatotoxicity has been reported in several human case studies. This acute liver toxicity was generally characterized by several liver diseases, including hemorrhagic necrosis, hepatomegaly, ascites. Cases of sub-acute exposure have been reported to result in a blockage of hepatic veins, leading to hepatic veno-occlusive disease (HVOD) (EFSA, 2011, 2017). Human poisonings caused by intake of PA-containing plants have been reported in several countries (Mohabbat et al., 1976; Prakash et al., 1999; Roulet et al., 1988; Sperl et al., 1995; Tandon et al., 1976). In 1989, the International Program on Chemical Safety (IPCS) concluded that consumption of contaminated grain or the use of PA-containing plants as herbal medicine, beverages, or food by man, or grazing on contaminated pastures by animals, may cause acute or chronic toxicity (IPCS, 1988). So far, an acute reference dose (ARfD) for PAs has not been established, while a lowest known dose of approximately $2 \mathrm{mg} / \mathrm{kg}$ bw per day associated with acute/short-term effects has been identified based on two human poisoning cases. One of the human cases was a 6-month-old girl who was exposed to a daily dose of approximately $0.8-1.7 \mathrm{mg} \mathrm{PA} / \mathrm{kg}$ bw for 2 weeks and developed HVOD. Another case was a 2-monthold boy who received an approximate dose of $3 \mathrm{mg} / \mathrm{kg}$ bw for 4 days, with a fatal outcome (EFSA, 2017). With respect to PA induced hepatotoxicity in rodents, data on acute liver toxicity are only available for lasiocarpine (Dalefield et al., 2016; Jago, 1970; Nolan et al., 1966). Single oral administration $0-80 \mathrm{mg} / \mathrm{kg}$ bw/day lasiocarpine to rats resulted in acute zonal hepatic necrosis, megalocytosis and subacute or chronic hepatitis (Nolan et al., 1966). Through i.p. injection of 0, 10.28, $20.56,41.1,82.2,164.4 \mathrm{mg} / \mathrm{kg}$ bw/day lasiocarpine to rats, the dose-dependent increased incidence of slight periportal nuclear enlargement, periportal megalocytosis, megalocytosis and necrosis has been identified (Jago, 1970). Lasiocarpine also has been found to induced alanine amino transferase activity when rats were exposed to $0.6,1.2,2.5 \mathrm{mg} / \mathrm{kg}$ bw/day lasiocarpine for over 28 days (Dalefield et al., 2016). 


\section{Risk assessment of PAs}

PAs are considered genotoxic carcinogens. Data of genotoxic carcinogens in human epidemiological studies are rarely available, probably due to the fact that such data are difficult to obtain from the human population because effects may be masked by many confounding factors and their detection may require extremely large study populations. Thus, to assess the risk of genotoxic carcinogens to human health, often data based on animal studies are used. There is no doubt that differences in biological responses to genotoxic carcinogens exist between experimental animals and humans, meaning that data resulting from animal studies cannot be regarded as fully representative for humans. Therefore, uncertainty connected to the extrapolation of animal data to the human situation should be considered when assessing the risk of genotoxic carcinogens to human health based on data derived from animal experiments. One of the suitable methods for preforming such a risk assessment is the MOE approach (EFSA, 2005). The MOE is the dimensionless ratio between a reference point based on epidemiologic or experimental data on tumour incidence and the EDI in the relevant human population (Barlow et al., 2006; EFSA, 2005). To calculate the reference point, a preferred method is the BenchMark Dose (BMD) approach. The BMD can be obtained without extrapolation by fitting the mathematical models to the available data (Barlow et al., 2006). The BMDL, defined as the one-sided $95 \%$ lower confidence limit of the BMD, takes statistic uncertainties into account and is thought to be the preferred reference point for calculating the MOE (Barlow et al., 2006). BMD and BMDL values can be calculated for a pre-defined increased tumour incidence compared to the background, that is the BenchMark Response (BMR) including 1\%, 5\% or 10\% extra incidence of tumours (EPA, 1995). In general, using the $\mathrm{BMDL}_{10}$ is preferred for calculating the MOE since the use of lower incidences will increase the uncertainty as $1 \%$ or $5 \%$ may be outside the experimentally observed incidences (Barlow et al., 2006). To obtain a $\mathrm{BMDL}_{10}$ value for a specific substance, generally a long term animal carcinogenicity study using either rats or mice is required, which has to meet three requirements including: administration of three different experimental doses, sufficient statistical power requiring at least 50 animals per treatment and accomplishment of a two-year experimental period (Edler et al., 2014). Some studies have experimental durations less than the required span of a two-year (104 weeks) period, in which the actual period of the treatment can be even shorter. In this case the dose levels should be adjusted according to the following formula: dose $\times($ weeks of treatment $/ 104) \times($ weeks of observation/104) (ECHA, 2008). The adjusted dose levels are then used to derive the $\mathrm{BMDL}_{10}$ value. In case that the data cannot meet the requirement for calculation of a $\mathrm{BMDL}_{10}$, EFSA has recommended use of the T25 or T10 (the dose level causing $25 \%$ or $10 \%$ extra tumour incidence) as an alternative point of departure (PoD) for MOE calculation. T25 values are calculated by dividing the extra risk (\% incidence in the dose group minus \% incidence in the control group) by the non-affected fraction in the control population (100 minus background response in \%), and converting the dose level causing this tumour incidence to the dose level causing $25 \%$ extra tumour incidence by linear 
extrapolation. T10 values are obtained by multiplying T25 values by 10/25. At the current state of the art, the T25 with a MOE cut-off value of 25000 and the T10 with a MOE cut-off value of 10000 (also applied when using the $\mathrm{BMDL}_{10}$ ) are used when a $\mathrm{BMDL}_{10}$ is not available. A value of 10000 incorporates three uncertainty factors including a factor 100 for species differences and human variability in kinetics and dynamics, a factor of 10 for the variability in cell cycle control and DNA repair within humans, and a factor of 10 because the $\mathrm{BMDL}_{10}$ is not a no observed adverse effect level (NOAEL) (EFSA, 2005).

The MOE is based on the assumption of daily life-time exposure. Thus, the risk assessments for exposure to PAs via herbal teas, herbal medicines and PFS that have been done previously were also based on the assumption of daily life-time exposure to these products (BfR, 2013; Chen et al., 2017; EFSA, 2016, 2017). Such an assumption may not be true as people might rather consume these products occasionally or only during certain periods of, for example, illness. At the current state-ofthe-art there is no generally accepted method to correct for this shorter than life-time daily exposure. Nevertheless, application of the MOE approach combined with Haber's rule for risk assessment taking into account shorter than life-time realistic exposure scenarios could be considered. According to Haber's rule, the toxic effects are correlated linearly with the timespan of exposure and the concentration or dose of a toxic compound (i.e. $\mathrm{C} \times \mathrm{T}=\mathrm{k}$, where $\mathrm{C}$ is concentration or dose, $\mathrm{T}$ is time of exposure, and $\mathrm{k}$ is a constant toxic response for the specific substance) (Doull and Rozman, 2000). Employing Haber's rule and assuming a lifetime expectancy of 75 years (Felter et al., 2011; van den Berg et al., 2014), the EDI values for for example 2 weeks yearly exposure during a lifetime will be 52 weeks per year $/ 2$ weeks $=26$ times lower, resulting in 26 times higher MOE values.

A BMDL 10 value is required for the MOE approach. So far, amongst all PAs only for lasiocarpine and riddelliine 2-year animal studies are available that provided data suitable to derive $\mathrm{BMDL}_{10}$ values. The $\mathrm{BMDL}_{10}$ of $0.07 \mathrm{mg} / \mathrm{kg}$ bw/day for lasiocarpine was used in first instance as the PoD value to calculate MOEs for exposure to PAs (EFSA, 2011). Recently EFSA defined a reference point of 0.237 $\mathrm{mg} / \mathrm{kg}$ /day for riddelliine as the PoD to assess the carcinogenicity risk of PAs based on an updated BMD modelling approach. This was done because it was considered that the $\mathrm{BMDL}_{10}$ obtained by modelling of the data on liver tumour formation by lasiocarpine were affected by a high degree of uncertainty (EFSA, 2017). The EDI values were calculated based on total PA levels in herbal products, taking into consideration the combined exposure to different PAs (BfR, 2013; EFSA, 2016, 2017). When considering combined exposure, both equal toxic potency or different potency of the different PAs could be considered. This implies that the response to the mixtures can be calculated by summing up the EDIs of the PAs assuming equal potency, or using their relative potency (REP) to 'adjust' the individual PA concentrations. Interim REP factors for PAs were proposed by Merz and Schrenk (2016), based on available literature data on in vitro cytotoxicity, genotoxicity in Drosophila, and acute toxicity in rodents (LD50), and REP factors were defined for the most relevant structural types 
of PAs (Merz and Schrenk, 2016). It should be noticed that these interim REP values did not take the relative potency of PAs for tumour formation into account, because the lack of carcinogenicity studies for PAs hampers such a more detailed (combined) risk assessment.

\section{PBK modelling-based reverse dosimetry}

Due to the lack of liver toxicity and carcinogenicity data for most PAs, and also considering economic and ethical constraints that limit the possibilities to perform toxicity studies for all relevant PAs, alternative testing strategies for estimating the toxicity of different PAs are required. However, use of alternative testing methods instead of animal studies in quantitative risk assessment, requires translation of in vitro concentration-response curves to in vivo dose-response curves. This can be achieved using physiologically based kinetic (PBK) modelling-based reverse dosimetry. It has been shown that data on in vivo toxicity can be adequately predicted by translation of in vitro concentration-response curves to in vivo dose-response curves for toxicity using this in vitro-in silico approach (Abdullah et al., 2016; Li et al., 2017; Louisse et al., 2015; Louisse et al., 2010; Strikwold et al., 2017; Zhang et al., 2018). In this way in vivo dose effect levels and PoDs have been defined, for example for developmental toxicity (Li et al., 2017; Louisse et al., 2015; Louisse et al., 2010; Strikwold et al., 2017), kidney toxicity (Abdullah et al., 2016), and estrogenicity (Zhang et al., 2018). The present thesis applied the approach for liver toxicity and genotoxicity of lasiocarpine and riddelliine, the two PAs for which rodent data on liver toxicity and/or carcinogenicity are available. PBK models require different parameters including physiological, physico-chemical and metabolic parameters to mathematically describe the absorption, distribution, metabolism and excretion (ADME) of the compound of interest in the relevant organ and species (Chiu et al., 2007; Krewski et al., 1994; Rietjens et al., 2011). PBK modelling can take time- and dose-dependent changes in metabolism into account, and blood and tissue concentrations of a compound and its relevant metabolites can be predicted over time (Rietjens et al., 2011). The use of PBK modelling-based reverse dosimetry to predict in vivo dose-response curves and PoDs for risk assessment involves several steps: (1) establishment of an in vitro concentration-response curve for a selected relevant endpoint using an adequate in vitro assay model, (2) development of the PBK model describing in vivo kinetics of the parent compound and/or metabolites of interest in the relevant tissue, (3) evaluation of the developed PBK model against available literature data on kinetics, (4) translation of the in vitro concentrationresponse curves from the in vitro assay into in vivo dose-response curves using PBK modelling-based reversed dosimetry taking differences in protein binding of the compound of interest in the in vitro model and the in vivo situation into account, (5) BMD analysis on the predicted in vivo dose-response curve to obtain a PoD, (6) evaluation of the predicted in vivo dose response curves and/or the predicted PoD against available literature data. Development of a PBK model means defining the structure of the model including organs and tissues that are considered relevant for the kinetic processes and the toxicity of the compound of interest. A PBK model generally includes separate 
compartments for these tissues relevant for the kinetics and toxicity of the compound, while the remaining non-target tissue compartments can be pooled in compartments representing slowly or richly perfused tissue. All the compartments are mutually connected through the system circulation (Figure. 4). For each compartment, mathematical equations are defined to describe the kinetic processes of the compound. For these equations, the relevant physiological, anatomical, physicochemical and kinetic values need to be defined. These parameters can be obtained from literature, and from in vivo or in vitro assays. Once the mathematic model is established and the relevant parameters have been defined, the equations can be solved using specific software in order to predict the maximum concentration $\left(\mathrm{C}_{\max }\right)$ or the area under the concentration curve (AUC) of the compound in plasma or in any tissue of interest. In a next step each concentration (or AUC) that is used in the in vitro assay is set equal to $\mathrm{C}_{\max }$ (or $\mathrm{AUC}$ ) in the blood or in a specific tissue, after correction for possible differences in protein binding due to different protein levels in the medium of the in vitro assay and the in vivo tissue. Then this $\mathrm{C}_{\max }$ (or AUC) is used as input in the PBK model to calculate the oral dose that results in this concentration (or AUC). The AUC values are calculated by multiplying the used concentration with the assay time (Daston et al., 2010). The $C_{\max }$ (or AUC)response curves can be translated to the in vivo situation by assessing which oral doses are required to reach these $\mathrm{C}_{\max }$ (or AUC) levels in the blood or specific tissue. Use of $\mathrm{C}_{\max }$ or AUC depends on the toxicological endpoint studied, with acute toxicity being better reflected by $\mathrm{C}_{\max }$ values while longer term toxicity for which the effects accumulate over the time of exposure, such as for example genotoxicity or carcinogenicity, might be better predicted using the AUC. Upon translation of the in vitro data to an in vivo dose response curve, the predicted dose-response curves and PoDs derived from them, can be evaluated by comparison to dose-response curves reported in the literature and/or to PoDs derived from these available in vivo data. In addition, a sensitivity analysis can be applied to evaluate how the input parameters affect the output of the model.

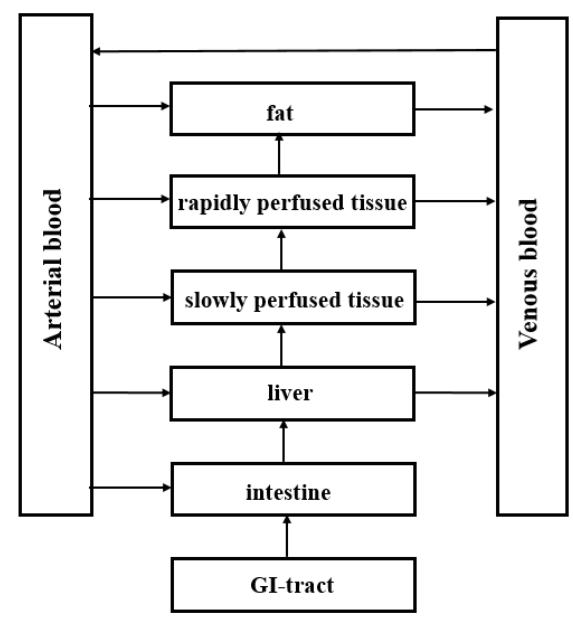

Figure. 4 Schematic diagram of the generic PBK model for lasiocarpine and riddelliine in rat and human. 


\section{Objectives and outline of the thesis}

The aim of this thesis was to contribute to the risk assessment for PAs in botanical and botanical preparations including herbal teas, herbal medicines and PFS based on life-time exposure and realistic exposure scenarios by developing and applying suitable alternative testing strategies. To this end the thesis also aimed to investigate whether PBK modelling-based reverse dosimetry could adequately convert in vitro concentration-response curves to in vivo dose-response curves to predict liver toxicity and genotoxicity induced by lasiocarpine and riddelliine.

Chapter 1, the introduction chapter, starts with a short background and definition of the aim of the present thesis, followed by a description of the physico-chemical properties, exposure characteristics, metabolism, genotoxicity, carcinogenicity, hepatotoxicity, and risk assessment of PAs, and of PBK modelling-based reverse dosimetry.

Chapter 2 presents an overview of tumour data available for PAs including, in addition to lasiocarpine and riddelliine, monocrotaline, clivorine, senkirkine and symphytine and PoDs derived from them. A risk assessment for (combined) exposure to PAs via herbal teas and PFS was performed, based on the MOE approach using the $\mathrm{BMDL}_{10}$ for lasiocarpine and mean PA levels corrected by REP factors.

Chapter 3 evaluates the risk of exposure to PAs through consumption of herbal teas, herbal medicines and PFS following realistic exposure scenarios. To this end herbal teas were used to quantify the effect of comminution on the levels of PAs extracted into hot water and resulting PA exposure as compared to use of the intact leaves. The MOE approach was used to evaluate the consequences of this difference for the associated risks. Furthermore, considering medicinal use of these botanical preparations, the risk under shorter than life-time more realistic exposure scenarios was also evaluated.

Chapter 4 describes the development and evaluation of PBK models for lasiocarpine and riddelliine in rat, and use of these models to predict the PoDs for liver toxicity of lasiocarpine and riddelliine. To this end in vitro concentration-response curves for cytotoxicity in primary rat hepatocytes were converted to in vivo dose-response curves for liver toxicity in rat using PBK modelling-based reverse dosimetry. The model performance was evaluated by comparison of the predicted PoDs to in vivo available liver toxicity data.

Chapter 5 extends the PBK model-based reverse dosimetry approach by defining PBK models for humans and predicting inter-species and inter-ethnic human differences in liver toxicity of lasiocarpine and riddelliine. The concentration-response curves of in vitro cytotoxicity of lasiocarpine and riddelliine defined in pooled primary human hepatocytes were translated to in vivo dose-response curves by PBK models developed using kinetic data obtained from incubations with pooled tissue 
fractions from Chinese and Caucasian individuals, providing PBK models for the average Chinese and average Caucasian, respectively.

Chapter 6 investigates whether the developed PBK modelling-facilitated reverse dosimetry can adequately translate in vitro concentration-response curves for genotoxicity of lasiocarpine and riddelliine, to in vivo dose-response curves for genotoxicity induced by lasiocarpine and riddelliine in rat. To achieve this aim, the in vitro concentration-responses curves obtained from in vitro genotoxicity studies with primary rat hepatocytes using the $\gamma \mathrm{H} 2 \mathrm{AX}$ assay were translated into in vivo dose-response curves by using PBK modelling-facilitated reverse dosimetry. The predicted PoDs for rat were compared to available in vivo rodent data on genotoxicity and carcinogenicity induced by lasiocarpine and riddelliine.

Chapter 7 summarizes the results obtained in the thesis, provides an overall discussion and presents the future perspectives that follow from the results obtained. 


\section{References}

Abdullah, R., Alhusainy, W., Woutersen, J., Rietjens, I.M., Punt, A., 2016. Predicting points of departure for risk assessment based on in vitro cytotoxicity data and physiologically based kinetic (PBK) modeling: the case of kidney toxicity induced by aristolochic acid I. Food and Chemical Toxicology 92, 104-116.

Allemang, A., Mahony, C., Lester, C., Pfuhler, S., 2018. Relative potency of fifteen pyrrolizidine alkaloids to induce DNA damage as measured by micronucleus induction in HepaRG human liver cells. Food and chemical toxicology 121, 72-81.

Barlow, S., Renwick, A., Kleiner, J., Bridges, J., Busk, L., Dybing, E., Edler, L., Eisenbrand, G., FinkGremmels, J., Knaap, A., 2006. Risk assessment of substances that are both genotoxic and carcinogenic: report of an International Conference organized by EFSA and WHO with support of ILSI Europe. Food and chemical toxicology 44, 1636-1650.

BfR, 2013. Pyrrolizidine alkaloids in herbal teas and teas. BfR Opinion No. 018/2013 of 5 July 2013. Pyrrolizidine alkaloids-in-herbal-teas-and-teas.

Bodi, D., Ronczka, S., Gottschalk, C., Behr, N., Skibba, A., Wagner, M., Lahrssen-Wiederholt, M., Preiss-Weigert, A., These, A., 2014. Determination of pyrrolizidine alkaloids in tea, herbal drugs and honey. Food Additives \& Contaminants: Part A 31, 1886-1895.

CFDA, 2016. "Health food registration and record management approach" (State Food and Drug Administration Order No. 22)

Chan, P., 1993. NTP technical report on the toxicity studies of Riddelliine (CAS No. 23246-96-0) Administered by Gavage to F344 Rats and B6C3F1 Mice. Toxicity report series 27, 1-D9.

Chen, L., Mulder, P.P., Louisse, J., Peijnenburg, A., Wesseling, S., Rietjens, I.M., 2017. Risk assessment for pyrrolizidine alkaloids detected in (herbal) teas and plant food supplements. Regulatory Toxicology and Pharmacology 86, 292-302.

Chen, T., Mei, N., Fu, P.P., 2010. Genotoxicity of pyrrolizidine alkaloids. Journal of Applied Toxicology: An International Journal 30, 183-196.

Chiu, W.A., Barton, H.A., DeWoskin, R.S., Schlosser, P., Thompson, C.M., Sonawane, B., Lipscomb, J.C., Krishnan, K., 2007. Evaluation of physiologically based pharmacokinetic models for use in risk assessment. Journal of Applied Toxicology: An International Journal 27, 218-237.

Chou, M.W., Fu, P.P., 2006. Formation of DHP-derived DNA adducts in vivo from dietary supplements and Chinese herbal plant extracts containing carcinogenic pyrrolizidine alkaloids. Toxicology and industrial health 22, 321-327.

Chou, M.W., Wang, Y.-P., Yan, J., Yang, Y.-C., Beger, R.D., Williams, L.D., Doerge, D.R., Fu, P.P., 2003b. Riddelliine N-oxide is a phytochemical and mammalian metabolite with genotoxic activity that is comparable to the parent pyrrolizidine alkaloid riddelliine. Toxicology letters $145,239-247$.

Chou, M.W., Yan, J., Nichols, J., Xia, Q., Beland, F.A., Chan, P.-C., Fu, P.P., 2004. Correlation of DNA adduct formation and riddelliine-induced liver tumorigenesis in $\mathrm{F} 344$ rats and B6C3F1 mice [Cancer Lett. 193 (2003) 119-125]. Cancer letters 207, 119-125.

Coulombe Jr, R.A., Drew, G.L., Stermitz, F.R., 1999. Pyrrolizidine alkaloids crosslink DNA with actin. Toxicology and applied pharmacology 154, 198-202.

Dalefield, R.R., Gosse, M.A., Mueller, U., 2016. A 28-day oral toxicity study of echimidine and lasiocarpine in Wistar rats. Regulatory Toxicology and Pharmacology 81, 146-154.

Daston, G.P., Chapin, R.E., Scialli, A.R., Piersma, A.H., Carney, E.W., Rogers, J.M., Friedman, J.M., 2010. A different approach to validating screening assays for developmental toxicity. Birth Defects Research Part B: Developmental and Reproductive Toxicology 89, 526-530.

Doull, J., Rozman, K., 2000. Using Haber's Law to define the margin of exposure. Toxicology 149, 1.

Dueker, S.R., Lamé, M.W., Morin, D.E.X.T.E.R., Wilson, D.W. and Segall, H.J., 1992. Guinea pig and rat hepatic microsomal metabolism of monocrotaline. Drug metabolism and disposition, 20(2), pp.275-280.

ECHA, M., 2008. Guidance on information requirements and chemical safety assessment. Chapter R 8. 
Edler, L., Hart, A., Greaves, P., Carthew, P., Coulet, M., Boobis, A., Williams, G.M., Smith, B., 2014. Selection of appropriate tumour data sets for Benchmark Dose Modelling (BMD) and derivation of a Margin of Exposure (MoE) for substances that are genotoxic and carcinogenic: Considerations of biological relevance of tumour type, data quality and uncertainty assessment. Food and chemical toxicology 70, 264-289.

EFSA, 2005. Opinion of the Scientific Committee on a request from EFSA related to a harmonised approach for risk assessment of substances which are both genotoxic and carcinogenic. EFSA Journal 3, 282.

EFSA, 2009. Guidance on safety assessment of botanicals and botanical preparations intended for use as ingredients in food supplements. EFSA Journal 7, 1249.

EFSA, 2011. Scientific opinion on pyrrolizidine alkaloids in food and feed. EFSA Journal 9, 2406.

EFSA, 2016. Dietary exposure assessment to pyrrolizidine alkaloids in the European population. EFSA Journal 14, e04572.

EFSA, 2017. Risks for human health related to the presence of pyrrolizidine alkaloids in honey, tea, herbal infusions and food supplements. EFSA Journal 15.

EPA, 1995. The use of the benchmark dose approach in health risk assessment. Risk Assessment Forum, US Environmental Protection Agency.

Felter, S.P., Conolly, R.B., Bercu, J.P., Bolger, P.M., Boobis, A.R., Bos, P.M., Carthew, P., Doerrer, N.G., Goodman, J.I., Harrouk, W.A., 2011. A proposed framework for assessing risk from lessthan-lifetime exposures to carcinogens. Critical reviews in toxicology 41, 507-544.

Fu, P., Chou, M., Xia, Q., Yang, Y.-C., Yan, J., Doerge, D., Chan, P., 2001. Genotoxic pyrrolizidine alkaloids and pyrrolizidine alkaloid N-oxides-mechanisms leading to DNA adduct formation and tumorigenicity. Journal of Environmental Science and Health, Part C 19, 353-385.

Fu, P.P., 2016. Pyrrolizidine alkaloids: metabolic activation pathways leading to liver tumor initiation. Chemical research in toxicology 30, 81-93.

Fu, P.P., Chou, M.W., Churchwell, M., Wang, Y., Zhao, Y., Xia, Q., Gamboa da Costa, G.a., Marques, M.M., Beland, F.A., Doerge, D.R., 2010. High-performance liquid chromatography electrospray ionization tandem mass spectrometry for the detection and quantitation of pyrrolizidine alkaloid-derived DNA adducts in vitro and in vivo. Chemical research in toxicology 23, 637-652.

Fu, P.P., Xia, Q., Lin, G., Chou, M.W., 2004. Pyrrolizidine alkaloids-genotoxicity, metabolism enzymes, metabolic activation, and mechanisms. Drug metabolism reviews 36, 1-55.

Health Canada, 2016. About natural health product regulations in Canada-Health Canada.

Hartmann, T., 1999. Chemical ecology of pyrrolizidine alkaloids. Planta 207, 483-495.

He, X., Xia, Q., Fu, P.P., 2017. 7-Glutathione-pyrrole and 7-cysteine-pyrrole are potential carcinogenic metabolites of pyrrolizidine alkaloids. Journal of Environmental Science and Health, Part C 35, 69-83.

Hirono, I., Haga, M., Fujii, M., Matsuura, S., Matsubara, N., Nakayama, M., Furuya, T., Hikichi, M., Takanashi, H., Uchida, E., 1979. Induction of hepatic tumors in rats by senkirkine and symphytine. Journal of the National Cancer Institute 63, 469-472.

Hirono, I., Mori, H., Culvenor, C.C., 1976. Carcinogenic activity of coltsfoot, Tussilago farfara L. GANN Japanese Journal of Cancer Research 67, 125-129.

Hoeijmakers, J.H., 2009. DNA damage, aging, and cancer. New England Journal of Medicine 361, $1475-1485$.

Huan, J.-Y., Miranda, C.L., Buhler, D.R., Cheeke, P.R., 1998. The roles of CYP3A and CYP2B isoforms in hepatic bioactivation and detoxification of the pyrrolizidine alkaloid senecionine in sheep and hamsters. Toxicology and applied pharmacology 151, 229-235.

Hincks, J.R., Kim, H.Y., Segall, H.J., Molyneux, R.J., Stermitz, F.R. and Coulombe Jr, R.A., 1991. DNA cross-linking in mammalian cells by pyrrolizidine alkaloids: structure-activity relationships. Toxicology and applied pharmacology, 111(1), pp.90-98.

IARC, 1976. IARC Monographs on Evaluation of Carcinogenic Risk of chemicals to Man: Some Naturally Occuring Substances. 10.

IARC, 2002. IARC Monographs on Evaluation of Carcinogenic Risk of Chemicals to Humans: Some Traditional Herbal Medicines, Some Mycotoxins,Naphthalene and Styrene. 82 
IPCS, 1988. International Programme on Chemical Safety Health and Safety Environmental Health Criteria 80: Pyrrolizidine alkaloids.

JETRO, 2011. Guidebook for export to Japan (Food Articles) < Health foods and dietary supplements>March.

Jago, M.V., 1970. A method for the assessment of the chronic hepatoxicity of pyrrolizidine alkaloids. Australian Journal of Experimental Biology and Medical Science 48, 93-103.

Kim, H.-Y., Stermitz, F.R., Coulombe Jr, R.A., 1995. Pyrrolizidine alkaloid-induced DNA-protein cross-links. Carcinogenesis 16, 2691-2697.

Kim, H.Y., Stermitz, F.R., Li, J.K. and Coulombe Jr, R.A., 1999. Comparative DNA cross-linking by activated pyrrolizidine alkaloids. Food and chemical toxicology, 37(6), pp.619-625.

Krewski, D., Withey, J.R., Ku, L.-f., Andersen, M.E., 1994. Applications of physiologic pharmacokinetic modeling in carcinogenic risk assessment. Environmental Health Perspectives $102,37-50$.

Kuhara, K., Takanashi, H., Hirono, I., Furuya, T., Asada, Y., 1980. Carcinogenic activity of clivorine, a pyrrolizidine alkaloid isolated from Ligularia dentata. Cancer letters 10, 117-122.

Li, H., Zhang, M., Vervoort, J., Rietjens, I.M., van Ravenzwaay, B., Louisse, J., 2017. Use of physiologically based kinetic modeling-facilitated reverse dosimetry of in vitro toxicity data for prediction of in vivo developmental toxicity of tebuconazole in rats. Toxicology letters 266 , $85-93$

Lin, G., Cui, Y.-Y., Liu, X.-Q., 2003. Gender differences in microsomal metabolic activation of hepatotoxic clivorine in rat. Chemical research in toxicology 16, 768-774.

Louisse, J., Bosgra, S., Blaauboer, B.J., Rietjens, I.M., Verwei, M., 2015. Prediction of in vivo developmental toxicity of all-trans-retinoic acid based on in vitro toxicity data and in silico physiologically based kinetic modeling. Archives of toxicology 89, 1135-1148.

Louisse, J., de Jong, E., van de Sandt, J.J., Blaauboer, B.J., Woutersen, R.A., Piersma, A.H., Rietjens, I.M., Verwei, M., 2010. The use of in vitro toxicity data and physiologically based kinetic modeling to predict dose-response curves for in vivo developmental toxicity of glycol ethers in rat and man. Toxicological Sciences 118, 470-484.

Low, T.Y., Wong, K.O., Yap, A.L., De Haan, L.H., Rietjens, I.M., 2017. The regulatory framework across international jurisdictions for risks associated with consumption of botanical food supplements. Comprehensive Reviews in Food Science and Food Safety 16, 821-834.

Merz, K.H., Schrenk, D., 2016. Interim relative potency factors for the toxicological risk assessment of pyrrolizidine alkaloids in food and herbal medicines. Toxicology letters 263, 44-57.

Mohabbat, O., Younos, M.S., Merzad, A., Srivastava, R., Sediq, G.G., Aram, G., 1976. An outbreak of hepatic veno-occlusive disease in north-western Afghanistan. The Lancet 308, 269-271.

Mulder, P.P., López, P., Castelari, M., Bodi, D., Ronczka, S., Preiss-Weigert, A., These, A., 2018. Occurrence of pyrrolizidine alkaloids in animal-and plant-derived food: results of a survey across Europe. Food Additives \& Contaminants: Part A 35, 118-133.

Mulder, P.P., Sánchez, P.L., These, A., Preiss-Weigert, A., Castellari, M., 2015. Occurrence of Pyrrolizidine Alkaloids in food. EFSA Supporting Publications 12, 859E.

Nolan, J.P., Scheig, R.L., Klatskin, G., 1966. Delayed hepatitis and cirrhosis in weanling rats following a single small dose of the senecio alkaloid, lasiocarpine. The American journal of pathology 49, 129.

NTP, 1978. Bioassay of lasiocarpine for possible carcinogenicity. National Cancer Institute carcinogenesis technical report series 39,1 .

NTP, 2003. Toxicology and carcinogenesis studies of riddelliine (CAS No. 23246-96-0) in F344/N rats and B6C3F1 mice (gavage studies). National Toxicology Program technical report series, 1.

Petry, T.W., Bowden, G.T., Huxtable, R.J., Sipes, I.G., 1984. Characterization of hepatic DNA damage induced in rats by the pyrrolizidine alkaloid monocrotaline. Cancer research 44, 15051509.

Prakash, A.S., Pereira, T.N., Reilly, P.E., Seawright, A.A., 1999. Pyrrolizidine alkaloids in human diet. Mutation Research/Genetic Toxicology and Environmental Mutagenesis 443, 53-67.

Reed, R., Miranda, C., Kedzierski, B., Henderson, M., Buhler, D., 1992. Microsomal formation of a pyrrolic alcohol glutathione conjugate of the pyrrolizidine alkaloid senecionine. Xenobiotica 22, 1321-1327. 
Rietjens, I.M., Louisse, J., Punt, A., 2011. Tutorial on physiologically based kinetic modeling in molecular nutrition and food research. Molecular nutrition \& food research 55, 941-956.

Roeder, E., 2000. Medicinal plants in China containing pyrrolizidine alkaloids. Pharmazie 55, 711-726.

Roulet, M., Laurini, R., Rivier, L., Calame, A., 1988. Hepatic veno-occlusive disease in newborn infant of a woman drinking herbal tea. Journal of Pediatrics.

Rocha, T., Amaral, J.S. and Oliveira, M.B.P., 2016. Adulteration of dietary supplements by the illegal addition of synthetic drugs: a review. Comprehensive reviews in food science and food safety, $15(1)$, pp.43-62.

Shumaker, R., Robertson, K., Hsu, I., Allen, J., 1976. Neoplastic transformation in tissues of rats exposed to monocrotaline or dehydroretronecine. Journal of the National Cancer Institute 56, 787-790.

Silva-Neto, J., Barreto, R., Pitanga, B., Souza, C., Silva, V., Silva, A., Velozo, E.d.S., Cunha, S., Batatinha, M., Tardy, M., 2010. Genotoxicity and morphological changes induced by the alkaloid monocrotaline, extracted from Crotalaria retusa, in a model of glial cells. Toxicon 55, 105-117.

Sperl, W., Stuppner, H., Gassner, I., Judmaier, W., Dietze, O., Vogel, W., 1995. Reversible hepatic veno-occlusive disease in an infant after consumption of pyrrolizidine-containing herbal tea. European journal of pediatrics 154, 112-116.

Strikwold, M., Spenkelink, B., de Haan, L.H., Woutersen, R.A., Punt, A., Rietjens, I.M., 2017. Integrating in vitro data and physiologically based kinetic (PBK) modelling to assess the in vivo potential developmental toxicity of a series of phenols. Archives of toxicology 91, 21192133.

TGA, 2013. Registered complementary medicines | Therapeutic Goods Administration (TGA).

Tandon, B.N., Tandon, H., Tandon, R., Narndranathan, M., Joshi, Y., 1976. An epidemic of venoocclusive disease of liver in central India. The Lancet 308, 271-272.

Uhl, M., Helma, C., Knasmüller, S., 2000. Evaluation of the single cell gel electrophoresis assay with human hepatoma (HepG2) cells. Mutation Research/Genetic Toxicology and Environmental Mutagenesis 468, 213-225.

van den Berg, S.J., Alhusainy, W., Restani, P., Rietjens, I.M., 2014. Chemical analysis of estragole in fennel based teas and associated safety assessment using the Margin of Exposure (MOE) approach. Food and chemical toxicology 65, 147-154.

Wang, YP., Fu, P.P., Chou, M.W., 2005a. Metabolic activation of the tumorigenic pyrrolizidine alkaloid, retrorsine, leading to DNA adduct formation in vivo. International journal of environmental research and public health 2, 74-79.

Wang, YP., Yan, J., Beger, R.D., Fu, P.P., Chou, M.W., 2005b. Metabolic activation of the tumorigenic pyrrolizidine alkaloid, monocrotaline, leading to DNA adduct formation in vivo . Cancer letters 226, 27-35.

Wang, YP., Yan, J., Fu, P.P., Chou, M.W., 2005c. Human liver microsomal reduction of pyrrolizidine alkaloid $\mathrm{N}$-oxides to form the corresponding carcinogenic parent alkaloid. Toxicology letters $155,411-420$.

Williams, D., Reed, R., Kedzierski, B., Dannan, G., Guengerich, F., Buhler, D., 1989a. Bioactivation and detoxication of the pyrrolizidine alkaloid senecionine by cytochrome P-450 enzymes in rat liver. Drug metabolism and disposition 17, 387-392.

Williams, D.E., Reed, R.L., Kedzierski, B., Ziegler, D.M., Buhler, D.R., 1989b. The role of flavincontaining monooxygenase in the N-oxidation of the pyrrolizidine alkaloid senecionine. Drug metabolism and disposition 17, 380-386.

Xia, Q., Chou, M.W., Edgar, J.A., Doerge, D.R., Fu, P.P., 2006. Formation of DHP-derived DNA adducts from metabolic activation of the prototype heliotridine-type pyrrolizidine alkaloid, lasiocarpine. Cancer letters 231, 138-145.

Xia, Q., Chou, M.W., Kadlubar, F.F., Chan, PC., Fu, P.P., 2003. Human liver microsomal metabolism and DNA adduct formation of the tumorigenic pyrrolizidine alkaloid, riddelliine. Chemical research in toxicology $16,66-73$. 
Xia, Q., Chou, M.W., Lin, G., Fu, P.P., 2004. Metabolic formation of DHP-derived DNA adducts from a representative otonecine type pyrrolizidine alkaloid clivorine and the extract of Ligularia hodgsonnii hook. Chemical research in toxicology 17, 702-708.

Xia, Q., Yan, J., Chou, M.W., Fu, P.P., 2008. Formation of DHP-derived DNA adducts from metabolic activation of the prototype heliotridine-type pyrrolizidine alkaloid, heliotrine. Toxicology letters 178, 77-82.

Xia, Q., Zhao, Y., Von Tungeln, L.S., Doerge, D.R., Lin, G., Cai, L., Fu, P.P., 2013. Pyrrolizidine alkaloid-derived DNA adducts as a common biological biomarker of pyrrolizidine alkaloidinduced tumorigenicity. Chemical research in toxicology 26, 1384-1396.

Xia, Q., He, X., Ma, L., Chen, S. and Fu, P.P., 2018. Pyrrolizidine Alkaloid Secondary Pyrrolic Metabolites Construct Multiple Activation Pathways Leading to DNA Adduct Formation and Potential Liver Tumor Initiation. Chemical research in toxicology, 31(7), pp.619-628.

Xia, Q., Ma, L., He, X., Cai, L. and Fu, P.P., 2015. 7-Glutathione pyrrole adduct: a potential DNA reactive metabolite of pyrrolizidine alkaloids. Chemical research in toxicology, 28(4), pp.615620.

Yan, J., Xia, Q., Chou, M., Fu, P., 2008. Metabolic activation of retronecine and retronecine N-oxideformation of DHP-derived DNA adducts. Toxicology and industrial health 24, 181-188.

Yang, M., Ruan, J., Fu, P.P., Lin, G., 2016. Cytotoxicity of pyrrolizidine alkaloid in human hepatic parenchymal and sinusoidal endothelial cells: Firm evidence for the reactive metabolites mediated pyrrolizidine alkaloid-induced hepatotoxicity. Chemico-biological interactions 243, 119-126.

Yang, Y.-C., Yan, J., Doerge, D.R., Chan, P.-C., Fu, P.P., Chou, M.W., 2001. Metabolic activation of the tumorigenic pyrrolizidine alkaloid, riddelliine, leading to DNA adduct formation in vivo . Chemical research in toxicology 14, 101-109.

Zhang, M., van Ravenzwaay, B., Fabian, E., Rietjens, I.M., Louisse, J., 2018. Towards a generic physiologically based kinetic model to predict in vivo uterotrophic responses in rats by reverse dosimetry of in vitro estrogenicity data. Archives of toxicology 92, 1075-1088. 


\section{Chapter 2}

\section{Risk assessment for pyrrolizidine alkaloids detected in (herbal) teas and plant food supplements}

Lu Chen, Patrick P.J. Mulder, Jochem Louisse, Ad Peijnenburg, Sebas Wesseling, Ivonne M.C.M. Rietjens

Published in: Regulatory Toxicology and Pharmacology (2017) 68: 292-302 


\begin{abstract}
Pyrrolizidine alkaloids (PAs) are plant metabolites present in some botanical preparations, with especially 1,2-unsaturated PAs being of concern because they are genotoxic carcinogens. This study presents an overview of tumour data on PAs and points of departure (PoDs) derived from them, corroborating that the $\mathrm{BMDL}_{10}$ for lasiocarpine represents a conservative PoD for risk assessment. A risk assessment using this $\mathrm{BMDL}_{10}$ and mean levels of PAs reported in literature for (herbal) teas, indicates that consumption of one cup of tea a day would result in MOE values lower than 10000 for several types of (herbal) teas, indicating a priority for risk management for these products A refined risk assessment using interim relative potency (REP) factors showed that based on the mean PA levels, $7(54 \%)$ of 13 types of (herbal) teas and 1 (14\%) of 7 types of plant food supplements (PFS) resulted in MOE values lower than 10000 , indicating a priority for risk management also for these products in particular. This includes both preparations containing PA-producing and non-PA-producing plants. Our study provides insight in the current state-of-the art and limitations in the risk assessment of PAcontaining food products, especially (herbal) teas and PFS, indicating that PAs in food presents a field of interest for current and future risk management.
\end{abstract}




\section{Introduction}

Pyrrolizidine alkaloids (PAs) are naturally occurring chemicals which are produced by a large number of plants (Griffin et al., 2013; NTP, 1986). To date, more than 660 PAs and PA $N$-oxides have been identified from an estimated 6000 plants (Bodi et al., 2014). Especially 1,2-unsaturated PAs are hepatotoxic and considered as genotoxic carcinogens, thus posing a potential risk to human health (Mori et al., 1985). The 1,2-unsaturated PAs can be subdivided by the type of esterification in monoesters, open chained diesters and cyclic diesters (Figure 1). In addition, cyclic diester PAs with an azacyclooctenone, instead of a 1,2-dehydropyrrolizidine ring system, form a special class (Figure 1). Botanical preparations such as (herbal) teas and plant food supplements (PFS) are widely used around the world. However, these preparations have recently been shown to frequently contain toxic PAs (Bodi et al., 2014; IPCS, 1988; Mulder et al., 2015). Bodi et al. (2014) together with the Federal Institute for Risk Assessment (BfR) in Germany who also reported part of the data (BfR, 2013), analysed seven types of herbal drugs (41 samples) and 11 types of (herbal) teas (282 samples) all supposedly to be derived from non-PA-producing plants. The results showed that (herbal) teas can contain significant levels of PAs of up to $5647 \mu \mathrm{g} / \mathrm{kg}$ dry material, while in herbal drugs the total PA level could reach up to $3099 \mu \mathrm{g} / \mathrm{kg}$ (Bodi et al., 2014). The PAs present in the (herbal) teas and PFS were suggested to originate from contamination with PA-containing weeds during harvesting. Mulder et al. (2015) analysed four types of PFS (110 samples) which were derived from non-PA-producing plants, pollen-based supplements (29 samples) and two types of PFS (39 samples) which were derived from PA-producing plants. These authors also analysed eight types of (herbal) teas (169 samples) which were derived from non-PA-producing plants and five types of (herbal) teas (12 samples) derived from PA-producing plants. For (herbal) teas and PFS which were derived from non-PAproducing plants, the level of PAs amounted up to 4805 and $8488 \mu \mathrm{g} / \mathrm{kg}$ in dry material, respectively. For (herbal) teas and PFS which were derived from PA-producing plants, the levels of PAs amounted up to $31101 \mu \mathrm{g} / \mathrm{kg}$ in specific teas (as dry material) and to $2410275 \mu \mathrm{g} / \mathrm{kg}$ in PFS (Mulder et al., 2015) (Note: In March 2017 the PA levels reported for 13 herbal tea samples in the study of Mulder et al. (2015) have been revised. Consequently, EFSA will publish a revised version of the original scientific report on the internet (Patrick P.J. Mulder, patrick.mulder@wur.nl). The revised levels have been taken into account in the calculations). These data showed that the highest values were obtained for (herbal) teas and PFS produced from PA-producing plants, but that PAs are also present in samples produced from non-PA-producing plants. Recently, upon a request form the European Commission, the European Food Safety Authority (EFSA) published a dietary exposure assessment reporting estimates for chronic and acute exposure to PAs using the PA data collected and available in the EFSA Chemical Occurrence database (EFSA, 2016). The data on tea and herbal infusions were submitted by several data providers including five national authorities, and consisted of data provided by tea 
producers and traders organised in Tea \& Herbal Infusions Europe (THIE), as well as data provided by Bodi et al. (2014) and Mulder et al. (2015). EFSA expressed the PA levels per liter of tea infusion as consumed dividing the level in $\mu \mathrm{g} / \mathrm{kg}$ dry material by 75 assuming $100 \%$ extraction of the PAs present in $2 \mathrm{~g}$ of tea into $150 \mathrm{~mL}$ of boiling water. The 95 th percentile values amounted up to $773 \mu \mathrm{g} / \mathrm{kg}$ for individual dry (herbal) tea samples and $55459 \mu \mathrm{g} / \mathrm{kg}$ in PFS, including data from material from PAproducing plants (EFSA, 2016).

In the studies reported by Bodi et al. (2014), Mulder et al. (2015) and EFSA (2016), no risk assessment was presented for the PA levels detected. Given that 1,2-unsaturated PAs are considered to be genotoxic and carcinogenic, the risk assessment can best be done by applying the Margin of Exposure (MOE) approach (EFSA, 2011). To calculate the MOE for a particular compound preferably a $\mathrm{BMDL}_{10}$ from a carcinogenicity study (benchmark dose lower confidence limit for $10 \%$ extra risk on tumour formation above background levels) is normally used as a point of departure (PoD). So far, suitable experimental data to derive such $\mathrm{BMDL}_{10}$ values have only been reported for two PAs, lasiocarpine and riddelliine (NTP, 1978; NTP, 2003). For lasiocarpine EFSA calculated a BMDL 10 of $0.07 \mathrm{mg} / \mathrm{kg}$ bw/day based on data for induction of liver haemangiosarcomas in male rats and used this as PoD for comparison with the estimated dietary exposure resulting from the presence of PAs in retail honey (EFSA, 2011). EFSA indicated that the carcinogenic potency of most PAs present in honey is likely to be lower than that of lasiocarpine and that a risk characterisation using the $\mathrm{BMDL}_{10}$ for lasiocarpine is considered a conservative approach. This is based on the consideration that lasiocarpine is amongst the most toxic of the PAs that have been tested based on the $\mathrm{LD}_{50}$ upon a single intraperitoneal (i.p.) dose (COT, 2008), and the fact that toxicity may be associated with the carcinogenicity. This assumption is in line with the fact that for riddelliine a $\mathrm{BMDL}_{10}$ of $0.18 \mathrm{mg} / \mathrm{kg}$ bw/day was calculated based on the incidence of liver haemangiosarcomas in female rats (EFSA, 2011; NTP, 2003). In addition to lasiocarpine and riddelliine, other PAs, including monocrotaline, clivorine, senkirkine and symphytine, (Figure 1), have been shown to cause tumours in animal bioassays (Hirono et al., 1979; Kuhara et al., 1980; Shumaker et al., 1976). Monocrotaline has been classified as a Group 2B carcinogen (possibly carcinogenic to humans) by the International Agency for Research on Cancer (IARC), while senkirkine and symphytine have been classified as Group 3 (not classifiable as to its carcinogenicity to humans (IARC, 1976; IARC, 1983; IARC, 2002). For these PAs available tumour data are not suitable for dose response modelling and definition of $\mathrm{BMDL}_{10}$ values, but their data could still be used to provide a better estimate of how conservative the use of the $\mathrm{BMDL}_{10}$ of lasiocarpine for risk assessment on 1,2-unsaturated PAs would be. PODs for calculating the MOE in situations where the data do not facilitate dose-response modelling to obtain a $\mathrm{BMDL}_{10}$ are the T25 and/or T10 values, representing the dose levels resulting in 25 or $10 \%$ tumour incidence above back ground levels after lifetime exposure (BfR, 2009; EFSA, 2005). In their opinion on a harmonised approach for risk assessment of substances which are both genotoxic and carcinogenic EFSA already 
indicated that in cases where the data would be unsuitable for deriving a BMDL 10 , use of the T25 is recommended (EFSA, 2005). EFSA also indicated that when using the T25 for calculation of the MOE a value of 25000 instead of 10000 could be used to judge if the MOE indicates a priority for risk management (EFSA, 2005). To facilitate comparison to other genotoxic carcinogens BfR derived an MOE based risk assessment for glycidol and its esters, using the T10 calculated from the T25 by linear extrapolation (multiplying the $\mathrm{T} 25$ by $10 / 25$ ) as an alternative for the $\mathrm{BMDL}_{10}$ for calculating MOE values (BfR, 2009).

In 2013, the BfR presented an initial risk assessment on PAs in (herbal) teas for children and adults using several consumption scenarios (BfR, 2013). In their report, BfR considered the toxic potency of all the PAs as equal when calculating MOE values. It was concluded that it is improbable that short term intake (up to 14 days) poses a health risk for adults and children but that there is a concern for people who frequently drink large quantities of the (herbal) teas. This risk assessment was based on tea samples from the German market and the $\mathrm{BMDL}_{10}$ for lasiocarpine, using several consumption scenarios. BfR indicated that the intake estimates used were likely either overstated or underestimated as they included average group PA content values for all types of teas, while tea consumption reported by consumers and used for the intake estimates is also likely to include other tea varieties that tend to have either lower or higher PA contents. Indeed, it is necessary to consider the fact that some types of (herbal) tea infusion may contain large amounts of PAs, as a relevant food safety issue. This also holds true for PFS.

As already indicated above the toxic potency of different PAs may be different. In view of this, Merz and Schrenk (2016) recently have proposed interim relative potency (REP) factors for PAs, that describe the relative (toxic) potency of each congener compared with the most toxic congener(s) (Merz and Schrenk, 2016). Based on available literature data on in vitro cytotoxicity, genotoxicity in Drosophila, and acute toxicity in rodents (LD50), REP factors were defined for the most relevant structural types of PAs, 1.0 for cyclic di-esters and open-chained di-esters with $7 \mathrm{~S}$ configuration, 0.3 for mono-esters with $7 \mathrm{~S}$ configuration, 0.1 for open-chained diesters with $7 \mathrm{R}$ configuration and 0.01 for mono-esters with 7R configuration (Merz and Schrenk, 2016). It should be noticed that these interim REP values do not take the relative potency of PAs for tumour formation into account. Furthermore, a risk assessment based on the PA levels in (herbal) teas or PFS, taking the interim REP factors into account, has so far not been performed. Given this situation the aims of the present study were i) to analyse the literature available on PA induced tumour incidences, to define PODs for PAs other than lasiocarpine and to further evaluate the use of the $\mathrm{BMDL}_{10}$ of lasiocarpine for risk assessment of 1,2-unsaturated PAs and ii) to present a risk assessment on the use of (herbal) teas and PFS containing PAs using the new data presented by Bodi et al. (2014), Mulder et al. (2015) and EFSA (2016) taking into account the interim REP factors. 


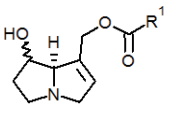

A

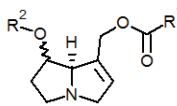

B

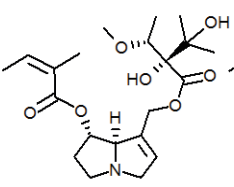

Lasiocarpine

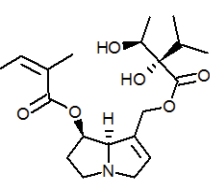

Symphytine
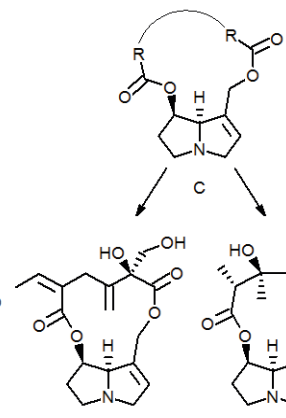

Riddelliine

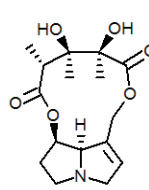

Monocrotaline
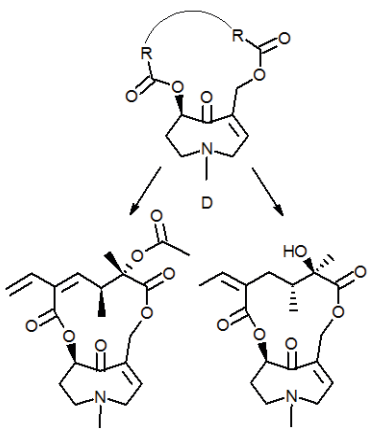

Senkirkine

Figure 1. Chemical structures of PAs subdivided by the type of esterification: 1,2 dehydropyrrolizidine with A: monoester; B: open chained diester; C: cyclic diester. D: azacyclooctenone cyclic diester).

\section{Materials and Methods}

\section{Literature search}

To obtain the required data on PA-induced tumour incidences, a literature search was performed using Google Scholar by combining the search terms: 'pyrrolizidine alkaloids', 'carcinogenic' and 'rats' or 'mice'. References that met all the search criteria were collected. From these studies, the essential information, such as number of animals, species, gender, dose, tumour data and experimental time, was extracted for further evaluation. Based on the carcinogenicity data, potential PoDs were defined as described hereafter.

\section{PoDs definition for risk assessment}

Using the BMD method, the lower confidence limit of the benchmark dose (BMD) giving a 10\% extra cancer incidence $\left(\mathrm{BMDL}_{10}\right)$ was determined as a preferred PoD for the MOE approach. BMD modelling was performed using all models for dichotomous data of the Environmental Protection Agency (EPA)'s Benchmark Dose Software (BMDS) version 2.6 applying default settings. All models that met the requirements for acceptance $\left(\mathrm{P}\right.$-value $>0.05$ and $\left.\mathrm{BMD}_{10} / \mathrm{BMDL}_{10}<10\right)$ were considered for the determination of $\mathrm{BMDL}_{10}$ values choosing the lowest $\mathrm{BMDL}_{10}$ value for further assessment. To calculate $\mathrm{BMDL}_{10}$ values, data should preferably refer to three experimental doses administrated to rats or mice, with at least 50 animals per group and a two-year experimental period (Edler et al., 2014). However, in some experiments, the study was terminated before completion of a two-year (104 weeks) period and the actual treatment period was even shorter. For these studies an adjustment of the dose level was performed using the formula: dose $\times($ weeks of treatment/104) $\times($ weeks of observation/104) (ECHA, 2008), resulting in the dose levels used for deriving the POD. For example for the data on clivorine, the experimental dose ( $5 \mathrm{mg} / \mathrm{kg}$ bw/day given for 340 days (= 49 weeks), with experiment termination at 480 days (= 69 weeks) was adjusted by multiplying the dose by $(49 / 104) \times(69 / 104)$, giving a dose of $1.56 \mathrm{mg} / \mathrm{kg}$ bw/day. 
In case the data are insufficient for the calculation of a $\mathrm{BMDL}_{10}$, EFSA has recommended the $\mathrm{T} 25$ as an alternative point for MOE calculation. T25 values are calculated by dividing the extra risk (\% incidence in the dose group minus \% incidence in the control group) by the non-affected fraction in the control population (100 minus background response in \%), and converting the dose level causing this tumour incidence to the dose level causing $25 \%$ extra tumour incidence by linear extrapolation. For example, if the incidence in the control group was $5 / 50(10 \%)$ and in the $30 \mathrm{mg} / \mathrm{kg}$ bw/day group $(30 / 50(60 \%))$, the extra risk is $[(60-10) /(100-10)] / 100=55.6 \%$ and the $\mathrm{T} 25=25 / 55.6 \times 30 \mathrm{mg} / \mathrm{kg}$ $\mathrm{bw} /$ day $=13.5 \mathrm{mg} / \mathrm{kg}$ bw/day (Benford et al., 2010). T10 values were calculated from the T25 by linear extrapolation, multiplying by 10/25. It is of interest to note that one could argue that statistically a T10 would compare to a $\mathrm{BMD}_{10}$ rather than to a $\mathrm{BMDL}_{10}$ but at the current state of the art the T25 with a MOE cut off value of 25000 and the T10 with a MOE cut off value of 10000 (also applied when using the $\mathrm{BMDL}_{10}$ ) are used as PODs when a $\mathrm{BMDL}_{10}$ is not available.

\section{Estimated Daily Intake (EDI) Calculation}

A number of different data sets were used for estimating exposure including those reported by Bodi et al. (2014), Mulder et al. (2015) and EFSA (2016). The mean and maximum values of total PA content for different types of (herbal) teas or PFS obtained from Bodi et al. (2014) are summarised in Supplementary Table S5 and S9. Instead of maximum PA levels, the EFSA report gives the 95th percentile values (EFSA, 2016). These data together with the mean values of total PA contents for (herbal) teas and PFS are summarised in Supplementary Table S6 and S10. Only the data reported by Mulder et al. (2015) were presented at the level of detail needed for 'adjustment' of the PA levels by REP factors. The mean and maximum total PA content for different (herbal) teas or PFS obtained from Mulder et al. (2015) are summarised in Supplementary Table S7 and S11 and the corresponding data adjusted by REP factors and are presented in Supplementary Table S8 and S12. The total PA levels that were used in this study were the ones based on the lower bound approach, meaning that only quantified levels were taken into account and that non-detected PAs were ignored. To perform the risk assessments, the daily PA intake resulting from use of the (herbal) teas was estimated assuming daily consumption of the amount of PAs present in $2 \mathrm{~g}$ tea, corresponding to one cup of tea, as described before (BfR, 2013). Due to the wide range of the recommended daily intake between PFS products (COT, 2003), it is difficult to achieve a consensus estimate on the general PFS daily intake. In this study, the daily PA intake resulting from use of PFS was estimated by assuming a dose of 200 mg PFS. This dose reflects the low end of intake of botanical supplements (Van Den Berg et al., 2011). The estimated daily intakes (EDIs) were calculated using a default body weight of $70 \mathrm{~kg}$ for an adult as proposed by EFSA (EFSA, 2012).

\section{Calculation of the Margin of Exposure}

The MOE was calculated by dividing the selected PoD (BMDL 10 or T10 as indicated) by the EDI. To evaluate the possible health risk and priority for risk management actions an MOE value of 10000 
was applied. This value of 10000 includes a factor of 100 , consisting of a factor of 10 for possible inter-species differences, and a factor of 10 for differences between human individuals. Furthermore it includes an additional factor of 10 to account for inter-individual human variability in cell cycle control and DNA repair and a factor 10 because the MOE is based on the BMDL 10 which is not a no effect level (EFSA, 2005). An MOE value of 10000 or higher, based on animal cancer bioassay data, is considered to be a low concern from a public health standpoint, while a value lower than 10000 might raise a potential concern for human health (EFSA, 2005). When using the T25 for calculation of the MOE a value of 25000 instead of 10000 should be used to judge if the MOE indicates a safety concern (EFSA, 2005). As proposed by the BfR a value of 10000 could be considered when using a $\mathrm{T} 10$ to facilitate comparison to the $\mathrm{BMDL}_{10}$ (BfR, 2009).

\section{Results}

\section{Carcinogenicity data and PoDs for PAs}

Table 1 provides an overview of the carcinogenicity data of PAs as present in the literature, describing the incidence of liver haemangiosarcomas for lasiocarpine and riddelliine, pulmonary adenocarcinomas for monocrotaline, liver neoplastic nodules for clivorine, liver cell adenomas for senkirkine and hemangioendothelial sarcoma of the liver for symphytine, in all cases observed in rats. Only the data for lasiocarpine, and riddelliine appeared suitable for BMD modelling, as was also concluded by EFSA (EFSA, 2011). The results of a BMD analysis of these data confirmed the $\mathrm{BMDL}_{10}$ values previously reported by EFSA, of 0.07 and $0.18 \mathrm{mg} / \mathrm{kg}$ bw/day for lasiocarpine and riddelliine, respectively, based on the data for induction of liver haemangiosarcoma in male and female rats (Table 2). Based on the experimental doses, the duration of the studies and tumour incidences, T25 and T10 values for induction of the different tumour types were calculated for monocrotaline, clivorine, senkirkine and symphytine (Supplementary Tables S1-S4). Table 2 presents an overview of these values listing the lowest $\mathrm{BMDL}_{10}$ and $\mathrm{T} 10$ values for the tumour types that can be used as a preferred PoD for the further assessment. For monocrotaline, the T10 value was 1.5 $\mathrm{mg} / \mathrm{kg}$ bw/day based on a study using subcutaneous (s.c.) administration of monocrotaline inducing pulmonary adenocarcinoma in male rats (Shumaker et al., 1976). The T10 value for clivorine was 0.31 $\mathrm{mg} / \mathrm{kg}$ bw/day based on a rat study in which clivorine was administered in drinking water, inducing an increased incidence of liver neoplastic nodules in the liver in male and female rats (Kuhara et al., 1980). The T10 values were $2.4 \mathrm{mg} / \mathrm{kg}$ bw/day for senkirkine and $4.0 \mathrm{mg} / \mathrm{kg}$ bw/day for symphytine, based on the incidence of liver cell adenomas and liver haemangioendothelial sarcoma, respectively, in male rats exposed via i.p. injection (Hirono et al., 1979). T10 values derived for lasiocarpine and riddelliine amounted to 0.16 and $0.09 \mathrm{mg} / \mathrm{kg}$ bw/day and were thus somewhat different from the $\mathrm{BMDL}_{10}$ values derived from the same data. Based on comparison of the BMDL 10 and $\mathrm{T} 10$ values shown in Table 2, it can be concluded that the BMDL 10 of $0.07 \mathrm{mg} / \mathrm{kg}$ bw/day for lasiocarpine, appears to be the lowest PoD of all values obtained. This value is 3 to 60 -fold lower than the PoDs 
obtained for related 1, 2-unsaturated PAs (Table 2). This indicates that lasiocarpine is the most toxic PA compared to those for which tumour data are available and that therefore a risk characterisation based on the $\mathrm{BMDL}_{10}$ for lasiocarpine can indeed be considered a conservative approach (EFSA, 2011). However, given the fact that for several PAs the PoD seems to be more than ten-fold higher than the $\mathrm{BMDL}_{10}$ for lasiocarpine, it should be kept in mind that, depending on the nature of the PAs actually present, use of the $\mathrm{BMDL}_{10}$ for lasiocarpine may result in MOE values that may be too low. 


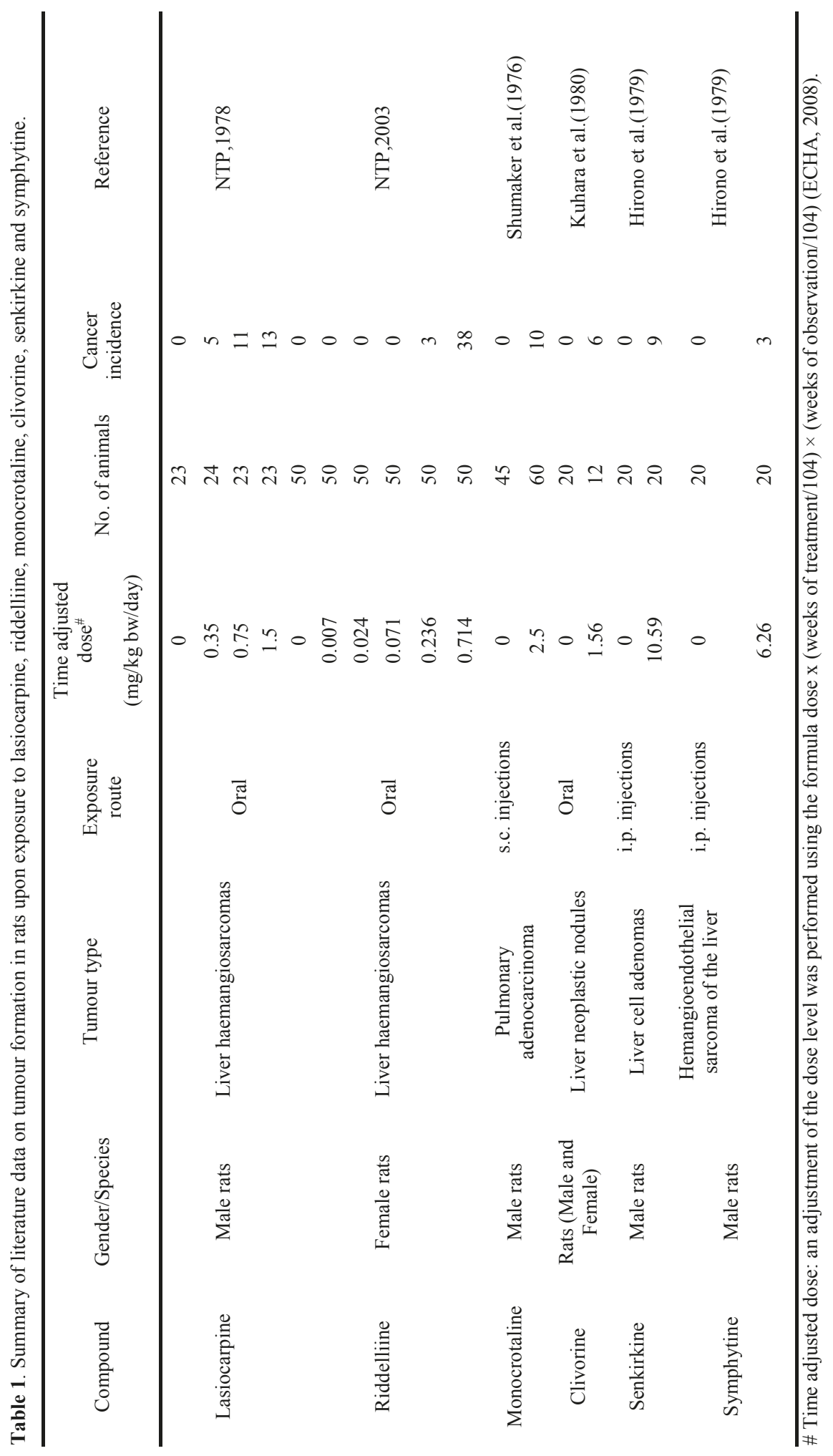




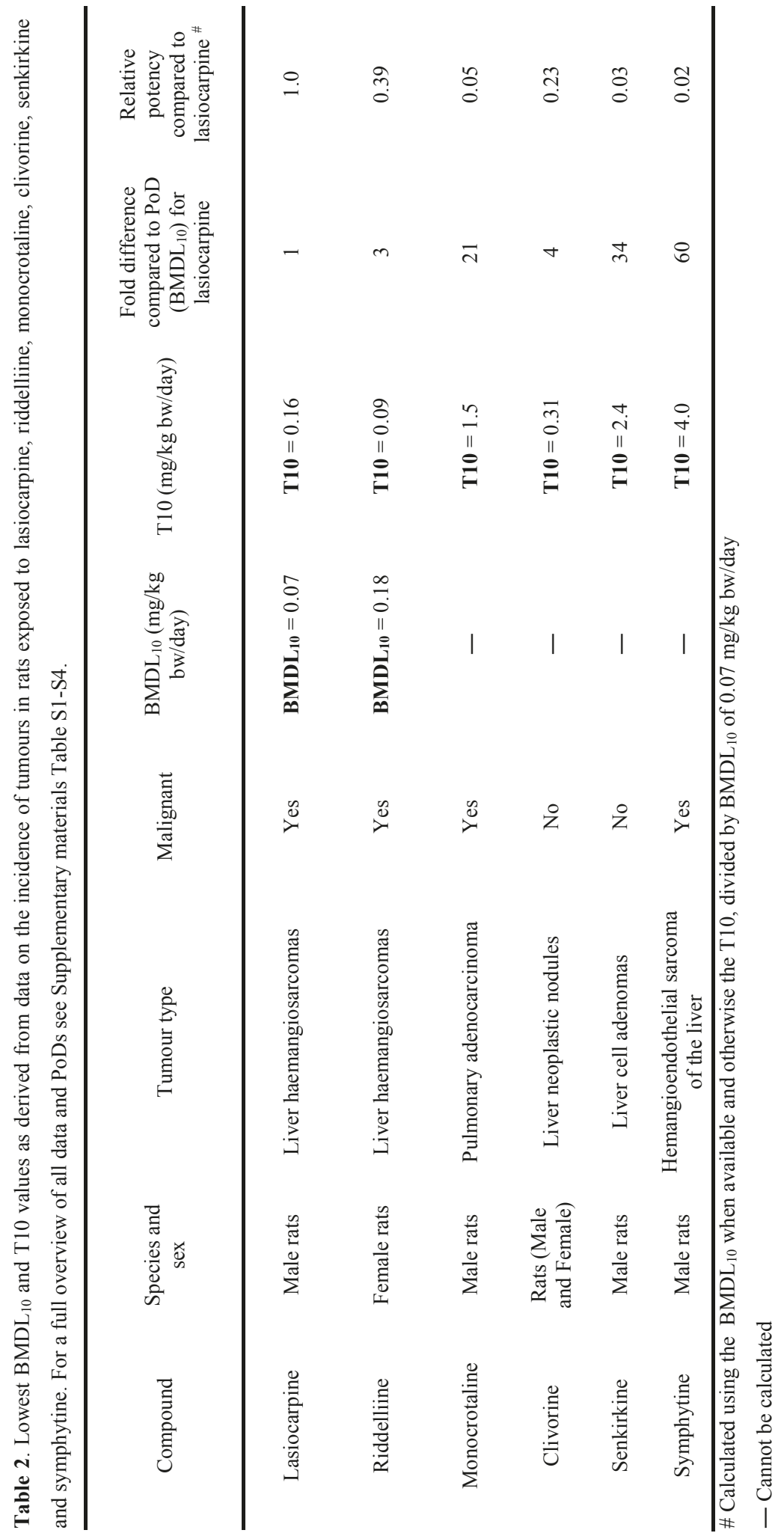




\section{Estimated Daily Intakes (EDIs) and risk assessment of PAs from consumption of (herbal)}

teas

Based on the mean and maximum PA levels reported by Bodi et al. (2014) (Supplementary Table S5), the EDI and MOE values for a total of 11 types of (herbal) teas (with at least 9 samples per type of tea) were calculated assuming daily consumption of one cup of tea (using $2 \mathrm{~g}$ dry tea). Figure 2 presents the resulting MOE values for the different types of (herbal) teas based on the $\mathrm{BMDL}_{10}$ for lasiocarpine. The MOE values ranged from 430 to around 47500 upon consumption of one cup of (herbal) tea per day. Overall, the MOE values vary significantly among the different types of (herbal) teas. Depending on the use of mean or maximum PA levels, the types of tea with MOE values below 10000 , amount to four $(36 \%)$ and nine (82\%) of the 11 types of (herbal) teas, respectively. More specifically, based on the mean PA level, MOE values lower than 10000 were found for chamomile, black, rooibos and melissa teas (Figure 2A). In addition to those teas, the MOE values of mixed herbal, peppermint, green, fennel and nettle teas were below 10000 when taking the maximum PA levels into account (Figure 2B). It is of interest to note that all (herbal) teas were derived from non-PA-producing plants.
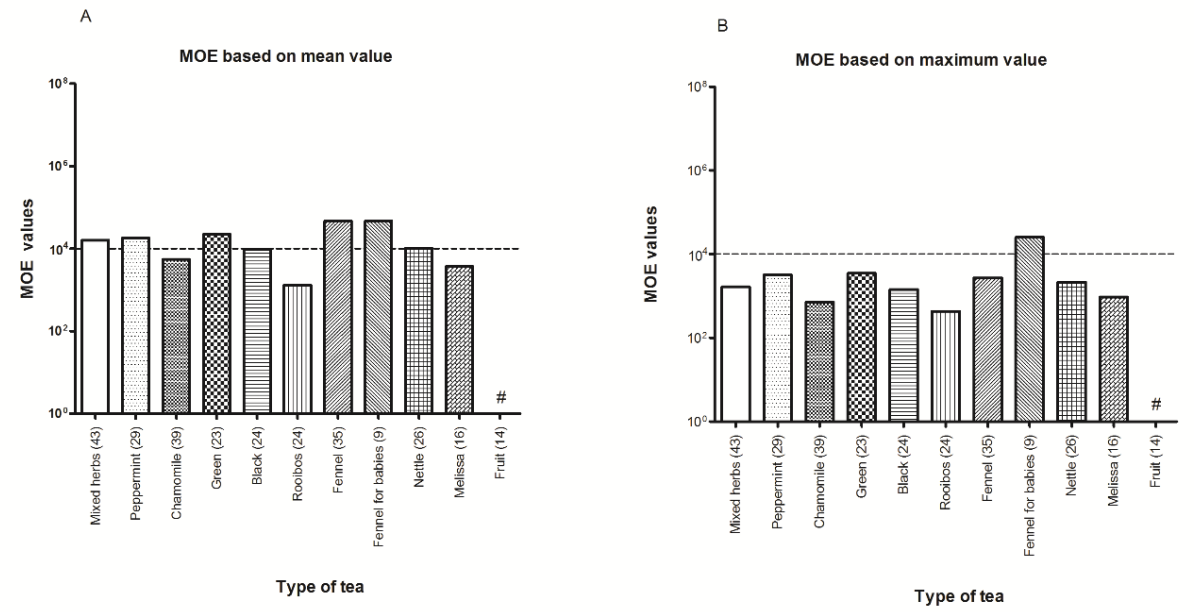

Figure 2. The MOE values for 11 different types of herbal teas obtained for daily consumption of one cup of tea per day using A) mean, B) maximum PA levels reported by Bodi et al. (2014). The number of teas analysed in each category is given in brackets. \# PA content $<$ LOQ. The BMDL 10 for lasiocarpine is used as PoD.

Figure 3 presents MOE values calculated by using the mean and 95th percentile of total PA values based on the type of (herbal) teas that were reported by EFSA (2016), including the data reported by Bodi et al. (2014) and Mulder et al. (2015). The EDI and MOE values were calculated by assuming consumption of one cup of tea per day ( $2 \mathrm{~g}$ dry weight $)$ and are presented in the Supplementary Table S6. Based on the mean PA levels, most types of teas have MOE values higher than 10000 except peppermint and rooibos tea (Figure 3A). When considering the 95th percentile PA levels, in addition to peppermint and rooibos tea, another four types of tea, including tea and herbs, black, green and 
chamomile teas, were found to result in MOE values below 10000 (Figure 3B). Again, all of these are from non-PA- producing plants.
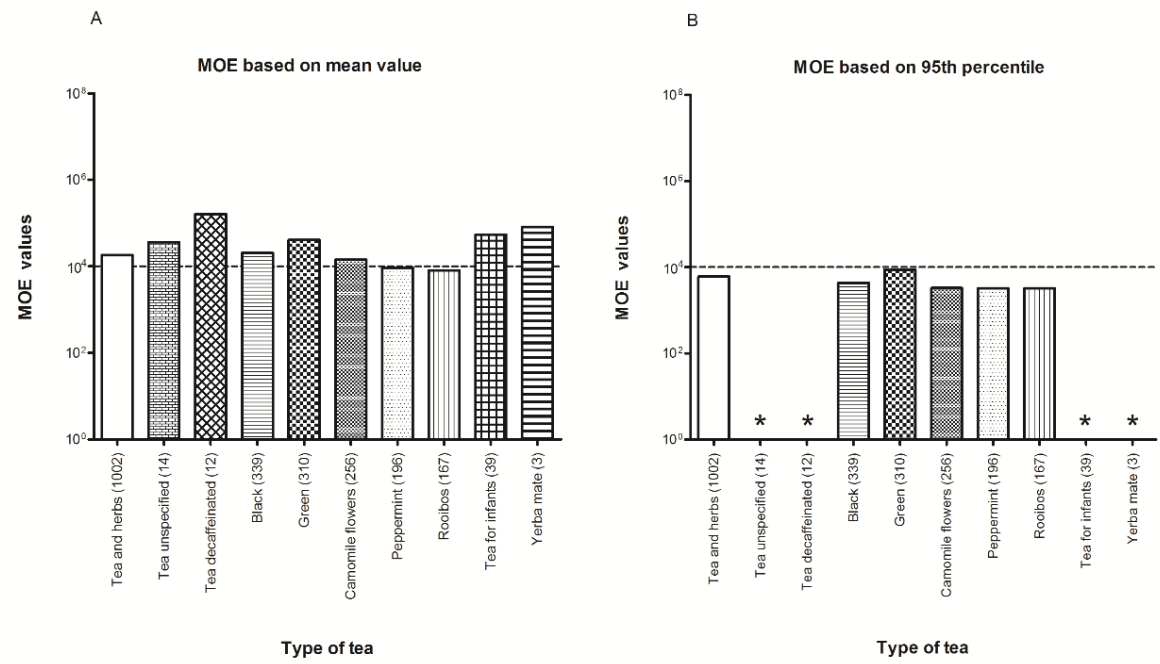

Figure 3. The MOE values for 10 different types of herbal teas obtained for daily consumption of one cup of tea per day using A) mean, B) 95th percentile PA levels reported by EFSA (2016). The number of teas analysed in each category is given in brackets. * Data are not available in EFSA (2016). The BMDL 10 for lasiocarpine is used as PoD.

\section{Estimated Daily Intakes (EDIs) and risk assessment of PAs from consumption of (herbal) teas, taking relative potencies into account}

The data reported by Mulder et al. (2015) (see note above on the recent revision of the report) also include information on the individual PAs detected, which is essential when taking the different potencies of the PAs into account by applying REP factors. The individual PA levels reported by Mulder et al. (2015) were recalculated using the interim REP factors, reported by Merz and Schrenk (Merz and Schrenk, 2016), to adjust the mean and maximum total PA levels and to calculate the EDI values for 13 types of (herbal) teas in relative potency assuming consumption of one cup of tea. These data without and with taking the REP factors into account are presented in the Supplementary Table S7 and S8, respectively. The MOE values thus obtained, either with or without taking the REP factors into account, are presented in Figure 4. After REP factor correction, the MOE values ranged from 460 to around 445000 upon consumption of one cup of (herbal) tea per day. Based on the mean and maximum PA levels and with taking the REP factors into account, the types of teas with MOE values below 10000 , amounted to $7(54 \%)$ and $8(62 \%)$ out of a total of 13 types of (herbal) teas, respectively. More specifically, based on the mean PA levels and REP correction, MOE values less than 10000 were found for peppermint, rooibos, black, green, mixed herbal, borago and coltsfoot tea (Figure 4A). When considering maximum PA levels, also camomile flower resulted in MOE values 
below 10000 (Figure 4B). Of these teas, chamomile flowers, peppermint, rooibos, black, green and mixed herbs are derived from non-PA-producing plants, whereas borago and coltsfoot are derived from PA-producing plants. It is of interest to note that the MOE values for chamomile flowers, peppermint, rooibos, black, green and mixed herbs increase by only about 1 to 2 fold after applying REP factors. This limited effect is due to the fact that the major PAs in these teas were senecionine, retrorsine and seneciphylline (Mulder et al., 2015) for which the interim REP factors are 1.0. In contrast, for teas derived from PA containing plants such as borago, gromwell, eupatorium and lungwort, the MOE values increased by 30 to 100 fold after applying REP factors (Figure 4), due to the fact that the major PAs in these preparations were intermedine and lycopsamine, for which the proposed REP factors are 0.01 (Merz and Schrenk, 2016).
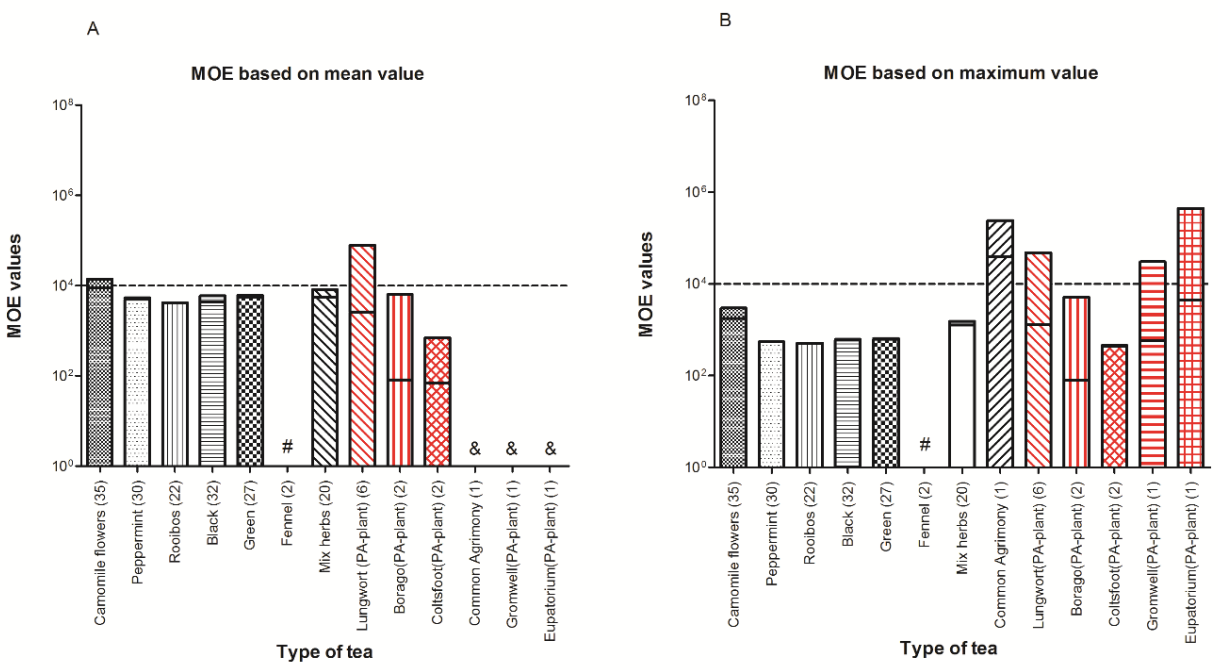

Figure 4 . The MOE values of 13 different types of herbal teas obtained for daily consumption of one cup of tea per day using A) mean, B) maximum PA levels, reported by Mulder et al. (2015). The number of teas analysed in each category is given in brackets. The area above the black solid line inside each bar represents the increment of MOE values after the REP factor correction of the individual PA levels. The bars filled inside with black lines are corresponding to the samples that were derived from non-PA-producing plants and the ones with red lines are corresponding to the samples that were derived from PA-producing plants. \# PA content $<$ LOQ. \& No mean values calculated, as only one sample was measured. These results were treated as maximum values. The $\mathrm{BMDL}_{10}$ for lasiocarpine is used as PoD.

\section{Estimated Daily Intakes (EDIs) and risk assessment of PAs from consumption of plant food supplements}

The EDI and MOE values for seven types of PFS for which PA levels were reported by Bodi et al. (2014) are presented in the Supplementary Table S9. The different types of PFS with MOE values lower or higher than 10000 upon daily consumption of $200 \mathrm{mg}$ PFS per day are presented in Figure 5. 
Overall, the MOE values vary considerably among the types of PFS, ranging from 7900 to around 17 500 000. Based on the mean or maximum PA levels reported, none and one (14\%) of a total of seven types of PFS resulted in an MOE value below 10 000, respectively (Figure 5). Based on the maximum PA levels, the MOE values of PFS containing anise that were derived from non-PA-producing plants were lower than 10000 (Figure 5B).

Figure 6 presents MOE values calculated by using mean and the 95th percentile of total PA levels of PFS that were reported by EFSA (2016). The EDI and MOE values were calculated by assuming consumption of $200 \mathrm{mg}$ PFS per day and presented in the Supplementary Table S10. According to either the mean or the 95th percentile PA values, the plant extract formula supplements resulted in an MOE value below 10000 (Figure 6).

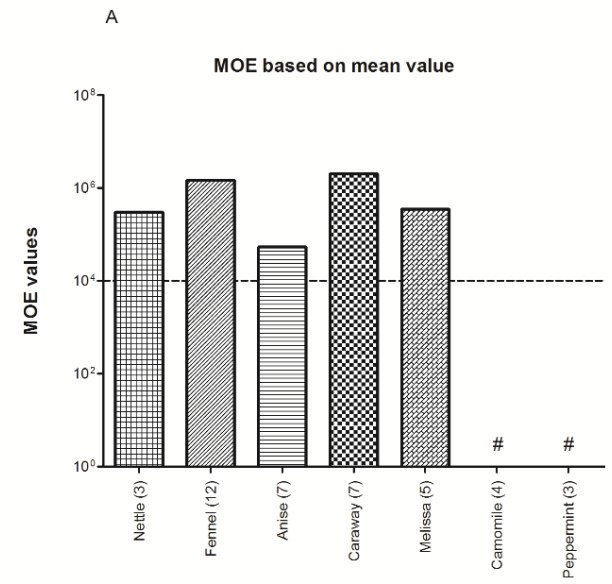

Type of PFS
B

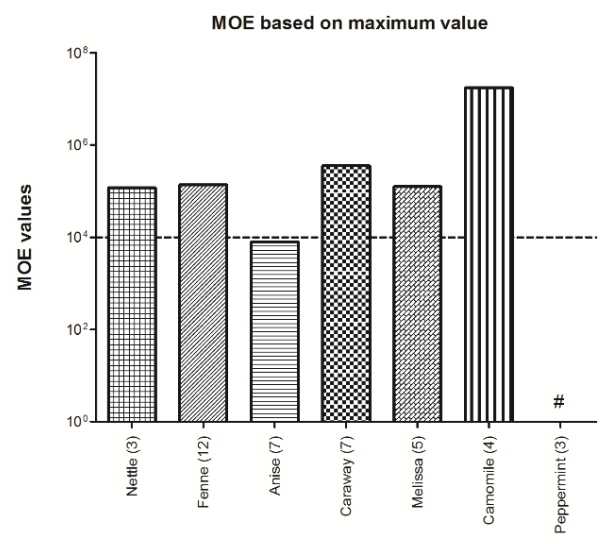

Type of PFS

Figure 5. The MOE values of 7 different types of PFS obtained for daily consumption of $200 \mathrm{mg}$ of PFS per day using A) mean, B) maximum PA levels reported by Bodi et al. (2014). The number of PFS analysed in each category is given in brackets. \# PA content $<\mathrm{LOQ}$. The $\mathrm{BMDL}_{10}$ for lasiocarpine is used as PoD. 


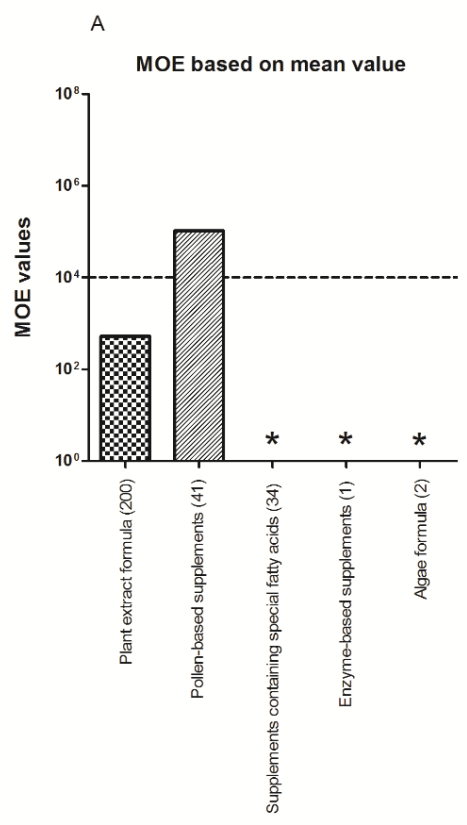

Type of PFS

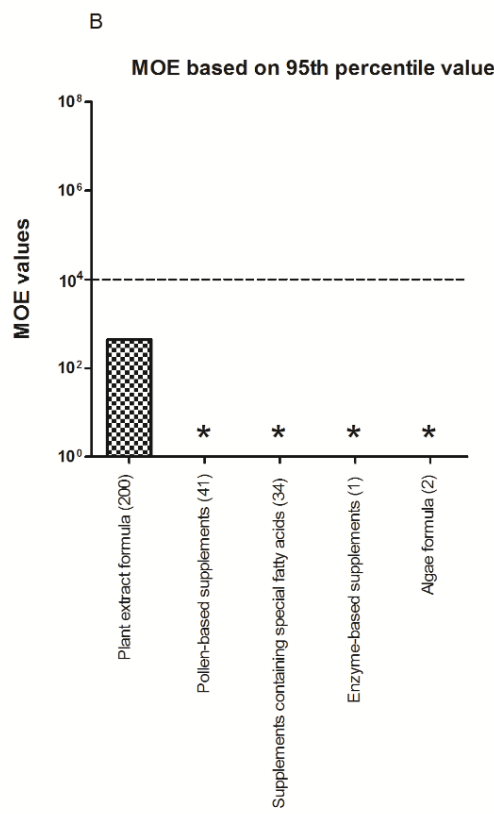

Type of PFS

Figure 6. The MOE values for 5 different types of PFS obtained for daily consumption of 200 mg dry weight per day using A) mean, B) 95th percentile PA levels reported by EFSA (2016). The number of PFS analysed in each category is given in brackets. * Data is are not available in EFSA (2016). The BMDL 10 for lasiocarpine is used as PoD.

\section{Estimated Daily Intakes (EDIs) and safety assessment of PAs from consumption of plant food supplements taking relative potencies into account}

The EDI and MOE values, without and with REP factor correction for seven types of PFS reported by Mulder et al. (2015) are presented in the Supplementary Table S11 and S12. The MOE values upon daily consumption of $200 \mathrm{mg}$ PFS per day are presented in Figure 7. Overall, and taking the relative potencies into account, the MOE values vary substantially among the types of PFS, ranging from 1000 to around 983 000. Based on the mean and maximum PA levels, when taking the relative potencies into account, only the supplements prepared from PA-plant extract formula resulted in an MOE value below 10 000, accounting for one (14\%) of the seven types of PFS investigated (Figure 7). It is of interest to note that only a small proportion of the PFS analysed contained lasciocarpine: two PA-plant extract formulas out of $18(11 \%)$, three out of 75 samples (4\%) of non-PA-producing plants extract formulas and none of the 29 pollen-based PFS. Supplements containing special fatty acids, derived from PA-producing plants did not contain measurable amounts of PAs due to the fact that in the production process the PAs are effectively removed by refinement of the oil. These products therefore comply with legislation in the Netherlands that states that preparations made from PA-containing 
plants shall not contain more than $1 \mu \mathrm{g} / \mathrm{kg}$ PAs (Warenwetbesluit kruidenpreparaten 2001) (Supplementary materials Table S11 and S12). Notably, the MOE values of pollen-based supplements, plant extract formula made of non-PA-producing plant material, valerian, St John's wort or PAproducing plant extract material increased after applying REP factors by about 6-, 4-, 1-, 10- and 65fold, respectively. This can be ascribed to the fact that the major PAs present in these PFS were lycopsamine, intermedine and echimidine (Mulder et al., 2015), which were reported to have REP factors of 0.01, 0.01 and 0.1, respectively (Merz and Schrenk, 2016).
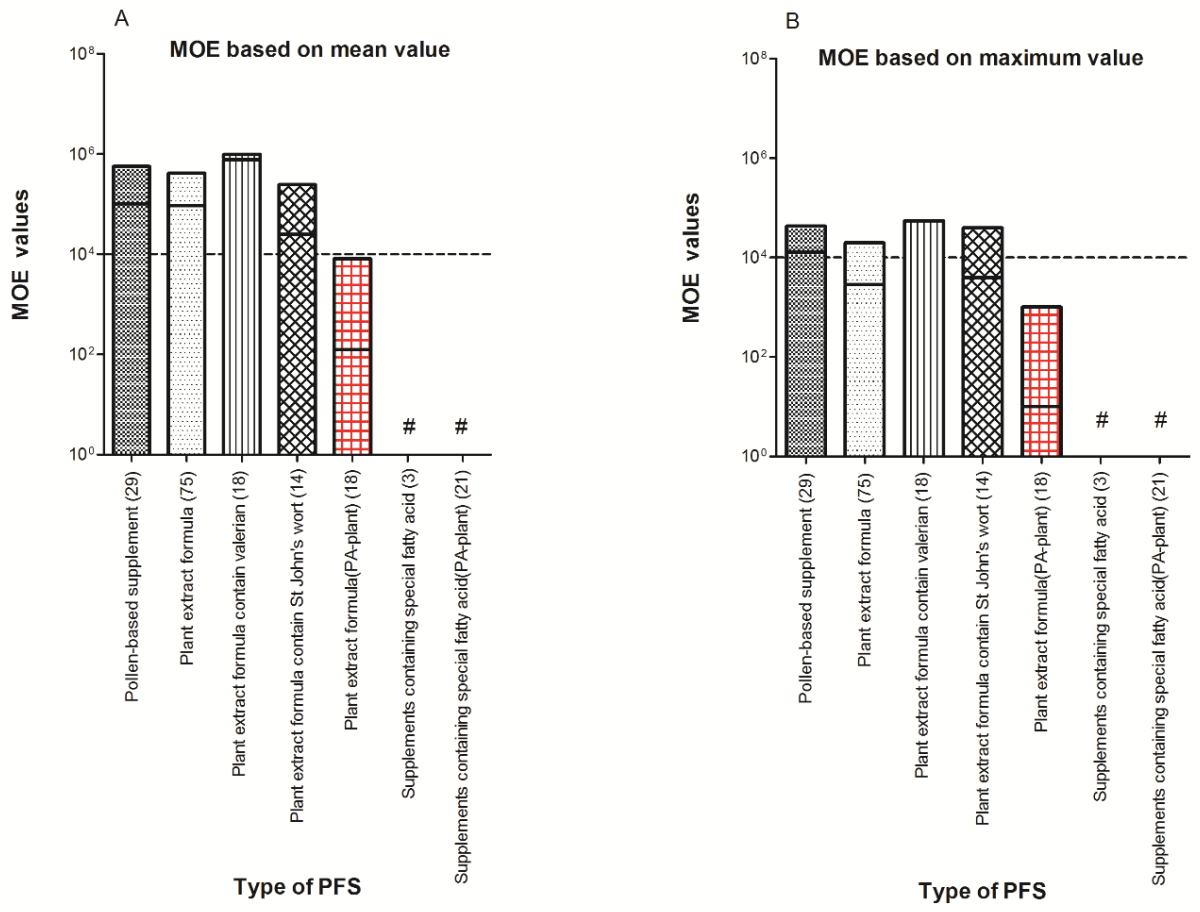

Figure 7. The MOE values for 7 different types of PFS obtained for daily consumption of 200 mg of dry weight per day using A) mean, B) maximum PA levels, reported by Mulder et al. (2015). The number of PFS analysed in each category is given in brackets. The area above the black solid line inside each bar represents the increment of MOE values after the REP factor correction of the individual PA levels. The bars filled inside with black lines are corresponding to the samples that are derived from non-PA-producing plants and the ones with red lines are corresponding to the samples that are derived from PA-producing plants. \#PA content $<\mathrm{LOQ}$. The $\mathrm{BMDL}_{10}$ for lasiocarpine is used as $\mathrm{PoD}$.

\section{Discussion}

In the present study, first a literature review on tumour data on PAs was performed to obtain a better insight in PoDs available for risk assessment of PAs and the extent to which the BMDL 10 of lasiocarpine currently used presents a worst case PoD. Data on lasiocarpine and riddelliine were 
available and suitable for dose-response modelling, providing the $\mathrm{BMDL}_{10}$ values reported previously by EFSA of 0.07 and $0.18 \mathrm{mg} / \mathrm{kg}$ bw/day, respectively (EFSA, 2011). In addition, tumour data for monocrotaline, clivorine, senkirkine and symphytine were retrieved that were unsuitable for a BMD modelling but allowed derivation of a T25 and T10. In a carcinogenicity study of monocrotaline in male rats, there were treatment-related increases in a number of tumours, principally liver cell carcinomas and pulmonary adenocarcinomas (Shumaker et al., 1976). The T10 value for liver cell carcinomas of $3.0 \mathrm{mg} / \mathrm{kg}$ bw/day was higher than the T10 for pulmonary adenocarcinomas $(1.5 \mathrm{mg} / \mathrm{kg}$ bw/day). The latter was thus selected as the preferred PoD for monocrotaline. Clivorine induced an increased incidence of haemangioendothelial sarcomas and neoplastic nodules in the liver of rats (Kuhara et al., 1980). Although the T10 value of liver hemangioendothelial sarcoma was higher than that derived for liver neoplastic nodules ( $0.96 \mathrm{vs} 0.31 \mathrm{mg} / \mathrm{kg}$ bw/day), liver neoplastic nodules, being non-malignant tumours, may be less suitable for the risk assessment thus leaving the T10 of 0.96 $\mathrm{mg} / \mathrm{kg}$ bw/day as the PoD for clivorine. Senkirkine increased the incidence of liver cell adenomas with a T10 of $2.4 \mathrm{mg} / \mathrm{kg}$ bw/day and symphytine induced liver haemangioendothelial sarcoma, a type of malignant tumour, with a lower T10 value than that for liver cell adenomas $(4.0 \mathrm{mg} / \mathrm{kg}$ bw/day vs 12 $\mathrm{mg} / \mathrm{kg}$ bw/day). All these tumour data appeared inadequate for dose response modelling, because only one level of dosing was included in the studies. The T25 has been suggested by EFSA as an alternative PoD in case a BMDL 10 cannot be calculated (EFSA, 2005) and was recently used for glycidol and its esters (EFSA, 2016). For these same compounds BfR used the T10 as an alternative to make an MOE based risk assessment, because it better allows a comparison to the BMDL 10 than the T25 (BfR, 2009). Our results showed that the T10 values of monocrotaline, clivorine, senkirkine and symphytine were 21-, 4-, 34- and 60- fold higher, respectively, than the $\mathrm{BMDL}_{10}$ value of $0.07 \mathrm{mg} / \mathrm{kg} \mathrm{bw} / \mathrm{day}$ for lasiocarpine (Table 2). For comparison, T10 values for lasiocarpine and riddelliine were also calculated by using the data presented in Table 1 . The T10 values amounted to 0.16 and $0.09 \mathrm{mg} / \mathrm{kg}$ bw/day, respectively, and were thus somewhat different from the $\mathrm{BMDL}_{10}$ values derived from the same data (Table 2). However these values are still in line with the conclusion that the $\mathrm{BMDL}_{10}$ for lasiocarpine of $0.07 \mathrm{mg} / \mathrm{kg}$ bw/day is the lowest PoD and thus seems a conservative estimate when applied for other PAs. Given the consideration that ultimately most 1,2-unsaturated PAs lead to the same type of tumour, namely angiosarcomas, and produce the same type of reactive metabolite, a 6,7dihydro-7-hydroxy-1-hydroxymethyl-5H-pyrrolizine (DHP) analogue, the Committee on Carcinogenicity of Chemicals in Food, Consumer Products and the Environment (COT) agreed that the $\mathrm{BMDL}_{10}$ of lasiocarpine, the most potent $\mathrm{PA}$, would be an appropriate basis for the MOE approach for risk assessment of PAs (COT, 2008). Also EFSA considered lasiocarpine to be the most toxic of the PAs that have been investigated (EFSA, 2011) and BfR as well used the BMDL 10 of lasiocarpine in their risk assessment of PAs in teas (BfR, 2013). The literature overview presented here strongly indicates that this approach using the $\mathrm{BMDL}_{10}$ of $0.07 \mathrm{mg} / \mathrm{kg}$ bw/day for risk assessment of combined exposure to 1,2-unsturated PAs is a conservative approach since tumour data available for other PAs 
so far point at PoDs that are higher. It is important to notethat some of these PoDs were derived from studies where the route of administration was not oral but subcutaneous (s.c.) or intraperitoneal (i.p.) (Table 1). Given that bioavailability by these other routes may be different and perhaps higher than by the oral route, this could provide another reason why the use of the $\mathrm{BMDL}_{10}$ for lasiocarpine for these other PAs might be a conservative approach. It should be noted, that the cancer studies used were carried out in different laboratories, using different species strains, genders, duration and other circumstances. This should be kept in mind when comparing and using the different PoDs' (MacGregor et al., 2015).

Based on PA levels reported by Bodi et al. (2014), when calculating MOE values based on mean and maximum level of PAs, most types of (herbal) teas resulted in MOE values below 10000 . These results indicate a potential risk for human health, even for (herbal) teas derived from non-PAcontaining botanicals. The presence of PAs detected in teas made from non-PA-producing plants has been suggested to be due to (unintentional) contamination with PA-containing weeds during harvesting, processing or blending of the teas (Bodi et al., 2014; Shimshoni et al., 2015). Better production and manufacture practices will likely result in a reduction of the PA contamination in the end products. For PFS, based on the mean PA level, only one (14\%) of in total seven types of PFS would result in EDI values that give rise to an MOE value below 10000 .

To provide a more refined estimate for PA risk assessment, mean and maximum PA levels as reported by Mulder et al. (2015) were used to investigate the effect of applying recently proposed interim REP factors to obtain refined EDI and MOE estimates. The MOE values obtained without REP factor correction were generally lower than those with REP factor correction, especially for the PFS because they contained as major PAs congeners with REP values of 0.01 and 0.1 , while in the teas the major PAs were those with REP values of 1.0 , resulting only in a limited effect.

It is important to stress that the risk assessment presented here is a conservative estimate in various aspects. First of all, the method of preparing the hot water extracts of (herbal) teas and PFS to determine the PA levels in the procedure applied by Mulder et al. (2015) is stated to be in accordance with the real situation to make a cup of tea, but it should be noted that the method included grinding the (herbal) teas and (part of the) herbal food supplements with dry ice before extraction. Grinding the tea and PFS material with dry ice and to a small particle size $(\leqslant 500 \mu \mathrm{m})$ can be expected to facilitate extraction as compared to using the intact samples as such, with the latter being more representative of daily practice. Extraction without the grinding step could result in lower extraction efficiency and thus, lower EDI and higher MOE values, reducing the concern. Previous studies on the risks of exposure to alkenylbenzenes from consumption of fennel based teas by van den Berg et al. (2014) and Raffo et al. (2011) reported that hot water extraction of compounds of concern from comminuted fennel fruits is indeed more efficient than from the whole fruits (Raffo et al., 2011; van den Berg et al., 2014). The effect of this processing and extraction process on the actual risk of the (herbal) tea and PFS samples 
should be investigated in more detail. In addition, the PA levels presented, and thus the calculated MOE values, are based on adding up the levels of all 1,2-unsaturated PAs and using the $\mathrm{BMDL}_{10}$ value of lasiocarpine. This might also contribute to an overestimation of the risk. The results of the present study show that PoD values for other PAs are 3 to 60 -fold higher than the $\mathrm{BMDL}_{10}$ of lasiocarpine. For this reason, calculated MOEs might overestimate the potential risk for human health. Close analysis of the type of PAs present in the different herbal teas and PFS revealed that in most cases lasiocarpine is not a major PA and in the majority of samples it is even absent. However, for the major PAs present in the samples, PoDs were absent hampering a further refinement of the MOE calculation. Whether the $\mathrm{BMDL}_{10}$ for lasiocarpine is really the most appropriate or rather a too conservative PoD for risk assessment remains to be established. Use of REP factors to take the relative potencies into account may be a way forward to. It is of interest to note that the interim REP factors defined by Merz and Schrenk (Merz and Schrenk, 2016) were based on data from in vitro cytotoxicity, genotoxicity in Drosophila, and acute toxicity in rodents (LD50), and did not take into account in vivo potencies for tumour formation. Comparison of the T10 values presented in the current study to the $\mathrm{BMDL}_{10}$ of lasiocarpine, provided REP factors for the relative potency in tumour formation of riddelline, monocrotaline, clivorine, senkirkine and symphytine of $0.39,0.05,0.23,0.03$, and 0.02 respectively, suggesting there is room for further refinement of the interim REP factors now available. This would likely further increase the MOE values.

Another factor to take into account is that the MOE calculations assume daily use of the teas and PFS during a whole lifetime. Calculation of the MOEs using the EDI values over a lifetime might overestimate the potential risk for human health and may therefore not be realistic. It could be considered that use of the (herbal) teas and PFS for only a few weeks instead of during a whole lifetime may decrease EDI values and increase MOE values by 2 to 3 orders of magnitude indicating their use would no longer be of concern. On the other hand some consumers may take more than one cup of tea a day and use the respective label for longer periods of time. Moreover, although as much as 28 PAs were monitored in the studies of Bodi et al. (2014), Mulder et al. (2015) and EFSA (2016), more PAs could be present in herbal teas and PFS that have eluded detection due to the lack of reference standards, resulting in underestimation of the PA content and consequently overestimation of the MOE values.

Yet another factor of importance to note is that the MOE will vary between different age groups. The present MOE values were calculated for adults while consumption of one cup of tea or $200 \mathrm{mg}$ of PFS by children with a 2-3 fold lower body weight will increase the EDI and decrease MOE values accordingly implying higher instead of lower concerns. The Committee On Toxicology (COT) already concluded that the age group with the highest PA exposure on a body weight basis would be infants (COT, 2008). In addition, the BfR concluded that there was a risk of health impairment, particularly in 
children (BfR, 2013).

With respect to the MOE values obtained for the PFS it is important to note that current EDI and resulting MOE values were based on an assumed daily intake of $200 \mathrm{mg}$ of supplement material per person per day. This amount is at the low end of intake estimates often recommended for botanical supplements on the respective labels (Van Den Berg et al., 2011). Intake of larger amounts would result in proportional increases of the EDI values and decreases of the MOE values resulting in values below 10000 if intake is much larger than $200 \mathrm{mg}$ a day for some of these PFS.

Finally, it should be considered that honey may be used by some consumers as a sweetener in their tea. Because honey is a food commodity that may also contain PAs (EFSA, 2011) this may further increase PA exposure upon tea consumption. The average level of contamination of retail honey was calculated for the sum of 8 PAs as $16 \mu \mathrm{g} / \mathrm{kg}$ for the lower bound and $26 \mu \mathrm{g} / \mathrm{kg}$ for the upper bound situation (EFSA, 2011). Data on what percentage of the tea drinkers would actually use honey as a sweetener are not available. One could estimate that using one spoon of 10 gram of honey per cup of tea on average would add between 0.16 and $0.26 \mu \mathrm{g}$ PAs to a cup of tea, amounting to between 0.00229 and $0.00371 \mu \mathrm{g} P \mathrm{Ps} / \mathrm{kg}$ bw for a $70 \mathrm{~kg}$ person. Using the $\mathrm{BMDL}_{10}$ of lasiocarpine of 0.07 $\mathrm{mg} / \mathrm{kg}$ bw/day this would result in an MOE between 18900 and 30600 and not be of concern as such, but in combination with (herbal) tea consumption this could in some cases result in MOEs lower than 10 000. In conclusion, use and also further refinement of REP factors defining the relative potency of PAs for tumour induction seems crucial for a refined MOE based risk assessment of products containing PAs. For (herbal) teas, the PA levels and resulting EDI and MOE values for chamomile flowers, peppermint, rooibos, black, green and mixed herbs, which are tea types derived from non-PAproducing plants, and for borago and coltsfoot teas which are derived from PA-producing plants, confirm the importance of risk management actions especially for consumers with regular daily use. For PFS, plant extract formula which were derived from PA- producing plants might pose a potential risk for human health. Our study provides additional insight in the current state-of-the art and limitations in risk assessment on PA-containing botanical food products, especially on (herbal) teas and PFS, indicating that PAs in food may present an important field of interest for current and future risk management.

\section{Acknowledgements}

This work was funded by a grant from the China Scholarship Council to Lu Chen (grant No.

201508650023). 


\section{References}

Benford, D., Bolger, P. M., Carthew, P., Coulet, M., DiNovi, M., Leblanc, J.-C., Renwick, A. G., Setzer, W., Schlatter, J., Smith, B., 2010. Application of the margin of exposure (MOE) approach to substances in food that are genotoxic and carcinogenic. Food Chem. Toxicol. 48, S2-S24.

BfR, 2009. Erste Einschätzung zur Bewertung der in raffinierten pflanzlichen Fetten nach-gewiesenen Gehalte von Glycidol-Fettsäureestern: Stellungnahme Nr. 007/2009 des BfR 10.

BfR, 2013. pyrrolizidine alkaloids in herbal teas and teas.

Bodi, D., Ronczka, S., Gottschalk, C., Behr, N., Skibba, A., Wagner, M., Lahrssen-Wiederholt, M., Preiss-Weigert, A., These, A., 2014. Determination of pyrrolizidine alkaloids in tea, herbal drugs and honey. Food Addit. Contam. : Part A. 31, 1886-1895.

COT, 2003. Safe Upper Levels for Vitamins and Minerals Expert Group on Vitamins and Minerals.

COT, 2008. COT Statement on Pyrrolizidine Alkaloids in Food.

ECHA, 2008. Guidance on information requirements and chemical safety assessment Chapter R.8: Characterisation of dose [concentration]-response for human health. 8 .

Edler, L., Hart, A., Greaves, P., Carthew, P., Coulet, M., Boobis, A., Williams, G. M., Smith, B., 2014. Selection of appropriate tumour data sets for Benchmark Dose Modelling (BMD) and derivation of a Margin of Exposure (MoE) for substances that are genotoxic and carcinogenic: Considerations of biological relevance of tumour type, data quality and uncertainty assessment. Food Chem. Toxicol. 70, 264-289.

EFSA, 2005. Opinion of the Scientific Committee on a Request from EFSA Related to a Harmonised Approach for Risk Assessment of Sub-stances which Are Both Genotoxic and Carcinogenic. EFSA J. 282, 1-31.

EFSA, 2011. Scientific Opinion on Pyrrolizidine alkaloids in food and feed. EFSA J. 9.

EFSA, 2012. Guidance on selected default values to be used by the EFSA Scientific Committee, Scientific Panels and Units in the absence of actual measured data. EFSA J. 10.

EFSA, 2016. Dietary exposure assessment to pyrrolizidine alkaloids in the European population. EFSA J. 14.

EFSA, 2016. Risks for human health related to the presence of 3- and 2-monochloropropanediol (MCPD), and their fatty acid esters, and glycidyl fatty acid esters in food. EFSA J. 14.

Griffin, C. T., Danaher, M., Elliott, C. T., Kennedy, D. G., Furey, A., 2013. Detection of pyrrolizidine alkaloids in commercial honey using liquid chromatography-ion trap mass spectrometry. Food Chem. 136, 1577-1583.

Hirono, I., Haga, M., Fujii, M., Matsuura, S., Matsubara, N., Nakayama, M., Furuya, T., Hikichi, M., Takanashi, H., Uchida, E., 1979. Induction of hepatic tumors in rats by senkirkine and symphytine. J. Natl. Cancer Inst. 63, 469-472.

IARC, 1976. IARC Monographs on Evaluation of Carcinogenic Risk of chemicals to Man: Some Naturally Occuring Substances. 10.

IARC, 1983. IARC Monographs on Evaluation of Carcinogenic Risk of chemicals to Humans: Some Food Additives, Feed Additives and Naturally Occurring Substances. 31.

IARC, 2002. IARC Monographs on Evaluation of Carcinogenic Risk of Chemicals to Humans: Some Traditional Herbal Medicines, Some Mycotoxins,Naphthalene and Styrene. 82.

IPCS, 1988. International Programme on Chemical Safety Health and Safety Environmental Health Criteria 80: Pyrrolizidine alkaloids.

Kuhara, K., Takanashi, H., Hirono, I., Furuya, T., Asada, Y., 1980. Carcinogenic activity of clivorine, a pyrrolizidine alkaloid isolated from Ligularia dentata. Cancer Lett. 10,117-122.

MacGregor, J. T., Frötschl, R., White, P. A., Crump, K. S., Eastmond, D. A., Fukushima, S., Guérard, M., Hayashi, M., Soeteman-Hernández, L. G., Johnson, G. E., Kasamatsu, T., Levy, D. D., Morita, T., Müller, L., Schoeny, R., Schuler, M. J., Thybaud, V., 2015. IWGT report on quantitative approaches to genotoxicity risk assessment II. Use of point-of-departure (PoD) metrics in defining acceptable exposure limits and assessing human risk. Mutation Research/Genetic Toxicology and Environmental Mutagenesis. 783, 66-78. 
Benford, D., Bolger, P. M., Carthew, P., Coulet, M., DiNovi, M., Leblanc, J.-C., Renwick, A. G., Setzer, W., Schlatter, J., Smith, B., 2010. Application of the margin of exposure (MOE) approach to substances in food that are genotoxic and carcinogenic. Food Chem. Toxicol. 48, S2-S24.

BfR, 2009. Erste Einschätzung zur Bewertung der in raffinierten pflanzlichen Fetten nach-gewiesenen Gehalte von Glycidol-Fettsäureestern: Stellungnahme Nr. 007/2009 des BfR 10.

$\mathrm{BfR}, 2013$. pyrrolizidine alkaloids in herbal teas and teas.

Bodi, D., Ronczka, S., Gottschalk, C., Behr, N., Skibba, A., Wagner, M., Lahrssen-Wiederholt, M., Preiss-Weigert, A., These, A., 2014. Determination of pyrrolizidine alkaloids in tea, herbal drugs and honey. Food Addit. Contam. : Part A. 31, 1886-1895.

COT, 2003. Safe Upper Levels for Vitamins and Minerals Expert Group on Vitamins and Minerals. COT, 2008. COT Statement on Pyrrolizidine Alkaloids in Food.

ECHA, 2008. Guidance on information requirements and chemical safety assessment Chapter R.8: Characterisation of dose [concentration]-response for human health. 8.

Edler, L., Hart, A., Greaves, P., Carthew, P., Coulet, M., Boobis, A., Williams, G. M., Smith, B., 2014. Selection of appropriate tumour data sets for Benchmark Dose Modelling (BMD) and derivation of a Margin of Exposure (MoE) for substances that are genotoxic and carcinogenic: Considerations of biological relevance of tumour type, data quality and uncertainty assessment. Food Chem. Toxicol. 70, 264-289.

EFSA, 2005. Opinion of the Scientific Committee on a Request from EFSA Related to a Harmonised Approach for Risk Assessment of Sub-stances which Are Both Genotoxic and Carcinogenic. EFSA J. 282, 1-31.

EFSA, 2011. Scientific Opinion on Pyrrolizidine alkaloids in food and feed. EFSA J. 9.

EFSA, 2012. Guidance on selected default values to be used by the EFSA Scientific Committee, Scientific Panels and Units in the absence of actual measured data. EFSA J. 10.

EFSA, 2016. Dietary exposure assessment to pyrrolizidine alkaloids in the European population. EFSA J. 14.

EFSA, 2016. Risks for human health related to the presence of 3- and 2-monochloropropanediol (MCPD), and their fatty acid esters, and glycidyl fatty acid esters in food. EFSA J. 14.

Griffin, C. T., Danaher, M., Elliott, C. T., Kennedy, D. G., Furey, A., 2013. Detection of pyrrolizidine alkaloids in commercial honey using liquid chromatography-ion trap mass spectrometry. Food Chem. 136, 1577-1583.

Hirono, I., Haga, M., Fujii, M., Matsuura, S., Matsubara, N., Nakayama, M., Furuya, T., Hikichi, M., Takanashi, H., Uchida, E., 1979. Induction of hepatic tumors in rats by senkirkine and symphytine. J. Natl. Cancer Inst. 63, 469-472.

IARC, 1976. IARC Monographs on Evaluation of Carcinogenic Risk of chemicals to Man: Some Naturally Occuring Substances. 10.

IARC, 1983. IARC Monographs on Evaluation of Carcinogenic Risk of chemicals to Humans: Some Food Additives, Feed Additives and Naturally Occurring Substances. 31.

IARC, 2002. IARC Monographs on Evaluation of Carcinogenic Risk of Chemicals to Humans: Some Traditional Herbal Medicines, Some Mycotoxins,Naphthalene and Styrene. 82.

IPCS, 1988. International Programme on Chemical Safety Health and Safety Environmental Health Criteria 80: Pyrrolizidine alkaloids.

Kuhara, K., Takanashi, H., Hirono, I., Furuya, T., Asada, Y., 1980. Carcinogenic activity of clivorine, a pyrrolizidine alkaloid isolated from Ligularia dentata. Cancer Lett. 10,117-122.

MacGregor, J. T., Frötschl, R., White, P. A., Crump, K. S., Eastmond, D. A., Fukushima, S., Guérard, M., Hayashi, M., Soeteman-Hernández, L. G., Johnson, G. E., Kasamatsu, T., Levy, D. D., Morita, T., Müller, L., Schoeny, R., Schuler, M. J., Thybaud, V., 2015. IWGT report on quantitative approaches to genotoxicity risk assessment II. Use of point-of-departure (PoD) metrics in defining acceptable exposure limits and assessing human risk. Mutation Research/Genetic Toxicology and Environmental Mutagenesis. 783, 66-78.

Merz, K.-H., Schrenk, D., 2016. Interim Relative Potency Factors for the Toxicological Risk Assessment of Pyrrolizidine Alkaloids in Food and Herbal Medicines. Toxicol. Lett. 263, 4457. 
Mori, H., Sugie, S., Yoshimi, N., Asada, Y., Furuya, T., Williams, G. M., 1985. Genotoxicity of a variety of pyrrolizidine alkaloids in the hepatocyte primary culture-DNA repair test using rat, mouse, and hamster hepatocytes. Cancer Res. 45, 3125-3129.

Mulder, P. P., Sánchez, P. L., These, A., Preiss-Weigert, A., 2015. Occurrence of Pyrrolizidine Alkaloids in food. EFSA Supporting Publications. 12.

NTP, 1978. Bioassay of lasiocarpine for possible carcinogenicity. Natl Toxicol Program Tech Rep Ser. $39,1$.

NTP, 2003. Toxicology and carcinogenesis studies of riddelliine (CAS No. 23246-96-0) in F344/N rats and B6C3F1 mice (gavage studies). Natl Toxicol Program Tech Rep Ser. 508.

Raffo, A., Nicoli, S., Leclercq, C., 2011. Quantification of estragole in fennel herbal teas: implications on the assessment of dietary exposure to estragole. Food Chem. Toxicol. 49, 370-375.

Shimshoni, J. A., Duebecke, A., Mulder, P. P., Cuneah, O., Barel, S., 2015. Pyrrolizidine and tropane alkaloids in teas and the herbal teas peppermint, rooibos and chamomile in the Israeli market. Food Addit Contam. 32, 2058-2067.

Shumaker, R., Robertson, K. A., Hsu, I., Allen, J., 1976. Neoplastic transformation in tissues of rats exposed to monocrotaline or dehydroretronecine. J. Natl. Cancer Inst. 56, 787-790.

Van Den Berg, S. J., Restani, P., Boersma, M. G., Delmulle, L., Rietjens, I. M., 2011. Levels of genotoxic and carcinogenic compounds in plant food supplements and associated risk assessment. Food Nutr Sci. 2, 989.

Van den Berg, S. J. P. L., Alhusainy, W., Restani, P., Rietjens, I. M. C. M., 2014. Chemical analysis of estragole in fennel based teas and associated safety assessment using the Margin of Exposure (MOE) approach. Food Chem. Toxicol. 65, 147-154. 


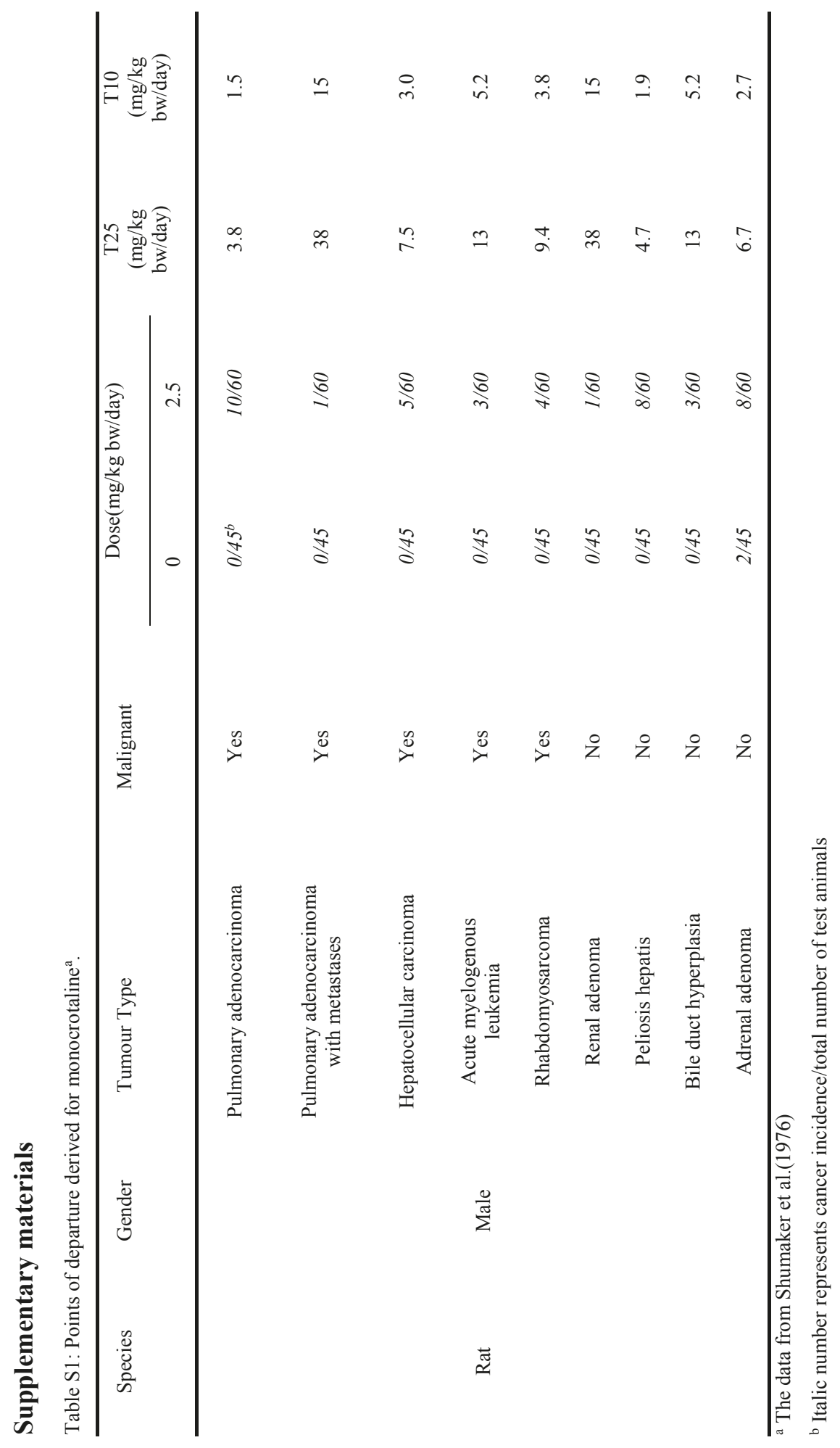




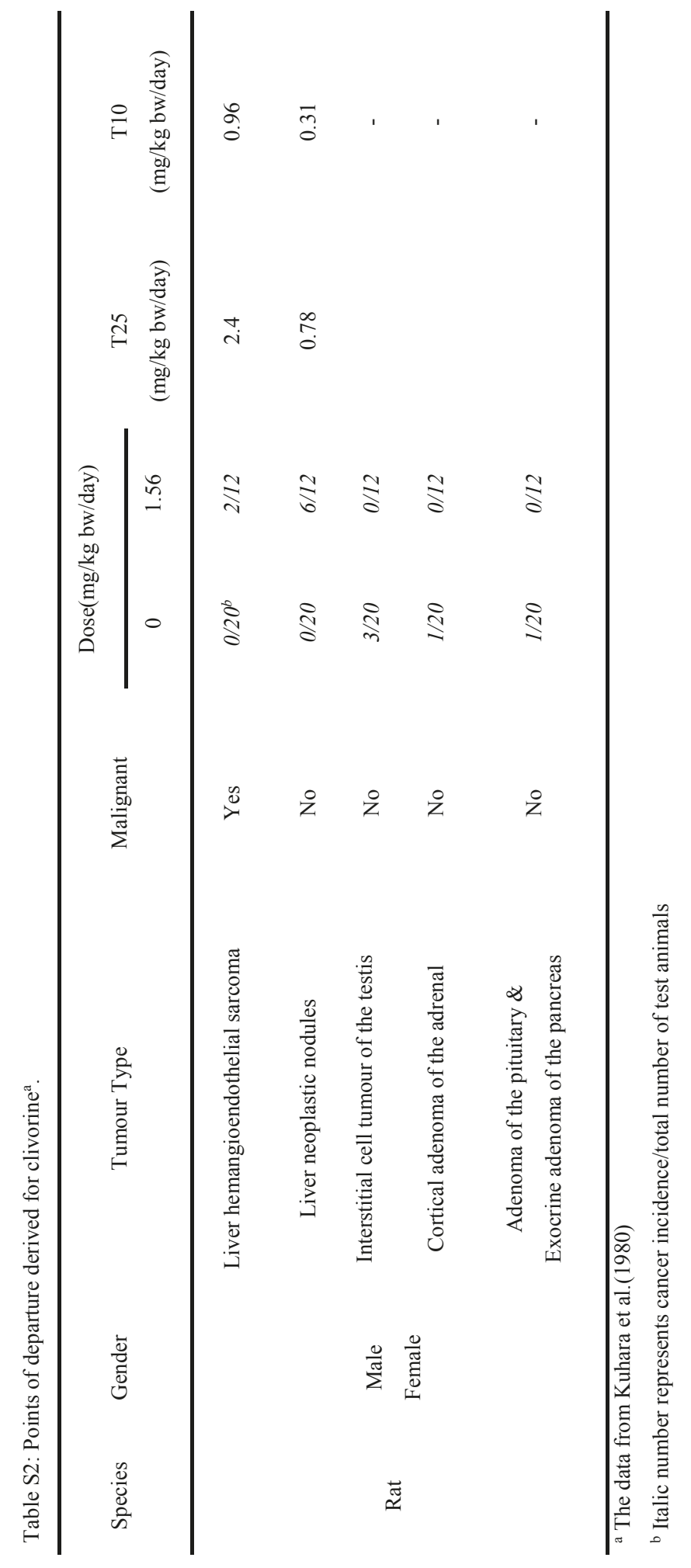




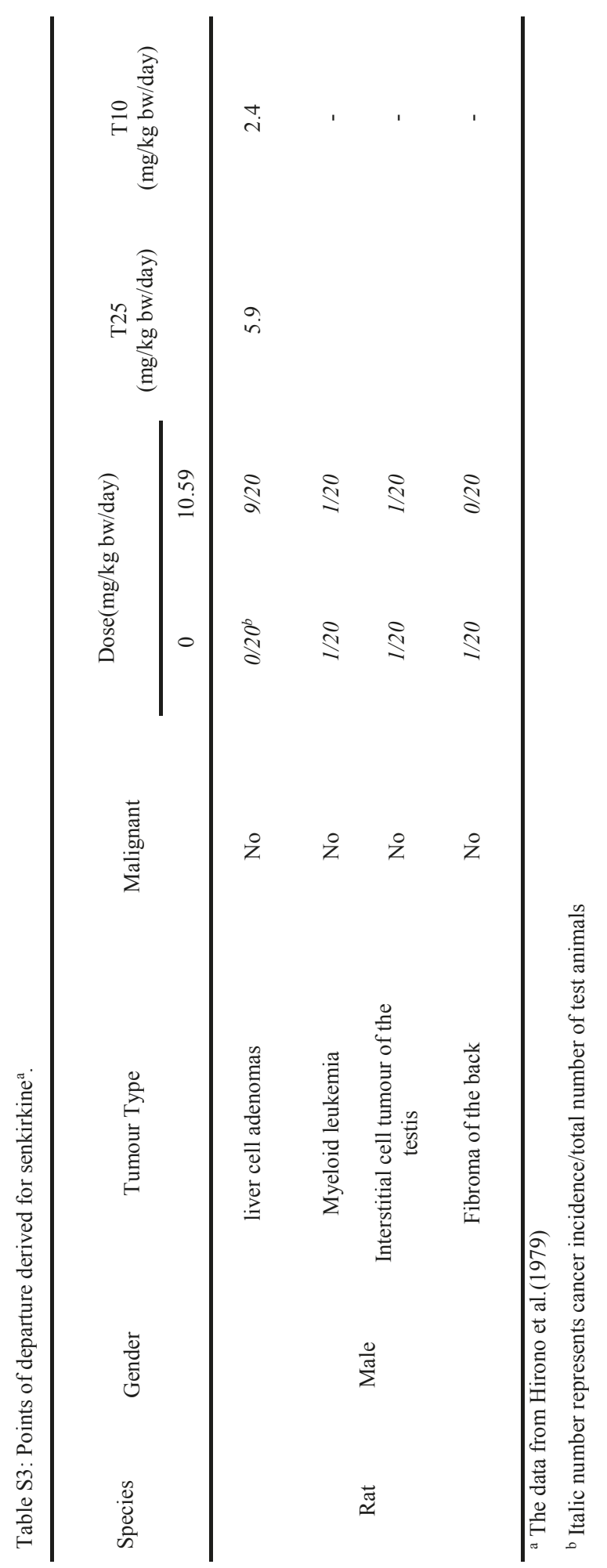

53 


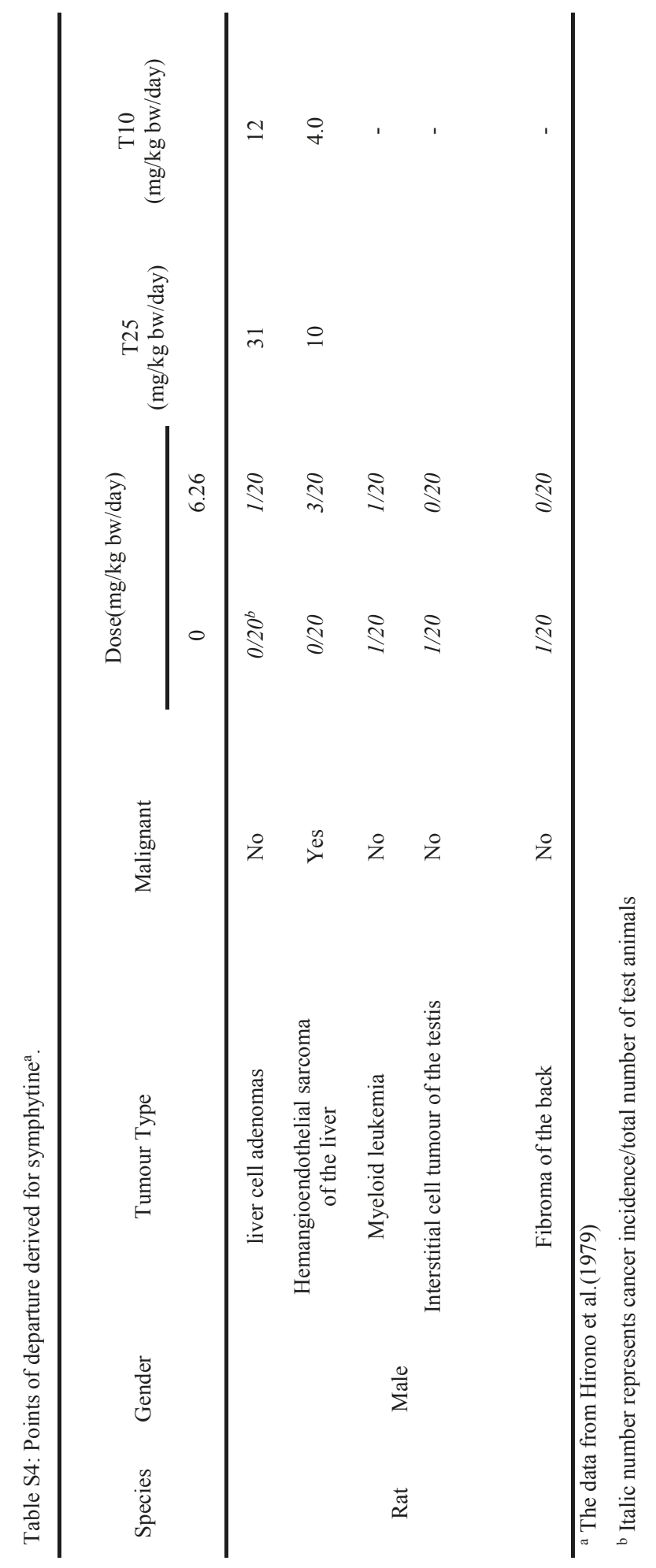




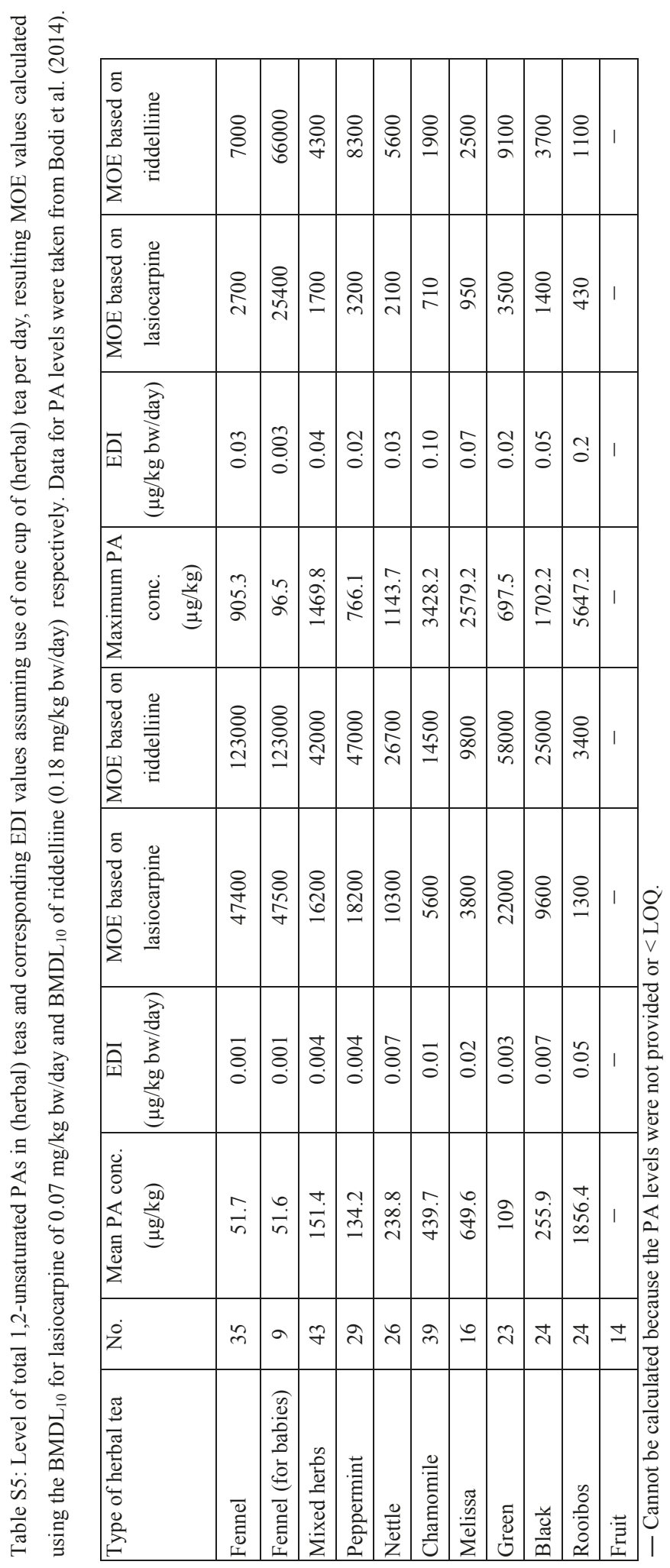




\begin{tabular}{|c|c|c|c|c|c|c|c|c|c|c|}
\hline 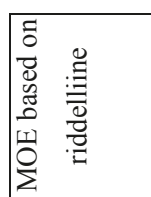 & 㤂 & 1 & 1 & $\stackrel{8}{\Xi}$ & 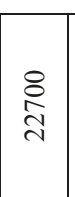 & : & ષ્ఠ & $\underset{\infty}{\stackrel{ి}{~}}$ & 1 & 1 \\
\hline 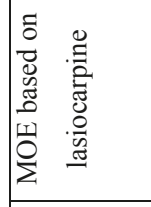 & 荢 & 1 & 1 & 足 & $\mid \begin{array}{c}0 \\
\infty \\
\infty \\
\infty\end{array}$ & 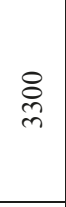 & ఫ్లి & 今్ల & 1 & 1 \\
\hline 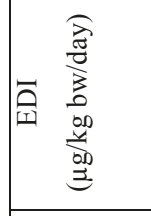 & $\bar{o}_{0}$ & 1 & 1 & $\stackrel{0}{0}$ & $\mid$ & $\begin{array}{l}0 \\
0 \\
0\end{array}$ & $\stackrel{8}{0}$ & $\begin{array}{l}0 \\
0 \\
0\end{array}$ & 1 & 1 \\
\hline 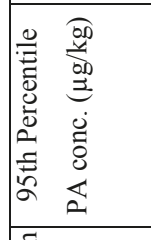 & 角 & 1 & 1 & in & $\begin{array}{l}\stackrel{n}{a} \\
\stackrel{n}{a}\end{array}$ & $\stackrel{m}{\sim}$ & : & $\mid \begin{array}{c}n \\
\substack{1 \\
N}\end{array}$ & 1 & 1 \\
\hline 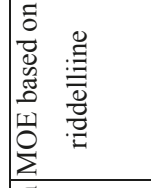 & \begin{tabular}{|l|} 
\\
8 \\
$b$ \\
$\dot{b}$
\end{tabular} & 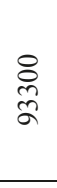 & $\begin{array}{l}\text { ఫे } \\
\text { ఫु }\end{array}$ & 品 & $\begin{array}{l}\text { 总 } \\
\stackrel{0}{\circ}\end{array}$ & $\begin{array}{l}8 \\
8 \\
0 \\
0\end{array}$ & 亲 & 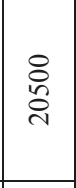 & 高 & $\begin{array}{l}8 \\
8 \\
\text { a }\end{array}$ \\
\hline 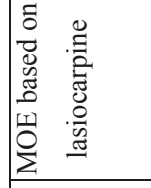 & $\begin{array}{l}8 \\
\frac{8}{\infty} \\
\sim\end{array}$ & $\begin{array}{l}\stackrel{8}{0} \\
\text { de }\end{array}$ & 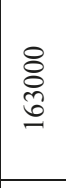 & $\mid$\begin{tabular}{l}
8 \\
+ \\
\multirow{2}{*}{}
\end{tabular} & 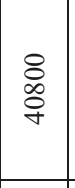 & 寻 & ০০ & 灾 & 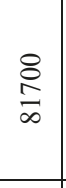 & 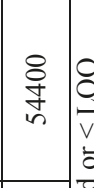 \\
\hline 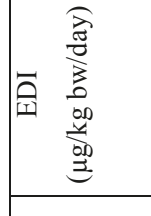 & 蒿 & $\tilde{\sigma}_{0}$ & 善 & $\mid \begin{array}{c}0 \\
0 \\
0 \\
0\end{array}$ & $\tilde{\Xi}$ & 菅 & 文 & 容 & 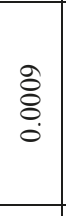 & $\overrightarrow{0}$ \\
\hline 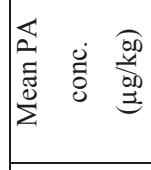 & $\cong$ & $\frac{n}{6}$ & $\cong$ & $\AA$ & 8 & 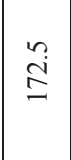 & तु̀ं & 盀 & 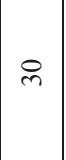 & 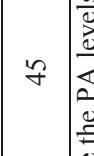 \\
\hline$\dot{z}$ & $\tilde{\Xi}$ & \pm & $\simeq$ & ले & $\stackrel{\circ}{m}$ & $\stackrel{\circ}{a}$ & 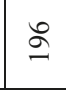 & $\Xi$ & $\stackrel{\square}{\square}$ & के \\
\hline 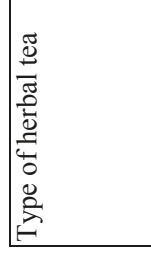 & 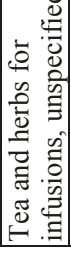 & & 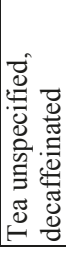 & & $\bar{\Xi}$ & 产 & 童 & 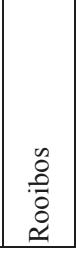 & 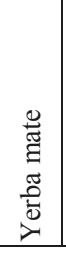 & 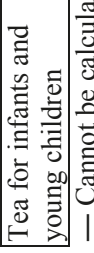 \\
\hline
\end{tabular}




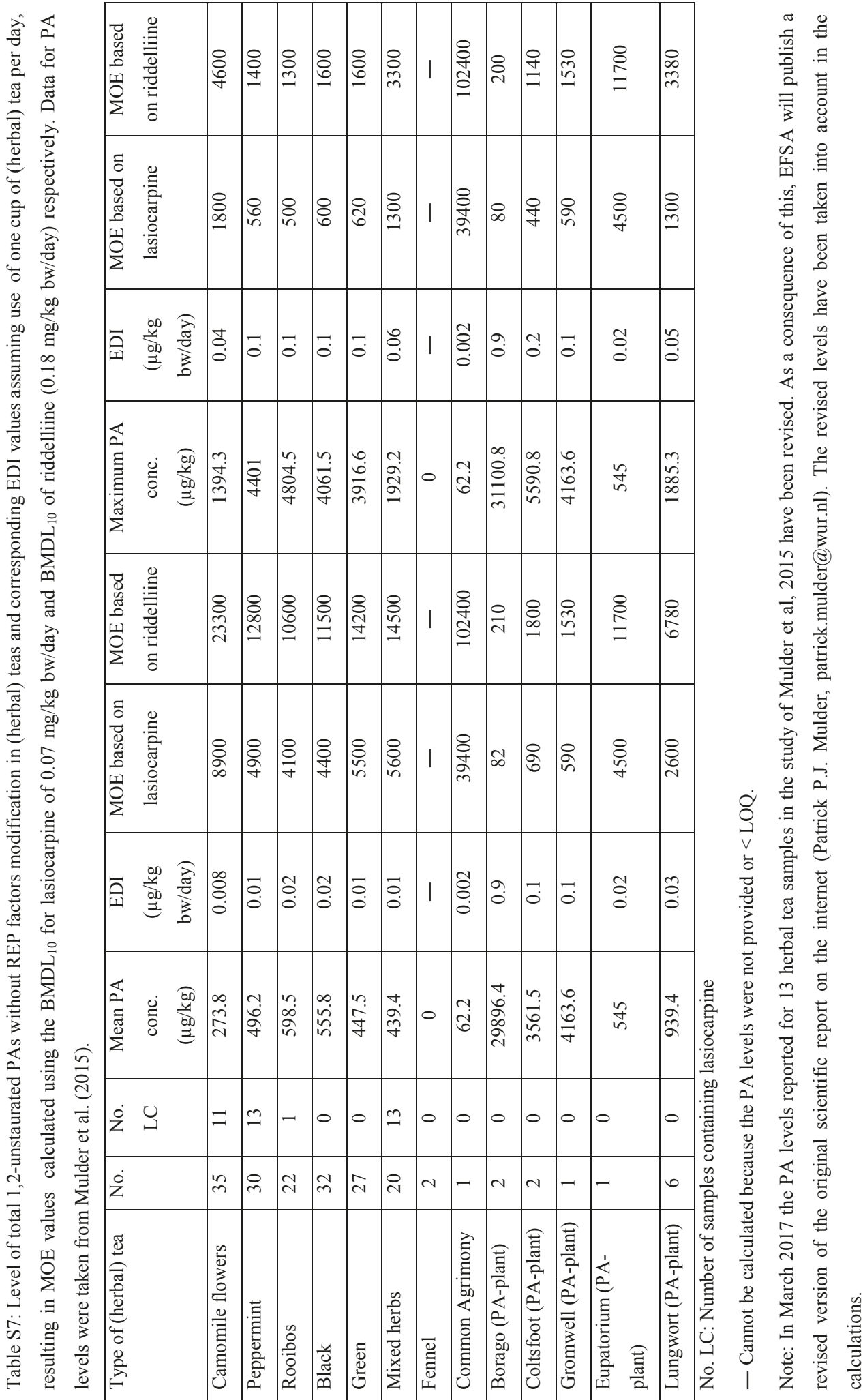




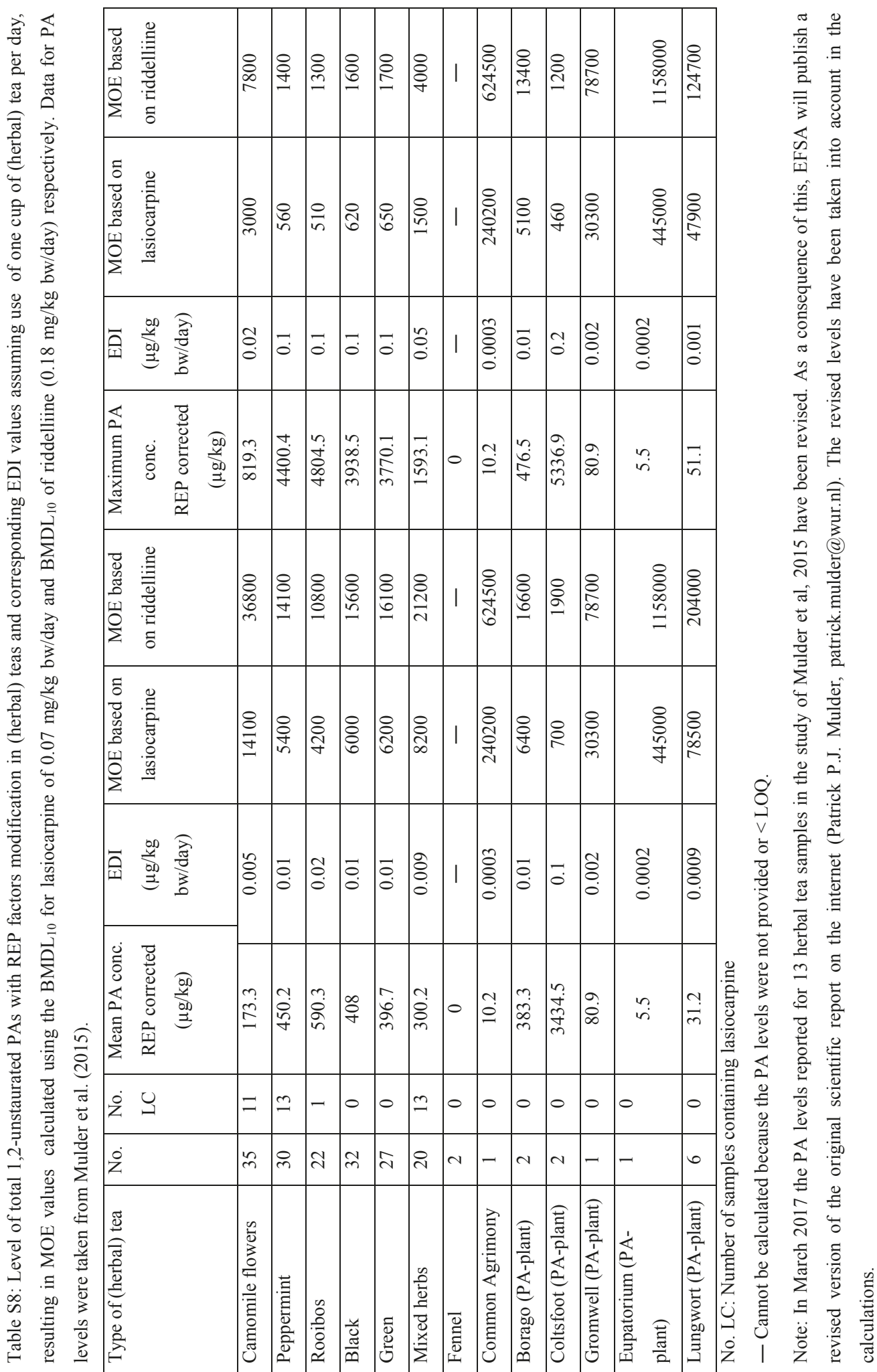




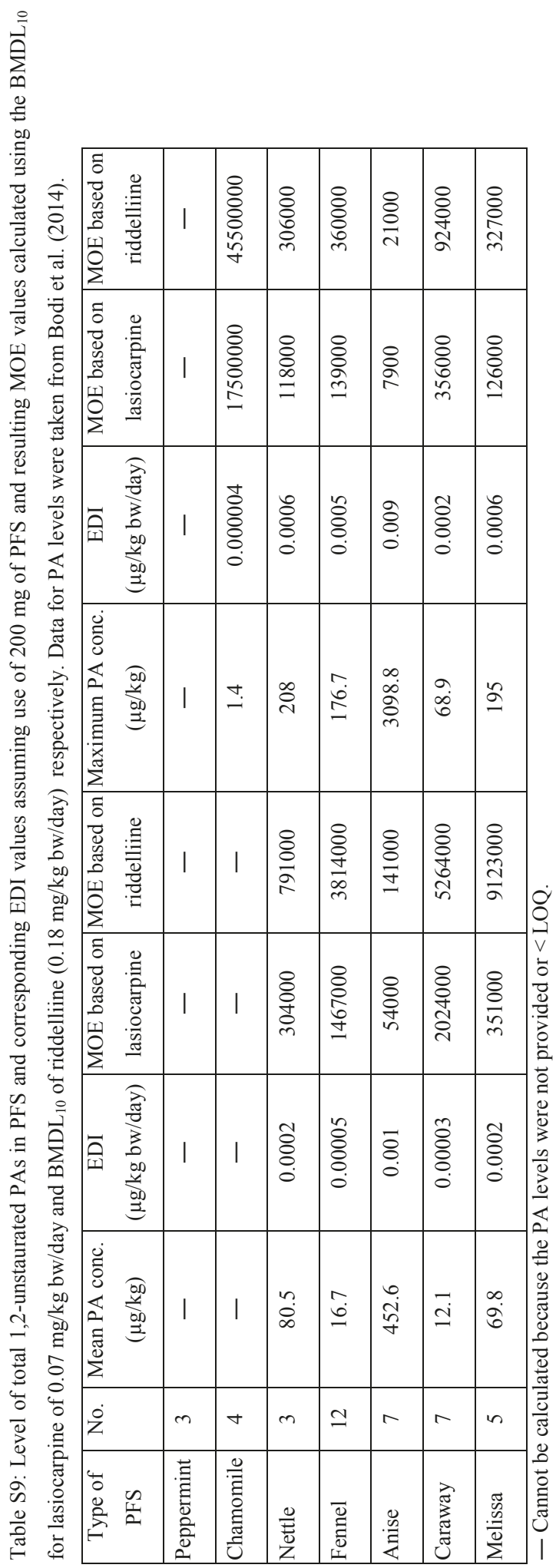




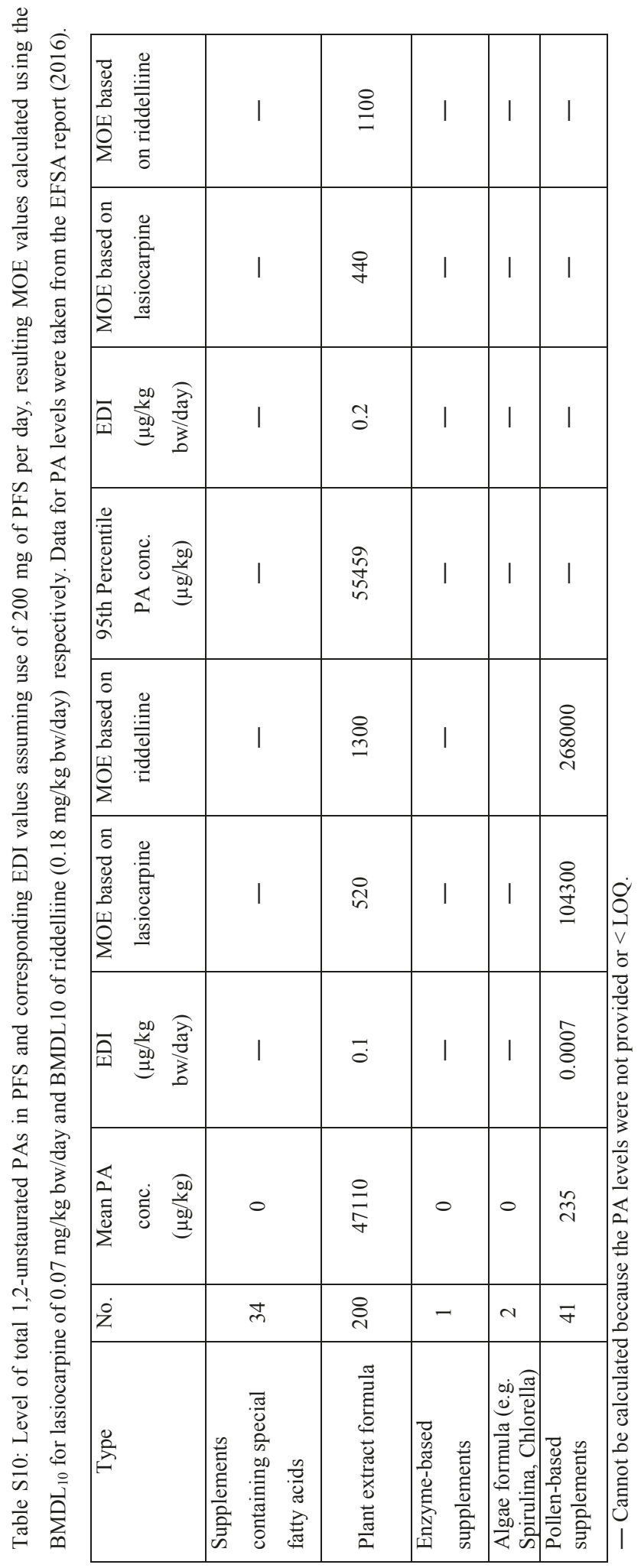




\section{Chapter 3}

\section{Risk assessment of intake of pyrrolizidine alkaloids from herbal teas and medicines following realistic exposure scenarios}

Lu Chen, Patrick P.J. Mulder, Ad Peijnenburg, Ivonne M.C.M. Rietjens

Published in: Food and Chemical Toxicology, (2019) 130, 142-153 


\begin{abstract}
In this study five types of herbal teas were used to quantify the effect of comminution of the leaves on resulting PA exposure. Results show that PA levels extracted from intact leaves were consistently lower than from comminuted tea leaves. The Margin of Exposure (MOE) approach was applied to evaluate the consequences of this difference for the associated risks in the scenario of lifetime exposure. Furthermore, we considered medicinal use of these teas for shorter-than-lifetime exposure scenarios, and also analysed the risks of shorter-than-lifetime use of eight herbal medicines and 19 previously analysed plant food supplements. This analysis revealed that shorter-than-lifetime use resulted in MOE values < 10,000 upon use for 40 to 3450 weeks during a lifetime, with for only a limited number of herbal teas and medicines use of two weeks a year (150 weeks during a 75 year lifetime) would still raise a concern. It is concluded that taking more realistic conditions into account markedly reduces the concerns raised for these herbal preparations. These results also illustrate the need for development of a generally accepted method for taking short term exposure into account in risk assessment of compounds that are genotoxic and carcinogenic.
\end{abstract}




\section{Introduction}

Botanicals and botanical preparations, such as herbal teas and herbal medicines, have been marketed for several decades. Due to (misleading) advertisement and/or overstatement of the benefits arising from consumption of these products and also because many customers equate 'natural' with 'safe', botanical preparations are widely used around the world (Rietjens et al., 2008). In addition to the fact that benefits of these preparations are often not scientifically proven and/or due to illegal adulteration with pharmaceutical ingredients (Ancuceanu et al., 2013; Carvalho et al., 2012; Reeuwijk et al., 2014), botanical preparations may even contain ingredients of concern. For example, herbal teas and plant food supplements (PFS) have been shown to frequently contain toxic pyrrolizidine alkaloids (PAs) (Bodi et al., 2014; EFSA, 2016; Mulder et al., 2018; Mulder et al., 2015). Especially 1,2-unsaturated PAs have been shown to be hepatotoxic, genotoxic and carcinogenic in rats and other experimental rodents (Hirono et al., 1979; Hirono et al., 1976; Kuhara et al., 1980; NTP, 1978, 2003; Schoental, 1970; Shumaker et al., 1976). These results also raise a concern for human health when levels of intake would be too high. A number of cases of intoxication and even death caused by PAs through consumption of herbal teas and herbal medicines have been reported (Lin et al., 2011; Margalith et al., 1985; Mohabbat et al., 1976; Ruan et al., 2015; Tandon et al., 1976; Weston et al., 1987). Intake of herbal teas and medicines has proven to be a major route for human exposure to PA-containing plants (Bodi et al., 2014; Edgar et al., 1992; Roeder, 2000).

In light of the health relevance of PAs derived from herbal teas, recently a number of studies have performed a risk assessment for PAs in herbal teas (BfR, 2013; Chen et al., 2017; EFSA, 2016). Given that 1,2-unsaturated PAs are genotoxic and carcinogenic, this risk assessment was based on the Margin of Exposure (MOE) approach. An MOE cut-off value of 10,000 is generally applied, which incorporates factors including possible inter-species and intra-species differences in toxicokinetics and toxicodynamics, inter-individual human variability in cell cycle control and DNA repair, and the fact that the $\mathrm{BMDL}_{10}$ (the lower confidence limit of the dose level that results in $10 \%$ extra cancer incidence above background level), is not a no effect level (EFSA, 2005). MOE values below 10,000 indicate that there might be a potential concern for human health (EFSA, 2005). The risk assessments suggested that long-term consumption of certain herbal teas may pose a potential risk for human health, especially when considering lifetime exposure (BfR, 2013; Chen et al., 2017; EFSA, 2016). These studies evaluated the MOE values for lifelong daily use of herbal teas based on occurrence data obtained from extraction of finely ground, comminuted leaves. Considering the fact that consumption of teas usually occurs by hot water extraction of partially intact or coarsely ground leaves, extraction of comminuted leaves may represent a worst case, and not reflect a real life scenario. This is especially of importance given that previous studies demonstrated that hot water extraction of alkenylbenzenes from comminuted fennel fruits is more efficient than that from the whole fruits (Raffo et al., 2011; van den Berg et al., 2014). It is conceivable that also for PAs use of comminuted leaves may facilitate their 
extraction from the teas. This may influence the actual exposure and corresponding risk assessment. Therefore, the aim of the present study was to compare the extraction of PAs from comminuted leaves and intact leaves of herbal teas, and quantify the consequences of possible differences for the MOE based risk assessment. Furthermore, when considering realistic exposure scenario's it should also be taken into account that herbal teas are frequently used for medicinal purposes, a practice being quite popular in many developing and developed countries (Fu et al., 2002; Prakash et al., 1999; Roeder, 2000; Stegelmeier et al., 1999). Thus, people might consume herbal teas as a medicine only during certain periods of for example illness. This shorter-than-lifetime use also holds true for herbal medicinal supplements, while evaluation by the MOE approach generally assumes lifelong everyday use. According to a survey of usage of herbal preparations by European adults, the majority of all respondents indicated to take these botanicals only during a specified time period or when they had a worsened condition (Garcia-Alvarez et al., 2014). In addition shorter-than-lifetime use of these herbal preparations is in line with the EMA (European Medicines Agency) who indicates for example that bitter fennel preparations should not be taken for periods exceeding two weeks (EMA, 2008). The limit of two weeks was established by EMA considering the lack of available safety data on long term exposure and the traditional short term use of such herbal products in self-medication (EMA, 2007). The German and Netherlands regulations indicate that the use of PA containing preparations should be limited to short time (defined as 6 weeks) use at dose levels of $1 \mu \mathrm{g}$ /day (Bundesgesundheitsamt, 1992; WKB, 2001). It is thus of significance to analyse the presence of PAs in extracts of comminuted and whole leaf teas and herbal medicines and perform a risk assessment taking shorter exposure duration into consideration. In our previous study, we used the data from Bodi et al. (2014), Mulder et al. (2015) and EFSA (2016) to perform a risk assessment for herbal teas and PFS based on daily consumption of one cup of tea or $200 \mathrm{mg}$ of PFS per day during a lifetime, mainly because lifetime exposure is the default assumption in the MOE approach (Chen et al., 2017). This evaluation confirmed that consumption of certain herbal teas and PFS may raise a health concern when consumed regularly during a lifespan (Chen et al., 2017). The aim of the present study was to assess the risk of exposure to PAs from herbal teas, herbal medicines and PFS using the MOE approach considering shorter and more realistic exposure scenarios, also taking the potentially lower extraction efficiency from whole herbal tea leaves versus comminuted samples into account.

\section{Materials and Methods}

\section{Standards and reagents}

Formic acid (analytical grade) and ammonium carbonate (analytical grade) were obtained from SigmaAldrich, Zwijndrecht, the Netherlands). Acetonitrile (LC-MS grade) and methanol (LC-MS grade) were obtained from Actu-all, Oss, the Netherlands). Fifty-four PA analytical standards were sourced from Phytoplan (Heidelberg, Germany), except for: heliotrine and trichodesmine from Latoxan (Valence, France); usaramine from BOC Sciences (Shirley, NY, USA), florosenine from PRISNA 
(Leiden, the Netherlands), echimidine, indicine, indicine $\mathrm{N}$-oxide, intermedine, intermedine $\mathrm{N}$-oxide, lycopsamine, lycopsamine N-oxide, monocrotaline, monocrotaline N-oxide and otosenine from Phytolab (Vestenbergsgreuth, Germany). Usaramine N-oxide, trichodesmine N-oxide were in-house synthesized by the method of (Chou et al., 2003). See Supplementary Data 1 for a full list of PA standards used in this study.

Stock solutions of the 54 available PAs were prepared in methanol $(100 \mu \mathrm{g} / \mathrm{mL})$. A mixed solution (1 $\mu \mathrm{g} / \mathrm{mL}$ in methanol) containing all PA standards was prepared from the stock solutions. This mixed standard solution was used to spike the herbal teas and medicine samples as described below.

\section{Herbal teas and PA extraction}

Five types of herbal teas derived from PA-producing plants were used to investigate the difference of PAs extraction efficiency between intact leaves and comminuted leaves, including coltsfoot (Tussilago farfara), comfrey (Symphytum officinale), borage (Borago officinalis), climbing groundsel (Senecio scandens) and sunn hemp (Crotalaria juncea) teas. These herbal teas were selected, because they are available on the market as intact leaves. Table 1 presents an overview of the herbal teas used in the present study, their country and year of origin, and also the presumptive health effects of the respective teas as derived from the literatures.

The intact leaves of each tea were randomly selected and ground to produce the comminuted leaves using a grinder (HR2056, Philips, the Netherlands). The intact leaves as well as the comminuted leaves of these teas were used for hot water extraction and subsequent PA analysis. For PA extraction, $2 \mathrm{~g}$ of the sample were positioned in a $250 \mathrm{~mL}$ glass beaker and $150 \mathrm{~mL}$ of boiling water was poured onto the tea. The infusion was stirred 3 times in 10 minutes. This procedure was selected as a worst case scenario for extraction of bioactive ingredients upon hot water extraction based on literature (Molan et al., 2009; McKAY et al., 1995; Raffo et al., 2011). Then, the infusion passed through a paper filter. The extraction was performed in triplicate.

From each of the filtered infusions four aliquots were taken and transferred to autosampler vials. Of tea 1 (coltsfoot) aliquots of $400 \mu \mathrm{L}$; of tea 3 (borage) aliquots of $20 \mu \mathrm{L}$; of tea 2 (comfrey) and tea 4 (climbing groundsel) aliquots of $100 \mu \mathrm{L}$ and of tea 5 (sunn hemp) aliquots of $200 \mu \mathrm{L}$ were taken. For each infusion one of the aliquots was spiked with $25 \mathrm{ng} / \mathrm{mL}(25 \mu 1$ of $1 \mu \mathrm{g} / \mathrm{mL}$ PA mix) and one was spiked with $100 \mathrm{ng} / \mathrm{mL}$ ( $100 \mu \mathrm{L}$ of $1 \mu \mathrm{g} / \mathrm{mL}$ PA mix). The total volumes were made up to $1 \mathrm{~mL}$ with water. 


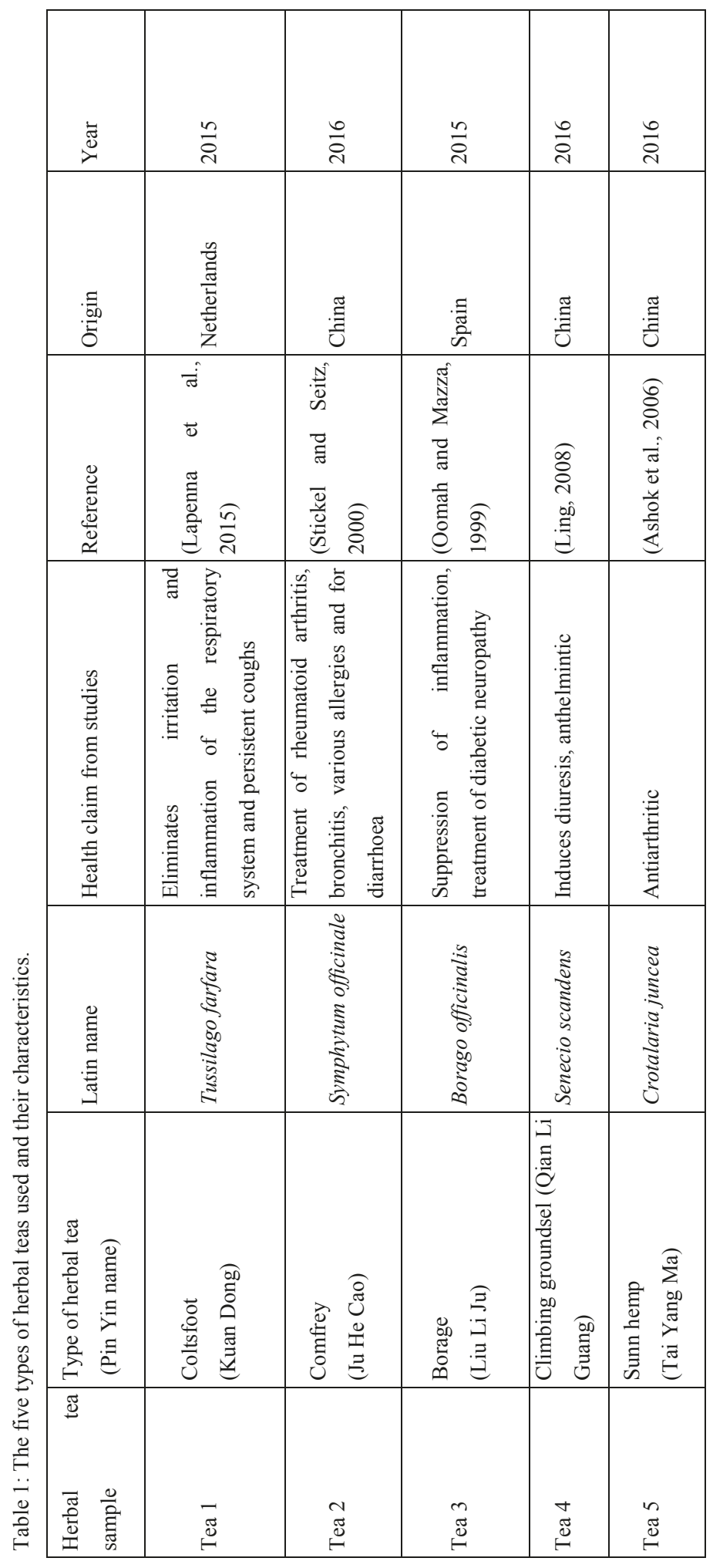




\section{Herbal medicines and PA extraction}

A total of eight herbal medicines were purchased from the Chinese market. The products were in the form of capsules, pills or tablets, four of them containing PA-producing plants and four containing non-PA-producing plants. Table 2 presents an overview of the collected herbal medicine samples and their characteristics.

For PA analysis from each sample three test portions of 1.0 gram were extracted with $20 \mathrm{~mL}$ of $1.0 \%$ formic acid solution by agitation for 30 minutes. Before extraction one of the test portions was fortified with the mixed PA standard solution at $500 \mu \mathrm{g} / \mathrm{kg}(500 \mu \mathrm{L}$ of $1 \mu \mathrm{g} / \mathrm{mL}$ PA mix). After centrifugation $5 \mathrm{~mL}$ of supernatant was transferred to a new tube and brought to $\mathrm{pH}$ 6-7 with $1 \mathrm{M}$ ammonium carbonate solution, $\mathrm{pH}$ 9. The extracts were further purified by SPE over a StrataX $200 \mathrm{mg}$, $6 \mathrm{cc}$ cartridge. Cartridges were conditioned with $6 \mathrm{~mL}$ methanol, followed by $6 \mathrm{~mL}$ water. After the extract was passed through the cartridge, this was washed with $6 \mathrm{~mL} \mathrm{1 \%}$ formic acid, followed by 6 $\mathrm{mL}$ water and dried under vacuum using a vacuum manifold for $10 \mathrm{~min}$. PAs were eluted with $6 \mathrm{~mL}$ of methanol and the eluates were dried under a stream of nitrogen at $50^{\circ} \mathrm{C}$ using a TurboVap. The extracts were reconstituted in $500 \mu \mathrm{L}$ of $10 \%$ methanol and filtered using $0.45 \mu \mathrm{m}$ PTFE filtervials (UniPrep, Whatman, Maidstone, UK).

Medicines 5 and 8 contained senkirkine at concentrations exceeding the spiked concentration of 500 $\mu \mathrm{g} / \mathrm{kg}$. Senkirkine was quantified in these samples by spiking aliquots $(1 \mathrm{~mL})$ of the herbal extracts with senkirkine at $50 \mu \mathrm{g} / \mathrm{mL}(5 \mu \mathrm{L}$ of a $10 \mu \mathrm{g} / \mathrm{mL}$ solution of senkirkine in methanol and at 250 $\mathrm{ng} / \mathrm{mL}$ ( $25 \mu \mathrm{L}$ of a $10 \mu \mathrm{g} / \mathrm{mL}$ solution of senkirkine). The fortifications correspond to 1000 and 5000 $\mu \mathrm{g} / \mathrm{kg}$ in the herbal medicine, respectively. 


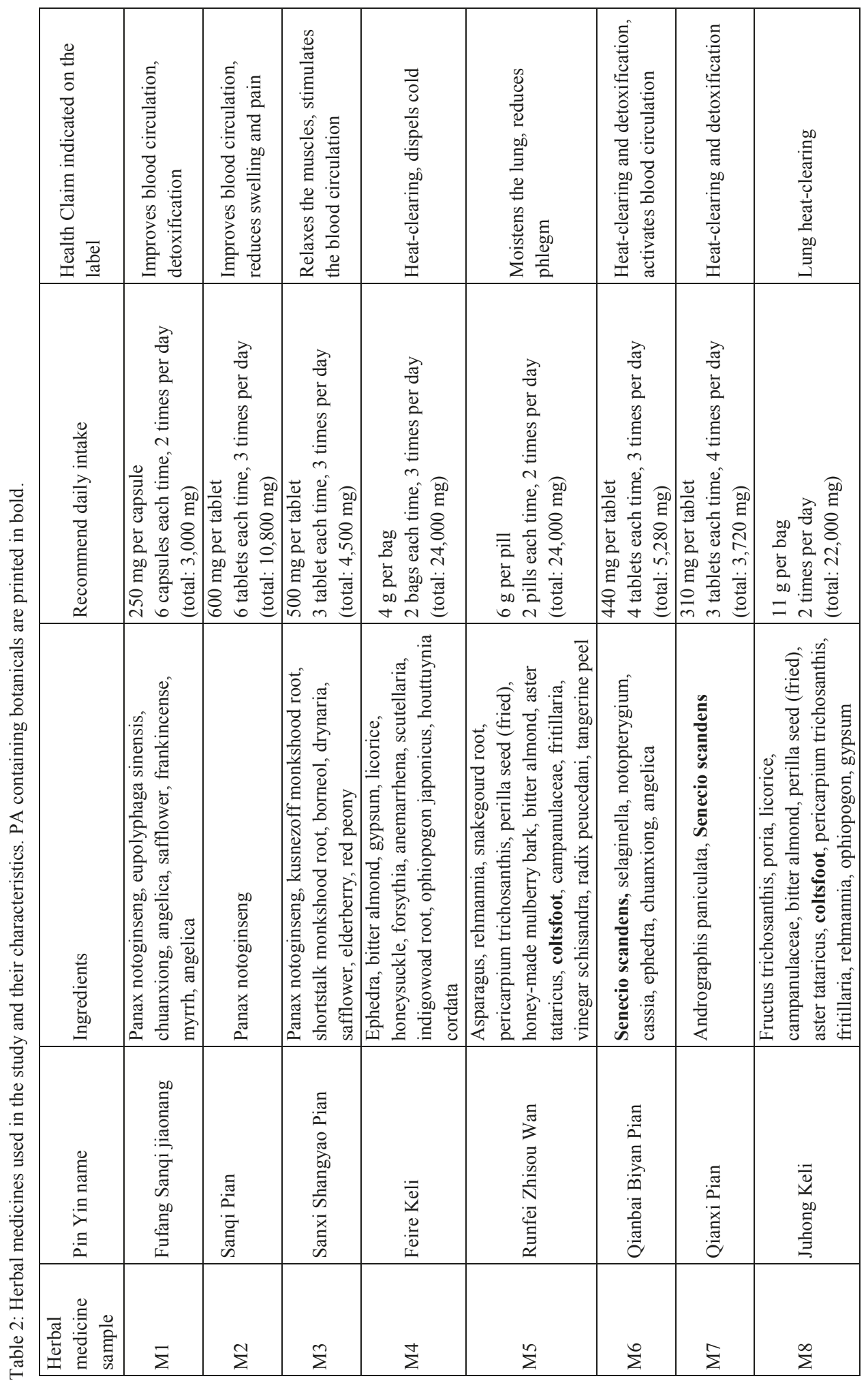




\section{LOD, LOQ, recovery, and precision data}

In-house validated methods for 54 PAs in herbal tea infusions and in PFS were used. For PAs in herbal infusions the LOQs obtained were at $0.05 \mu \mathrm{g} / \mathrm{L}$ and in herbal supplements at $4-5 \mu \mathrm{g} / \mathrm{kg}$. LODs were estimated at $0.01-0.02 \mu \mathrm{g} / \mathrm{L}$ in herbal infusions and at $1-2 \mu \mathrm{g} / \mathrm{kg}$ in PFS. For PFS recoveries (level: $100 \mu \mathrm{g} / \mathrm{kg}$ ) varied from $73-107 \%$. Repeatability $(\mathrm{n}=5$ ) ranged between 8.1 and $24 \%$ at 10 $\mu \mathrm{g} / \mathrm{kg}$, between 3.1 and $8.5 \%$ at $100 \mu \mathrm{g} / \mathrm{kg}$ and between 3.1 and $10.9 \%$ at $250 \mu \mathrm{g} / \mathrm{kg}$. Linearity of the LC-MS/MS system was checked by analysis of 8-point calibration curves prepared in blank tea extract and in blank PFS extract over the range of 0 to $250 \mathrm{ng} / \mathrm{mL}$.

\section{LC-MS/MS analysis}

Analysis of PAs was performed in positive electrospray mode on an LC-MS/MS system consisting of a Waters Acquity UPLC coupled to a Xevo TQ-S tandem mass spectrometer (Waters, Milford, MA, USA). At least two MRM transitions were measured per analyte. Besides the 54 PAs for which an analytical standard was available, the samples were screened for another 35 1,2-unsaturated PAs for which no standards were available. These PAs could be included in the analytical method because mass spectrometric data were available from the analysis of extracts of authentic Borago, Symphytum, Crotalaria, Senecio, Petasites and Tussilago plant samples. See Supplementary Data 1 for an overview of the MS/MS transitions used for the complete set of PAs. Chromatographic separation was obtained on a $150 \times 2.1 \mathrm{~mm}, 1.7 \mu \mathrm{m}$ particle size, UPLC BEH C18 analytical column (Waters, Milford, MA, USA). Eluent A consisted of water containing $10 \mathrm{mM}$ ammonium carbonate $\mathrm{pH} 9$ and acetonitrile was used as eluent $\mathrm{B}$. A gradient elution was performed as follows: $0.0 \mathrm{~min} 100 \% \mathrm{~A} / 0 \% \mathrm{~B}$, $0.1 \min 95 \% \mathrm{~A} / 5 \% \mathrm{~B}, 3.0 \min 90 \% \mathrm{~A} / 10 \% \mathrm{~B}, 7.0 \min 76 \% \mathrm{~A} / 24 \% \mathrm{~B}, 9.0 \min 70 \% \mathrm{~A} / 30 \% \mathrm{~B}, 12.0$ $\min 30 \% \mathrm{~A} / 70 \% \mathrm{~B}, 12.1-15.0 \min 100 \% \mathrm{~A} / 0 \% \mathrm{~B}$. The column was kept at $50^{\circ} \mathrm{C}$ and a flow rate of 400 $\mu \mathrm{L} /$ min was applied; $2 \mu \mathrm{L}$ sample extract was injected. For some PA isomers, e.g. lycopsamine and intermedine and their N-oxide and 7-acetyl analogues no or only partial separation could be obtained under the chromatographic conditions used. For verification of the identity of the isomers, tea samples 2 (Comfrey) and 3 (Borage) were reanalysed using acidic chromatography, which allows the separation of lycopsamine and intermedine isomers (Bodi et al., 2014). Comfrey tea contained intermedine and lycosamine isomers in equal amounts, while borage tea contained only the lycopsamine isomer (data not shown).

\section{Quantification of PAs in herbal tea infusions and in PFS}

Quantification of the PAs in the herbal infusions was based on one-point standard addition of PAs to the infusion. Depending on the concentration found in the extract, either the standard addition at 25 $\mathrm{ng} / \mathrm{mL}$ was used or the one at $100 \mathrm{ng} / \mathrm{mL}$.

The PAs in the herbal medicines were quantified based on one-point standard addition of $500 \mu \mathrm{g} / \mathrm{kg}$ to the herbal product. Medicines 5 and 8 contained senkirkine at higher concentrations and these were 
quantified by spiking the herbal extracts with senkirkine at 50 and $250 \mathrm{ng} / \mathrm{mL}$ (corresponding to 1000 and $5000 \mu \mathrm{g} / \mathrm{kg}$ in the herbal medicine, respectively).

PAs for which no PA reference standard was available were semi-quantified by taking a structurally related PA reference standard, as indicated in the Supplementary Data 1.

\section{Estimation of daily intakes of PAs resulting from the consumption of herbal teas and herbal medicines}

To perform a risk assessment, the daily PA intake resulting from use of the herbal teas was estimated assuming daily consumption of the amount of PAs extracted by hot water extraction from $2 \mathrm{~g}$ tea, corresponding to one cup of tea, as described before (BfR, 2013). For estimation of the PA exposure resulting from herbal medicines, the concentration of the PAs quantified in the extracts was multiplied by the daily use of the herbal medicines as recommended by the supplier (Table 2). The estimated daily intake (EDI) values were calculated using a default body weight of $70 \mathrm{~kg}$ for an adult as proposed by EFSA (EFSA, 2012).

$$
E D I=\frac{\text { total } P A \text { concentration } * \text { daily use }}{b w(70 \mathrm{~kg})}
$$

\section{Calculation of the Margin of Exposure (MOE)}

Risk assessment was performed using the MOE approach. EFSA has recently set a reference point of $237 \mu \mathrm{g} / \mathrm{kg}$ bw/day for riddelliine as the point of departure (PoD) to assess the carcinogenicity risk of PAs (EFSA, 2017), based on an updated benchmark dose (BMD) modelling approach. This was done because it was considered that the $\mathrm{BMDL}_{10}$ of $70 \mu \mathrm{g} / \mathrm{kg} /$ day obtained previously for lasiocarpine (EFSA, 2011) was affected by a high degree of uncertainty. In this study the MOE values were calculated by dividing the $\mathrm{BMDL}_{10}$ of $237 \mu \mathrm{g} / \mathrm{kg}$ bw/day for riddelliine by the EDIs.

\section{Real life exposure scenario}

MOE values for the chronic lifetime exposure to herbal teas and herbal medicines were calculated based on daily exposure during a lifetime. We applied Haber's rule to correct the EDI values for this short term exposure (Doull and Rozman, 2000). According to Haber's rule, the toxic effect varies linearly with the time of exposure and the concentration or dose (i.e. $\mathrm{C} \times \mathrm{T}=\mathrm{k}$, where $\mathrm{C}$ is concentration or dose, $\mathrm{T}$ is time of exposure, and $\mathrm{k}$ is a constant toxic response for the specific substance) (Doull and Rozman, 2000). Using Haber's rule and a lifetime expectancy of 75 years (Felter et al., 2011; van den Berg et al., 2014), the EDI values for two weeks yearly exposure during a lifetime will be 52 weeks per year $/ 2$ weeks $=26$ times lower than for daily lifelong exposure. EDI values for 6 weeks exposure a year, defined by the German and Netherlands regulations as short term exposure, would be 8.67 times lower. Another consideration to take into account when considering real life exposure scenario's for the use of herbal teas, is that these teas may not be ground before making the hot water extract. Using comminuted leaves is expected to facilitate diffusion of PAs into 
the hot water, and thus may result in a higher concentration of PAs in tea infusion then when using intact leaves. This will further influence the EDI and the MOE values. In the present study, five types of herbal teas were employed to compare the PA levels between comminuted leaves and intact leaves, as well as their resulting MOE values.

The possibility was considered that herbal teas or herbal medicines may be used for longer periods than 2 weeks or 6 weeks a year during a lifetime. For those herbal products, assuming a 75 -year lifetime, the maximum number of weeks was calculated during which the product could be consumed to result in an MOE value of 10,000 given a $\mathrm{BMDL}_{10}$ of $237 \mu \mathrm{g} / \mathrm{kg}$ bw per day:

$$
E D I=\frac{\text { BMDL10 }}{10,000}=\frac{\text { total } P \text { A concentration } * \text { daily use }}{b w(70 \mathrm{~kg})}:\left(\frac{75 \text { years } * 52 \text { weeks }}{n \text { weeks }}\right)
$$

This calculation was also applied for the 34 types of herbal teas and 19 PFS, based on the data that have been previously reported by Bodi et al. (2014), EFSA (2016) and Mulder et al. (2015). In all calculations it has been assumed that the concentrations reported are representative for the specific tea or PFS and that exposure to PAs is only due to that tea or PFS.

\section{Results}

\section{PA concentrations in herbal teas and the effect of hot water extraction of comminuted and intact leaves}

Five types of herbal teas were used to compare the total amount of PAs that were extracted either from the intact or the comminuted leaves. The amounts of PAs extracted from the intact leaves of each tea were consistently lower compared to the levels extracted from the comminuted leaves (Fig. 1). The total PA concentrations varied from 30.7 to $845.4 \mu \mathrm{g} / \mathrm{L}$ for the intact leaves and from 61.3 to 1120.0 $\mu \mathrm{g} / \mathrm{L}$ for the comminuted leaves. Overall, the PA levels extracted from intact leaves were 1.1- to 4.1fold lower than from the corresponding comminuted leaves. The PA levels were significantly different between intact leaves and comminuted leaves in borage, comfrey and climbing groundsel teas. The highest PA concentration was found in borage tea and lycopsamine N-oxide was the PA found in the highest concentration. In general, the same PAs and also similar profiles were found in intact and comminuted leaves of the teas (Table 3 and Supplementary Data 2). The tested herbal teas contained between 3 and 11 different PAs (Table 3 and Supplementary Data 2), the lowest number of PAs was detected in coltsfoot tea, the highest number in comfrey and sunn hemp tea. Interestingly, in sunn hemp tea trichodesmine N-oxide was the most abundant PA extracted from the intact leaves, whereas monocrotaline N-oxide was highest when extracted from comminuted leaves. This suggests that the size of the leaves may also have an impact on the relative extraction efficiency of PAs in the leaves.

We were interested whether the PAs present in the teas could be correlated with the botanical plant name listed on the label. Coltsfoot (T. farfara) is known to contain senkirkine as the dominant PA and varying but smaller amounts of senecionine (Roeder, 1995). In our sample senkirkine was indeed 
present but senecionine was not detected. Borago (B. officinalis) typically contains the monoesters lycopsamine and intermedine as well as their 7-acetyl derivatives (El-Shazly and Wink, 2014; Roeder, 1995). In the sample tested only lycopsamine and its 7-acetyl derivative were found. Comfrey (S. officinale) can contain a range of mono and diester compounds, including lycopsamine, intermedine, echinatine, echimidine, and heliosupine (El-Shazly and Wink, 2014). Lycopsamine, intermedine, echimidine and heliosupine were present in the tested sample, as well as leptanthine (a hydroxy analogue of lycopsamine) and an acetyl derivative of echimidine. Climbing groundsel (Senecio scandens) is reported to contain senecionine, seneciphylline (Roeder, 2000), although in another report adonifoline was identified as the main PA (Xiong et al., 2012). None of these PAs were detected in the tested sample. Based on the PA profile present in the extracts primarily senkirkine and lower levels of petasitenine and dehydrosenkirkine it is more likely to be a Petasitis (butterbur) species, e.g. $P$. japonicus (Hartmann and Witte, 1995). Sunn hemp (C. juncea) reportedly can contain trichodesmine, junceine, senecionine, integerrimine and seneciphylline (Roeder and Wiedenfeld, 2013). In the tested sample trichodesmine and integerrimine were indeed found, but senecionine, seneciphylline and junceine were not. Instead, monocrotaline and low levels of incanine and fulvine were detected. It is therefore possible that another or a mixture of Crotalaria species was used (Roeder and Wiedenfeld, 2013).

In several preparations trace levels PAs were found that could not be directly attributed to the botanical species reported on the label. These PAs can come from impurities present in the herbal teas, due to co-harvesting or processing of unrelated PA-containing plants.

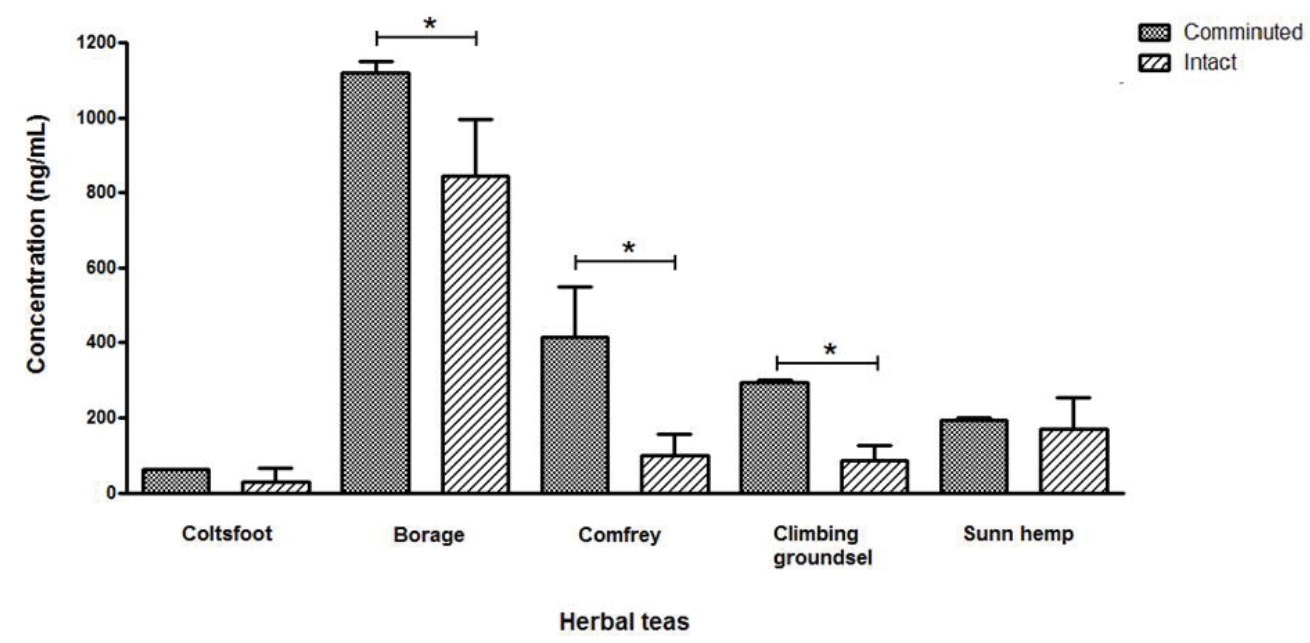

Fig.1 The total PA concentrations of five herbal teas extracted either from intact leaves (bars filled with black dots) or from the corresponding comminuted leaves (bars filled with black lines). Average of three extractions. Data are expressed as mean $\pm \mathrm{SD}$ and analyzed using $t$-test. ${ }^{*} \mathrm{p}<0.05$. 
Table 3. Total number of PAs, total PA concentration and the top three PAs in the tested herbal teas. See Supplementary Data 2 for a complete data overview.

\begin{tabular}{|c|c|c|c|c|c|}
\hline Herbal tea & Form & $\begin{array}{l}\text { Number of } \\
\text { PAs }>\text { LOD }\end{array}$ & $\begin{array}{l}\text { Total PA } \\
\text { concentration } \\
(\mu \mathrm{g} / \mathrm{L})\end{array}$ & $\begin{array}{l}\mathrm{EDI}(\mu \mathrm{g} / \mathrm{kg} \\
\text { bw/day) }\end{array}$ & $\begin{array}{l}\text { Top three PAs and their concentration } \\
(\mu \mathrm{g} / \mathrm{L})\end{array}$ \\
\hline \multirow{2}{*}{ Coltsfoot } & Comminuted & 5 & 61.3 & 0.1 & $\begin{array}{l}\text { Senkirkine (58.2); } \\
\text { Neosenkirkine }(2.6) ; \\
\text { Echinatine N-oxide }(0.27)\end{array}$ \\
\hline & Intact & 3 & 30.7 & 0.07 & $\begin{array}{l}\text { Senkirkine }(29.7) ; \\
\text { Neosenkirkine }(0.94) ; \\
\text { Echinatine N-oxide }(0.02)\end{array}$ \\
\hline \multirow{2}{*}{ Borage } & Comminuted & 4 & 1120.0 & 2.4 & $\begin{array}{l}\text { Lycopsamine N-oxide (889.3); } \\
\text { Lycopsamine (209.5); } \\
\text { 7-Acetyllycopsamine N-oxide (15.7) }\end{array}$ \\
\hline & Intact & 4 & 845.4 & 1.8 & $\begin{array}{l}\text { Lycopsamine N-oxide (679.7); } \\
\text { Lycopsamine (149.2); } \\
\text { 7-Acetyllycopsamine N-oxide (13.0) }\end{array}$ \\
\hline \multirow{2}{*}{ Comfrey } & Comminuted & 10 & 415.0 & 0.9 & $\begin{array}{l}\text { Echimidine N-oxide }(321.5) \\
\text { Echimidine }(29.1) \\
\text { Leptanthine }(22.7)\end{array}$ \\
\hline & Intact & 11 & 101.1 & 0.2 & $\begin{array}{l}\text { Echimidine N-oxide (75.9); } \\
\text { Echimidine (16.2); } \\
\text { Leptanthine N-oxide (4.0) }\end{array}$ \\
\hline \multirow{2}{*}{$\begin{array}{l}\text { Climbing } \\
\text { groundsel }\end{array}$} & Comminuted & 7 & 293.1 & 0.6 & $\begin{array}{l}\text { Senkirkine (248.2); } \\
\text { Dehydrosenkirkine (22.8); } \\
\text { Petasitenine (19.7) }\end{array}$ \\
\hline & Intact & 6 & 85.2 & 0.2 & $\begin{array}{l}\text { Senkirkine (74.3); } \\
\text { Petasitenine (5.0); } \\
\text { Dehydrosenkirkine (4.6) }\end{array}$ \\
\hline \multirow{2}{*}{ Sunn hemp } & Comminuted & 11 & 192.8 & 0.4 & $\begin{array}{l}\text { Monocrotaline N-oxide }(82.0) ; \\
\text { Monocrotaline }(60.1) ; \\
\text { Integerrimine N-oxide }(29.2)\end{array}$ \\
\hline & Intact & 9 & 170.0 & 0.4 & $\begin{array}{l}\text { Trichodesmine N-oxide (61.8); } \\
\text { Monocrotaline N-oxide (50.0); } \\
\text { Integerrimine N-oxide (18.7) }\end{array}$ \\
\hline
\end{tabular}

\section{Risk assessment for the herbal teas based on lifetime and shorter duration exposure}

The MOE values based on the total PA levels that were extracted from either comminuted leaves or intact leaves of the five types of herbal teas assuming daily use during a whole lifetime are depicted in Fig. 2A. It is assumed that the PA concentration is representative for the specific tea. For the comminuted leaves, the MOE values ranged from 100 to 1,800, and from 130 to 3,600 for the intact leaves. The MOE values were, regardless of the state and size of the leaves, all below 10,000 for these five types of herbal teas, the lowest MOE value was found for the borage tea.

Fig. 2B shows the MOE values for herbal teas in the form of comminuted leaves and intact leaves assuming consumption for two or six weeks/year during a lifetime. In particular consumption of borage tea still resulted in MOE values below 10,000, independent of the form of the leaves. Short term two weeks exposure to the extracts of comminuted leaves of comfrey and climbing groundsel teas resulted in MOE values just below 10,000, but for the intact leaves above 10,000. For coltsfoot and sunn hemp teas MOE values above 10,000 were obtained, irrespective of the form of the leaves. 
Assuming six weeks exposure/year during a lifetime, to mimic the definition of short term exposure in existing regulations, reduces the MOE values 3 -fold resulting in a value $<10,000$ for borage, comfrey and sunn hemp teas, independent of the form of the leaves. These results show that the size of the leaves and the duration of the short term exposure may influence the corresponding risk assessment.
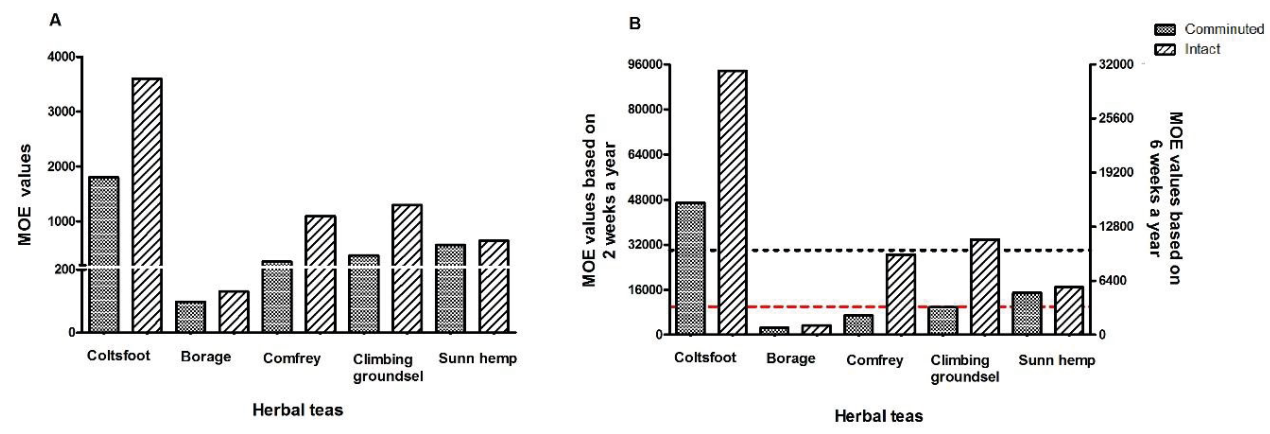

Fig.2 The MOE values of five types of herbal teas obtained when assuming daily consumption of one cup of tea per day for a lifetime (A) and for 2 weeks (left Y axis) and 6 weeks (right Y axis) every year during a lifetime (B) using the total PA levels extracted from either comminuted leaves (bars filled with black dots) or intact leaves (bars filled with black lines). The red dashed line (----) and black dotted line ( $\cdots \cdot)$ ) represent the MOE values of 10,000 for 2 weeks and 6 weeks a year, respectively.

\section{PA concentrations in herbal medicines}

Table 4 and Supplementary data 3 show the total PA concentrations found in eight herbal medicines, including four products (M5-8) that contain PA-producing plants such as coltsfoot or climbing groundsel as one of the ingredients. The other four herbal medicines (M1-4) were supposed to contain no PA-producing plants. In M1 and M2 the total PA levels were indeed below LOQ. A small amount of senkirkine $(4.2 \mu \mathrm{g} / \mathrm{kg})$ was found in $\mathrm{M} 3$ and traces of lycopsamine $/$ intemedine $(4.0 \mu \mathrm{g} / \mathrm{kg})$ were detected in M4. In the medicines that contain a PA-producing plant ingredient, the measured total PA concentration ranged from 404 to $7883 \mu \mathrm{g} / \mathrm{kg}$. Medicine 6 contained the highest total PA concentration as well as the highest number of different PAs. In this sample adonifoline was the dominant PA, accounting for approximately $98 \%$ of the total PA concentration. M5 and M8 contained coltsfoot (T. farfara) as one of the ingredients and in accordance with this, senkirkine, its isomer neosenkirkine, senecionine and its isomer integerrimine, were found as the main PAs (Roeder, 1995; Roeder, 2000). The results for M6 and M7, both containing climbing groundsel (S. scandens) as an ingredient, were more diverse. The total PA concentration in M7 was only 5\% of that of M6, what could be due to different inclusion levels of climbing groundsel in the products. However, also the PA profile in both medicines was quite different. M6 contained, besides the high concentration of adonifoline mentioned above, also lower levels of senecionine, seneciphylline and the monoesters lycopsamine, intermedine, echinatine and rinderine. The first three compounds have been reported for climbing groundsel (Roeder, 2000; Xiong et al., 2012). The monoesters are probably due to a 
contamination of the product (or an undeclared ingredient) with an Eupatorium or Boraginaceae species. The same monoesters were also found in M7, indicating a similar contamination, but for the rest this sample contained only senkirkine and lower levels of adonifoline.

Table 4. Total number of PAs, total PA concentration and the top three PAs in the tested herbal medicines. See Supplementary Data 3 for a complete data overview.

\begin{tabular}{|c|c|c|c|c|}
\hline $\begin{array}{l}\text { Herbal } \\
\text { medicine }\end{array}$ & $\begin{array}{l}\text { Number of PAs } \\
\text { detected }\end{array}$ & $\begin{array}{l}\text { Total PA concentration } \\
(\mu \mathrm{g} / \mathrm{kg})\end{array}$ & $\begin{array}{l}\mathrm{EDI}(\mu \mathrm{g} / \mathrm{kg} \\
\text { bw/day) }\end{array}$ & $\begin{array}{l}\text { Top three PAs and their } \\
\text { concentration }(\mu \mathrm{g} / \mathrm{kg})\end{array}$ \\
\hline M1 & 0 & $<\mathrm{LOQ}$ & - & - \\
\hline M2 & 0 & $<$ LOQ & - & - \\
\hline M3 & 1 & 4.2 & 0.0003 & Senkirkine (4.2) \\
\hline M4 & 1 & 4.0 & 0.001 & Lycopsamine/intermedine (4.0) \\
\hline M5 & 6 & 6344.3 & 2.18 & $\begin{array}{l}\text { Senkirkine (5369.3); Neosenkirkine } \\
\text { (868.9); Senecionine (79.7) }\end{array}$ \\
\hline M6 & 8 & 7883.2 & 0.59 & $\begin{array}{l}\text { Adonifoline (7734.3); } \\
\text { Seneciphylline (67.4); Senecionine } \\
(39.9)\end{array}$ \\
\hline M7 & 7 & 403.9 & 0.02 & $\begin{array}{l}\text { Senkirkine (292.1); } \\
\text { Adonifoline (49.7); } \\
\text { Echinatine (41.4) }\end{array}$ \\
\hline M8 & 4 & 1430.5 & 0.45 & $\begin{array}{l}\text { Senkirkine (1215.1); Neosenkirkine } \\
\text { (199.2); Senecionine (10.8) }\end{array}$ \\
\hline
\end{tabular}

-: Cannot be calculated because PA levels were $<$ LOQ.

\section{Risk assessment for the tested herbal medicines based on chronic exposure and short term exposure scenarios}

The MOE values for the eight types of medicines were evaluated according to three exposure scenario's including consumption at the recommended daily intake of that medicine daily throughout the whole lifespan (Fig. 3A), or shorter-than-lifetime during two or six weeks/year for 75 years, assuming a representative PA concentration and assuming exclusive exposure (Fig. 3B). Since M3 and M4 each only contained one PA at low concentrations, use of these two herbal medicines resulted in MOE values far above 10,000 irrespective of the duration of the exposure. However, of the four PAproducing plant containing herbal medicines, three samples resulted in low MOE values of between 110 and 530 when assuming lifelong daily consumption. For medicine 5 even short term consumption of two weeks/year resulted in an MOE value of 2800, well below 10,000, indicating that this medicine may pose a potential risk for human health. Considering shorter-than-lifetime exposure for 6 weeks/year resulted in MOE values $<10,000$ for three medicines containing PA plant material. 

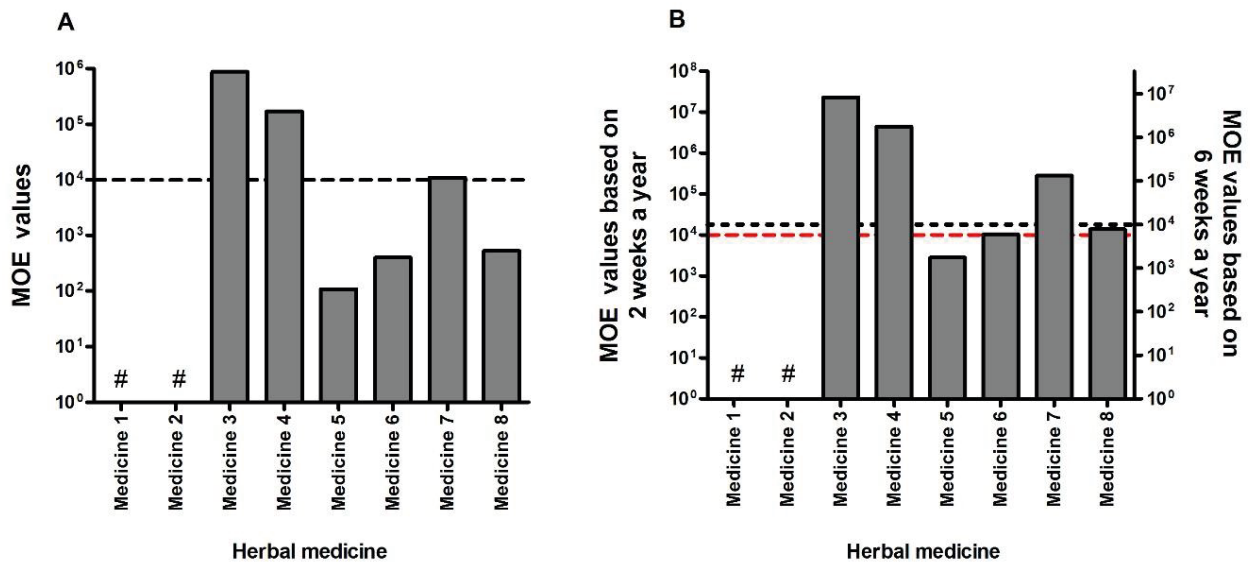

Fig. 3 The MOE values of eight different types of herbal medicines assuming lifelong daily consumption (A), and for 2 weeks (left Y axis) and 6 weeks (right Y axis) every year during a lifetime (B). The red dashed line (----) and black dotted line $(\cdots \cdots)$ represents the MOE values of 10,000 for 2 weeks and 6 weeks a year, respectively. \# implies PA content $<$ LOQ, no MOE values obtained.

\section{Risk assessment for the herbal teas, herbal medicine and previously analysed teas and PFS based on shorter-than-lifetime use}

In addition to the herbal teas and herbal medicines analysed in the present study also a risk assessment for shorter-than-lifetime exposure was made for the 34 types of (herbal) teas and 19 PFS for which PA levels were previously reported by Bodi et al. (2014), EFSA (2016) and Mulder et al. (2015). Given that the number of weeks a year selected for this shorter-than-life time exposure influences the MOE values and final conclusion, in this analysis the number of weeks during a lifetime that would result in an MOE of 10,000 was evaluated. The five types of herbal teas and eight types of herbal medicines analysed in the present study were also included in this evaluation.

Fig. 4 presents the maximum number of weeks during a 75-year lifetime that a herbal tea from the present study (Fig. 4A), a herbal medicine from the present study (Fig. 4B), a herbal tea analysed in previous studies (Fig. 4C) or a PFS sample analysed before (Fig. 4D) could be consumed to result in an MOE value of 10,000. From these data it follows for example that consumption of coltsfoot tea prepared from comminuted leaves, containing the PA concentration as determined in this study, for up to 700 weeks during a lifetime (corresponding to 9.3 weeks/year during 75 years) would be of little concern, whereas use of intact leaves to prepare the tea would increase this to 1400 weeks during a lifetime (18 weeks/year) (Fig. 4A). Similarly, use of intact borage, comfrey, climbing groundsel and sunn hemp tea leaves would raise no concern for, respectively, 50, 425, 500 and 250 weeks during a life time (corresponding to $0.6,5.6,6.7$ and 3.3 weeks/year, respectively). However, use of comminuted borage, comfrey, climbing groundsel and sunn hemp tea, would result in an acceptable 
exposure for, respectively, 40, 100, 150 and 225 weeks during a lifetime (corresponding to $0.5,1.3$, 2.0 and 3.0 weeks/year) (Fig. 4A).

Consumption of herbal medicines that contain a PA-producing plant as ingredient, would not raise a concern for medicines 5, 6 and 8 when consumption is less than approximately 40, 150 and 200 weeks during a lifetime $(0.5,2.0$ and 2.7 weeks/year). The other herbal medicines could be consumed on a daily basis, provided the medicine is the only source of PA exposure (Fig. 4B).

For the herbal teas for which PA levels were reported by Bodi et al. (2014), EFSA. (2016) and Mulder et al. (2015), 29 out of in total of 34 types of herbal teas displayed a maximum number of weeks exceeding a lifetime of 3900 weeks, assuming a daily consumption of one cup of tea (Fig 4C). Of the regular (herbal) tea types, only rooibos tea appeared to be contaminated with PA levels that would require them to be consumed shorter-than-life-time, for not more than 1750 weeks (23.3 weeks a year) to be of low concern. Regarding the 5 types of herbal teas, derived from PA-producing plants, the acceptable exposures ranged from 110 weeks (1.5 weeks a year) for borage tea to more than lifetime consumption for Eupatorium tea (Fig. 4C). The exposures calculated for borage tea and coltsfoot tea (900 weeks or 12 weeks/year) were in the same range as calculated for the samples of the same type in this study.

With respect to the PFS, 17 of 19 PFS showed that a daily use of $200 \mathrm{mg}$ during a lifetime would not raise a concern, and only for two samples, a plant extract formula reported by EFSA. (2016) and a plant extract formula (PA-plant) reported by Mulder et al. (2015), the consumption should be substantially shorter than lifetime, 690 weeks (9.2 weeks/year), and 160 weeks (2.1 week/year), respectively (Fig. 4D).

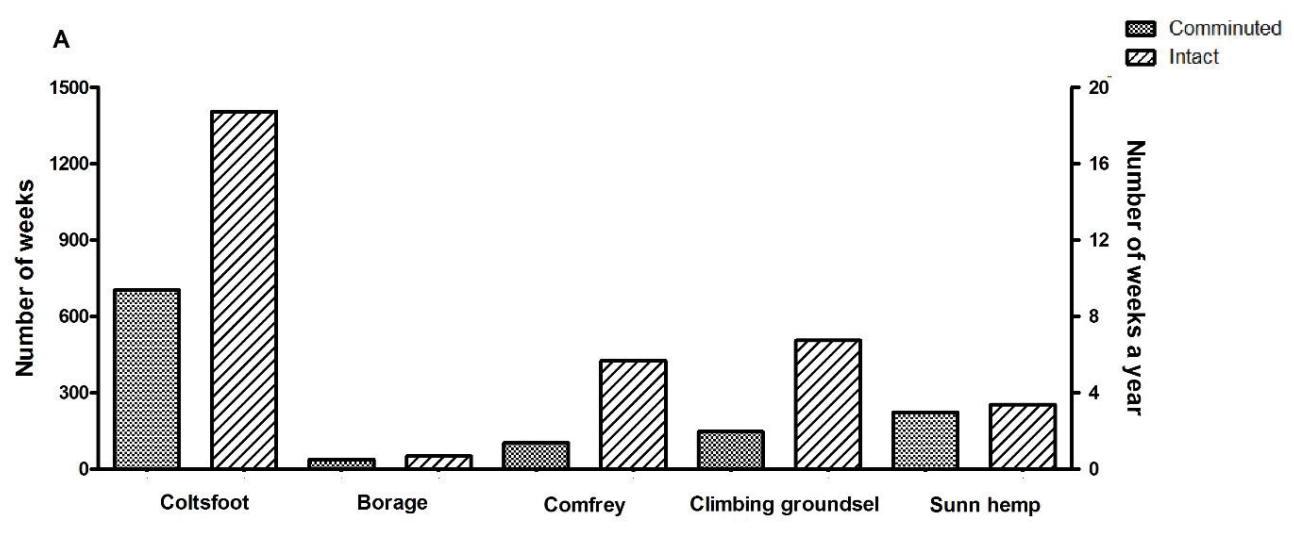

Herbal teas 


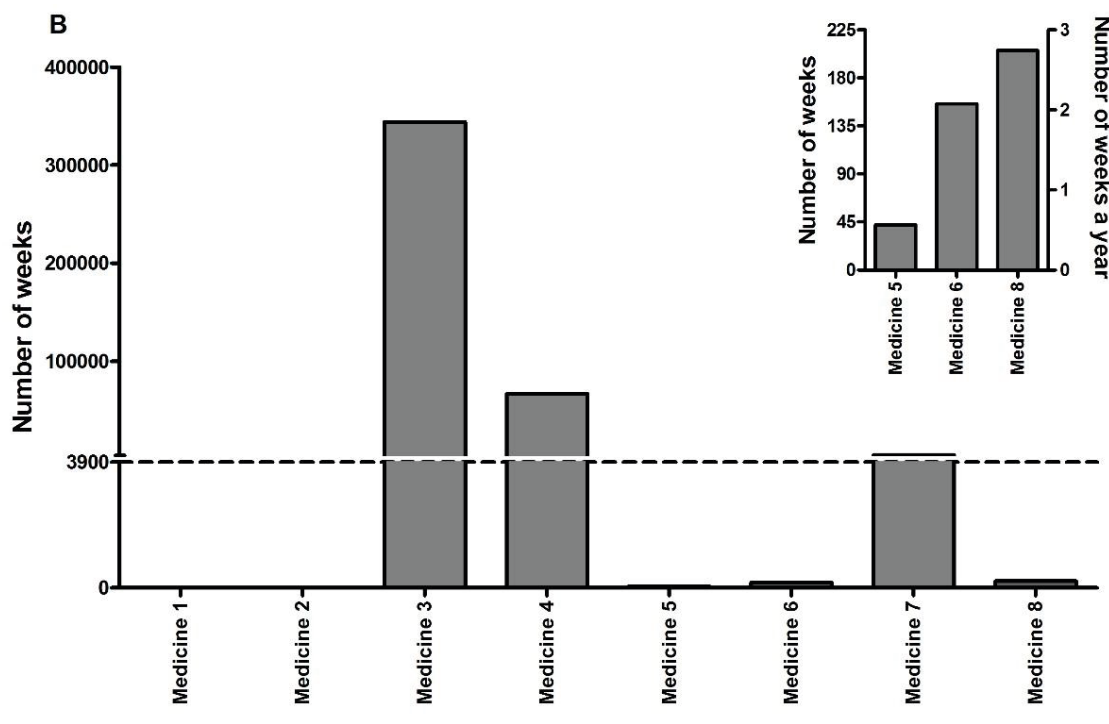

Herbal medicines

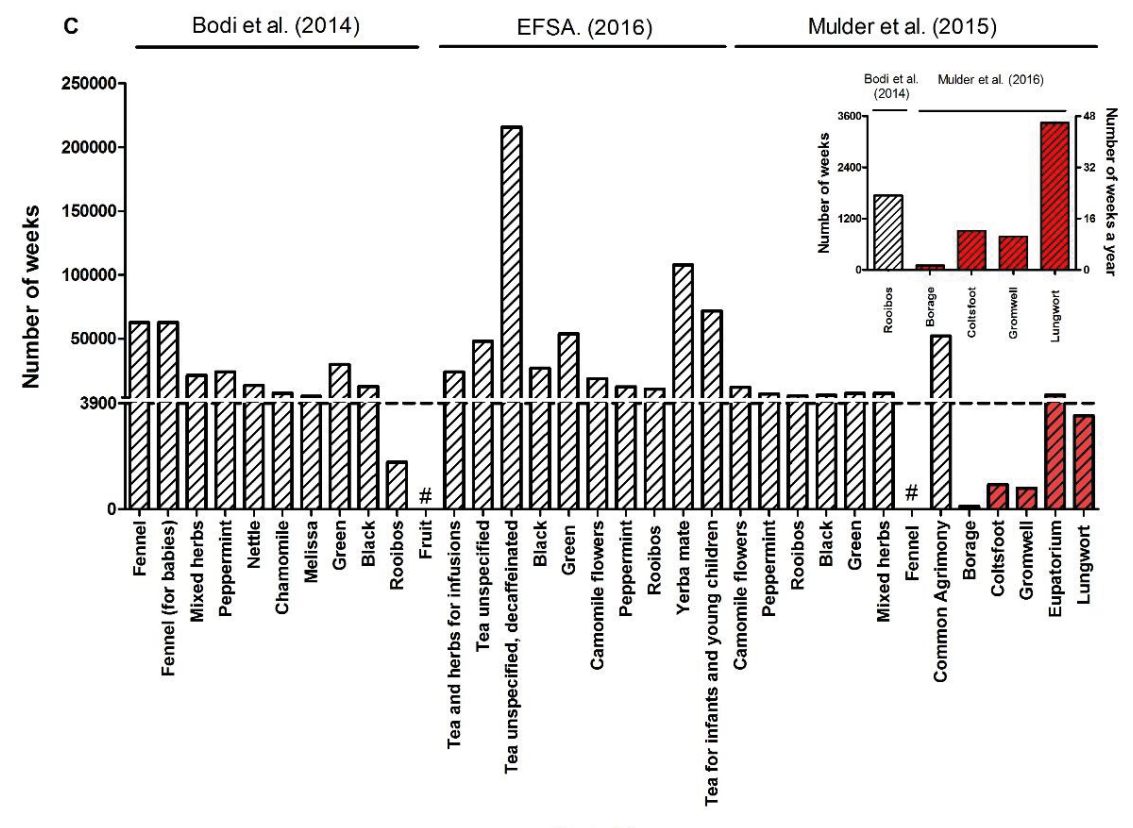

Herbal teas 


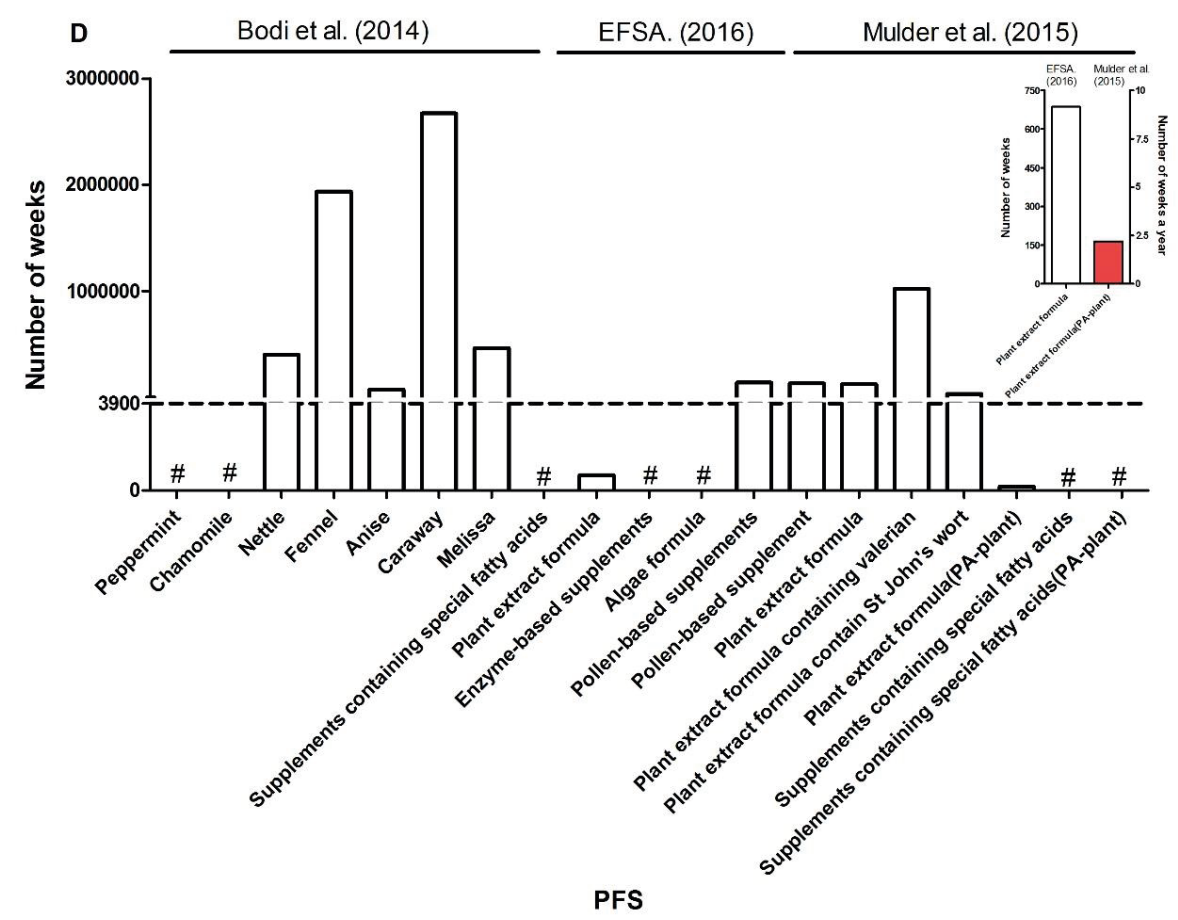

Fig. 4 The maximum number of weeks during a 75-year lifetime that a tea analysed in the present study (A), a medicine analysed in present study (B), a type of tea reported in the literature (C) or a type of PFS reported in the literature (D) could be consumed to result in an MOE of 10,000, assuming consumption of one cup of tea (150 $\mathrm{mL}$ ) or $200 \mathrm{mg}$ PFS. Bars filled with black dots represent the herbal teas prepared from comminuted leaves, bars filled with black slanted lines represent the herbal teas from intact leaves. The red bars represent the samples derived from PA-producing plants. The black dashed line represents daily intake during a 75 year lifetime (equal to 3900 weeks). \# indicates that data are not quantifiable due to a PA content $<$ LOQ.

\section{Discussion}

In the present study, we investigated the extraction of PAs in hot water from intact leaves and the corresponding finely ground leaves of five herbal PA containing teas and also examined the presence of PAs in eight samples of commercially available herbal medicines. For preparation of the infusion, the tea sample was steeped in the water for 10 minutes and the infusion was stirred 3 times. This procedure was selected as a worst case scenario for extraction of bioactive ingredients upon hot water extraction. For instance, Molan et al. (2009) investigated the effects of infusion time and stirring on the total phenolic levels extracted from green teas. The results showed that the total phenolic contents increased from $52.76 \pm 2.19$ to $102.83 \pm 2.33 \mathrm{mg} / \mathrm{g}(95 \%)$ by increasing the infusion time of 3 minutes to 10 minutes at $100{ }^{\circ} \mathrm{C}(\mathrm{P}<0.0001)$. The extraction was not further improved upon increasing the infusion time from 10 minutes to 30 minutes, at which time point the total phenolic levels was $106.8 \pm 3.29 \mathrm{mg} / \mathrm{g}$. In addition, infusion with stirring resulted in an increased total phenolic level of 
$96.12 \pm 2.67 \mathrm{mg} / \mathrm{g}$, which is increased by $37 \%$ compared to a total phenolic level of $70.39 \pm 0.27 \mathrm{mg} / \mathrm{g}$ resulted from infusion without stirring $(P \leqslant 0.0110-0.0001)$ (Molan et al., 2009). Similarly, McKAY et al. (1995) found that stirring during tea infusion resulted in a marked increase on oxalate concentrations in black teas (McKAY et al., 1995). EFSA also pointed out that the infusion time and stirring may have an influence on the extraction of PAs during consumer preparation (EFSA, 2016). Raffo et al. (2011) suggested that stirring 3 times could reflect best the scenario of preparing tea infusions by the consumer in general (Raffo et al., 2011).

In order to minimize the risk to miss relevant PAs, the samples were analysed for a comprehensive set of 89 1,2-unsaturated PAs. The previous studies on exposure and risk assessment for herbal teas and supplements were based on a set of 28 PAs (Bodi et al. (2014), EFSA (2016), Mulder et al. (2015)). The herbal teas and medicines investigated in this study, contained several PAs, that had not been included in previous studies, such as adonifoline, trichodesmine N-oxide, petasitenine, isomers of senkirkine and isomers of intermedine and lycopsamine. Herbal medicine M6 contained adonifoline in substantial amounts. When analysed with the set of 28 PAs, for this sample a relatively low amount of $131 \mu \mathrm{g} / \mathrm{kg}$ would have been found, instead of $7883 \mu \mathrm{g} / \mathrm{kg}$ with this method (Supplementary Data 3). In the other PA-containing medicines, the set of 28 PAs accounted for $74 \%$ to $86 \%$ of the total content found using the comprehensive set. With respect to the five herbal teas, the difference in total PA concentration was relatively small, except for sunn hemp tea. For this tea the 28 PAs accounted for $42 \%$ (intact leaves) to $75 \%$ (comminuted leaves), while for the other preparations it ranged from $85 \%$ to $98 \%$ (Supplementary Data 2). It may be concluded that the set of 28 PAs is not always sufficient to get a reliable impression of the PAs present in these herbal teas and medicines.

The herbal teas selected in this study are available for the consumer in the form of intact leaves. Comparing total PA levels in hot water extracts of the intact and comminuted leaves from five PAplant containing herbal teas, we found that, overall, the PA concentrations extracted from the comminuted leaves were 1.1 to 4.1 times higher compared to concentrations extracted from the intact leaves. It is of interest that, not only higher levels of PAs were extracted from the comminuted leaves but sometimes also different PAs were found in the hot water extracts from the comminuted leaves. This indicates that PA extraction efficiency can be influenced by the particle size of the botanical sample. In line with this, previous studies have demonstrated that using comminuted fruits increased the extraction efficiency of alkenylbenzenes as compared to the whole fruits (Raffo et al., 2011; van den Berg et al., 2014). The results of the current study suggest that the use of comminuted tea materials to prepare hot water extracts, as routinely done in studies on PAs in herbal teas (Bodi et al., 2014; EFSA, 2016; Mulder et al., 2015) may overestimate the levels and thus, also the corresponding exposure and risk. It was noted that hot water extraction from intact leaves resulted in a larger variation in the PA levels than observed for comminuted leaves. This may be due to the fact that PA levels can vary between the individual intact leaves, which are likely to originate from different plants. 
The leaves may also originate from different batches of teas, from different locations, which were mixed during processing and packaging. Therefore, it is conceivable that the PA levels of intact leaves derived from a single bag of tea are variable. Grinding the leaves and homogenizing the ground product will strongly reduce differences in the samples.

It is of interest to note that the form of the leaves also may have an effect on the type of PAs extracted. For example trichodesmine N-oxide was the PA present at the highest concentrations in sunn hemp teas extracted from the intact leaves, whereas monocrotaline N-oxide was the most abundant PA in the extract from the comminuted leaves. In hot water extracts from intact and comminuted leaves the amount of PA N-oxides dominated over that of PA free bases, which is in line with other studies However, in the herbal medicines the PAs were present solely in the free-base form. In line with this, Griffin et al. (2014) reported that only 6 samples out of a total of 54 herbal medicines contained PA Noxides (Griffin et al., 2014).

Using the total PA levels, we performed a risk assessment for these herbal products taking the use of intact tea leaves as well as shorter-than-lifetime exposure scenario's into account. In addition, we analysed the consequences of shorter-than-lifetime use for the risk assessment of herbal teas and PFS reported by Bodi et al. (2014), EFSA (2016), and Mulder et al. (2015).

We previously found that whole lifetime exposure to PA-plant containing herbal teas, including borage and coltsfoot teas with consumption of one cup of those teas per day would result in the MOE values below 10,000 (Chen et al., 2017). However, results of the present study indicate that when considering shorter-than-lifetime exposure the outcome of the risk assessment may be different. When use of the herbal teas analysed in the present study was assumed to be limited to 2 weeks a year during a lifetime, coltsfoot tea was shown to result in an MOE value higher than 10,000. Considering use during 2 (or 6) weeks a year for a lifetime increased the MOE values 26- (or 8.67-) fold, while using the $\mathrm{BMDL}_{10}$ for riddelliine of $237 \mu \mathrm{g} / \mathrm{kg}$ bw per day instead of that for lasiocarpine of $70 \mu \mathrm{g} / \mathrm{kg}$ bw per day in our previous study, additionally increased the MOE values by a factor of 3.4 (EFSA, 2017).

We also estimated the number of weeks one could consume the different types of teas and PFS that have been analysed before by Bodi et al. (2014), EFSA (2016) and Mulder et al. (2015) during a 75year lifetime. The results showed that the regular consumption of herbal teas derived from PAproducing plants except for eupatorium tea would not raise a concern when consumed for periods varying from 40 to 3450 weeks during a lifetime, which is equivalent to 0.5 to 46 weeks/year for 75 years. Obviously, the outcome strongly depends on the average PA content in these teas and the amount of tea consumed. Similarly, the weeks of regular consumption of PFS that would not raise a concern varied from 160 to 690 weeks during a life time, equivalent to 2.1 to 9.2 weeks a year during 75 years.

It is of interest to note that the values from 2.1 to 9.2 weeks a year obtained for safe consumption of PA containing PFS, are all covered by the limit of two weeks established by EMA for the short term 
use of such herbal products in self-medication (EMA, 2007), but not fully covered by the 6 weeks defined as short term exposure in the German and Netherlands regulation (Bundesgesundheitsamt, 1992; WKB, 2001). This regulation however also defines a maximum daily use of $1 \mu \mathrm{g} /$ day. This means that a yearly 6-weeks intake of $1 \mu \mathrm{g}$ /day by a $70 \mathrm{~kg}$ person would result in an MOE of about 144,000 , and thus not raise a concern. In fact, an intake of $1 \mu \mathrm{g} /$ day during a lifetime by a $70 \mathrm{~kg}$ person would result in an MOE of 16,600, which is still a sufficient safety margin. It is of interest to note that the PA content in the herbal products evaluated in the present study can result in intakes far beyond the levels specified in regulations for PAs in botanicals and botanical preparations. As outlined above this includes the regulations in Germany and the Netherlands, where the government has established maximum limits for daily intake of $1 \mu \mathrm{g}$ /day (for short term use up to 6 weeks) and 0.1 $\mu \mathrm{g} / \mathrm{day}$ (for long term use) and a maximum PA content for herbal supplements of $1 \mu \mathrm{g} / \mathrm{kg}$ or $1 \mu \mathrm{g} / \mathrm{L}$ (Bundesgesundheitsamt, 1992; WKB, 2001). In Austria and Belgium, this regulation is even more strict, with a "zero tolerance" approach towards PAs (Bundesgesetzblatt, 1994; Koninklijk besluit, 1997). So far there are no maximum limits established in the EU for daily PA intake (other than what is specified for specific medicinal herbal products). The EU is currently considering maximum limits (ML) for PAs in teas and supplements in general. These maximum limits will likely be based on the evaluation made by EFSA regarding the occurrence of PAs in herbal teas and PFS, and the consumption of these products by different age and population groups (EFSA, 2016; EFSA, 2017). Depending on the level of safety that is pursued and the evaluation on what is reasonably achievable in lowering the contamination levels in these products, it is not unlikely that the maximum limits will be set in the range of 100 to $500 \mu \mathrm{g} / \mathrm{kg}$, with the possibility that different MLs may be set for different (types of) products. On the other hand, in other countries such as China, there are no specific regulations for the maximum limits of daily PA intake (CP, 2010). This situation may raise a possible concern for human health especially when these preparations would be used for longer periods of time. In the current study we focused primarily on the individual MOEs for a set of herbal teas and medicines, assuming that there is no additional exposure to PAs from any other source. One could argue that our approach for herbal teas and PFS could be prone to underestimate the risks in terms of the amounts of PAs consumed, since individuals may drink different types of herbal teas at the same time with addition of honey, a product which contains PAs (EFSA, 2011). Importantly, according to the EFSA risk assessment report, there are consumer groups that have a relatively high exposure to PAs due to consumption of contaminated teas and honey (EFSA, 2017). It is conceivable that these consumers may be at greater risk in case that they would also use the PA-containing herbal products such as PFS. A more complex scenario could be developed that considers short exposure to combinations of herbal teas, PFS, or other herbal products which are derived from PA-producing plants. More detailed information on consumption habits of herbal teas by the average population and by the $95^{\text {th }}$ percentile population (heavy consumers) should be collected to further evaluate the 
influence of shorter-than-lifetime exposure scenario's. However, at the moment data regarding combined consumption of different types of herbal preparations are mostly lacking, which makes the related risk assessment difficult to perform. Such data await to be collected and will contribute to risk assessment for the current exposure to PAs.

Taken together the results of the present study illustrate the need for development of a generally accepted method for taking shorter-than-lifetime exposure into account when analysing the risks of botanicals and botanical preparations containing compounds that can be genotoxic and carcinogenic. Using Haber's rule to correct for shorter periods of use may prevent from an overestimation of the actual risk to human health. The application of Haber's rule is based on the assumption that the tumour incidence and carcinogenic processes induced by carcinogens have a linear relationship with the cumulative dose (Crump et al., 1976). At present there is only limited data available that supports such a linear dose-response relationship for tumour formation. Currently, there is no valid method on how to take a shorter-than-lifetime exposure into account in an MOE based risk assessment. Nevertheless using Haber's rule gives a reasonable first approach to assess the risks related to shorter-than-lifetime exposure. It is obvious that future developments in risk assessment should consider how to further advance this issue of taking shorter-than-lifetime exposure into account when applying the MOE approach in risk assessment of compounds that are both genotoxic and carcinogenic.

\section{Acknowledgements}

This work was funded by a grant from the China Scholarship Council to Lu Chen (grant No. 201508650023). 


\section{References}

Ancuceanu, R., Dinu, M., Arama, C., 2013. Weight loss food supplements: adulteration and multiple quality issues in two products of Chinese origin. Farmacia 61, 28-44.

Ashok, P., Sinnathambi, A., Hulkoti, B., Desai, B., 2006. Anti-inflammatory and Anti-ulcerogenic effect of Crotalaria juncea Linn. in albino rats. Iranian journal of pharmacology and therapeutics 5, 141-140.

BfR, 2013. Pyrrolizidine alkaloids in herbal teas and teas. BfR Opinion No. 018/2013 of 5 July 2013. Pyrrolizidine alkaloids in herbal teas and teas.

Bodi, D., Ronczka, S., Gottschalk, C., Behr, N., Skibba, A., Wagner, M., Lahrssen-Wiederholt, M., Preiss-Weigert, A., These, A., 2014. Determination of pyrrolizidine alkaloids in tea, herbal drugs and honey. Food Additives \& Contaminants: Part A 31, 1886-1895.

Bundesgesundheitsamt, 1992. Bekanntmachung über die Zulassung und Registrierung von Arzneimitteln. Bundesanzeiger 111, 4805.

Bundesgesetzblatt, 1994. Verordnung des Bundesministers für Gesundheit und öffentlicher Dienst vom 5. Mai 1989 betreffend Arzneimittel, die nicht in Verkehr gebracht werden dürfen, 555/1994, Vienna, Austria.

Carvalho, L.D., Cohen, P., Silva, C., Moreira, A., Falcão, T., Dal Molin, T., Zemolin, G., Martini, M., 2012. A new approach to determining pharmacologic adulteration of herbal weight loss products. Food Additives \& Contaminants: Part A 29, 1661-1667.

Chen, L., Mulder, P.P.J, Louisse, J., Peijnenburg, A., Wesseling, S., Rietjens, I.M., 2017. Risk assessment for pyrrolizidine alkaloids detected in (herbal) teas and plant food supplements. Regulatory Toxicology and Pharmacology 86, 292-302.

Chou, M.W., Wang, Y.-P., Yan, J., Yang, Y.-C., Beger, R.D., Williams, L.D., Doerge, D.R., Fu, P.P., 2003. Riddelliine N-oxide is a phytochemical and mammalian metabolite with genotoxic activity that is comparable to the parent pyrrolizidine alkaloid riddelliine. Toxicology letters $145,239-247$.

Crump, K., Hoel, D., Langley, C., Peto, R., 1976. Fundamental carcinogenic processes and their implications for low dose risk assessment. Cancer research 36, 2973-2979.

CP Commission. 2010. Pharmacopoeia of the People's Republic of China. China Medical Science Press, Beijing.

Doull, J., Rozman, K., 2000. Using Haber's Law to define the margin of exposure. Toxicology 149, 1.

Edgar, J., Lin, H., Kumana, C., Ng, M., 1992. Pyrrolizidine alkaloid composition of three Chinese medicinal herbs, Eupatorium cannabinum, E. japonicum and Crotalaria assamica. The American journal of Chinese medicine 20, 281-288.

EFSA, 2005. Opinion of the Scientific Committee on a request from EFSA related to a harmonised approach for risk assessment of substances which are both genotoxic and carcinogenic. EFSA Journal 3, 282.

EFSA, 2011. Scientific opinion on pyrrolizidine alkaloids in food and feed. EFSA Journal 9, 2406.

EFSA, 2012. Guidance on selected default values to be used by the EFSA Scientific Committee, Scientific Panels and Units in the absence of actual measured data. EFSA journal 10, 2579.

EFSA, 2016. Dietary exposure assessment to pyrrolizidine alkaloids in the European population. EFSA Journal 14, e04572.

EFSA, 2017. Risks for human health related to the presence of pyrrolizidine alkaloids in honey, tea, herbal infusions and food supplements. EFSA Journal 15.

El-Shazly, A., Wink, M., 2014. Diversity of pyrrolizidine alkaloids in the Boraginaceae structures, distribution, and biological properties. Diversity 6, 188-282.

EMA, 2007. Committee on herbal medicinal products (HMPC) community herbal monograph on Foeniculum vulgare miller subsp. vulgare var. vulgare, fructus. (Doc. Ref: EMEA/HMPC/137428/2006 Corr.).

EMA, 2008. Committee on herbal medicinal products (HMPC) assessment report on Foeniculum vulgare Miller (Doc.Ref: EMEA/HMPC/137426/2006). 
Felter, S.P., Conolly, R.B., Bercu, J.P., Bolger, P.M., Boobis, A.R., Bos, P.M., Carthew, P., Doerrer, N.G., Goodman, J.I., Harrouk, W.A., 2011. A proposed framework for assessing risk from lessthan-lifetime exposures to carcinogens. Critical reviews in toxicology 41, 507-544.

Fu, P.P., Yang, Y.-C., Xia, Q., Chou, M.W., Cui, Y.Y., Lin, G., 2002. Pyrrolizidine alkaloidstumorigenic components in Chinese herbal medicines and dietary supplements. Journal of Food and Drug Analysis 10, 198-211.

Fu, P.P., Xia, Q., Lin, G. and Chou, M.W., 2004. Pyrrolizidine alkaloids-genotoxicity, metabolism enzymes, metabolic activation, and mechanisms. Drug metabolism reviews, 36(1), pp.1-55.

Garcia-Alvarez, A., Egan, B., de Klein, S., Dima, L., Maggi, F.M., Isoniemi, M., Ribas-Barba, L., Raats, M.M., Meissner, E.M., Badea, M., 2014. Usage of plant food supplements across six European countries: findings from the PlantLIBRA consumer survey. PloS one 9, e92265.

Griffin, C.T., Gosetto, F., Danaher, M., Sabatini, S., Furey, A., 2014. Investigation of targeted pyrrolizidine alkaloids in traditional Chinese medicines and selected herbal teas sourced in Ireland using LC-ESI-MS/MS. Food Additives \& Contaminants: Part A 31, 940-961.

Hartmann, T., Witte, L., 1995. Chemistry, biology and chemoecology of the pyrrolizidine alkaloids, Alkaloids: chemical and biological perspectives. Elsevier, pp. 155-233.

Hirono, I., Haga, M., Fujii, M., Matsuura, S., Matsubara, N., Nakayama, M., Furuya, T., Hikichi, M., Takanashi, H., Uchida, E., 1979. Induction of hepatic tumors in rats by senkirkine and symphytine. Journal of the National Cancer Institute 63, 469-472.

Hirono, I., Mori, H., Culvenor, C.C., 1976. Carcinogenic activity of coltsfoot, Tussilago farfara L. GANN Japanese Journal of Cancer Research 67, 125-129.

Kuhara, K., Takanashi, H., Hirono, I., Furuya, T., Asada, Y., 1980. Carcinogenic activity of clivorine, a pyrrolizidine alkaloid isolated from Ligularia dentata. Cancer letters 10, 117-122.

Koninklijk besluit, 1997. Koninklijk besluit van 29 Augustus 1997 betreffende de fabricage van en de handel in voedingsmiddelen die uit planten of uit plantenbereidingen samengesteld zijn of deze bevatten (Stbl. 21. XI. 1997).

Lapenna, S., Gemen, R., Wollgast, J., Worth, A., Maragkoudakis, P., Caldeira, S., 2015. Assessing herbal products with health claims. Critical reviews in food science and nutrition 55, 1918 1928.

Lin, G., Wang, J.Y., Li, N., Li, M., Gao, H., Ji, Y., Zhang, F., Wang, H., Zhou, Y., Ye, Y., 2011. Hepatic sinusoidal obstruction syndrome associated with consumption of Gynura segetum. Journal of hepatology 54, 666-673.

Ling, L., 2008. Study on the Identification of TLC for Chinese Herbal Medicine Groundsel. Chinese Journal of Ethnomedicine and Ethnopharmacy 9, 017.

Margalith, D., Heraief, C., Schindler, A., Birchler, R., Mosimann, F., Aladjem, D., Gonvers, J., 1985. Veno-occlusive disease of the liver due to the se of tea made from Senecio plants. J. Hepatol 204.

Mohabbat, O., Younos, M.S., Merzad, A., Srivastava, R., Sediq, G.G., Aram, G., 1976. An outbreak of hepatic veno-occlusive disease in north-western Afghanistan. The Lancet 308, 269-271.

Mulder, P.P.J, López, P., Castelari, M., Bodi, D., Ronczka, S., Preiss-Weigert, A., These, A., 2018. Occurrence of pyrrolizidine alkaloids in animal-and plant-derived food: results of a survey across Europe. Food Additives \& Contaminants: Part A 35, 118-133.

Mulder, P.P.J, Sánchez, P.L., These, A., Preiss - Weigert, A., Castellari, M., 2015. Occurrence of Pyrrolizidine Alkaloids in food. EFSA Supporting Publications 12, 859E.

Molan, A.L., De, S. and Meagher, L., 2009. Antioxidant activity and polyphenol content of green tea flavan-3-ols and oligomeric proanthocyanidins. International journal of food sciences and nutrition, 60(6), pp.497-506.

McKAY, D.W., Seviour, J.P., Comerford, A., Vasdev, S. and Massey, L.K., 1995. Herbal tea: an alternative to regular tea for those who form calcium oxalate stones. Journal of the Academy of Nutrition and Dietetics, 95(3), p.360.

NTP, 1978. Bioassay of lasiocarpine for possible carcinogenicity. National Cancer Institute carcinogenesis technical report series 39,1 . 
NTP, 2003. Toxicology and carcinogenesis studies of riddelliine (CAS No. 23246-96-0) in F344/N rats and B6C3F1 mice (gavage studies). National Toxicology Program technical report series, 1.

Oomah, B.D., Mazza, G., 1999. Health benefits of phytochemicals from selected Canadian crops. Trends in food science \& technology 10, 193-198.

Prakash, A.S., Pereira, T.N., Reilly, P.E., Seawright, A.A., 1999. Pyrrolizidine alkaloids in human diet. Mutation Research/Genetic Toxicology and Environmental Mutagenesis 443, 53-67.

Raffo, A., Nicoli, S., Leclercq, C., 2011. Quantification of estragole in fennel herbal teas: implications on the assessment of dietary exposure to estragole. Food and Chemical Toxicology 49, 370-375.

Reeuwijk, N.M., Venhuis, B.J., de Kaste, D., Hoogenboom, R.L., Rietjens, I.M., Martena, M.J., 2014. Active pharmaceutical ingredients detected in herbal food supplements for weight loss sampled on the Dutch market. Food Additives \& Contaminants: Part A 31, 1783-1793.

Rietjens, I.M., Slob, W., Galli, C., Silano, V., 2008. Risk assessment of botanicals and botanical preparations intended for use in food and food supplements: emerging issues. Toxicology letters 180, 131-136.

Roeder, E., 1995. Medicinal plants in Europe containing pyrrolizidine alkaloids. Pharmazie 50, 83-98.

Roeder, E., 2000. Medicinal plants in China containing pyrrolizidine alkaloids. Pharmazie 55, 711-726.

Roeder, E., Wiedenfeld, H., 2013. Plants containing pyrrolizidine alkaloids used in the Traditional Indian Medicine-including Ayurveda. Die Pharmazie-An International Journal of Pharmaceutical Sciences 68, 83-92.

Ruan, J., Gao, H., Li, N., Xue, J., Chen, J., Ke, C., Ye, Y., Fu, P.P.-C., Zheng, J., Wang, J., 2015. Blood pyrrole-protein adducts - a biomarker of pyrrolizidine alkaloid-induced liver injury in humans. Journal of Environmental Science and Health, Part C 33, 404-421.

Schoental, R., 1970. Hepatotoxic activity of retrorsine, senkirkine and hydroxysenkirkine in newborn rats and the role of epoxides in carcinogenesis by pyrrolizidine alkaloids and aflatoxins. Nature 227, 401-402.

Shumaker, R., Robertson, K., Hsu, I., Allen, J., 1976. Neoplastic transformation in tissues of rats exposed to monocrotaline or dehydroretronecine. Journal of the National Cancer Institute 56, 787-790.

Stegelmeier, B., Edgar, J., Colegate, S., Gardner, D., Schoch, T., Coulombe, R., Molyneux, R., 1999. Pyrrolizidine alkaloid plants, metabolism and toxicity. Journal of natural toxins 8, 95-116.

Stickel, F., Seitz, H.K., 2000. The efficacy and safety of comfrey. Public Health Nutrition 3, 501-508.

Tandon, B.N., Tandon, H., Tandon, R., Narndranathan, M., Joshi, Y., 1976. An epidemic of venoocclusive disease of liver in central India. The Lancet 308, 271-272.

van den Berg, S.J., Alhusainy, W., Restani, P., Rietjens, I.M., 2014. Chemical analysis of estragole in fennel based teas and associated safety assessment using the Margin of Exposure (MOE) approach. Food and chemical toxicology 65, 147-154.

Weston, C., Cooper, B., Davies, J., Levine, D., 1987. Veno-occlusive disease of the liver secondary to ingestion of comfrey. British medical journal (Clinical research ed.) 295, 183.

WKB, 2001. Warenwetbesluit Kruidenpreparaten Besluit van 19 Januari 2001, houdende vaststelling van het Warenwetbesluit Kruidenpreparaten. Staatsblad van het Koninkrijk der Nederlanden $2001,56,1-12$.

Xiong, A., Yang, L., Ji, L., Wang, Z., Yang, X., Chen, Y., Wang, X., Wang, C., Wang, Z., 2012. UPLC-MS based metabolomics study on Senecio scandens and S. vulgaris: an approach for the differentiation of two Senecio herbs with similar morphology but different toxicity. Metabolomics 8, 614-623. 


\begin{tabular}{|c|c|c|c|c|c|c|c|c|c|c|c|c|c|c|c|c|c|c|c|c|c|c|c|c|}
\hline 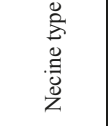 & 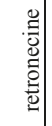 & 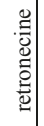 & & \begin{tabular}{l|l}
0 \\
0 \\
0
\end{tabular} & : & 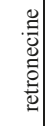 & 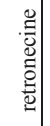 & & 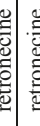 & 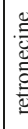 & $\frac{1}{0}$ & & & & & & & 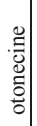 & 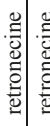 & 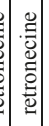 & | & 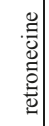 & |: & \\
\hline 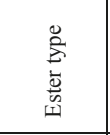 & $\begin{array}{l}0 \\
0 \\
0 \\
0 \\
0 \\
0 \\
\vdots \\
\end{array}$ & 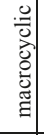 & \begin{tabular}{l|l}
0 \\
0 \\
0
\end{tabular} & 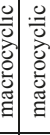 & 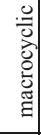 & 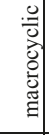 & 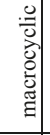 & & 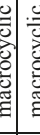 & & $\mid \mathbb{\Xi}$ & & & & & & & 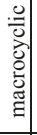 & 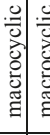 & 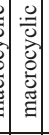 & 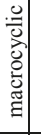 & 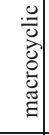 & 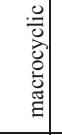 & \\
\hline 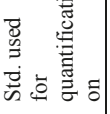 & 总 & $\begin{array}{l}0 \\
\text { के } \\
\text { a }\end{array}$ & & 至 $\widetilde{\approx}$ & 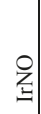 & 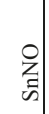 & 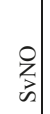 & & aी & \pm & $\Xi$ & & 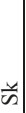 & $\approx$ & & & $\ddot{\omega}$ & $\frac{4}{\omega}$ & & & $\begin{array}{l}q \\
\vec{z} \\
z\end{array}$ & 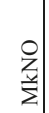 & 只 & $\omega$ \\
\hline 妾言 & -1 & - & -1 & $-1-$ & -1 & -1 & - & & - & & - & & & & & & -1 & - & - & - & - & - & - & - \\
\hline 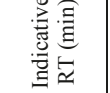 & ?ำ & $\begin{array}{l}\infty \\
\stackrel{\infty}{0} \\
i\end{array}$ & : & 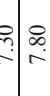 & ถู่ & ? & ?.n. & 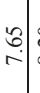 & $\begin{array}{c}0 \\
0 \\
\infty\end{array}$ & is & $\begin{array}{l}n \\
\infty \\
\infty\end{array}$ & & 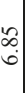 & & & & 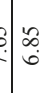 & $\stackrel{8}{\circ}$ & : & 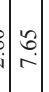 & \&. & $\stackrel{0}{\circ}$ & ఫ్ & f. \\
\hline ن & ले & m & ১) & $m$ & ¿ & 인 & ल & & 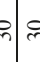 & ल) & in & & in & $\hat{\imath}$ & 8 & & $\approx$ & 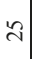 & $\dot{m} \equiv$ & $\ddot{m}$ & 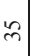 & $\approx$ & in & i \\
\hline 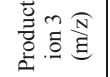 & $\stackrel{\infty}{=}$ & $\stackrel{\infty}{=}$ & $\begin{array}{c}\infty \\
\end{array}$ & $\stackrel{\infty}{\infty} \stackrel{\infty}{\sim}$ & 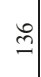 & $\cong$ & 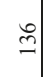 & むু) & $\stackrel{\infty}{2} \underset{\sim}{\infty}$ & $\underset{త ్}{\infty}$ & $\bar{\Xi}$ & & in & 8 & & & 0 & . & $\stackrel{\infty}{=} \underset{m}{\infty}$ & $\stackrel{\infty}{\infty}$ & $\stackrel{\infty}{=}$ & $\stackrel{\infty}{=}$ & $\stackrel{\overbrace{}}{9}$ & $\cong$ \\
\hline $\overrightarrow{0}$ & ল) & ले & ल) & $\stackrel{m}{m}$ & 이 & 이 & ల) & & $n$ & ल & & $\approx$ & ત્ & a & & & ఏ & 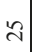 & el & i & 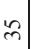 & $m$ & s & లి \\
\hline 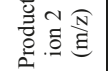 & ఫิ & $\stackrel{\infty}{=}$ & ปิ) & ิำ & ปิ & ฐิ & ฐิ & $\stackrel{n}{n}$ & తิ) & ฐ & สี & $\stackrel{\infty}{0}$ & $\stackrel{\infty}{\infty}$ & $\infty$ & & & 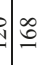 & 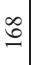 & తి తి & 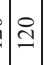 & ָิ & 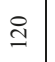 & తิ & $\stackrel{0}{0}$ \\
\hline రి & of & q & of & of & $q$ & of & q & ㅇ & of & 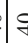 & $\approx$ & 요 & i & ¿ & q & & fi & ह & of & $q$ & q & q & q & ల్ల \\
\hline 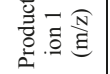 & ta & \$ & t & ¿ & J & \pm & स & & む) & ১ & ㄱ. & & ป & $\Xi$ & & & 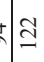 & $\Xi$ & む) & 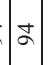 & ¿ & d & d & $\mathcal{I}$ \\
\hline 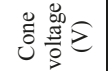 & q & f & q & of $q$ & q & q & $q$ & f & 8 f & f & f & & 요 & $m$ & & & m & in & q 9 & 8 & q & f & q & $\stackrel{m}{m}$ \\
\hline 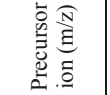 & ڤ్ & 畓| & \begin{tabular}{c|c}
$n$ \\
\hdashline \\
$m$
\end{tabular} & $\begin{array}{c}0 \\
0\end{array}$ & लें & ते & $\begin{array}{c}\text { तె } \\
\text { ले }\end{array}$ & $\stackrel{\overbrace{}}{n}$ & 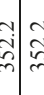 & in & 点 & (3) & |l| & 0 & & & 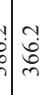 & శֶ. & & & $\begin{array}{l}\text { ț } \\
+ \\
+\end{array}$ & 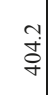 & \begin{tabular}{l|l}
$a$ & \\
0 \\
$o$ \\
$q$
\end{tabular} & 先 \\
\hline 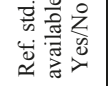 & $z_{1}$ & 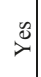 & $\stackrel{\circ}{z}$ & $\stackrel{\Delta}{*} \underbrace{\infty}$ & 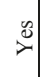 & 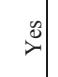 & 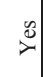 & & 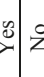 & & $\underbrace{\infty}_{\nu}$ & & z & & & & $\stackrel{\circ}{z}$ & 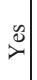 & 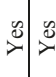 & $\stackrel{\circ}{z}$ & $z$ & 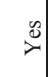 & z) & $z$ \\
\hline 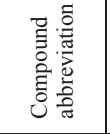 & 焉 & की & & 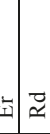 & $\bar{z}$ & $\begin{array}{l}z \\
\vdots\end{array}$ & $\begin{array}{l}z \\
\vdots \\
\vdots\end{array}$ & $=$ & है। & 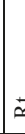 & & & & & & & 帘 & ฝँ & & & 至 & $\vec{z}$ & $\begin{array}{l}\text { 㝨 } \\
\text { 女 }\end{array}$ & \\
\hline$\widetilde{\underline{z}}$ & 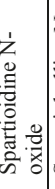 & 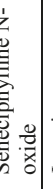 & : & \begin{tabular}{l|l}
0 \\
0
\end{tabular} & & 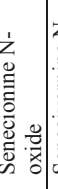 & 恶 & 1 & 要 & & & & & & & & 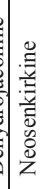 & 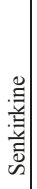 & 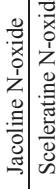 & 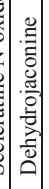 & \begin{tabular}{l|l}
$z$ & \\
0 \\
.
\end{tabular} & & 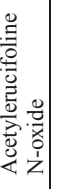 & \\
\hline
\end{tabular}




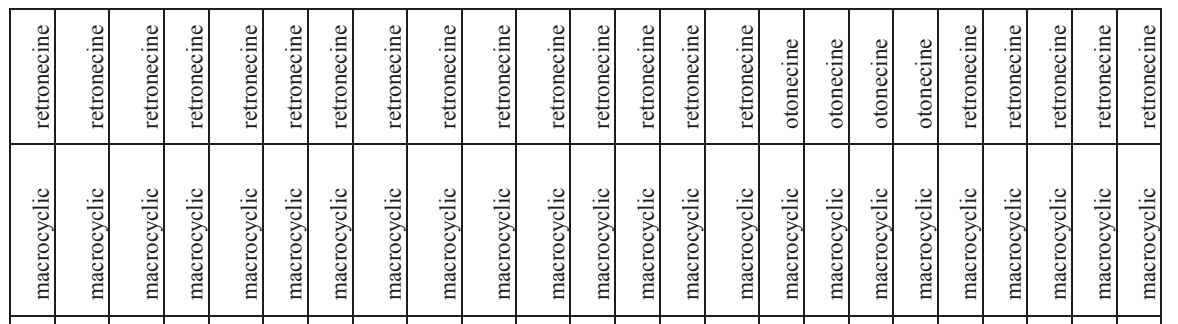

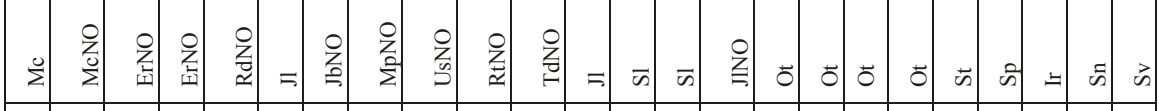

$\cdots \ldots \ldots \ldots \ldots \ldots \ldots \ldots$

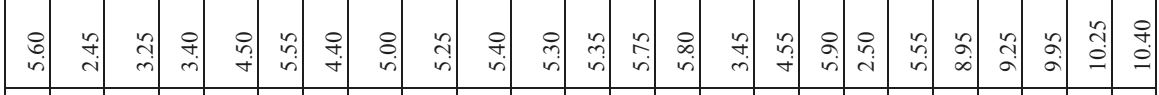
일

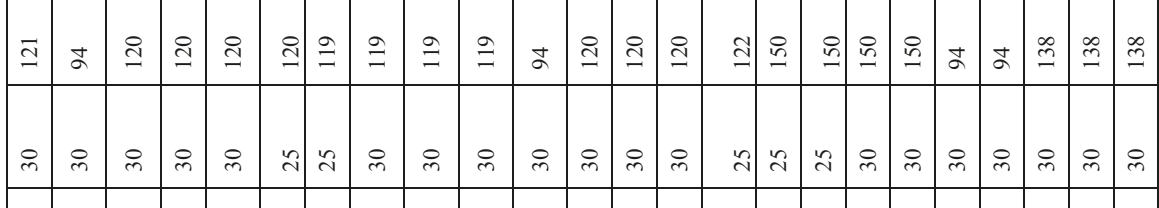

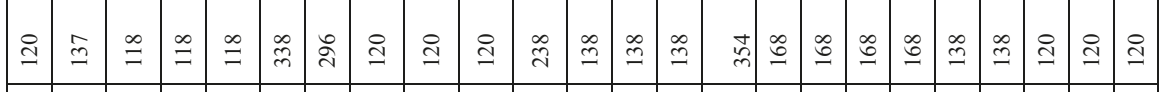

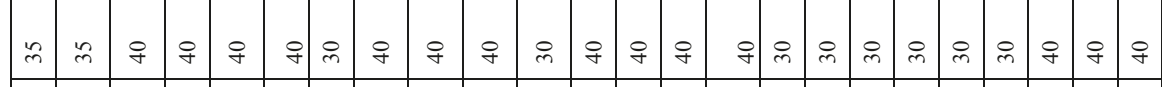

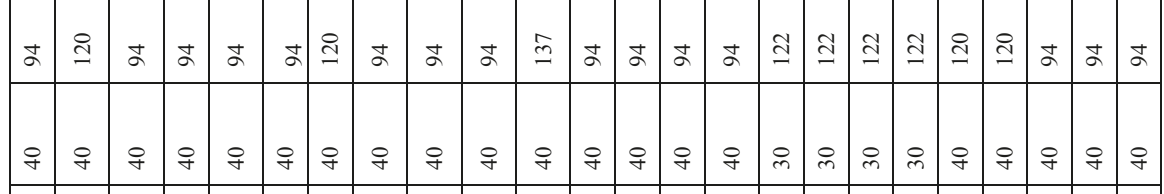

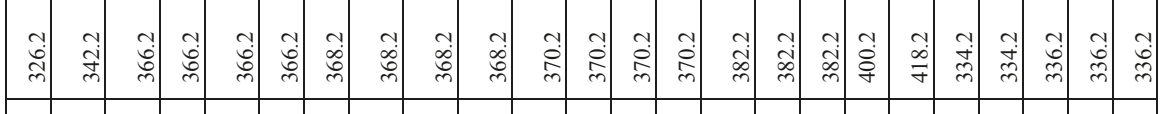

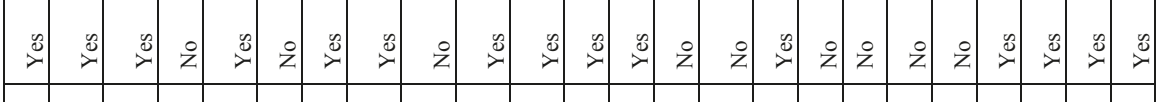

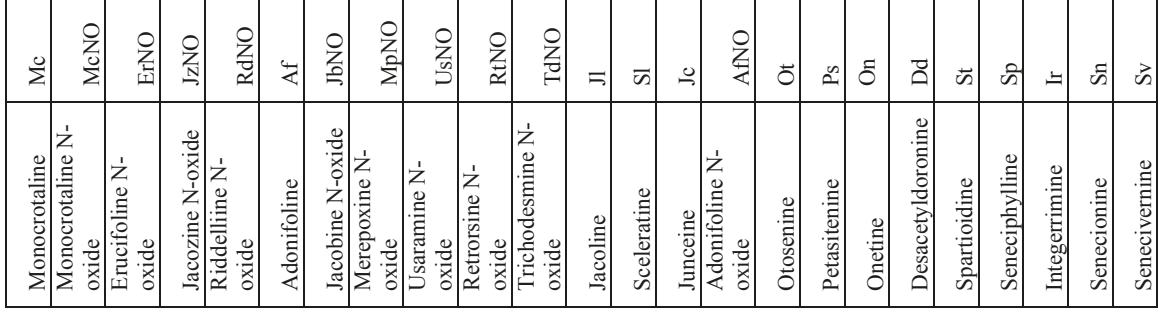




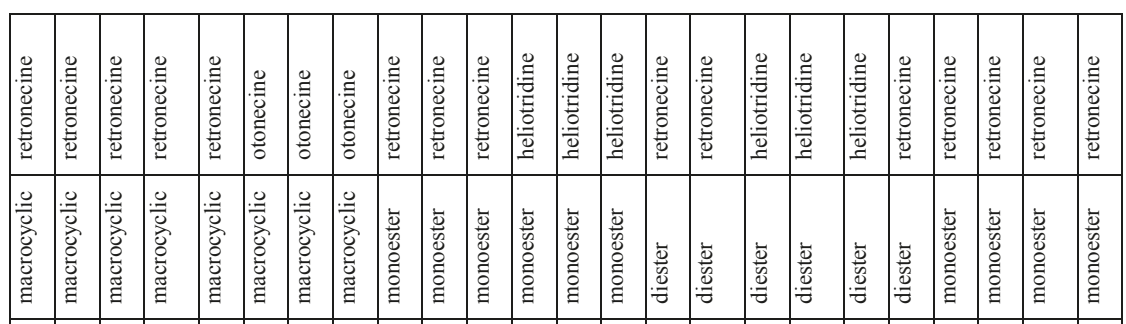

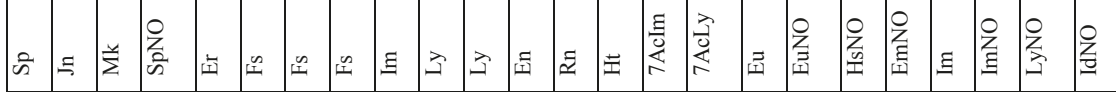

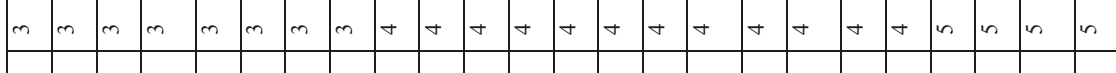

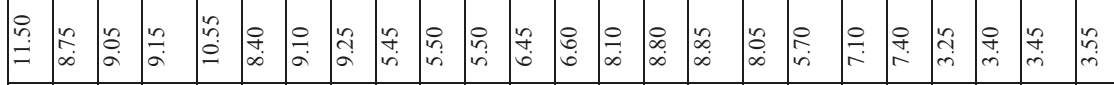

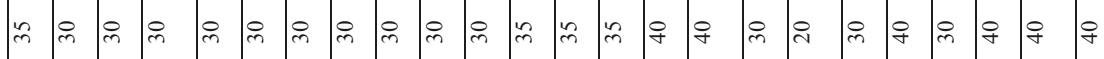

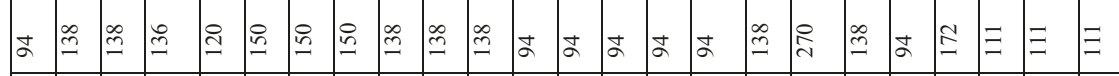

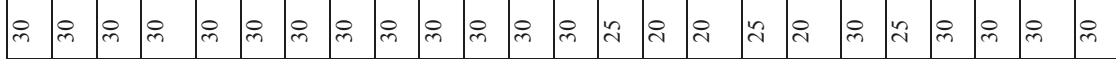

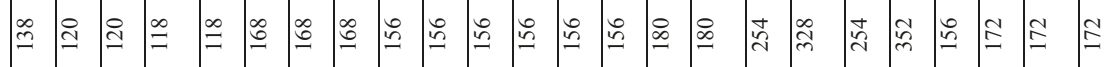

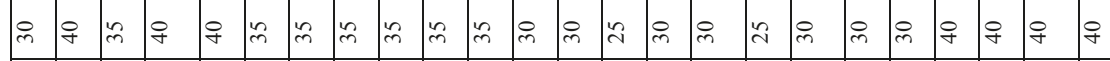

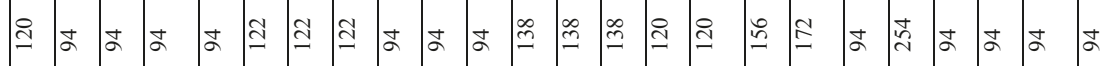

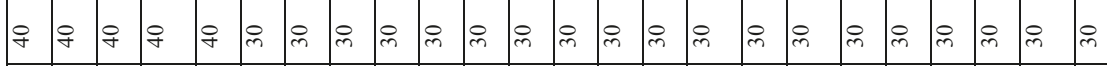

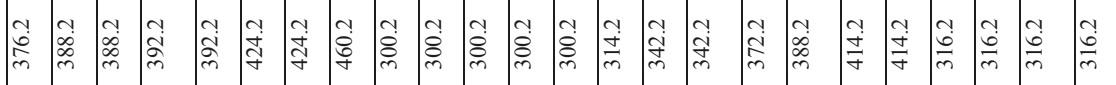

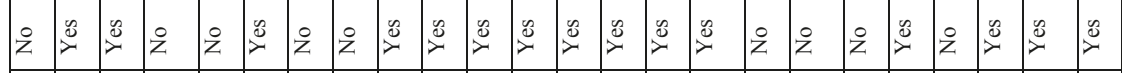

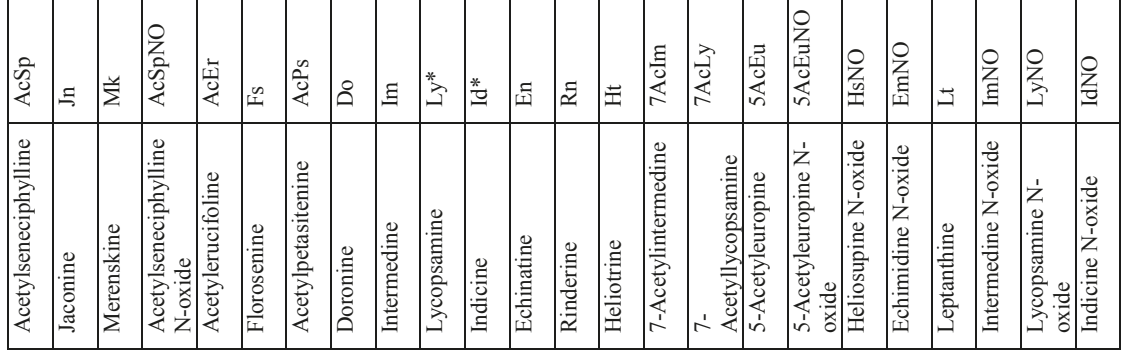




\begin{tabular}{|c|c|c|c|c|c|c|c|c|c|c|c|c|c|c|c|c|c|c|}
\hline 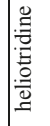 & 兽 & : & 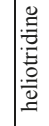 & 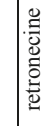 & : & & 苞 & 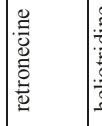 & 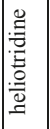 & 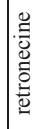 & 兽 & : & 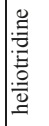 & 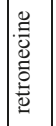 & 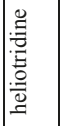 & 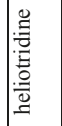 & 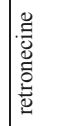 & : \\
\hline 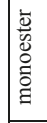 & 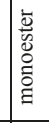 & 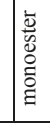 & 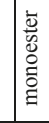 & 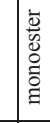 & 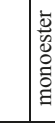 & 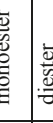 & 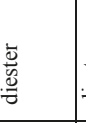 & 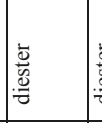 & 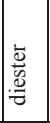 & 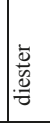 & 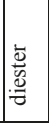 & 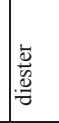 & 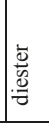 & 离 & 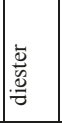 & 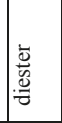 & 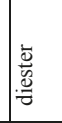 & 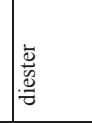 \\
\hline 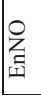 & 文 & 胥 & 函 & 亘 & 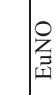 & 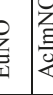 & 吾 & 目 & \pm & 臭 & د. & $\overbrace{0}^{0}$ & 正 & 吉 & 9 & 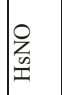 & 章 & 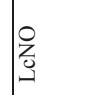 \\
\hline in & in & in & in & in & in & $n$ in & in & in & 6 & 6 & 6 & 0 & 6 & 0 & 6 & 0 & 0 & 0 \\
\hline 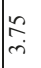 & 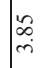 & $\begin{array}{l}8 \\
\text { in }\end{array}$ & $\frac{2}{6}$ & $\mid \begin{array}{l}\stackrel{\leftrightarrow}{u} \\
\stackrel{i}{*}\end{array}$ & $\begin{array}{l}n \\
n \\
m\end{array}$ & 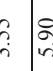 & $\begin{array}{l}8 \\
\text { in }\end{array}$ & in & 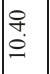 & $\begin{array}{l}n \\
n \\
\varrho\end{array}$ & 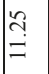 & 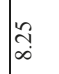 & 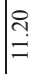 & $\stackrel{?}{=}$ & 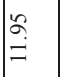 & $\begin{array}{l}\approx \\
\infty \\
\infty\end{array}$ & $\begin{array}{l}8 \\
\infty \\
\infty\end{array}$ & $\stackrel{2}{2}$ \\
\hline q & q & fo & m & & $\Re$ & $6=$ & ల & in & in & $\Re$ & i & o & i & ${ }_{m}$ & $\Re$ & in & q & in \\
\hline ta & \& & J & $\stackrel{2}{\curvearrowleft}$ & & 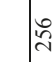 & 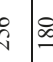 & $\stackrel{\infty}{\infty}$ & $\nsubseteq$ & & $\infty$ & 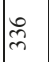 & I & స్ & $\infty$ & 芯 & $\stackrel{\infty}{\dddot{m}}$ & 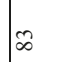 & 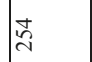 \\
\hline m & p & $\gtrsim$ & m & p & f & 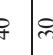 & ల) & in & ¿ & శิ & ¿ి & $\approx$ & ๙ิ & శి & $\approx$ & $\approx$ & $\approx$ & సి \\
\hline$\underline{\Sigma}$ & $\Xi$ & $\Xi$ & $\underset{m}{\infty}$ & $\underline{I}$ & $\Xi$ & $E \frac{\Delta}{N}$ & $\stackrel{\Delta}{N}$ & $\frac{7}{4}$ & సิ & సิ & సิ & 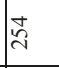 & సి & సิ & ల్ & 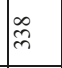 & $\underset{\infty}{\infty}$ & $\stackrel{\circ}{F}$ \\
\hline q & \& & 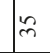 & 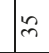 & in & in & $r$ & ¿ & in & $\Re$ & $\approx$ & $\approx$ & m & $\approx$ & $\approx$ & in & in & in & సి \\
\hline$\Xi$ & $\Xi$ & $\Xi$ & I & $\stackrel{\infty}{2}$ & $\underline{\Xi}$ & $\therefore \approx$ & $\hat{\mathrm{m}}$ & $\hat{\approx}$ & ‡ิ & ฮิ & 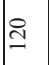 & $\stackrel{\infty}{\infty}$ & ฮิ & సి & 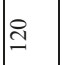 & 岕 & $\stackrel{ \pm}{\sim}$ & స̂. \\
\hline ஜ & p & లి & m & ஜ & ల & 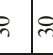 & 요 & 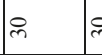 & 足 & in & o & on & on & p & in & in & i & i \\
\hline 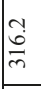 & $\frac{c}{3}$ & 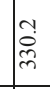 & ָૅ & స̃ & \ֶ. & 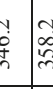 & $\begin{array}{l}\text { Na } \\
\infty \\
\infty \\
m \\
m\end{array}$ & 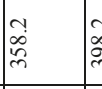 & $\begin{array}{l}c \\
\infty \\
\infty \\
\infty\end{array}$ & $\begin{array}{l}1 \\
\infty \\
\infty \\
m\end{array}$ & $\begin{array}{l}\mathcal{N} \\
\text { I } \\
\forall\end{array}$ & 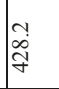 & 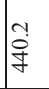 & 斿 & $\begin{array}{l}\text { y } \\
\text { ș } \\
\text { for }\end{array}$ & 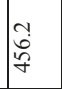 & 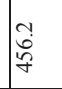 & 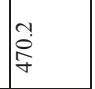 \\
\hline 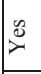 & $y^{2}$ & 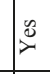 & $\underbrace{\bullet}$ & $\ddot{z}$ & 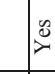 & 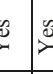 & $\nu^{y}$ & ¿্c & z & 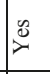 & 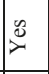 & 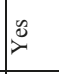 & $\AA$ & z & 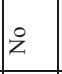 & $\gtreqless$ & z & $\check{z}$ \\
\hline 蛋 & 号 & 足 & ज्ञ & $Z_{\Xi}$ & 莤 & 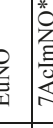 & 菊 & 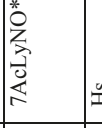 & $\approx$ & 节 & 3 & $\begin{array}{l}\text { 苂 } \\
\text { ذُ }\end{array}$ & $\begin{array}{l}\infty \\
\substack{0 \\
0 \\
m \\
m}\end{array}$ & 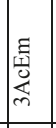 & \begin{tabular}{|c}
0 \\
0 \\
$\frac{0}{0}$ \\
in
\end{tabular} & 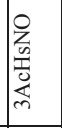 & 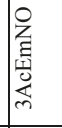 & 足 \\
\hline 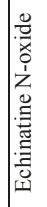 & 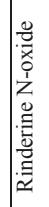 & 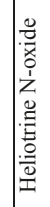 & 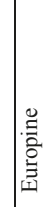 & 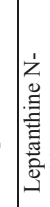 & 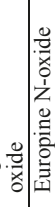 & 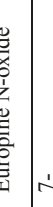 & 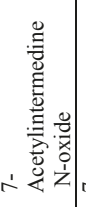 & 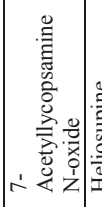 & 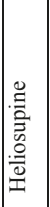 & 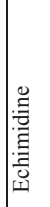 & 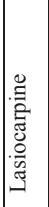 & 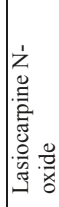 & 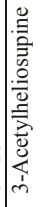 & 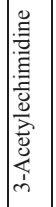 & & 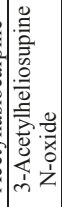 & 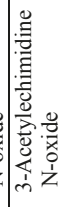 & 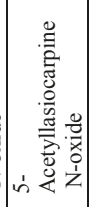 \\
\hline
\end{tabular}




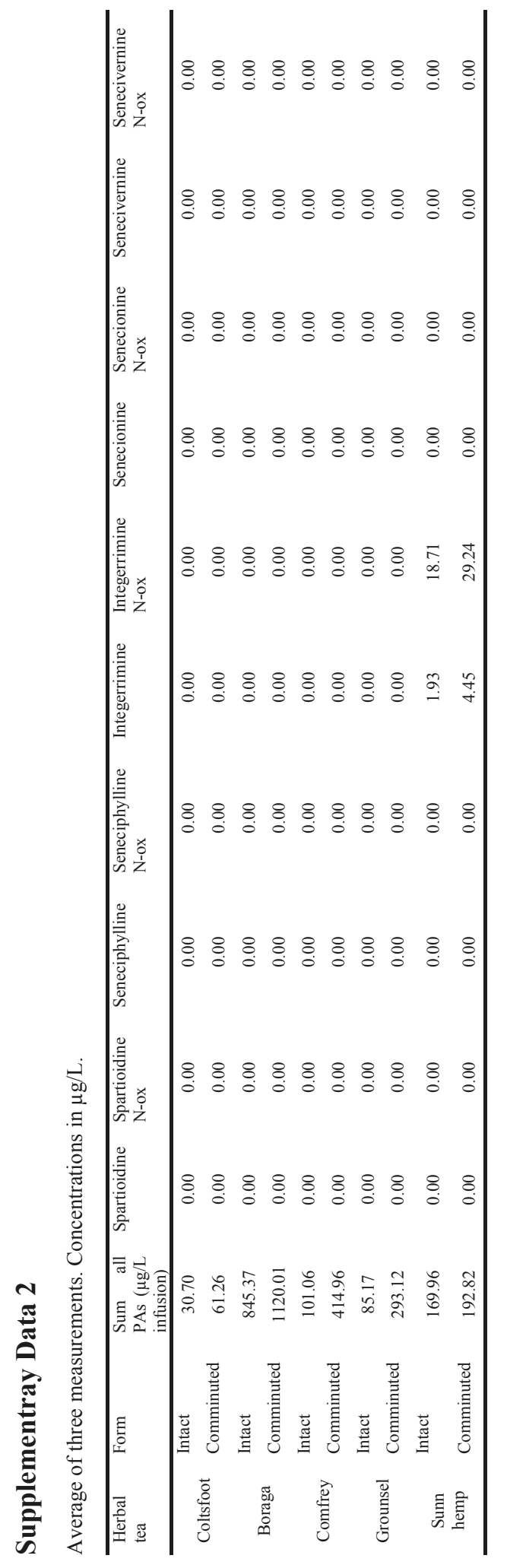




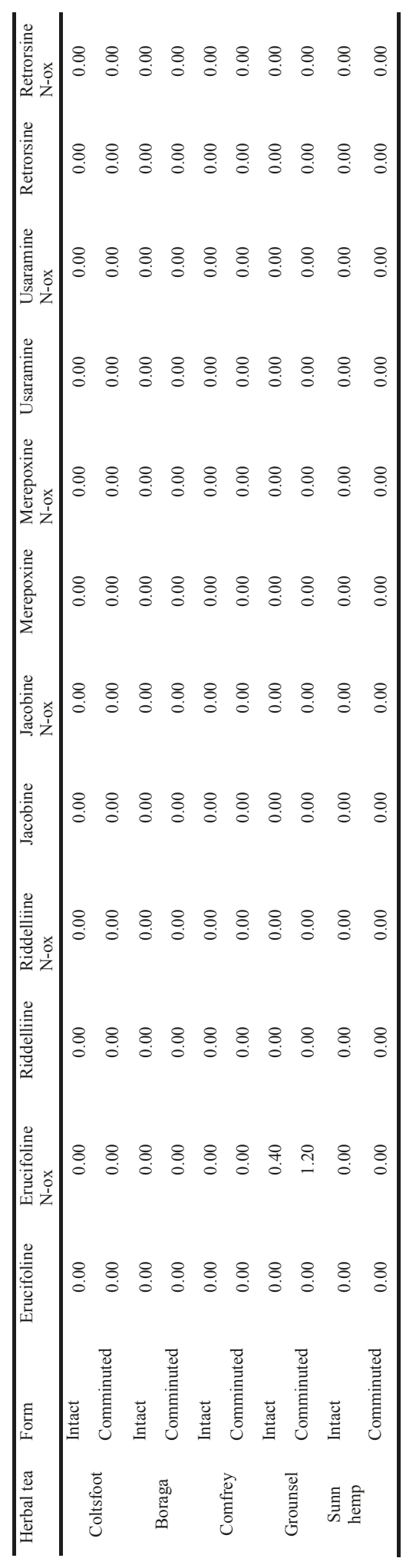




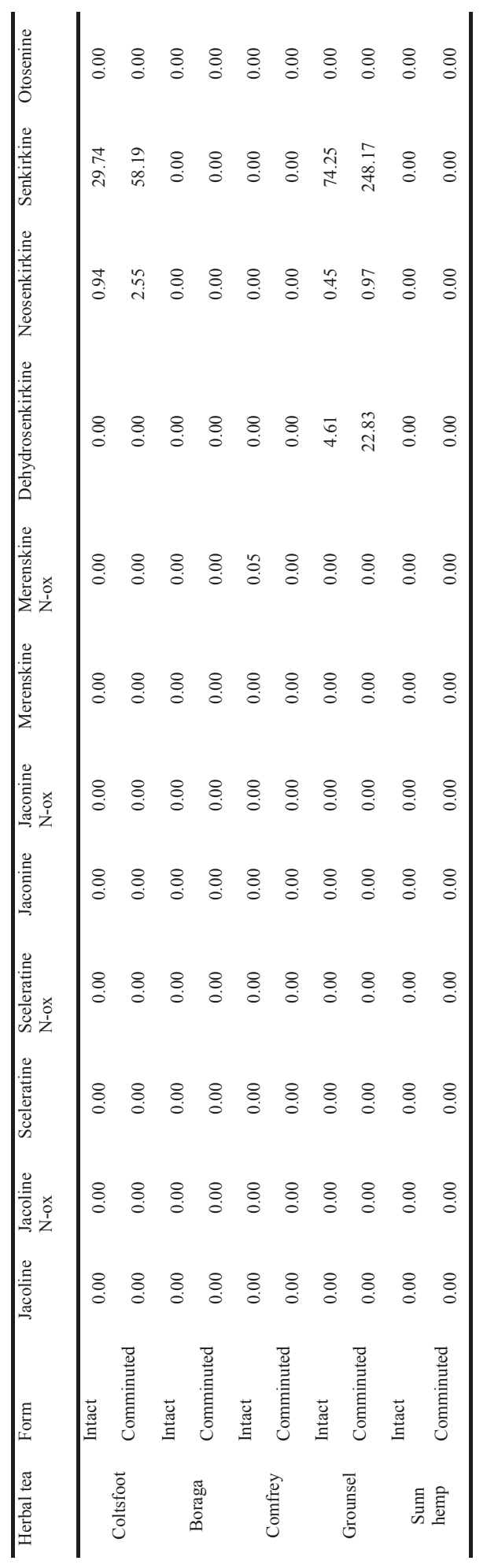




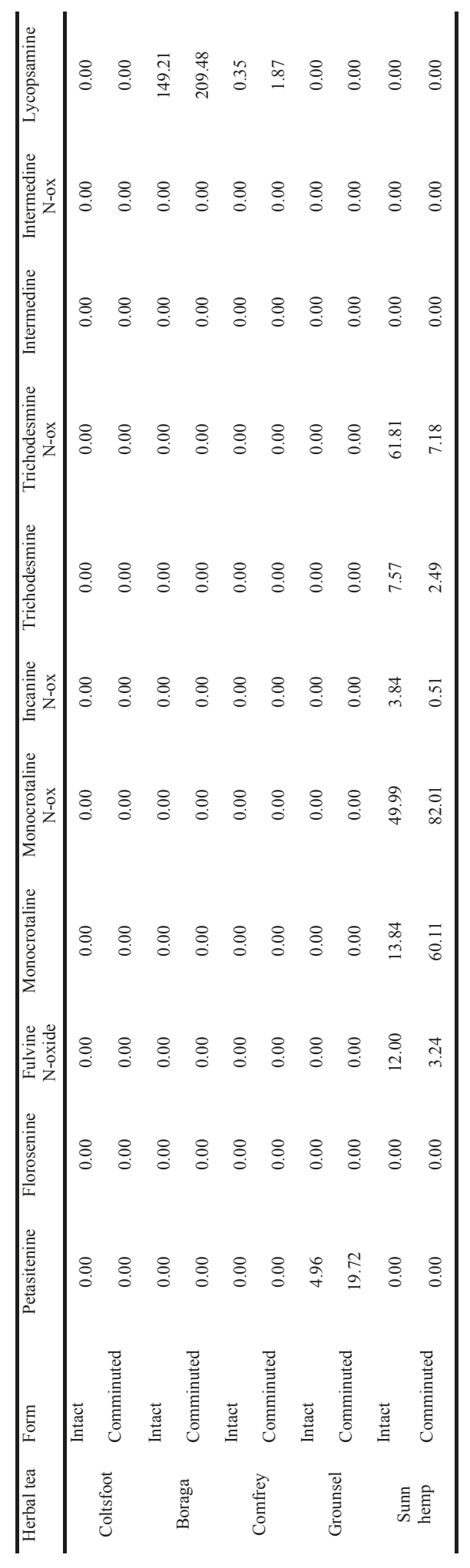




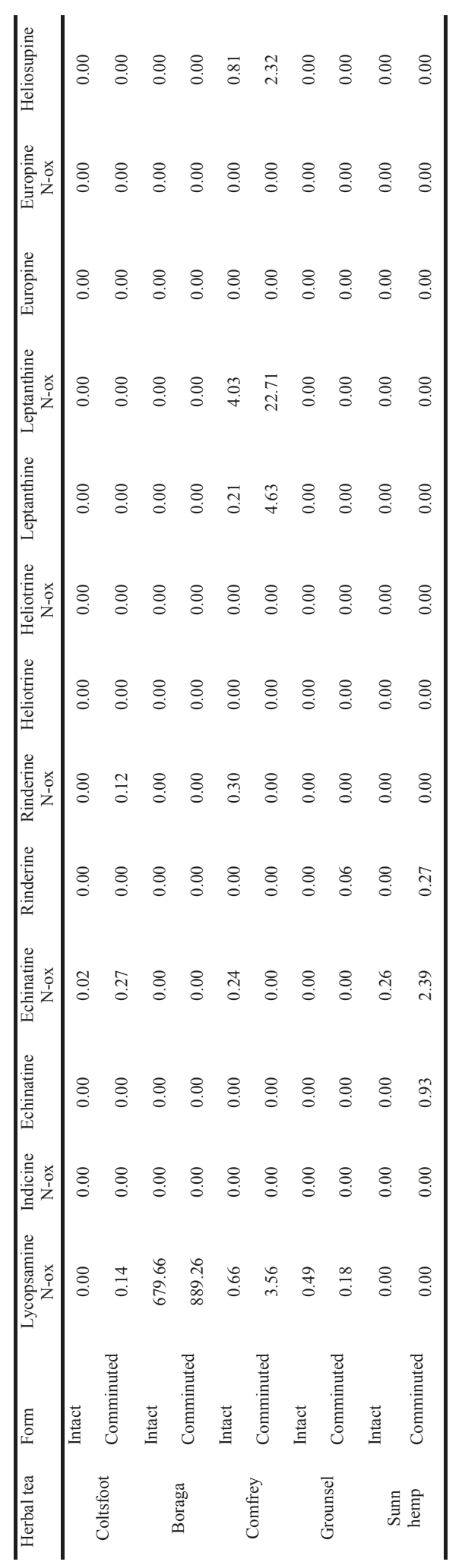

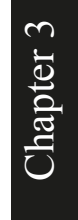




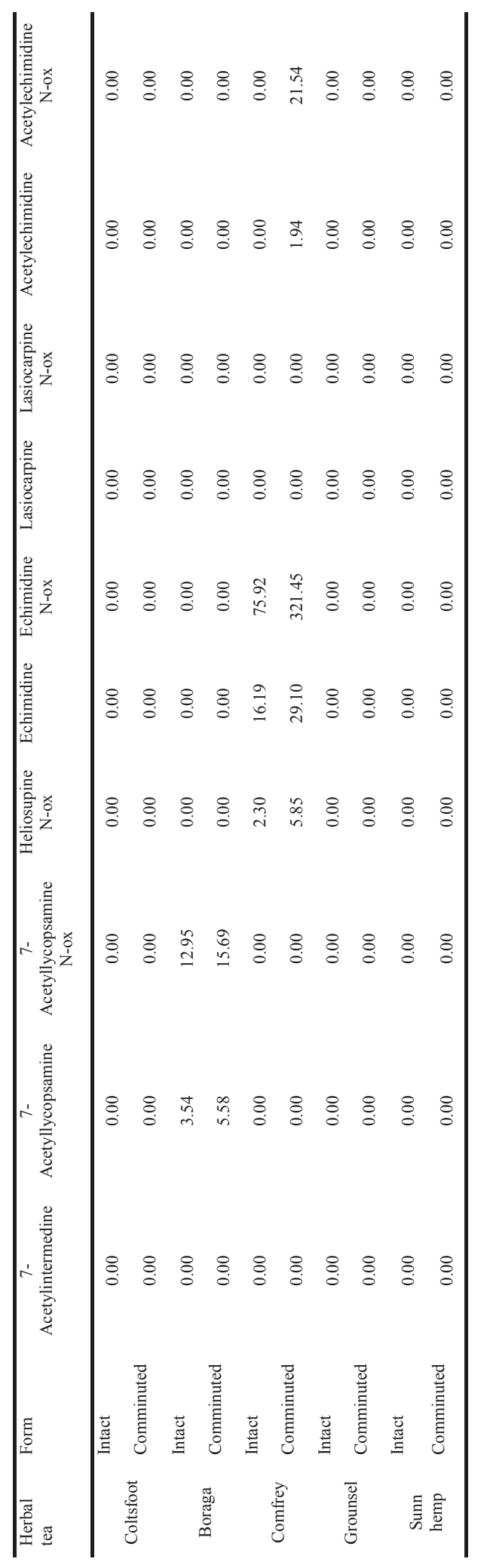




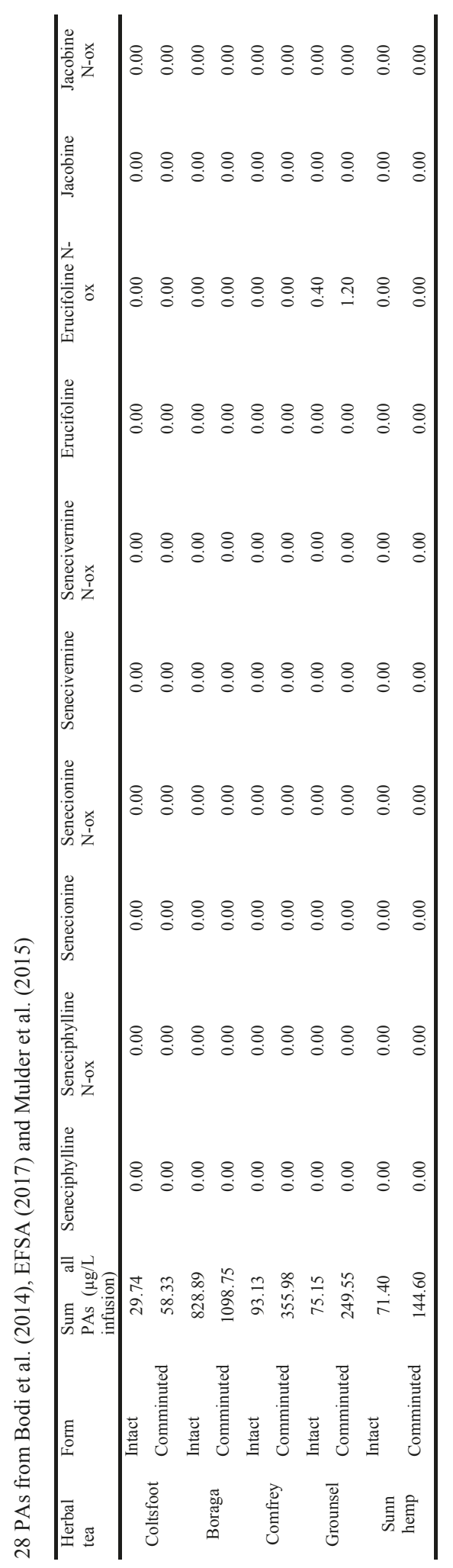




$$
\text { 自 }
$$




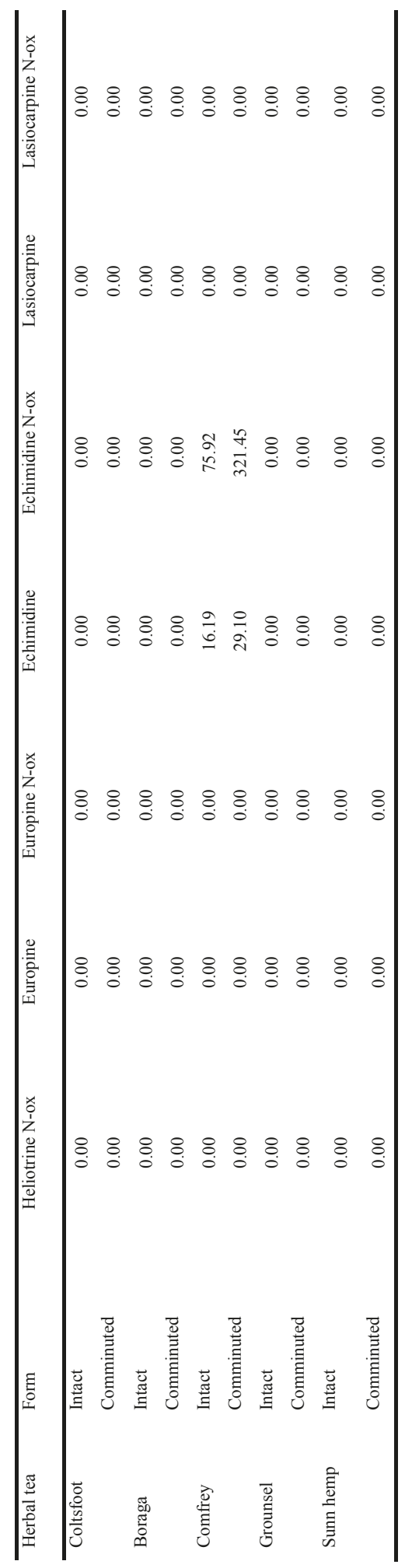




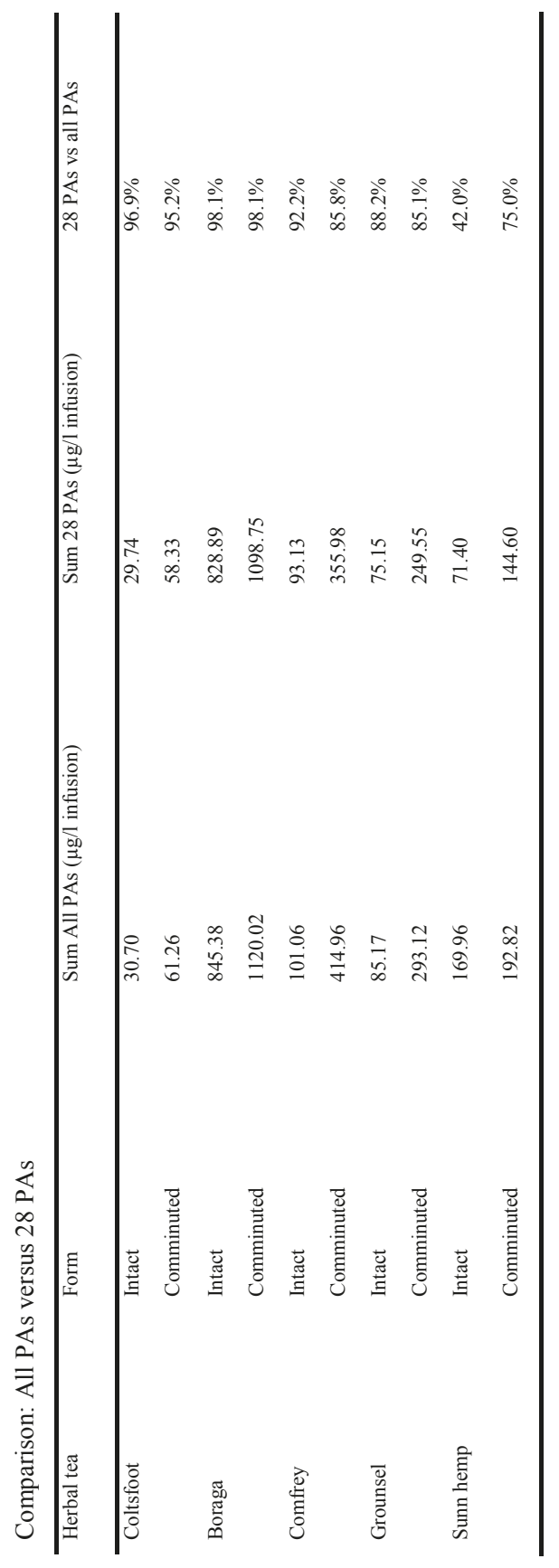




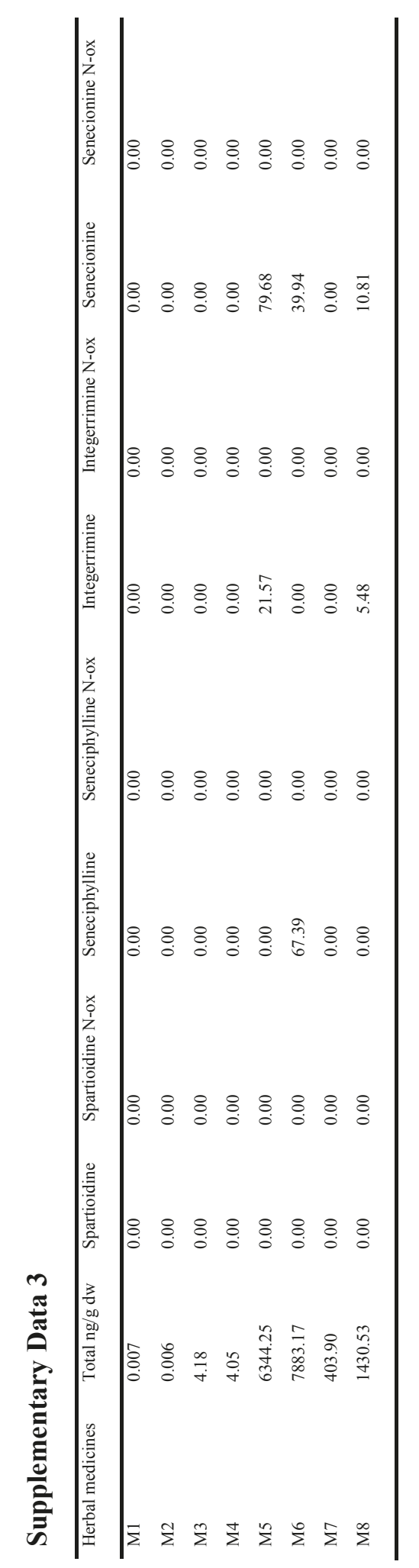




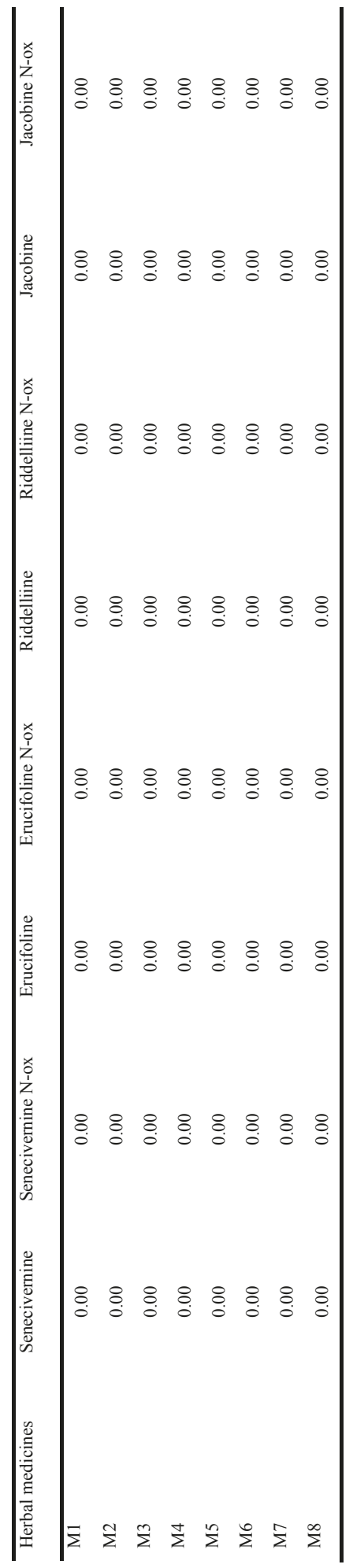



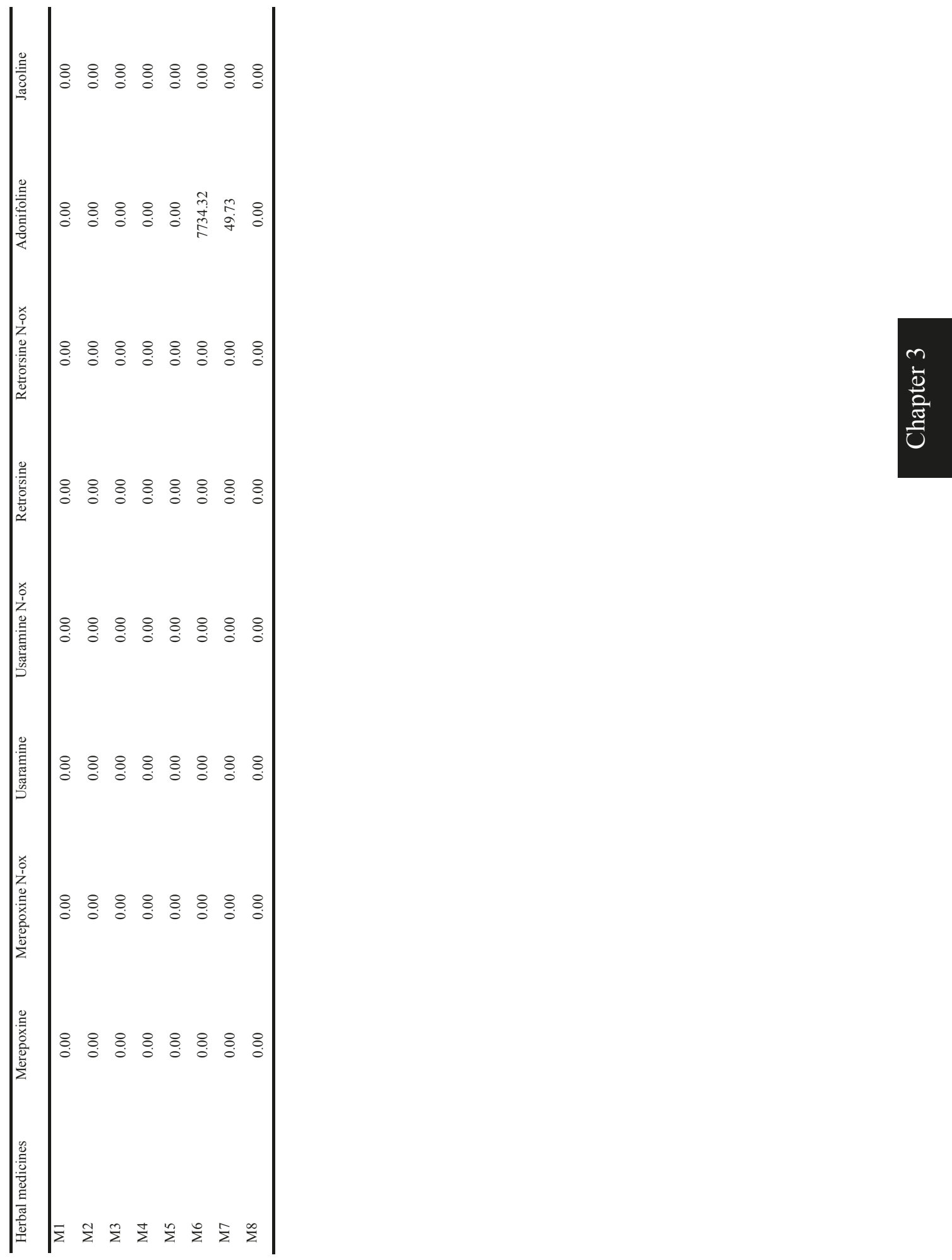


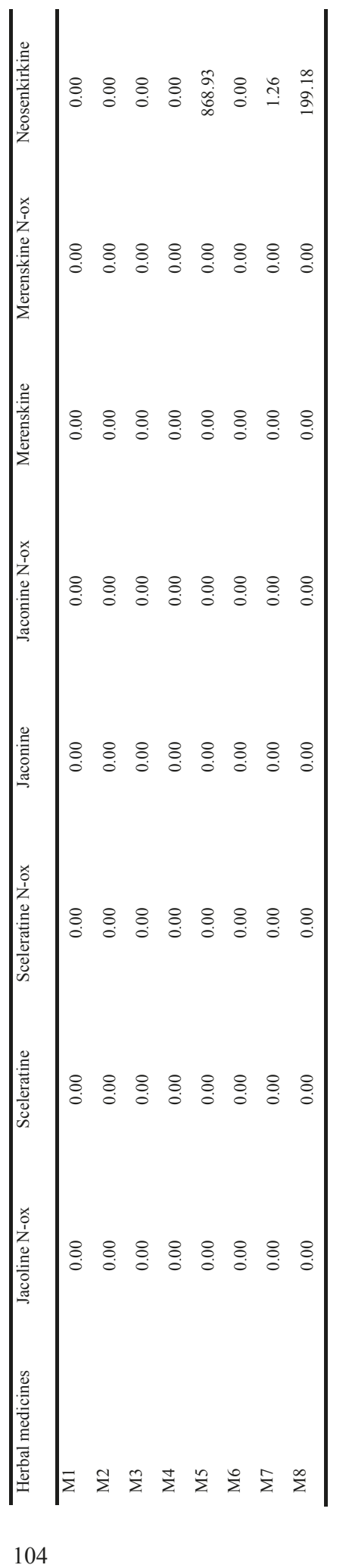




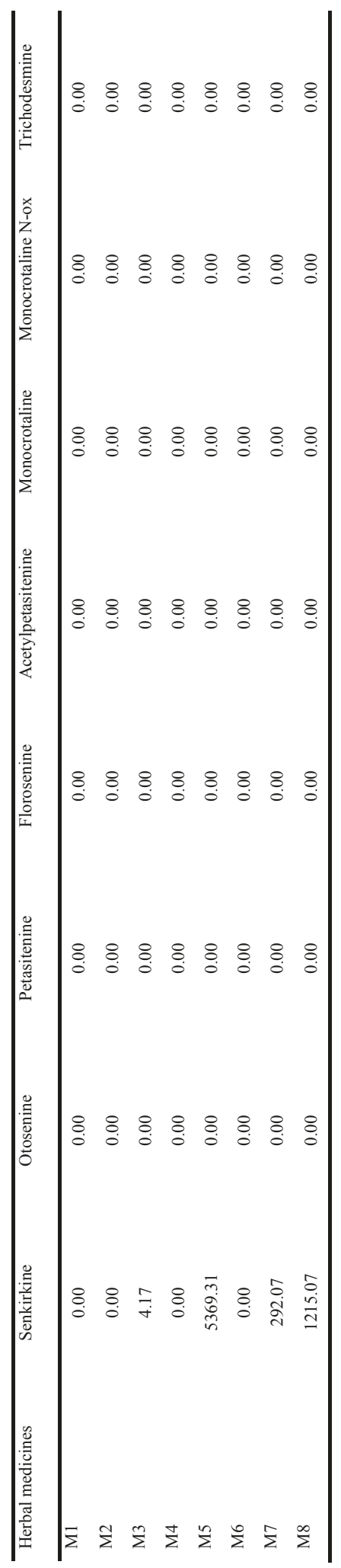




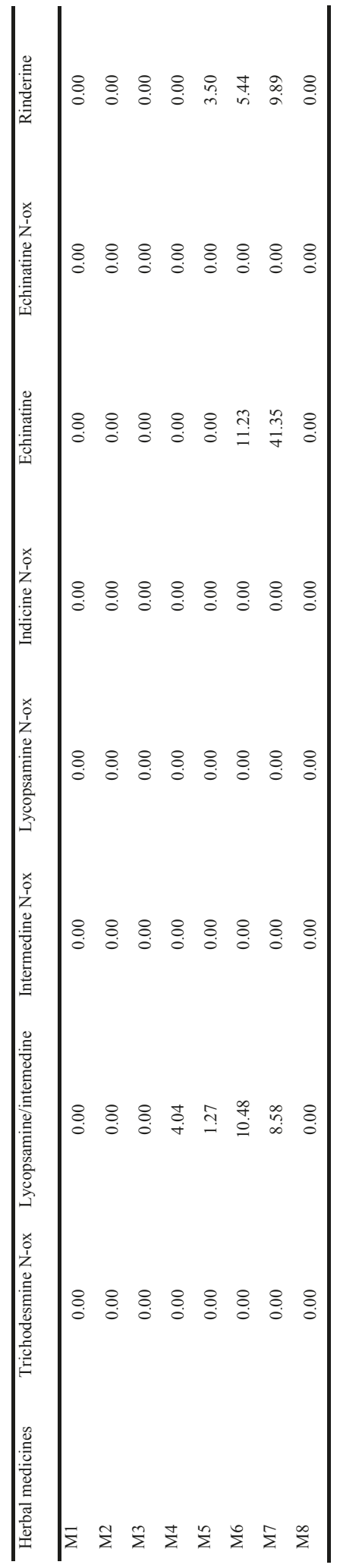




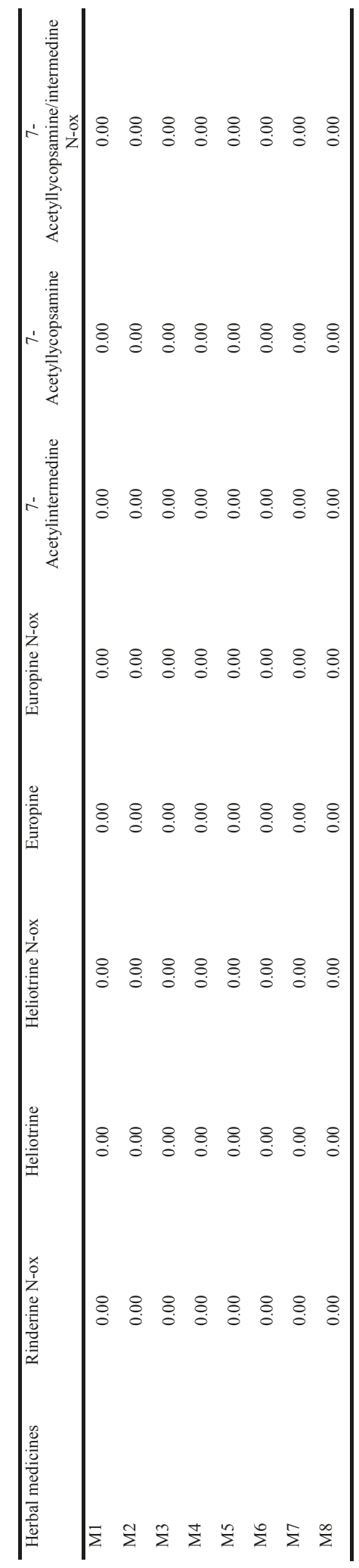




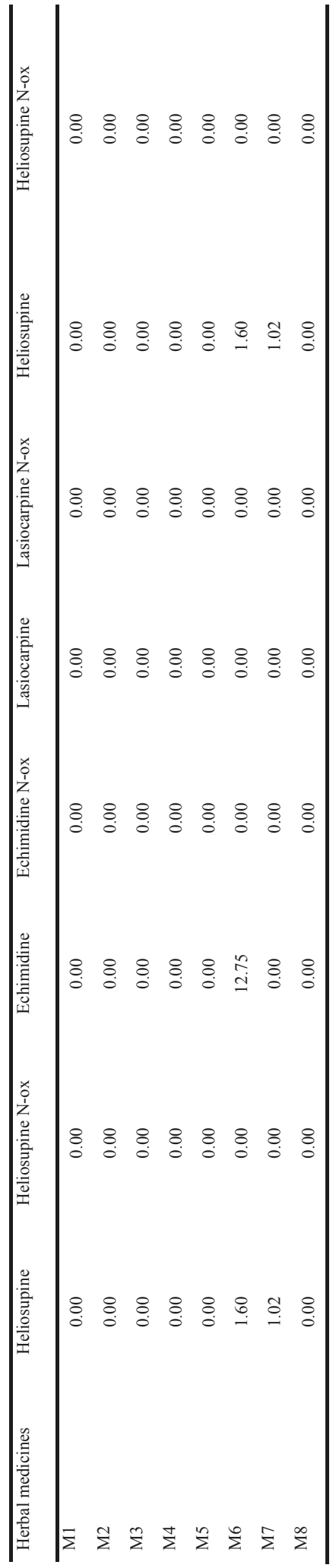




\section{Chapter 4}

\section{Use of physiologically based kinetic modelling-facilitated reverse dosimetry to convert in vitro cytotoxicity data to predicted in vivo liver toxicity of lasiocarpine and riddelliine in rat}

Lu Chen, Jia Ning, Jochem Louisse, Sebas Wesseling, Ivonne M.C.M. Rietjens

Published in: Food and Chemical Toxicology, (2018) 116, 216-226 


\begin{abstract}
Lasiocarpine and riddelliine are pyrrolizidine alkaloids (PAs) present in food and able to cause liver toxicity. The aim of this study was to investigate whether physiologically based kinetic (PBK) modelling-facilitated reverse dosimetry can adequately translate in vitro concentration-response curves for toxicity of lasiocarpine and riddelliine to in vivo liver toxicity data for the rat. To this purpose, PBK models were developed for lasiocarpine and riddelliine, and predicted blood concentrations were compared to available literature data to evaluate the models. Concentration-response curves obtained from in vitro cytotoxicity assays in primary rat hepatocytes were converted to in vivo dose-response curves from which points of departure (PoDs) were derived and that were compared to available literature data on in vivo liver toxicity. The results showed that the predicted PoDs fall well within the range of PoDs derived from available in vivo toxicity data. To conclude, this study shows the proofof-principle for a method to predict in vivo liver toxicity for PAs by an alternative testing strategy integrating in vitro cytotoxicity assays with in silico PBK modelling-facilitated reverse dosimetry. The approach may facilitate prediction of acute liver toxicity for the large number of PAs for which in vivo toxicity data are lacking.
\end{abstract}




\section{Introduction}

Pyrrolizidine alkaloids (PAs) are plant secondary metabolites (Fu et al., 2004; Mattocks, 1986) naturally occurring in several food items (EFSA, 2013; Wiedenfeld, 2011). To date, more than 660 PAs and PA $N$-oxides have been identified from more than 6000 plants (Wiedenfeld, 2013; Wiedenfeld and Edgar, 2011). PAs are a class of heterocyclic chemicals, and most PAs are derived from esters of basic alcohols, known as the necine bases. Especially the PAs with an 1,2-unsaturated necine base tend to be toxic (Mori et al., 1985). Of the four common necine bases encountered in PAs, including platynecine, retronecine, heliotridine and otonecine, especially the later three give rise to 1,2-unsaturated PAs that have been shown to be hepatotoxic, genotoxic and carcinogenic in rats and other experimental rodents (Chan, 1993; Chan et al., 2003; Hirono et al., 1979; Hirono et al., 1976; Hirono et al., 1977; Kuhara et al., 1980; NTP, 1978; Schoental, 1970; Shumaker et al., 1976). Human poisonings caused by intake of PA-containing plants have been reported in several countries (Kumana et al., 1985; Mohabbat et al., 1976; Prakash et al., 1999; Roulet et al., 1988; Sperl et al., 1995; Tandon et al., 1976). Due to the carcinogenicity of PAs, the International Agency for Research on Cancer (IARC) classified lasiocarpine and riddelliine in Group 2B, possibly carcinogenic to humans (IARC, 1976, 2002). In addition, the European Food Safety Authority (EFSA) recently stated that 1,2unsaturated PAs may act as carcinogens in humans based on the actual knowledge of metabolism, activation, DNA adduct-formation, genotoxicity and carcinogenicity (EFSA, 2011).

PAs need metabolic activation to form pyrrolic metabolites to exhibit their hepatotoxicity and genotoxicity (Chan et al., 2003; Chou et al., 2003; Fu et al., 2002, 2004; Lin et al., 2000; Mattocks, 1986; Miranda et al., 1991; Stegelmeier et al., 1999; Yan et al., 2002). In general, there are three principal metabolic pathways for the metabolism of 1,2-unsaturated PAs such as lasiocarpine (Figure 1). In the first pathway, the necine base is formed by hydrolysis of the ester group. For the second pathway, the necine base is N-oxidised to generate pyrrolizidine alkaloid N-oxide. In addition, the necine base can also be oxidised to form the corresponding reactive pyrrolic ester metabolite (Fu, 2016; Li et al., 2011). The generated pyrrolic ester can immediately bind to DNA and proteins in the liver resulting in DNA cross-links, DNA-protein cross-links, protein adducts and DNA adducts, that may induce hepatotoxicity and liver cancer (Bovee et al., 2015; Lin et al., 1998, 2000; Lin et al., 2011; White, 1976; Yan and Huxtable, 1995a, b).

In spite of the large number of PAs known at present, toxicity data on only a limited number of PAs are available. Among the 1,2-unsaturated PAs, lasiocarpine and riddelliine have been tested in two year rodent carcinogenicity bioassays by the National Toxicology Program (NTP), and were shown to cause liver carcinogenicity (NTP, 1978, 2003). However, of all these PAs, data on liver toxicity are only available for lasiocarpine (Dalefield et al., 2016; Jago, 1970; Nolan et al., 1966).

Given the large number of PAs and the limited number of PAs for which actual in vivo data on liver 
toxicity and carcinogenicity are available, it is of importance to investigate whether information on relative potency for hepatotoxicity of the different PAs can be obtained from alternative testing strategies reducing the need for a large number of animal experiments. In our previous work, we have shown that data on in vivo toxicity can be adequately predicted by translation of in vitro concentration-response curves for toxicity to in vivo dose-response curves for toxicity using so-called physiologically based kinetic (PBK) modelling-facilitated reverse dosimetry (Abdullah et al., 2016; Li et al., 2017; Louisse et al., 2015; Louisse et al., 2010; Strikwold et al., 2017). In this way in vivo doseresponse curves for developmental toxicity and kidney toxicity were defined (Abdullah et al., 2016; Li et al., 2017; Louisse et al., 2015; Louisse et al., 2010; Strikwold et al., 2017). The aim of the present study was to investigate whether PBK modelling-facilitated reverse dosimetry can adequately translate in vitro concentration-response curves for toxicity of PAs, to in vivo dose-response curves for liver toxicity for the rat. Riddelliine and lasiocarpine were used as model PAs because for these two PAs in vivo data on kinetics (Williams et al., 2002) and liver toxicity (Dalefield et al., 2016; Jago, 1970; Nolan et al., 1966), respectively, are available enabling evaluation of the PBK models and the toxicity predictions made.

To achieve the aim of this study, rat PBK models were developed for lasiocarpine and riddelliine, and concentration-response curves obtained from in vitro cytotoxicity studies with rat hepatocytes were translated into in vivo dose-response curves by using PBK modelling-facilitated reverse dosimetry from which PoDs for evaluation liver toxicity induced by lasiocarpine and riddelliine could be derived. For evaluating the performance of the PBK models, PBK model-predicted blood concentrations were compared with in vivo kinetic data. Because of the lack of any in vivo kinetic data of lasiocarpine, we also developed a PBK model for riddelliine in rat and mouse to enable examination of model performance based on available blood concentration values in the literature for this related PA. To evaluate the prediction of liver toxicity, PoDs derived from predicted liver toxicity induced by lasiocarpine were compared with available in vivo liver toxicity data of this PA. 


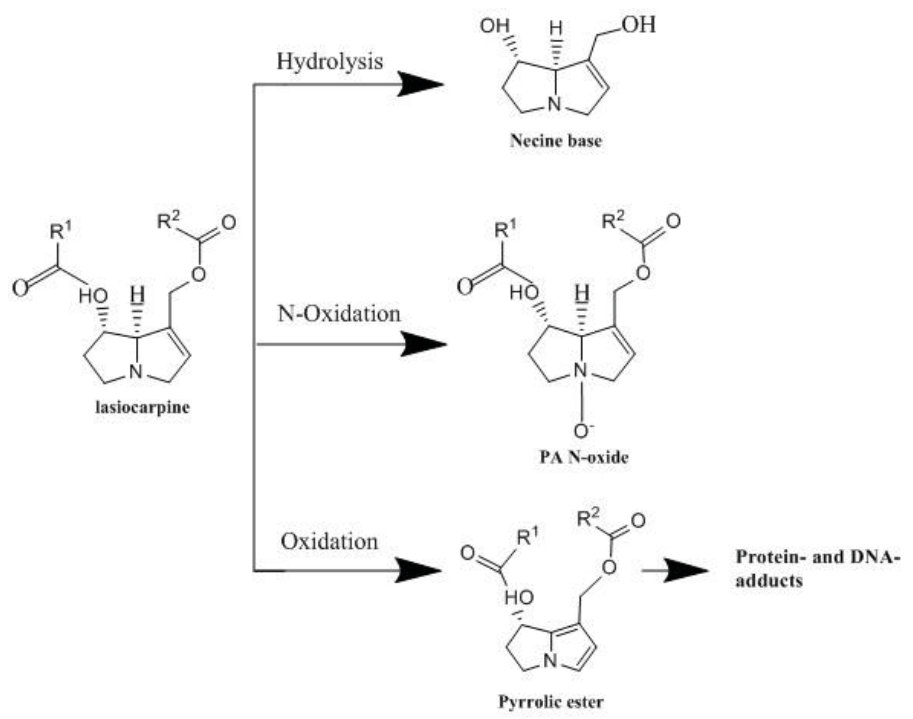

Fig. 1 The proposed pathways for metabolism of lasiocarpine, also relevant for riddelliine (Derived from Bovee et al., 2015; Fu, 2016; Li et al., 2011; Lin et al., 2011).

\section{Materials and Methods}

\section{Chemicals}

Lasiocarpine (> 97 \%) was purchased from PhytoLab (PhytoLab GmbH \& Co. KG, Germany), and riddelliine (90\%) was kindly provided by RIKILT Wageningen University \& Research (Wageningen, The Netherlands). The cryopreserved rat (Sprague-Dawley) hepatocytes, the thawing/plating supplement pack, the cell maintenance supplement pack, fetal bovine serum and Williams E Medium (WEM, A1217601) were purchased from ThermoFisher (Naarden, The Netherlands). Trypsin-EDTA was purchased from Gibco (Paisley, Scotland, UK). Dimethyl sulfoxide (DMSO) was obtained from Acros Organics (Geel, Belgium). Acetonitrile (UPLC/MS grade) was obtained from Biosolve (Valkenswaard, The Netherlands). Potassium hydrogen phosphate (K2HPO4) and trifluoroacetic acid (TFA) was purchased from Merck (Darmstadt, Germany). The reduced form of nicotinamide adenine dinucleotide phosphate (NADPH) was obtained from Sigma-Aldrich (Zwijndrecht, The Netherlands). Pooled liver microsomes from male Sprague-Dawley rats were obtained from Corning (Amsterdam, The Netherlands) and pooled liver microsomes from male CD-1 mice were purchased from Sigma Aldrich (Zwijndrecht, The Netherlands). Pooled intestinal microsomes from male Sprague-Dawley rats and male CD-1 mice were purchased from Xenotech (Lenexa, USA).

\section{General outline for PBK modelling-facilitated reverse dosimetry approach}

The combined in vitro PBK modelling approach to predict in vivo dose-response curves and a PoD for risk assessment using in vitro cytotoxicity data consisted of the following steps: (1) establishment 
of in vitro concentration-response curves for the toxicity of lasiocarpine and riddelliine in rat hepatocytes, (2) development of PBK models describing in vivo kinetics of lasiocarpine and riddelliine, using kinetic parameters defined based on in vitro assays using tissue fractions of rat and mouse, (3) evaluation of the PBK models against available literature data on blood levels and liver toxicity upon oral dosing of the PAs, (4) translation of the in vitro concentration-response curves for acute liver toxicity into in vivo dose-response curves for acute liver toxicity in rat using the PBK models, (5) BMD analysis on the predicted in vivo dose-response data to obtain a PoD for risk assessment, and (6) evaluation of the predicted PoD against available literature data.

\section{In vitro liver toxicity}

Rat hepatocytes were used to define the in vitro concentration-response curves for cytotoxicity. To this end rat hepatocytes were seeded at concentrations of $5 \times 10^{5}$ cells $/ \mathrm{ml}$ into 96 -well plates according to the supplier's protocol, and cultured in plating medium for 4-6 h. After incubation, medium was replaced by exposure medium (serum free), containing different concentrations of lasiocarpine or riddelliine ranging from 0 to $300 \mu \mathrm{M}$ (final concentrations), added from 200 times concentrated stock solutions in DMSO. After 23 hours treatment, $10 \mu \mathrm{MTT}(5 \mathrm{mg} / \mathrm{mL})$ was added and cells were incubated for an additional $1 \mathrm{~h}$. Cells were lysed and the MTT formazan crystals formed were dissolved by addition of $100 \mu \mathrm{DMSO}$, and the absorbance was measured at $562 \mathrm{~nm}$ and $620 \mathrm{~nm}$ using a microplate reader (SpectraMax M2, USA). Relative cell viability (\%) was calculated as (mean absorbance of sample/mean absorbance of vehicle control) $\times 100 \%$. Each concentration was tested in three replicates, and three independent experiments with 3 different batches of hepatocytes were carried out.

\section{Determination of fraction unbound (fub) of lasiocarpine and riddelliine in rat serum}

Since it is assumed that the toxicity is caused by the fraction unbound $\left(\mathrm{f}_{\mathrm{ub}}\right)$ of the chemical and since the $f_{u b}$ in vitro and in vivo differ, a correction for the differences in $f_{u b}$ needs to be made. The $f_{u b}$ of lasiocarpine and riddelliine in rat serum were determined by using rapid equilibrium dialysis (RED) (Waters et al., 2008). The RED device insert has two chambers, a blood and a buffer chamber. Briefly, a sample of $300 \mu \mathrm{l}$ of spiked rat serum containing $5 \mu \mathrm{M}$ of lasiocarpine or $50 \mu \mathrm{M}$ of riddelliine (final concentration, $0.5 \%$ DMSO) were added to the blood chamber, while $500 \mu$ PBS (containing 100 $\mathrm{mM}$ sodium phosphate and $150 \mathrm{mM}$ sodium chloride) were added to the buffer chamber. The device was sealed with tape and incubated at $37^{\circ} \mathrm{C}$ on a shaker at $250 \mathrm{rpm}$. After incubation for 5 hours the system reaches equilibrium (van Liempd et al., 2011) and $25 \mu \mathrm{L}$ of post-dialysis samples were collected from the blood and buffer chambers in separate tubes. Simultaneously, $25 \mu \mathrm{L}$ of corresponding buffer was added to the sample taken from the blood chamber and the same volume of corresponding rat serum was added to the sample taken from the buffer chamber. Then, $300 \mu \mathrm{L}$ cold precipitation buffer (90/10 acetonitrile/water) was added to both samples to precipitate the proteins 
and release the compound. The samples were put on ice for $30 \mathrm{~min}$ and subsequently centrifuged for $15 \mathrm{~min}$ at $15000 \mathrm{~g}$. Then, the supernatants were collected for UPLC analysis. By determining the compound concentration in each chamber, the $f_{u b}$ can be calculated with the following equation: $f_{u b}=$ (concentration in buffer chamber) / (concentration in blood chamber) (van Liempd et al., 2011; Waters et al., 2008). Then the effect concentration of lasiocarpine (LC) or riddelliine (RD) in rat serum $\left(\mathrm{C}_{\mathrm{LC} / \mathrm{RD}}\right.$, rat serum $)$ used for reverse dosimetry was corrected by the following equation: $\mathrm{C}_{\mathrm{LC}} / \mathrm{RD}$, rat serum $=$ $\mathrm{C}_{\mathrm{ub}}$, in vitro $/ \mathrm{f}_{\mathrm{ub}}$, rat serum, where $\mathrm{C}_{\mathrm{ub}}$, in vitro is the unbound concentration of lasiocarpine or riddelliine in the in vitro culture medium. Since the exposure medium for the rat hepatocytes was serum free, the concentration of lasiocarpine or riddelliine in the in vitro cytotoxicity assay was considered to be equal to the unbound concentration in rat serum.

\section{In vitro incubations of lasiocarpine or riddelliine to derive the kinetic parameters for the PBK model}

Microsomal incubations were performed with rat tissue fractions to determine kinetic parameter values for lasiocarpine and riddelliine clearance, using a substrate depletion approach. The incubation mixtures for lasiocarpine contained, in a final volume of $100 \mu \mathrm{l}$ (final concentrations): $0.1 \mathrm{M} \mathrm{K}_{2} \mathrm{HPO}_{4}$ $(\mathrm{pH} 7.4)$, pooled rat liver or intestinal microsomes $(0.04 \mathrm{mg}$ protein $/ \mathrm{ml})$, and lasiocarpine ranging from 0 to $200 \mu \mathrm{M}$ (added from 100 times concentrated stock solutions in DMSO). Under these conditions PA conversion was linear with time and the amount of protein added (data not shown). For riddelliine, the incubation mixtures contained in a final volume of $100 \mu \mathrm{l}$ (final concentrations): $0.1 \mathrm{M} \mathrm{K}_{2} \mathrm{HPO}_{4}$ $(\mathrm{pH} 7.4)$, pooled rat liver or intestinal microsomes $(0.5 \mathrm{mg}$ protein $/ \mathrm{ml})$, and riddelliine ranging from 0 to $400 \mu \mathrm{M}$ (added from 100 times concentrated stock solutions in DMSO). Under these conditions PA conversion was linear with time and the amount of protein added (data not shown). After 5 min of preincubation in a shaking water bath at $37{ }^{\circ} \mathrm{C}$, the reactions were started by the addition of $2 \mathrm{mM}$ NADPH. The reactions were carried out for $30 \mathrm{~min}$ with liver microsomes and $2 \mathrm{~h}$ with intestinal microsomes. Control incubations were performed without NADPH. The incubations were terminated by the addition of $25 \mu \mathrm{l}$ ice-cold acetonitrile followed by centrifugation at $16000 \mathrm{~g}$ for $5 \mathrm{~min}$ before analyse of the supernatant by UPLC (Waters Acquity). The incubation conditions for metabolism of riddelliine by pooled mouse liver and intestinal microsomes were performed in a similar way. The clearance of lasiocarpine and riddelline in lung or kidney was determined using either lung or kidney microsomes of rat or mouse, by following the same incubation procedure for intestinal microsomes using final microsomal concentrations of $0.04 \mathrm{mg} / \mathrm{mL}$ for rat and $0.5 \mathrm{mg} / \mathrm{mL}$ for mouse.

From each incubation, $3.5 \mu \mathrm{l}$ of supernatant were injected into the UPLC (Waters Acquity) equipped with a Waters BEH C18 $1.7 \mathrm{~mm}$ column, $2.1 \times 50 \mathrm{~mm}$ (Waters, Ireland). A gradient was made with ultra-pure water containing $0.1 \%(\mathrm{v} / \mathrm{v})$ TFA as solvent A and $100 \%$ acetonitrile as solvent $\mathrm{B}$. The flow rate was set to $0.6 \mathrm{ml} / \mathrm{min}$. The starting condition was 100:0 (A:B), changing to $50: 50$ from 0 to 2 
$\mathrm{min}$, then to 0:100 from 2 to $5 \mathrm{~min}$, then to 100:0 from 5 to $8 \mathrm{~min}$. Detection was carried out with a photodiode array detector (Waters, Milford, MA, USA) at a wavelength of $220 \mathrm{~nm}$. The amount of substrate depletion of lasiocarpine or riddelliine was quantified as the amount detected in the control incubations performed without the respective cofactor NADPH minus the amount detected in the incubations with NADPH using a calibration curve made with available reference compounds. The data for the rate of depletion of lasiocarpine and riddelliine with increasing lasiocarpine or riddelliine concentrations were fitted to the standard Michaelis-Menten equation:

$V=\operatorname{Vmax} *[S] /(\mathrm{Km}+[S])$

with [S] being the substrate concentration, $\mathrm{V}_{\max }$ being the apparent maximum velocity, and $\mathrm{K}_{\mathrm{m}}$ being the apparent Michaelis-Menten constant. This was done with GraphPad Prism, 5.0 software (San Diego, CA, USA).

\section{PBK models for rat and mouse}

A schematic diagram of the structure of the PBK models developed for rat and mouse is shown in Fig.

2. The models include separate compartments for liver and intestine (as metabolizing compartments), fat, arterial blood, venous blood, rapidly perfused and slowly perfused tissue. Lasiocarpine and riddelliine were assumed to be taken up from the gastrointestinal tract following first order kinetics and absorbed directly by the intestine. No absorption rate constants $(\mathrm{Ka})$ are available for lasiocarpine and riddelliine, but for the PA adonifoline, a Ka of $0.6 / \mathrm{h}$ has been reported (Wang et al., 2011). The $\mathrm{Ka}$ of lasiocarpine and riddelliine was estimated based on two approaches. In the first approach, the Ka was estimated by relating the Ka values of the PAs to the permeability coefficients ( $P_{\text {app }}$ values) of the PAs as predicted by Qikprop (Schrödinger, trial version, Germany). The $P_{\text {app }}$ values of adonifoline, lasciocarpine and riddelliine as derived from Qikprop amount to 241, 542 and $107 \mathrm{~nm} / \mathrm{s}$ for adonifoline, lasiocarpine and riddelliine, respectively. The Ka values for lasioicarpine and riddelliine were calculated relative to adonifoline as the standard compound $\left(P_{\text {app LC/RD }} / \mathrm{Ka}_{\mathrm{LC} / \mathrm{RD}}=P_{\text {app adonifoline }} /\right.$ 0.6 ). Based on the $P_{\text {app }}$ for lasicoarpine and riddelliine of $542 \mathrm{~nm} / \mathrm{s}$ and $107 \mathrm{~nm} / \mathrm{s}$, Ka values for lasiocarpine and riddelliine were calculated to amount to $1.35 / \mathrm{h}$ and $0.27 / \mathrm{h}$, respectively. Ka values for lasiocarpjne and riddelliine were also estimated based on $P_{\text {app }}$ values obtained from the correlation between Caco-2 permeation and molecular properties described by Hou et al. (Hou et al., 2004) reflected by the equation $\log P_{\text {app }}=-5.469+0.236 \log \mathrm{P}$. The $\log \mathrm{P}$ values for lasiocarpine, riddelliine and adonifoline were obtained using the ChemBio 3D 2010 software (CambrigeSoft, USA) and amounted to $0.48,-0.26$ and -1.49 , respectively. Based on these $\log \mathrm{P}$ values, the corresponding $\log$ $P_{\text {app }}$ values were calculated to be -5.35 for lasiocarpine, -5.53 for riddelliine and -5.82 for adonifoline. Then, the relative Ka values for lasiocarpine or riddelliine were calculated relative to adonifoline as the standard compound as described above $\left(\log P_{\text {app LC/RD }} / \mathrm{Ka}_{\mathrm{LC} / \mathrm{RD}}=\log P_{\text {app }}\right.$ adonifoline / 0.6). 
The Ka values thus obtained for lasiocarpine and riddelliine were $1.75 / \mathrm{h}$ and $1.17 / \mathrm{h}$, respectively. In this study, we calculated the average Ka values for lasiocarpine and riddelliine from those two methods, which amounted to $1.55 / \mathrm{h}$ and $0.72 / \mathrm{h}$, respectively. Physiological parameter values for the model, such as for tissue volumes and blood flows, were obtained from literature (Brown et al., 1997). The tissue/blood partition coefficients applied in the rat model were calculated based on the method reported by DeJongh et al. (DeJongh et al., 1997). All physiological parameters and partition coefficients used in the rat PBK models are presented in Table 1. For evaluation of the generic PBK models developed in the present study, we also developed a mouse PBK model for riddelliine, because for riddelliine also kinetic data in mice were available for model evaluation (Williams et al., 2002). The physiological parameters for the mouse PBK model were taken from literature (Brown et al., 1997) and the tissue/blood partition coefficients were assumed to be the same as in rat. These values used in this mouse PBK model are also presented in Table 1. On the basis of data obtained in the in vitro incubations with liver and intestinal fractions with lasiocarpine and riddelline, the conversion of lasiocarpine and riddelliine were described in the liver and intestine. The $\mathrm{V}_{\max }$ values for conversion of lasiocarpine or riddelliine by rat or mouse liver and intestinal microsomal fractions were scaled accordingly using an estimated microsomal protein yield of $35 \mathrm{mg} / \mathrm{g}$ liver (Medinsky et al., 1994) and $20.6 \mathrm{mg} / \mathrm{g}$ small intestine (Cubitt et al., 2009), respectively as scaling factors. The in vivo $\mathrm{K}_{\mathrm{m}}$ values were assumed to equal the in vitro $\mathrm{K}_{\mathrm{m}}$ values.

In the present study it was assumed that clearance of the PAs fully depends on their metabolic conversion and that excretion of the parent compound in its unmodified form does not add significantly to the systemic clearance. This assumption is supported by data reported by Bull et al. (1968) who delivered lasiocarpine to rats through ip injection, after which only 1-1.5\% of total lasiocarpine was found in unmodified form in the urine (Bull et al., 1968), so excretion of the parent compound was not included in the current PBK model. The set of differential equations describing the mass balance equations can be found in the supplementary data. The PBK model equations were coded and numerically integrated in Berkeley Madonna (version 8.3.18, UC Berkeley, CA, USA) using Rosenbrock's algorithms for solving stiff systems. 


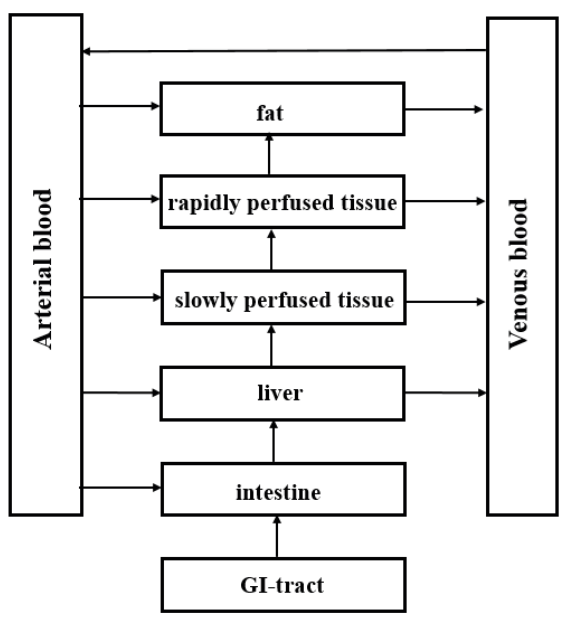

Fig. 2 Schematic diagram of the generic PBK model for lasiocarpine and riddelliine in rat and mouse. 
Table 1 Parameters used in the rat or mouse PBK models for lasiocarpine and riddelliine.

\begin{tabular}{|c|c|c|}
\hline \multirow[t]{2}{*}{ Parameters } & \multicolumn{2}{|c|}{ Values } \\
\hline & Rat & Mouse \\
\hline \multicolumn{3}{|l|}{ Physiological parameters $^{\mathrm{a}}$} \\
\hline Body weight (kg) & 0.25 & 0.0025 \\
\hline \multicolumn{3}{|l|}{ Tissue volumes ( $\%$ body weight) } \\
\hline Liver & 3.4 & 5.5 \\
\hline Fat & 7.0 & 10 \\
\hline Small intestine & 1.4 & 2.5 \\
\hline Richly perfused tissue & 4.2 & 1 \\
\hline Slowly perfused tissue & 75 & 72 \\
\hline Blood & 7.4 & 4.9 \\
\hline Cardiac output $\left(\mathrm{L} \cdot \mathrm{h}^{-1} \cdot \mathrm{kg} \mathrm{bw}^{-0.74}\right)$ & 15.0 & 15.0 \\
\hline \multicolumn{3}{|l|}{ Blood flow to tissue ( $\%$ cardiac output) } \\
\hline Liver & 13.2 & 6.7 \\
\hline Fat & 7 & 9 \\
\hline Small intestine & 11.8 & 18.3 \\
\hline Richly perfused tissue & 51 & 51 \\
\hline Slowly perfused tissue & 17 & 15 \\
\hline \multicolumn{3}{|l|}{ Physicochemical parameters ${ }^{b}$} \\
\hline \multicolumn{3}{|l|}{ Tissue/blood partition coefficients } \\
\hline \multicolumn{3}{|l|}{ Lasiocarpine } \\
\hline Liver & 88 & 88 \\
\hline Fat & 244 & 244 \\
\hline Small intestine & 88 & 88 \\
\hline Richly perfused tissue & 88 & 88 \\
\hline Slowly perfused tissue & 48 & 48 \\
\hline \multicolumn{3}{|l|}{ Riddelliine } \\
\hline Liver & 77 & 77 \\
\hline Fat & 44 & 44 \\
\hline Small intestine & 77 & 77 \\
\hline Richly perfused tissue & 77 & 77 \\
\hline Slowly perfused tissue & 43 & 43 \\
\hline
\end{tabular}

a (Brown et al., 1997)

${ }^{\mathrm{b}}$ Derived using the approach described by DeJongh et al. (1997) 


\section{PBK model evaluation}

Normalized sensitivity coefficients (SC) were determined to identify parameters that largely influence the prediction of the maximal blood concentrations $\left(\mathrm{C}_{\max }\right)$ in liver blood by the PBK model, using the equation:

$\mathrm{SC}=\left(\left(\mathrm{C}^{\prime}-\mathrm{C}\right) /\left(P^{\prime}-P\right)\right) *(P / C)$

where $\mathrm{P}$ and $\mathrm{P}^{\prime}$ are the initial and modified parameter values respectively, and $\mathrm{C}$ and $\mathrm{C}^{\prime}$ are the initial and modified values of the model output resulting from an increase in parameter value, respectively (Evans and Andersen, 2000). A 5\% increase in parameter value was chosen to analyse the effect of a change in a parameter. The sensitivity analysis was conducted at an oral dose of 0.008 and 0.144 $\mu \mathrm{g} / \mathrm{kg}$ bw/day PAs representing the lowest and the highest daily intake previously estimated to originate from use of herbal teas (BfR, 2013). Each parameter was analysed individually, keeping the other parameters to their initial values.

Because of the lack of available in vivo kinetic data for lasiocarpine, to evaluate the performance of the PBK model developed in the present study, the predicted riddelliine blood concentrations obtained with the rat and mouse models were compared with blood concentrations from in vivo kinetic studies in rat and mouse reported in the literature (Williams et al., 2002).

\section{Translation of the in vitro concentration-response curves to in vivo dose-response curves}

Based on the reverse dosimetry approach, the in vitro effect concentrations of lasiocarpine and riddelliine inducing cytotoxicity were considered as surrogate in vivo (free) concentrations in blood that could result in adverse effect on the liver. In the present study, each concentration used in the cytotoxicity assay, corrected by $f_{u b}$ (determined as described above), was set equal to the maximum concentration $\left(\mathrm{C}_{\max }\right)$ of lasiocarpine or riddelliine in the liver blood and the developed PBK models were used to determine the oral dose required that results in this concentration.

\section{BMD analysis of in vitro concentration-response data and of predicted in vivo dose- response data}

The continue model in PROAST programme (version 38.9, The Dutch National Institute for Public Health and the Environment, The Netherlands) was used to analyse the predicted in vivo doseresponse data and to obtain the $\mathrm{BMDL}_{5}-\mathrm{BMDU}_{5}$ (lower/upper limit of the $90 \%$ confidence interval of the benchmark dose that gives a $5 \%$ response) values for liver toxicity. The aim of the use of continue model was to determine $\mathrm{BMDU}_{5}$ value. The goodness of fit application of the models was used to judge if the model can be accepted, using the $\mathrm{P}$ value with $\mathrm{P}>0.05$. Only the models which met the requirements for acceptance of the model fit were considered for the determination of $\mathrm{BMDL}_{5}-$ $\mathrm{BMDU}_{5}$ values. The same analysis was performed to predict in vivo dose-response curves derived upon extrapolation of the concentration-response curves for in vitro cytotoxicity derived from the 
literature (Hayes et al., 1984).

\section{Evaluation of the combined in vitro-PBK modelling approach}

To support the validity and use of the in vitro-in silico approach to obtain a PoD for risk assessment, the predicted $\mathrm{BMDL}_{5}-\mathrm{BMDU}_{5}$ values of lasiocarpine for rat were compared with PoDs derived from in vivo rat acute liver toxicity data from the literature (Dalefield et al., 2016; Jago, 1970; Nolan et al., 1966). For comparison of the predicted in vivo toxicity data to the available in vivo toxicity data form the literature it was assumed that the $\mathrm{BMDL}_{5}-\mathrm{BMDU}_{5}$ from such work, could be a reasonable approximation of an in vivo NOAEL-LOAEL (No/Lowest observed adverse effect level) value.

\section{Results}

\section{In vitro cytotoxicity}

Figure 3 shows the concentration-response curves for the cytotoxicity of lasiocarpine or riddelliine in incubations with rat hepatocytes. The IC50 of lasiocarpine and riddelliine derived from these curves amounted to $10.9 \mu \mathrm{M}$ and $6.3 \mu \mathrm{M}$, respectively as shown in Table 2. The in vitro concentrationresponse curve of lasiocarpine in the present study was comparable with in vitro concentrationresponse curves previously reported by Hayes et al. (Hayes et al., 1984) (Figure 3). Table 2 shows the IC50 values obtained from the concentration-response curves in Figure 3.

- Present study, Lasiocarpine, Rat hepatocytes

- Present study, Riddelliine, Rat hepatocytes

- Hayes et al., 1984, Lasiocarpine, Rat hepatocytes

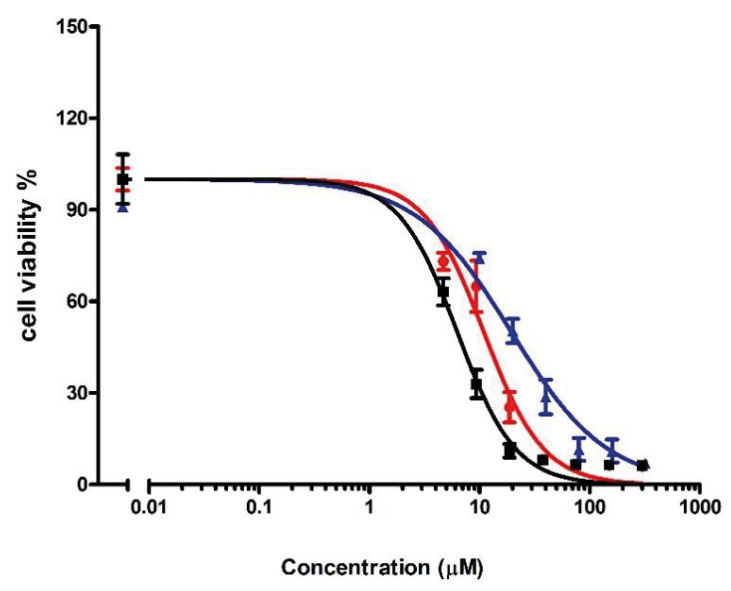

Fig. 3 Concentration-response curves for primary rat hepatocytes treated with increasing concentrations of lasiocarpine (red circles) and riddelliine (black squares) for 24 hours (mean \pm SD) and as reported by Hayes et al. (1984) for lasiocarpine. 
Table 2 IC50 values derived from the concentration-response curves presented in Figure 3.

\begin{tabular}{lll}
\hline Compound & IC50 \pm SD $(\mu \mathrm{M})$ & Studies \\
\hline Lasiocarpine & $10.9 \pm 1.6$ & Present study \\
& $19.5 \pm 4.6$ & (Hayes et al., 1984) \\
\hline Riddelliine & $6.3 \pm 0.9$ & Present study \\
\hline
\end{tabular}

\section{In vitro kinetic data}

Figure $4 \mathrm{a}$ and $\mathrm{b}$ shows the rate of depletion of the PAs in incubations with liver and intestinal microsomes with increasing concentrations of lasiocarpine or riddelliine. The results reveal that lasiocarpine and riddelliine are converted in a concentration-dependent manner. For each reaction, metabolism followed Michaelis-Menten kinetics. The apparent $\mathrm{V}_{\max }$ and $\mathrm{K}_{\mathrm{m}}$ values and the (scaled) catalytic efficiency $\left(\mathrm{V}_{\max } / \mathrm{K}_{\mathrm{m}}\right)$ for the depletion of lasiocarpine and riddelliine derived from these curves are presented in Table 3. The scaled in vivo catalytic efficiency (calculated as $\mathrm{V}_{\max } / \mathrm{K}_{\mathrm{m}}$ ) of liver for conversion of lasiocarpine and riddelliine by rat was 6.3 times and 107.8 times higher than the catalytic efficiency for conversion by intestine, respectively. The total scaled in vivo catalytic efficiency (sum of liver and intestine) for depletion of lasiocarpine was 11 times higher than that for riddelliine. The results reveal that in rat, lasiocarpine and riddelliine are especially converted in the liver, and that intestinal conversion contributes only to a minor extent.

The rate of depletion of riddelliine in incubations with mouse tissue samples and increasing concentrations of riddelliine is shown in Figure $4 c$. The (scaled) catalytic efficiencies $\left(V_{\max } / K_{m}\right)$ for the depletion of riddelliine in mouse tissue are also shown in Table 3. For mouse tissues, the scaled catalytic efficiency of the liver for conversion of riddelliine was 9.6 times higher than the intestine. The total catalytic efficiency for metabolic conversion of riddelliine by rat and mouse liver and intestine was comparable, being only 1.8 times higher in rat than in mouse. Incubations with lung and kidney rat and mouse tissue samples did not result in detectable conversion (data not shown). 
a)

Rat

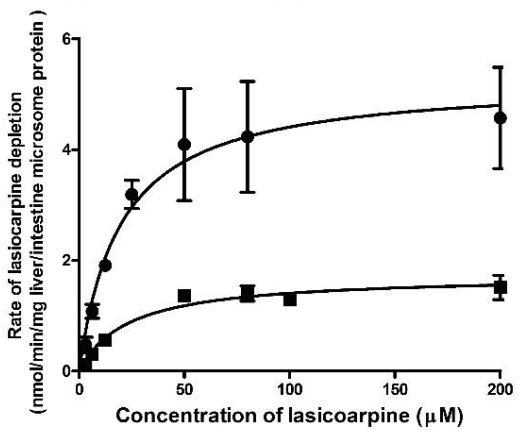

b)
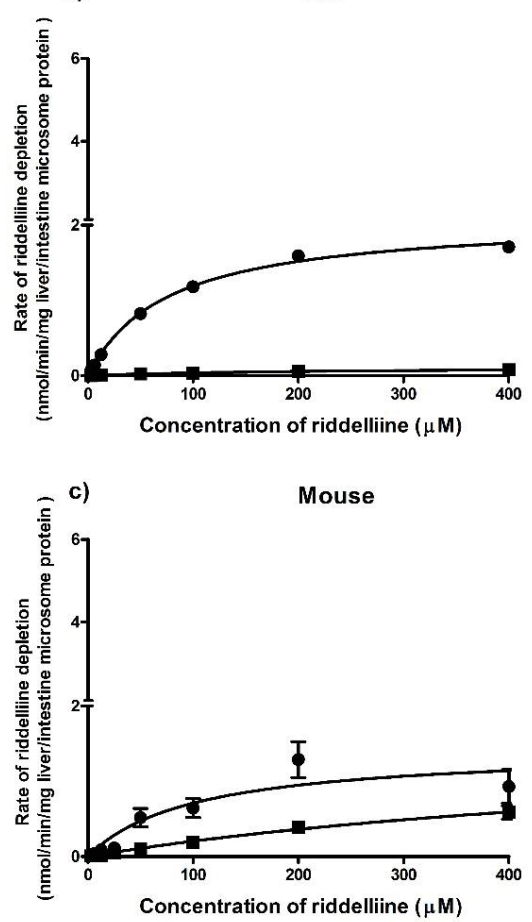

Fig. 4 Concentration-dependent rate of lasiocarpine (a) and riddelliine (b and c) depletion in incubations with rat liver (filled circle) and intestinal (filled square) microsomes (a and b) and mouse liver (filled circle) and intestinal (filled square) microsomes (c). Results represent data of three independent experiments. 
Table 3 Kinetic parameters for lasiocarpine and riddelliine depletion by pooled rat tissue fractions, and for riddelliine depletion by pooled mouse tissue fractions.

\begin{tabular}{|c|c|c|c|c|c|}
\hline $\begin{array}{l}\text { Species } \\
\text { Organ }\end{array}$ & $\begin{array}{l}\mathrm{V}_{\max } \\
(\mathrm{nmol} / \mathrm{min} / \mathrm{mg} \\
\text { microsomal } \\
\text { protein) }\end{array}$ & $\mathrm{K}_{\mathrm{m}}(\mu \mathrm{M})$ & $\begin{array}{l}\text { Catalytic } \\
\text { efficiency } \\
\text { (mL/min/mg } \\
\text { microsomal } \\
\text { protein) }\end{array}$ & $\begin{array}{l}\text { Scaled } V_{\max }{ }^{a} \\
(\mathrm{nmol} / \mathrm{min} / \mathrm{g} \\
\text { tissue) }\end{array}$ & $\begin{array}{l}\text { Scaled catalytic } \\
\text { efficiencyb } \\
\text { (mL/min } / \mathrm{g} \\
\text { tissue) }\end{array}$ \\
\hline
\end{tabular}

\section{Rat}

\section{Lasiocarpine}

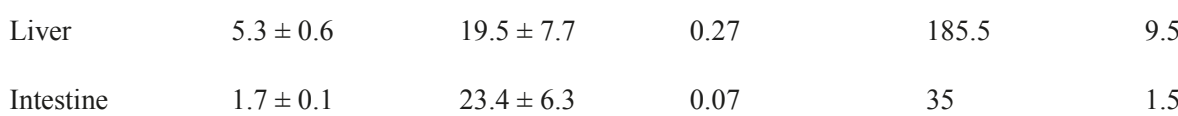

\section{Riddelliine}

\begin{tabular}{|c|c|c|c|c|}
\hline Liver & $2.1 \pm 0.07$ & $75.7 \pm 7.4$ & 0.03 & 73.5 \\
\hline Intestine & $0.1 \pm 0.02$ & $221.0 \pm 71.2$ & 0.0005 & 2.06 \\
\hline
\end{tabular}

Mouse

Riddelliine

$\begin{array}{llllll}\text { Liver } & 1.4 \pm 0.3 & 101.3 \pm 49.9 & 0.01 & 49 & 0.48\end{array}$

$\begin{array}{llllll}\text { Intestine } & 1.6 \pm 0.5 & 695.8 \pm 342.7 & 0.002 & 33 & 0.05\end{array}$

${ }^{a}$ Scaled $V_{\max }$ calculated from the in vitro $\mathrm{V}_{\max }$ based on a microsome protein yield of $35 \mathrm{mg} /(\mathrm{g}$ liver) or 20.6 $\mathrm{mg} /(\mathrm{g}$ small intestine)

${ }^{\mathrm{b}}$ Catalytic efficiency expressed as $\mathrm{mL} / \mathrm{min} /\left(\mathrm{g}\right.$ liver) or $\mathrm{mL} / \mathrm{min} /\left(\mathrm{g}\right.$ intestine) is the ratio of scaled $\mathrm{V}_{\max }$ and $\mathrm{K}_{\mathrm{m}}$

\section{PBK model evaluation}

Due to unavailability of in vivo kinetic data for lasiocarpine, to evaluate the performance of the PBK models, the blood concentration-time curves of riddelliine as predicted by the developed PBK models were compared with the in vivo kinetic data from the literature on the blood concentration of riddelliine in rat and mouse exposed to riddelliine. Williams et al. reported riddelliine blood concentrations in rat and mouse that were orally exposed to $10 \mathrm{mg} / \mathrm{kg}$ bw of riddelliine, and from which blood samples were collected from 0.5-24 hours (Williams et al., 2002). Figure 5a shows that for the mouse model there is only 2 to 6.5 (average $4.6 \pm 2.2$ ) fold difference at time points of $0.5,2,3$, 4, 8, 24 hours between the predicted blood concentrations using the developed PBK model and the observed blood concentration of riddelliine upon a dose of $10 \mathrm{mg} / \mathrm{kg}$ bw in mouse (Williams et al., 2002). When comparing the predicted blood concentrations with in vivo blood concentrations of riddelliine upon a dose of $10 \mathrm{mg} / \mathrm{kg}$ bw in rat, the results showed that the predicted values are 0.9 to 
23.7 (average $14.5 \pm 9.1$ ) fold higher at time points of $0.5,1,2,3,4,8,24$ hours than the observed blood concentrations. Further, the differences between the predicted and observed $\mathrm{C}_{\max }$ of riddelliine at a dose of $10 \mathrm{mg} / \mathrm{kg}$ bw, were 2-fold for mouse and 9.7-fold for rat (Figure 5). The predicted blood concentration-time curves of riddelliine in mouse using the two individual Ka values are shown in the Supplementary data (Figure S1). As shown in Figure S1, the two Ka values for riddelliine result in different predicted blood concentration-time curves and in $\mathrm{C}_{\max }$ values that are 3.2- fold different.

In addition, the predicted AUC values were also calculated. The observed AUC were $516 \mathrm{ng} \mathrm{h} / \mathrm{mL}$ for rat and $1307 \mathrm{ng} \mathrm{h} / \mathrm{mL}$ for mouse (Williams et al., 2002), while the predicted AUC values were 5323 $\mathrm{ng} \mathrm{h} / \mathrm{mL}$ for rat and $6113 \mathrm{ng} \mathrm{h} / \mathrm{mL}$ for mouse, respectively. Thus, the predicted AUC for rat was 10.3fold higher than the observed AUC, while the predicted AUC for mouse was 4.7-fold higher than the observed AUC.
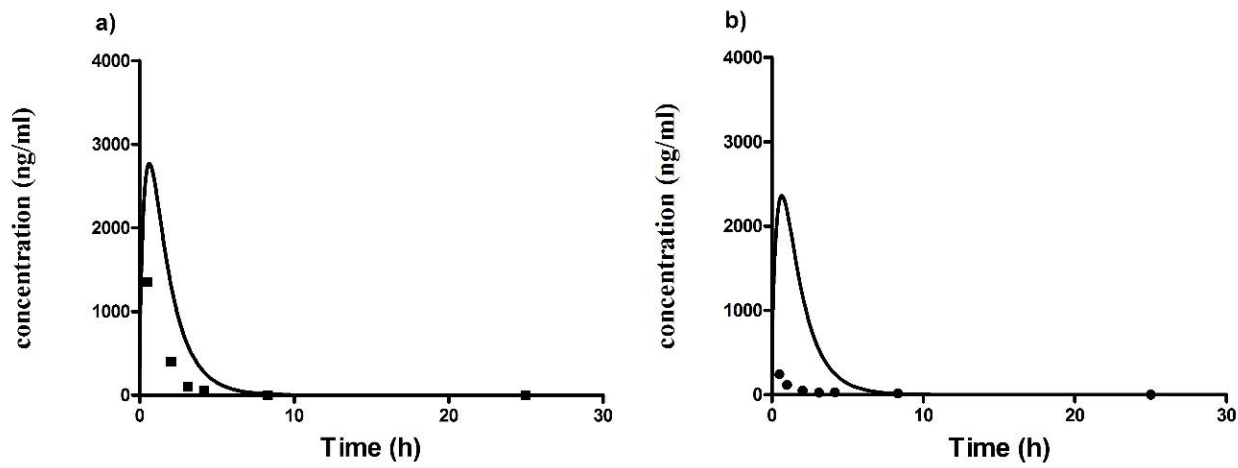

Fig. 5 PBK modelling-predicted time-dependent blood concentrations (black line) and reported time-dependent blood concentrations (filled square) for riddelliine in mouse (a) or (filled circle) in rat (b) upon a dose of 10 $\mathrm{mg} / \mathrm{kg}$ bw.

\section{Sensitivity analysis}

To further evaluate the performance of the developed models, a sensitivity analysis was performed to assess the parameters that affect the prediction of the $\mathrm{C}_{\max }$ of lasiocarpine and riddelliine in liver blood. The sensitivity analysis was performed at an oral dose of 0.008 and $0.144 \mu \mathrm{g} / \mathrm{kg}$ bw/day. These PA dose levels represent the reported lowest and the highest daily intake originating from use of herbal teas (BfR, 2013). Only the parameters that result in a normalized sensitivity coefficient higher than 0.1 (in absolute value) are shown in Figure 6. The results obtained reveal that the fraction of liver volume, partition coefficient of liver, the uptake rate constant, the liver microsomal protein yield, the kinetic parameters $\left(\mathrm{V}_{\max }\right.$ and $\left.\mathrm{K}_{\mathrm{m}}\right)$ for depletion of lasiocarpine and riddelliine in the liver were the most sensitive parameters in the rat PBK models for lasiocarpine and riddelliine. Compared to the rat PBK 
for riddelliine, the parameters related to the intestine are more influential in the rat PBK model for lasiocarpine (Figure 6A). Comparison of the sensitivity analysis performed at 0.008 and $0.144 \mu \mathrm{g} / \mathrm{kg}$ bw/day reveals that the influence of all parameters was not dose-dependent in the rat PBK model for lasiocarpine and riddelliine (Figure 6).
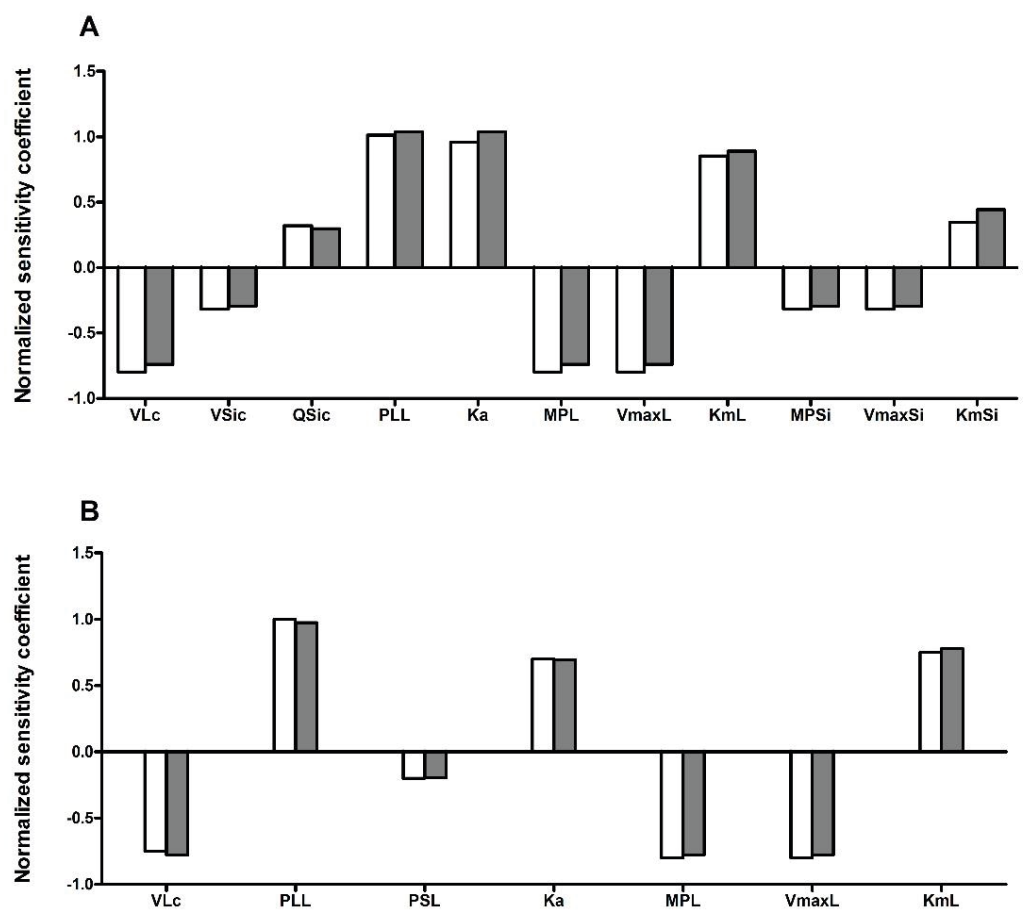

Fig. 6 Normalized sensitivity coefficients for the parameters of the rat PBK model for lasiocarpine (A) and riddelliine (B) on predicted $\mathrm{C}_{\max }$ in liver blood values at a single oral dose of $0.008 \mu \mathrm{g} / \mathrm{kg}$ bw/day (white bars) and $0.144 \mu \mathrm{g} / \mathrm{kg}$ bw/day (grey bars) PAs. VLc $=$ fraction of liver volume, $\mathrm{VSi}=$ fraction of small intestine volume, $\mathrm{QSi}=$ blood flow to small intestine, $\mathrm{PLL}=$ liver/blood partition coefficient, $\mathrm{PSL}=$ slowly perfused tissue/blood partition coefficient, $\mathrm{Ka}=$ uptake rate constant, MPL and MPSi $=$ liver and small intestinal microsomal protein yield, $\mathrm{V}_{\max } \mathrm{L}$ and $\mathrm{K}_{\mathrm{m}} \mathrm{L}=$ the maximum rate of depletion and the Michaelis-Menten constant for depletion of lasiocarpine or riddelliine in liver, $\mathrm{V}_{\max } \mathrm{Si}$ and $\mathrm{K}_{\mathrm{m}} \mathrm{Si}$ = the maximum rate of depletion and the Michaelis-Menten constant for depletion of lasiocarpine or riddelliine in small intestine.

Translation of the in vitro concentration-response curves to in vivo dose-response curves, and application of PROAST modelling on predicted dose-response data to derive PoDs

Figure 7 shows the predicted in vivo dose-response curves for liver toxicity of lasiocarpine and riddelliine in rat using PBK modelling-facilitated reverse dosimetry. The two predicted dose-response curves were derived from the in vitro cytotoxicity concentration-response curves obtained with rat hepatocytes (Figure 3) based on the $C_{\max }$ assuming the in vitro concentration to be equal to the $C_{\max }$ 
of unbound PA in liver blood. The $\mathrm{fub}_{\mathrm{ub}}$ of lasiocarpine and riddelliine in rat serum was estimated to be 0.64 and 0.66 , respectively. Table 4 displays the range of $\mathrm{BMDL}_{5}-\mathrm{BMDU}_{5}$ values derived from these predicted dose-response curves. The predicted $\mathrm{BMDL}_{5}-\mathrm{BMDU}_{5}$ values for lasiocarpine and riddelliine in rats were 23-34.4 and 4.9-8.4 mg/kg bw/day, respectively. When converting the concentrationresponse curves from Hayes et al. (1984) for rat hepatocytes depicted in Figure 3 and using the same PBK model, the predicted $\mathrm{BMDL}_{5}-\mathrm{BMDU}_{5}$ values for lasiocarpine were $34.5-72.1 \mathrm{mg} / \mathrm{kg} \mathrm{bw} / \mathrm{day}$ (Table 4). When using the individual Ka for prediction of the $\mathrm{BMDL}_{5}-\mathrm{BMDU}_{5}$ values, the predicted $\mathrm{BMDL}_{5}-\mathrm{BMDU}_{5}$ values for riddelliine in rat result in the ranges of $3.3-14.6$ and $1.0-2.2 \mathrm{mg} / \mathrm{kg} \mathrm{bw} /$ day, respectively.

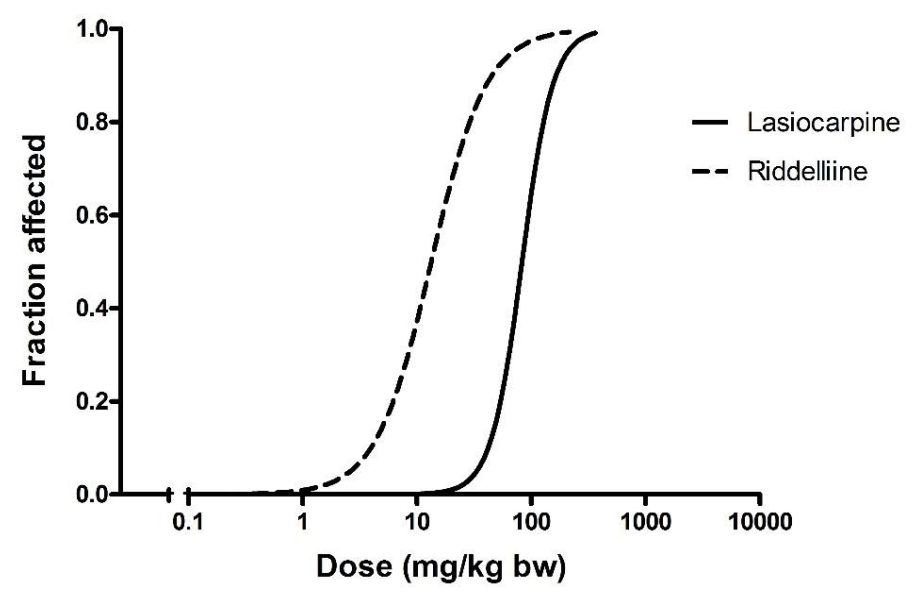

Fig. 7 Predicted in vivo dose-response curves for acute liver toxicity of lasiocarpine and riddelliine in rat. The solid line represents the predicted in vivo dose-response curves for lasiocarpine and the dashed line represents the predicted dose-response curve for riddelliine.

Table 4 Predicted $\mathrm{BMDL}_{5}-\mathrm{BMDU}_{5}$ values derived from the dose-response curves predicted using PBK modelling-facilitated reverse dosimetry to convert the in vitro concentration-response curves as obtained in the present study and obtained from the literature (Figure 3) to in vivo dose-response curves.

\begin{tabular}{llll}
\hline Compound & Cell model & $\begin{array}{l}\text { Predicted BMDL5-BMDU } 5 \\
(\mathrm{mg} / \mathrm{kg} \text { bw/day })\end{array}$ & $\begin{array}{l}\text { Source in vitro } \\
\text { concentration-response } \\
\text { curve }\end{array}$ \\
\hline $\begin{array}{l}\text { Lasiocarpine } \\
\text { Riddelliine }\end{array}$ & Rat hepatocytes & $23.0-34.4$ & Present study \\
Rasiocarpine & Rat hepatocytes & $4.9-8.4$ & Present study \\
& & $34.5-72.1$ & (Hayes et al., 1984) \\
\hline
\end{tabular}




\section{Evaluation of the predicted PoDS for in vivo liver toxicity}

Due to the absence of in vivo acute liver toxicity data reported in the literature for riddelliine, the predicted PoDs for in vivo liver toxicity could only be evaluated for lasiocarpine. To this end, the $\mathrm{BMDL}_{5}-\mathrm{BMDU}_{5}$ values presented in Table 4 were compared with in vivo reported data for acute liver toxicity of lasiocarpine as available in the literature (Dalefield et al., 2016; Jago, 1970; Nolan et al., 1966). Table 5 presents an overview of literature data on acute liver toxicity of lasiocarpine in rat, measuring endpoints including megalocytosis, the mitotic index value, or the alanine amino transferase concentration in serum as a measure of acute liver toxicity. The data from these studies were not suitable for BMD analysis due to the limited number of data points and insufficient distribution of the data points over the dose-response curves. Therefore, the NOAEL values and/or the range of estimated NOAEL to LOAEL values that could be derived from these studies were taken as the PoDs (Table 5). When only the LOAEL was available, the NOAEL was calculated using the LOAEL divided by a factor of 10 (Barnes et al., 1988).

Figure 8 shows a comparison of the predicted range of $\mathrm{BMDL}_{5}-\mathrm{BMDU}_{5}$ values for in vivo liver toxicity of lasiocarpine and the PoDs derived from in vivo literature rat data for liver toxicity of lasiocarpine. The literature values for single oral exposure are the ones that can best be compared to the values predicted in the present study. Values for IP or oral repeated exposure appear to be somewhat lower probably because of these different dose regimes. The predicted $\mathrm{BMDL}_{5}-\mathrm{BMDU}_{5}$ values derived from the present study appear to fall well within the range of PoDs obtained from the available oral single dose in vivo studies. The $\mathrm{BMDL}_{5}$, a possible $\mathrm{PoD}$ for evaluation of acute toxicity of PAs, determined from predicted concentration-response curves was 1.9- to 2.9-fold higher than the NOAELs derived as corresponding PoD from the experimental data. The $\mathrm{BMDU}_{5}$ predicted in the present study was 2.3- to 3.5-fold lower compared to the LOAELs derived as corresponding PoD from the in vivo studies. The results indicate that the data and range of $\mathrm{PoD}$ values derived using our combined in vitro-PBK modelling approach are a good approximation to the in vivo acute liver toxicity of lasiocarpine. 


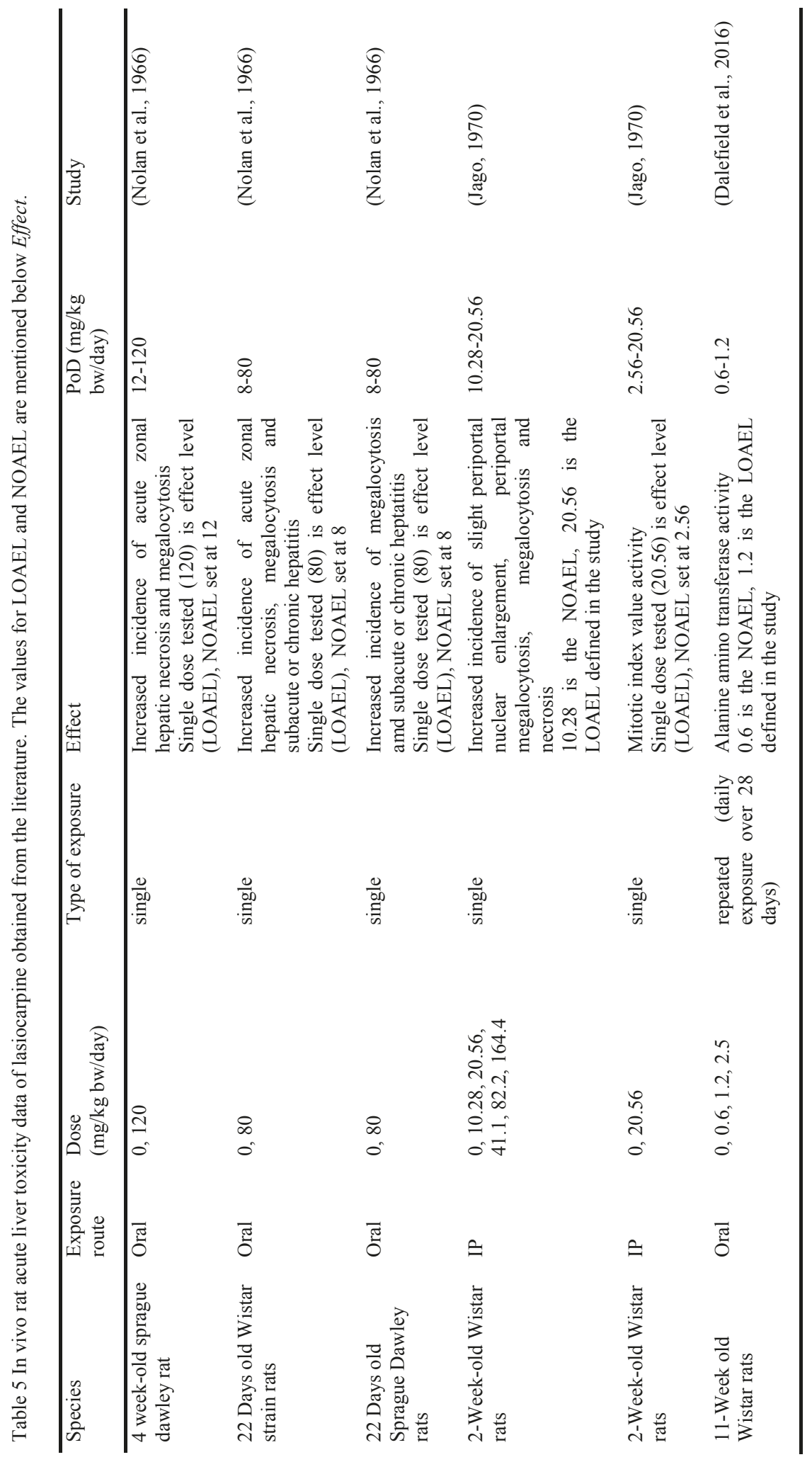




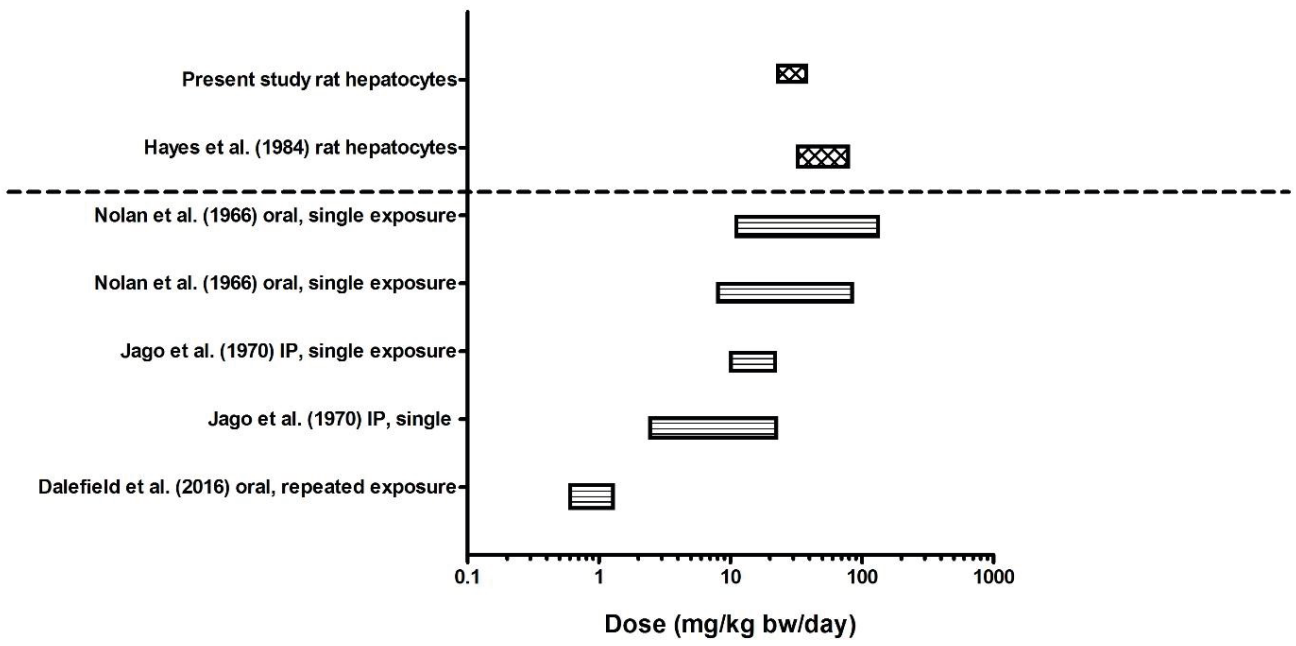

Fig.8 BMDL 5 -BMDU 5 values for acute liver toxicity of lasiocarpine in rat predicted by the PBK modellingfacilitated reverse dosimetry approach using data for rat hepatocytes from the present study and literature data for rat hepatocytes (rectangle bars filled with grid) as compared to comparable PoDs (rectangle bars filled with horizontal lines) derived from in vivo liver toxicity studies as shown in Tabls 5 upon oral or IP exposure. Range of estimated PoD from in vivo data = estimated NOAEL to LOAEL.

\section{Discussion}

The aim of present study was to assess whether a physiologically based kinetic (PBK) modellingfacilitated reverse dosimetry approach could adequately predict in vivo dose-response curves for hepatotoxicity of PAs in rats. Especially 1,2-unsaturated PAs are of concern because of their hepatotoxicity and carcinogenicity. However, of the large group of 1,2-unsaturated PAs in vivo data for liver toxicity are only available for lasiocarpine (Dalefield et al., 2016; Jago, 1970; Nolan et al., 1966). A method to estimate liver toxicity for related PAs using read-across from lasiocarpine based on quantitative insight into the relative level of their bioactivation and detoxification would enable priority setting in the further evaluation of the toxicity of PAs for which no toxicity data are availabe. To facilitate this approach the aim of the present study was to develop a PBK model for PAs and use this model to generate a proof of principle that liver toxicty of the model PA lasiocarpine can be adequately predicted by this comined in silico-in vitro alternative tetsing strategy.

In the present study, the in vitro concentration-response curves of lasiocarpine and riddelliine in primary rat hepatocytes were used as the in vitro toxicity data. Riddelliine and lasiocarpine were selected as the model PAs, because for riddelliine data on dose- and time-dependent blood levels in mouse and rats were available enabling evaluation of the PBK models, while for lasiocarpine in vivo data for liver toxicity were available, enabling valuation of the final prediction of the in vivo liver 
toxicity. Primary rat liver hepatocytes were selected as the in vitro model because they are expected to best reflect the intrinsic sensitivity and metabolism of liver cells in vivo. Furthermore, hepatocytes were used before to characterise in vitro liver toxicity of lasiocarpine (Hayes et al., 1984; Moore et al., 1989). The results for the in vitro cytotoxicity of lasiocarpine in rat primary hepatocytes obtained in the present study were comparable to the literature data (Hayes et al., 1984).

The PBK models developed could be evaluated based on in vivo kinetic data available for riddelliine (Williams et al., 2002). The predicted riddelliine blood concentrations by the PBK model for mouse were 2 to 6.5 (average $4.6 \pm 2.2$ ) fold different from those reported by Williams et al. (2002) and for the rat PBK model the predicted blood concentrations were 0.9 to 23.7 (average $14.5 \pm 9.1$ ) fold different from those reported by Williams et al. (2002). In a subsequent step, the PBK models were used to convert the in vitro cytotoxicity data for lasiocarpine and riddelliine to in vivo dose-response curves for liver toxicity, enabling to assess the validity and use of the PBK model for reverse dosimetry, by comparison of the predicted toxicity data to available in vivo toxicity data for lasiocarpine. The predicted $\mathrm{BMDL}_{5}-\mathrm{BMDU}_{5}$ values derived from the present study appeared to fall well within the range of PoDs obtained from the available oral single dose in vivo studies. The results indicate that the data and range of PoD values derived using our combined in vitro-in silico PBK modelling approach are a good approximation of the in vivo acute liver toxicity of lasiocarpine.

In addition, the $\mathrm{BMDL}_{5}-\mathrm{BMDU}_{5}$ values predicted for riddelliine were 2.7 to 7.0 times lower than those values predicted for lasiocarpine. This indicates that in vivo riddelliine is predcited to induce liver toxicity at lower dose levels than lasiocarpine. In line with this, the in vitro cytotoxicity of riddelliine was also higher than that of lasiocarpine. The higher in vivo toxicity of ridelliine may in part be due to the fact that there are differences in kinetics which revealed a slower clearance of riddelliine compared to lasiocarpine. Given all the results above, it is concluded that the developed PBK modeling-facilitated reverse dosimetry approach provides a promising method to predict acute liver toxicity in vivo for lasiocarpine, and related PAs. Based on the results of the sensitivity analysis (Figure 6), the model preditions are highly influenced by the kinetic constant Ka for uptake of the PAs in the small intestine. In current study, the predicted $\mathrm{BMDL}_{5}-\mathrm{BMDU}_{5}$ values derived when using the average Ka value fall within the ranges of predicted $\mathrm{BMDL}_{5}-\mathrm{BMDU}_{5}$ values using the individual Ka values. Nevertheless the method used to determine the $P_{\text {app }}$ value and resulting Ka for intestinal uptake of PAs may need further refinement.

In addition it is also of interest to compare the PoDs predicted for acute oral toxicity in rats, to what can be derived from reported cases on actue toxicity in humans. This includes for example cases in Afghanistan in 1976 (Mohabbat et al., 1976), in Jamaica in 1954 (Bras et al., 1954), and in India in 1975 (Tandon et al., 1976). In fact, these reports reveal that during the past century, a lot of acute PAs poisoning cases took place in central Asia, while other cases have been reported for other countries 
with poorly developed agricultural systems (Dharmananda, 2001). Culvenor estimated the dose and duration of exposure to the toxic PAs that may lead to liver toxicity in humans (Culvenor, 1983) and concluded that an intake of $0.7-1.5 \mathrm{mg} / \mathrm{kg}$ bw/day for riddelliine for 2 weeks can induce hepatotoxicity. The predicted $\mathrm{BMDL}_{5}-\mathrm{BMDU}_{5}$ value for acute liver toxicity of riddelliine in rats reported in the present study amounted to $4.9-8.4 \mathrm{mg} / \mathrm{kg}$ bw/day. Comparison of these values suggests that humans might be more sensitive than rats, although it should be kept in mind that the human study was based on more than a single exposure and that estimation of dose levels in human case studies is generally not acurate. In a next step, the PBK modelling based approach developed in the present study will be modified to predict human liver toxicity using human hepatocytes and the PBK model modified to describe human kinetics.

In conclusion, we showed that PBK modelling-facilitated reverse dosimetry can translate in vitro concentration-response curves to in vivo dose-response curves for acute liver toxicity of lasiocarpine and riddelliine. $\mathrm{BMDL}_{5}-\mathrm{BMDU}_{5}$ values could be derived that were in line with available in vivo data. This study provides another proof-of-principle for integrating in vitro toxicity data and PBK modelling-facilitated reverse dosimetry to obtain a promising alternative testing strategy in risk and safety evaluation of chemicals.

\section{Acknowledgements}

This work was funded by a grant from the China Scholarship Council to Lu Chen (grant No. 201508650023).

The riddelliine was kindly provided by Patrick P.J. Mulder from RIKILT Wageningen University \& Research, Wageningen, The Netherlands. 


\section{References}

Abdullah, R., Alhusainy, W., Woutersen, J., Rietjens, I.M., Punt, A., 2016. Predicting points of departure for risk assessment based on in vitro cytotoxicity data and physiologically based kinetic (PBK) modeling: The case of kidney toxicity induced by aristolochic acid I. Food Chemical Toxicol. 92, 104-116.

Barnes, D.G., Dourson, M., Dourson, M., Preuss, P., Barnes, D.G., Bellin, J., Derosa, C., Engler, R., Erdreich, L., Farber, T., Fenner-Crisp, P., Francis, E., Ghali, G., Hill, R., Irene, S., Marcus, W., Patrick, D., Perlin, S., Revesz, A., Rubenstein, R., Stara, J., Wiltse, J., Zaragosa, L., 1988. Reference dose (RfD): Description and use in health risk assessments. Regul Toxicol Pharmacol. 8, 471-486.

BfR, 2013. pyrrolizidine alkaloids in herbal teas and teas. BfR, 1-29.

Bovee, T.F., Helsdingen, R.J., Hoogenboom, R.L., de Nijs, M.W., Liu, X., Vrieling, K., Klinkhamer, P.G., Peijnenburg, A.A., Mulder, P.P., 2015. Are effects of common ragwort in the Ames test caused by pyrrolizidine alkaloids? Mutation Research/Fundamental and Molecular Mechanisms of Mutagenesis. 778, 1-10.

Bras, G., Jelliffe, D.B., Stuart, K.L., 1954. Veno-occlusive disease of liver with nonportal type of cirrhosis, occurring in Jamaica. Arch Pathol. 57, 285-300.

Brown, R.P., Delp, M.D., Lindstedt, S.L., Rhomberg, L.R., Beliles, R.P., 1997. Physiological Parameter Values for Physiologically Based Pharmacokinetic Models. Toxicol Ind Health. 13, 407-484.

Bull, L.B., Culvenor, C.t., Dick, A., 1968. The pyrrolizidine alkaloids: their chemistry, pathogenicity and other biological properties. The Quarterly Review of Biology 45, no. 1 (Mar., 1970): 67.

Cubitt HE, Houston JB, Galetin A., 2009. Relative importance of intestinal and hepatic glucuronidation-impact on the prediction of drug clearance. Pharmaceutical research. May 1;26(5):1073.

Chan, P., 1993. NTP technical report on the toxicity studies of Riddelliine (CAS No. 23246-96-0) Administered by Gavage to F344 Rats and B6C3F1 Mice. Toxicity report series 27, 1-D9.

Chan, P.C., Haseman, J.K., Prejean, J., Nyska, A., 2003. Toxicity and carcinogenicity of riddelliine in rats and mice. Toxicol Lett. 144, 295-311.

Chou, M.W., Jian, Y., Williams, L.D., Xia, Q., Churchwell, M., Doerge, D.R., Fu, P.P., 2003. Identification of DNA adducts derived from riddelliine, a carcinogenic pyrrolizidine alkaloid. Chem Res Toxicol. 16, 1130-1137.

Culvenor, C., 1983. Estimated intakes of pyrrolizidine alkaloids by humans. A comparison with dose rates causing tumors in rats. J Toxicol Environ Health, Part A Current Issues 11, 625-635.

Dalefield, R.R., Gosse, M.A., Mueller, U., 2016. A 28-day oral toxicity study of echimidine and lasiocarpine in Wistar rats. Regul Toxicol Pharmacol. 81, 146-154.

DeJongh, J., Verhaar, H.J., Hermens, J.L., 1997. A quantitative property-property relationship (QPPR) approach to estimate in vitro tissue-blood partition coefficients of organic chemicals in rats and humans. Arch Toxicol. 72, 17-25.

Dharmananda, S., 2001. Safety issues affecting herbs: pyrrolizidine alkaloids.

EFSA, 2011. Panel on Contaminants in the Food Chain (CONTAM); Scientific Opinion on Pyrrolizidine alkaloids. EFSA Journal. 9:2406:2134 pp.

EFSA, 2013. Scientific opinion on pyrrolizidine alkaloids in food and feed. EFSA Journal. 9, 1-134.

Evans, M.V., Andersen, M.E., 2000. Sensitivity analysis of a physiological model for 2, 3, 7, 8 tetrachlorodibenzo-p-dioxin (TCDD): assessing the impact of specific model parameters on sequestration in liver and fat in the rat. Toxicol Sci. 54, 71-80.

Fu, P.P., 2016. Pyrrolizidine Alkaloids: Metabolic activation pathways leading to liver tumor initiation. Chem Res Toxicol. 30, 81-93

Fu, P.P., Xia, Q., Lin, G., Chou, M.W., 2002. Genotoxic pyrrolizidine alkaloids-mechanisms leading to DNA adduct formation and tumorigenicity. Int J Mol Sci. 3, 948-964.

Fu, P.P., Xia, Q., Lin, G., Chou, M.W., 2004. Pyrrolizidine alkaloids-genotoxicity, metabolism enzymes, metabolic activation, and mechanisms. Drug Metab Rev. 36, 1-55. 
Hayes, M.A., Roberts, E., Jago, M.V., Safe, S.H., Farber, E., Cameron, R.C., 1984. Influences of various xenobiotic inducers on cytocidal toxicity of lasiocarpine and senecionine in primary cultures of rat hepatocytes. J Toxicol Environ Health. 14, 683-694.

Hirono, I., Haga, M., Fujii, M., Matsuura, S., Matsubara, N., Nakayama, M., Furuya, T., Hikichi, M., Takanashi, H., Uchida, E., 1979. Induction of hepatic tumors in rats by senkirkine and symphytine. J Natl Cancer Inst. 63, 469-472.

Hirono, I., Mori, H., Culvenor, C.C., 1976. Carcinogenic activity of coltsfoot, Tussilago farfara L. Jpn. J. Cancer Res. 67, 125-129.

Hirono, I., Mori, H., Yamada, K., Hirata, Y., Haga, M., Tatematsu, H., Kanie, S., 1977. Carcinogenic activity of petasitenine, a new pyrrolizidine alkaloid isolated from Petasites japonicus Maxim. $\mathrm{J}$ Natl Cancer Inst. 58, 1155-1157.

Hirono., I., Haga., M., Fujii., M., Matsuura., S., Matsubara., N., Nakayama., M., Furuya., T., Hikichi., M., Takanashi., H., Uchida., E., Hosaka., S., Ueno, I., 1979. Induction of Hepatic Tumors in Rats by Senkirkine and Symphytine. JNCI 63, 469-472.

Hou, T.J., Zhang, W., Xia, K., Qiao, X.B., Xu, X.J., 2004. ADME evaluation in drug discovery. 5. Correlation of Caco-2 permeation with simple molecular properties. J Chem Inf Comput Sci. 44, 1585-1600.

IARC, 1976. IARC Monographs on Evaluation of Carcinogenic Risk of chemicals to Man: Some Naturally Occuring Substances. 10.

IARC, 2002. Some traditional herbal medicines, some mycotoxins, naphthalene and styrene. IARC monographs on the evaluation of carcinogenic risks to humans. 82 .

Jago, M.V., 1970. A method for the assessment of the chronic hepatoxicity of pyrrolizidine alkaloids. Immunol Cell Biol. 48(1), p.93.

Kuhara, K., Takanashi, H., Hirono, I., Furuya, T., Asada, Y., 1980. Carcinogenic activity of clivorine, a pyrrolizidine alkaloid isolated from Ligularia dentata. Cancer Lett. 10, 117-122.

Kumana, C., Ng, M., Lin, H., Ko, W., Wu, P.-C., Todd, D., 1985. Herbal tea induced hepatic venoocclusive disease: quantification of toxic alkaloid exposure in adults. Gut 26, 101-104.

Li, H., Zhang, M., Vervoort, J., Rietjens, I.M., van Ravenzwaay, B., Louisse, J., 2017. Use of physiologically based kinetic modeling-facilitated reverse dosimetry of in vitro toxicity data for prediction of in vivo developmental toxicity of tebuconazole in rats. Toxicol Lett. 266, 85-93.

Li, N., Xia, Q., Ruan, J., P Fu, P., Lin, G., 2011. Hepatotoxicity and tumorigenicity induced by metabolic activation of pyrrolizidine alkaloids in herbs. Curr Drug Metab. 12, 823-834.

Lin, G., Cui, Y.-Y., Hawes, E.M., 1998. Microsomal formation of a pyrrolic alcohol glutathione conjugate of clivorine. Drug Metab Dispos. 26, 181-184.

Lin, G., Cui, Y.-Y., Hawes, E.M., 2000. Characterization of rat liver microsomal metabolites of clivorine, an hepatotoxic otonecine-type pyrrolizidine alkaloid. Drug Metab Dispos. 28, 14751483.

Lin, G., Wang, J.Y., Li, N., Li, M., Gao, H., Ji, Y., Zhang, F., Wang, H., Zhou, Y., Ye, Y., 2011. Hepatic sinusoidal obstruction syndrome associated with consumption of Gynura segetum. J. Hepatol. 54, 666-673.

Louisse, J., Bosgra, S., Blaauboer, B.J., Rietjens, I.M., Verwei, M., 2015. Prediction of in vivo developmental toxicity of all-trans-retinoic acid based on in vitro toxicity data and in silico physiologically based kinetic modeling. Arch Toxicol. 89, 1135-1148.

Louisse, J., de Jong, E., van de Sandt, J.J., Blaauboer, B.J., Woutersen, R.A., Piersma, A.H., Rietjens, I.M., Verwei, M., 2010. The use of in vitro toxicity data and physiologically based kinetic modeling to predict dose-response curves for in vivo developmental toxicity of glycol ethers in rat and man. Toxicological sciences : an official journal of the Society of Toxicology 118, 470484.

Mattocks, A., 1986. Chemistry and toxicology of pyrrolizidine alkaloids. London: Academic Press.

Medinsky, M.A., Leavens, T.L., Csanády, G.A., Gargas, M.L., Bond, J.A., 1994. In vivo metabolism of butadiene by mice and rats: a comparison of physiological model predictions and experimental data. Carcinogenesis. 15, 1329-1340. 
Miranda, C.L., Reed, R.L., Guengerich, F.P., Buhler, D.R., 1991. Role of cytochrome P450IIIA4 in the metabolism of the pyrrolizidine alkaloid senecionine in human liver. Carcinogenesis 12 , 515-519.

Mohabbat, O., Younos, M.S., Merzad, A., Srivastava, R., Sediq, G.G., Aram, G., 1976. An outbreak of hepatic veno-occlusive disease in north-western Afghanistan. The Lancet 308, 269-271.

Moore, D.J., Batts, K.P., Zalkow, L.L., Fortune Jr, G.T., Powis, G., 1989. Model systems for detecting the hepatic toxicity of pyrrolizidine alkaloids and pyrrolizidine alkaloid N-oxides. Toxicol Appl Pharmacol. 101, 271-284.

Mori, H., Sugie, S., Yoshimi, N., Asada, Y., Furuya, T., Williams, G.M., 1985. Genotoxicity of a variety of pyrrolizidine alkaloids in the hepatocyte primary culture-DNA repair test using rat, mouse, and hamster hepatocytes. Cancer Res. 45, 3125-3129.

Nolan, J.P., Scheig, R.L., Klatskin, G., 1966. Delayed hepatitis and cirrhosis in weanling rats following a single small dose of the senecio alkaloid, lasiocarpine. AM J PATHOL. 49, 129151.

NTP, 1978. Bioassay of lasiocarpine for possible carcinogenicity, National Cancer Institute carcinogenesis technical report series, p. 1.

NTP, 2003. Toxicology and carcinogenesis studies of riddelliine (CAS No. 23246-96-0) in F344/N rats and B6C3F1 mice (gavage studies), National Toxicology Program technical report series, 2003/07/05 ed, pp. 1-280.

Prakash, A.S., Pereira, T.N., Reilly, P.E., Seawright, A.A., 1999. Pyrrolizidine alkaloids in human diet. Mutat Res Genet Toxicol Environ Mutagen. 443, 53-67.

Roeder, E., Pflueger, T., 1995. Analysis of pyrrolizidine alkaloids: A competitive enzyme - linked immunoassay (ELISA) for the quantitative determination of some toxic pyrrolizidine alkaloids. Natural toxins. 3, 305-309.

Roulet, M., Laurini, R., Rivier, L., Calame, A., 1988. Hepatic veno-occlusive disease in newborn infant of a woman drinking herbal tea. J. Pediatr. 112, 433-436.

Schoental, R., 1970. Hepatotoxic activity of retrorsine, senkirkine and hydroxysenkirkine in newborn rats, and the role of epoxides in carcinogenesis by pyrrolizidine alkaloids and aflatoxins. Nature. 227, 401-402.

Schoental, R., Bensted, J., 1963. Effects of whole body irradiation and of partial hepatectomy on the liver lesions induced in rats by a single dose of retrorsine, a pyrrolizidine (Senecio) alkaloid. $\mathrm{Br}$ J Cancer. 17, 242.

Schoental, R., Head, M.A., Peacock, P., 1954. Senecio alkaloids: primary liver tumours in rats as a result of treatment with (1) a mixture of alkaloids from S. jacobaea Lin;(2) retrorsine;(3) isatidine. Br J Cancer. 8, 458.

Shumaker, R., Robertson, K., Hsu, I., Allen, J., 1976. Neoplastic transformation in tissues of rats exposed to monocrotaline or dehydroretronecine. J Natl Cancer Inst. 56, 787-790.

Sperl, W., Stuppner, H., Gassner, I., Judmaier, W., Dietze, O., Vogel, W., 1995. Reversible hepatic veno-occlusive disease in an infant after consumption of pyrrolizidine-containing herbal tea. Eur J Pediatr. 154, 112-116.

Stegelmeier, B., Edgar, J., Colegate, S., Gardner, D., Schoch, T., Coulombe, R., Molyneux, R., 1999. Pyrrolizidine alkaloid plants, metabolism and toxicity. J Nat Toxins. 8, 95-116.

Strikwold, M., Spenkelink, B., de Haan, L.H., Woutersen, R.A., Punt, A., Rietjens, I.M., 2017. Integrating in vitro data and physiologically based kinetic (PBK) modelling to assess the in vivo potential developmental toxicity of a series of phenols. Arch Toxicol. 91, 2119-2133.

Tandon, B.N., Tandon, H., Tandon, R., Narndranathan, M., Joshi, Y., 1976. An epidemic of venoocclusive disease of liver in central India. The Lancet. 308, 271-272.

van Liempd, S., Morrison, D., Sysmans, L., Nelis, P., Mortishire-Smith, R., 2011. Development and validation of a higher-throughput equilibrium dialysis assay for plasma protein binding. JALA: J Lab Autom. 16, 56-67. 
Waters, N.J., Jones, R., Williams, G., Sohal, B., 2008. Validation of a rapid equilibrium dialysis approach for the measurement of plasma protein binding. J Pharm Pharm Sci. 97, 4586-4595.

White, I.N., 1976. The role of liver glutathione in the acute toxicity of retrorsine to rats. Chem Biol Interact. 13, 333-342.

Wiedenfeld, H., 2011. Plants containing pyrrolizidine alkaloids: toxicity and problems. Food Addit Contam: Part A 28, 282-292.

Wiedenfeld, H., 2013. Alkaloids derived from ornithine: pyrrolizidine alkaloids, Natural Products. 359-379.

Wiedenfeld, H., Edgar, J., 2011. Toxicity of pyrrolizidine alkaloids to humans and ruminants. Phytochem Rev. 10, 137-151.

Williams, L., Chou, M.W., Yan, J., Young, J.F., Chan, P.C., Doerge, D.R., 2002. Toxicokinetics of riddelliine, a carcinogenic pyrrolizidine alkaloid, and metabolites in rats and mice. Toxicol Appl Pharmacol. 182, 98-104.

Yan, C.C., Huxtable, R.J., 1995a. The effect of the pyrrolizidine alkaloids, monocrotaline and trichodesmine, on tissue pyrrole binding and glutathione metabolism in the rat. Toxicon. 33, 627-634.

Yan, C.C., Huxtable, R.J., 1995b. Relationship between glutathione concentration and metabolism of the pyrrolizidine alkaloid, monocrotaline, in the isolated, perfused liver. Toxicol Appl Pharmacol. 130, 132-139.

Yan, J., Nichols, J., Yang, Y.-C., Fu, P.P., Chou, M.W., 2002. Detection of riddelliine-derived DNA adducts in blood of rats fed riddelliine. Int J Mol Sci. 3, 1019-1026. 


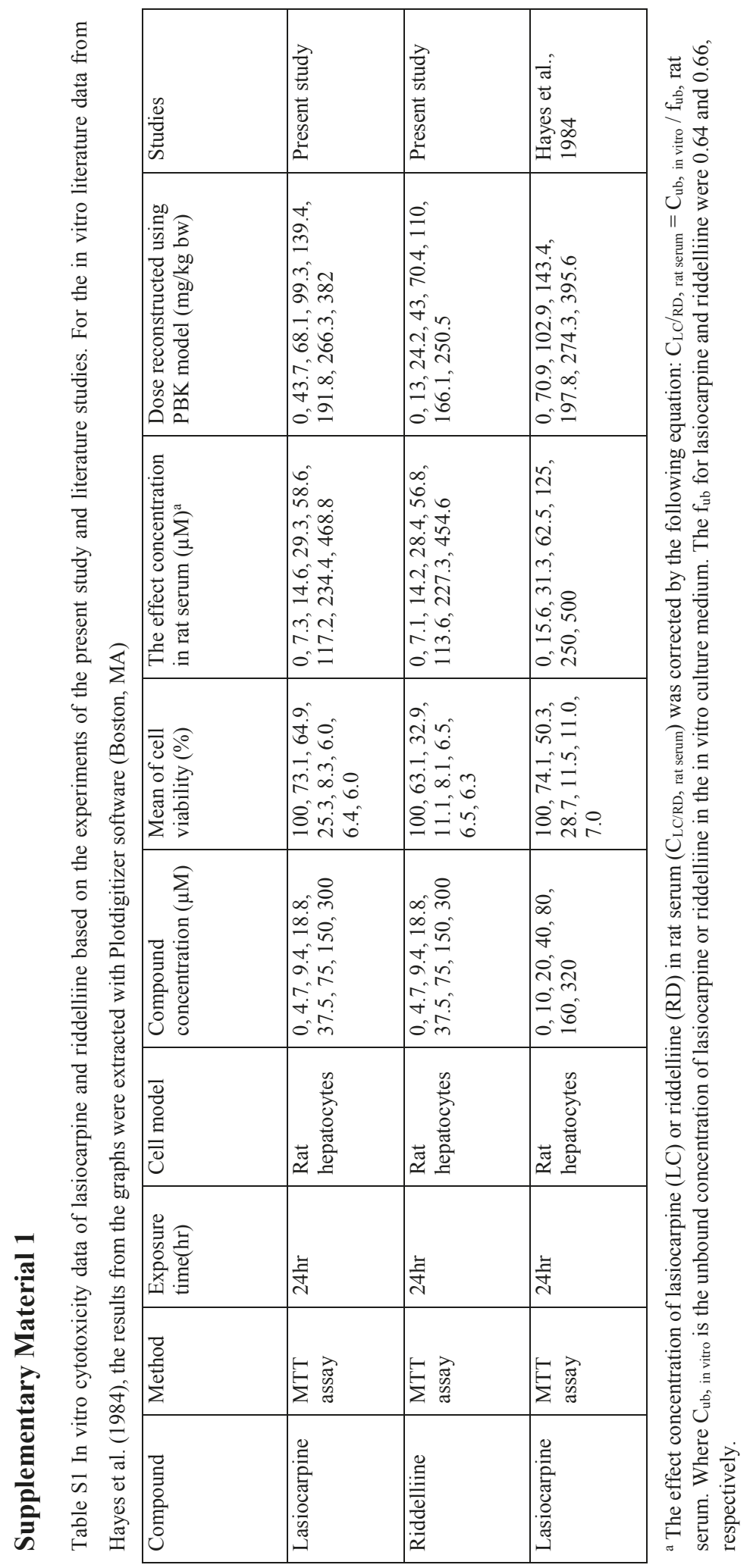


Figure S1 Reported time-dependent blood concentrations (filled square) for riddelliine in mouse and PBK modelling-predicted time-dependent blood concentrations when using Ka value of 0.27/h (black line) (a) or using Ka value of $1.17 / \mathrm{h}$ (dotted line) upon a dose of $10 \mathrm{mg} / \mathrm{kg}$ bw.
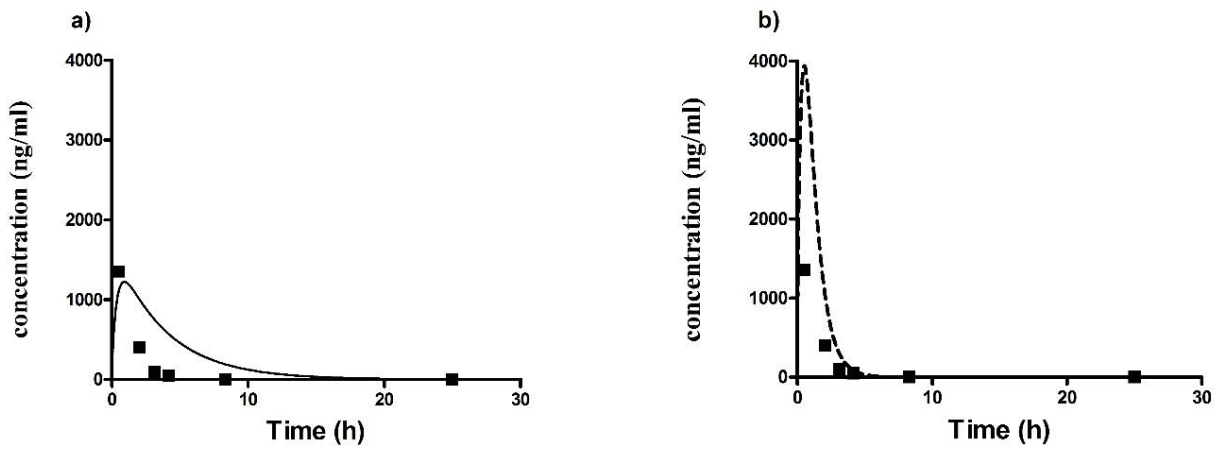

\section{Supplementary Material 2}

PBK Model for lasiocarpine, built with in vitro and in silico data; Species: Rat

;Physiological parameters

;Tissue volumes

$\begin{array}{ll}\mathrm{BW}=0.250\{\mathrm{Kg}\} & ; \text { body weight rat (Brown, } \mathrm{p}) \\ \mathrm{VFc}=0.07 & ; \text { fraction of fat tissue } \\ \mathrm{VLc}=0.034 & ; \text { fraction of liver } \\ \mathrm{VSic}=0.014 & ; \text { fraction of small intestine } \\ \mathrm{VAc}=0.0185 & ; \text { fraction of arterial blood: } 0.074 * 1 / 4 \\ \mathrm{VVc}=0.0555 & ; \text { fraction of venous blood: } 0.074 * 3 / 4 \\ \mathrm{VBc}=0.074 & ; \text { Fraction of blood flow to slowly perfused tissue } \\ \mathrm{VRc}=0.09-\mathrm{VLc}-\mathrm{VSic} & \{\text { L or Kg }\} \\ \mathrm{VSc}=0.82-\mathrm{VFc} & ; \text { volume of fat tissue } \\ \mathrm{VF}=\mathrm{VFc} * \mathrm{BW} & \\ \mathrm{VL}=\mathrm{VLc} * \mathrm{BW} & \\ \mathrm{VSi}=\mathrm{VSic} * \mathrm{BW} & \\ \mathrm{VR}=\mathrm{VRc} * \mathrm{BW} & \\ \mathrm{VS}=\mathrm{VSc} * \mathrm{BW} & \\ \mathrm{VA}=\mathrm{VAc} * \mathrm{BW} & \end{array}$




$$
\begin{aligned}
& \mathrm{VV}=\mathrm{VVc} * \mathrm{BW} \\
& \mathrm{VB}=\mathrm{VBc} * \mathrm{BW}
\end{aligned}
$$

;Blood flow rates

$$
\begin{array}{ll}
\mathrm{QC}=15^{*} \mathrm{BW}^{\wedge} 0.74\{\mathrm{~L} / \mathrm{hr}\} & ; \text { Info: } \mathrm{QC}=15^{*} \mathrm{BW}^{\wedge} 0.74 \text { Reference: Brown, } \mathrm{p} \text { and Krishnan, } \mathrm{p} \\
\mathrm{QFc}=0.07 & ; \text { Fraction of blood flow to fat } \\
\mathrm{QLc}=0.25-\mathrm{QSic} & ; \text { Fraction of blood flow to liver } \\
\mathrm{QSic}=0.76 * 0.014 / 0.09 & ; \text { Fraction of blood flow to small intestine } \\
\mathrm{QRc}=0.76-\mathrm{QLc}-\mathrm{QSic} & ; \text { Fraction of blood flow to richly perfused tissue } \\
\mathrm{QSc}=0.24-\mathrm{QFc} & \\
\mathrm{QF}=\mathrm{QFc} * \mathrm{QC}\{\mathrm{L} / \mathrm{hr}\} & \\
\mathrm{QL}=\mathrm{QLc} * \mathrm{QC} & \\
\mathrm{QSi}=\mathrm{QSic} * \mathrm{QC} & \\
\mathrm{QR}=\mathrm{QRc} * \mathrm{QC} & \\
\mathrm{QS}=\mathrm{QSc} * \mathrm{QC}
\end{array}
$$

;Partition Coefficients

$\begin{array}{ll}\text { lasiocarpine } & \\ \text { PLL }=0.88 & ; \text { liver/blood partition coefficient } \\ \text { PFL }=2.44 & ; \text { fat/blood partition coefficient } \\ \text { PRL }=0.88 & \text {; richly perfused tissues/blood partition coefficient } \\ \text { PSL }=0.48 & \text {; intestine/blood partition coefficient }\end{array}$

;Biochemical parameters

Linear uptake rate $(\mathrm{hr}-1)$

$\mathrm{Ka}=1.55$

;Metabolism liver

;Scaling factors

$\mathrm{MPL}=35$

;Liver microsomal protein yield (mg/gram liver) Atio et al. 1976 
;metabolites of lasiocarpine, unscaled maximum rate of metabolism (nmol min-1 (mg protein)-1)

VmaxLM1c $=5.267 \quad$; based on substrate depletion in rat liver microsome

;metabolites of lasiocarpine, scaled maximum rate of metabolism (umol hr-1)

VMaxLM1 $=$ VMaxLM1c/1000*60*MPL*L*BW

;metabolites of lasiocarpine, affinity constants (umol/L)

KmLM1 $=19.54 \quad$; based on substrate depletion in rat liver microsome

;Metabolism small intestine

;Scaling factors

$\mathrm{MPSi}=20.6$

;Small intestine microsomal fraction yield (uL/gram small intestine) , rung, 2017

$\mathrm{SI}=\mathrm{VSiC}^{*} 1000$

;metabolites of lasiocarpine, unscaled maximum rate of metabolism (nmol min-1 (mg)-1)

VmaxSiM2c $=1.736$; based on adding all the metabolites in the rat intestine microsome ;metabolites of lasiocarpine, scaled maximum rate of metabolism (umol hr-1)

$\mathrm{VMaxSiM} 2=\mathrm{VMaxSiM} 2 \mathrm{c} / 1000 * 60 * \mathrm{MPSi} * \mathrm{SI} * \mathrm{BW}$

;metabolites of lasiocarpine, affinity constants (umol/L)

$\mathrm{KmSiM} 2=23.42 \quad$; based on adding all the metabolites in the rat intestine microsome

;Run settings

Molecular weight

$\mathrm{MWL}=411.2$

;Molecular weight lasiocarpine

;Given dose (mg/ kg bw) and oral dose umol/ kg bw
GDOSE $=10 \quad\{\mathrm{mg} / \mathrm{kg} \mathrm{bw}\}$
; GDOSE $=$ given dose
ODOSE $=$ GDOSE $* 1 \mathrm{E}-3 / \mathrm{MWL} * 1 \mathrm{E} 6\{\mathrm{umol} / \mathrm{kg}$ bw
; ODOSE = given dose recalculated to $\mathrm{umol} / \mathrm{kg}$ bw
DOSE $=$ ODOSE $* \mathrm{BW}$;
; DOSE = umol
;Time
Starttime $=0$
;in hrs
Stoptime $=24$
;in hrs

;Dynamics

slowly perfused tissue compartment 
;AS $=$ Amount lasiocarpine in slowly perfused tissue, umol

$$
\begin{aligned}
& \mathrm{AS}^{\prime}=\mathrm{QS} *(\mathrm{CB}-\mathrm{CVS}) \\
& \text { Init } \mathrm{AS}=0 \\
& \mathrm{CS}=\mathrm{AS} / \mathrm{VS} \\
& \mathrm{CVS}=\mathrm{CS} / \mathrm{PSL}
\end{aligned}
$$

;richly perfused tissue compartment

$; \mathrm{AR}=$ Amount lasiocarpine in richly perfused tissue, umol

$$
\begin{aligned}
& \mathrm{AR}^{\prime}=\mathrm{QR}^{*}(\mathrm{CB}-\mathrm{CVR}) \\
& \text { Init } \mathrm{AR}=0 \\
& \mathrm{CR}=\mathrm{AR} / \mathrm{VR} \\
& \mathrm{CVR}=\mathrm{CR} / \mathrm{PRL}
\end{aligned}
$$

\section{;fat compartment}

$; \mathrm{AF}=$ Amount lasiocarpine in fat tissue, umol

$$
\begin{aligned}
& \mathrm{AF}^{\prime}=\mathrm{QF}^{*}(\mathrm{CB}-\mathrm{CVF}) \\
& \text { Init } \mathrm{AF}=0 \\
& \mathrm{CF}=\mathrm{AF} / \mathrm{VF} \\
& \mathrm{CVF}=\mathrm{CF} / \mathrm{PFL}
\end{aligned}
$$

;uptake lasiocarpine from GI tract

;AGI $=$ Amount lasiocarpine remaining in GI tract (umol)

$$
\begin{aligned}
& \text { AGI' =-ka*AGI } \\
& \text { Init AGI = dose }
\end{aligned}
$$

\section{;liver compartment}

;AL $=$ Amount lasiocarpine in liver tissue, umol

$$
\begin{aligned}
& \mathrm{AL}^{\prime}=\mathrm{QL} * \mathrm{CB}+\mathrm{QSi}{ }^{*} \mathrm{CVSi}-(\mathrm{QL}+\mathrm{QSi}) * \mathrm{CVL}-\mathrm{AMLM} 1 \\
& \text { Init } \mathrm{AL}=0 \\
& \mathrm{CL}=\mathrm{AL} / \mathrm{VL} \\
& \mathrm{CVL}=\mathrm{CL} / \mathrm{PLL}
\end{aligned}
$$

;AMLM1=Amount lasiocarpine metabolized in liver to metabolite 1, umol

$$
\begin{aligned}
& \text { AMLM1' }=\text { VmaxLM1 }{ }^{*} \mathrm{CVL} /(\mathrm{KmLM} 1+\mathrm{CVL}) \\
& \text { Init AMLM1 }=0
\end{aligned}
$$


;small intestine compartment

;ASi $=$ Amount lasiocarpine in small intestine tissue,

$$
\begin{aligned}
& \mathrm{ASi}^{\prime}=\mathrm{QSi}(\mathrm{CB}-\mathrm{CVSi})+\mathrm{ka} * \mathrm{AGI}-\mathrm{AMSiM} 2^{\prime} \\
& \text { Init } \mathrm{ASi}=0 \\
& \mathrm{CSi}=\mathrm{ASi} / \mathrm{VSi} \\
& \mathrm{CVSi}=\mathrm{CSi} / \mathrm{PIL}
\end{aligned}
$$

;AMSiM2=Amount lasiocarpine metabolized in small intestine to metabolites2, umol

$$
\text { AMSiM2' }=\text { VmaxSiM2*CVSi/(KmSiM2 + CVSi) }
$$

Init AMSiM2 $=0$

; arterial blood compartment

;CA $=$ Concentration arterial blood lasiocarpine

$; \mathrm{CA}=\mathrm{CV}$

;venous blood compartment

$; \mathrm{CB}=$ Concentration venous blood lasiocarpine (umol/L)

$\mathrm{AB}^{\prime}=(\mathrm{QF} * \mathrm{CVF}+(\mathrm{QL}+\mathrm{QSi}) * \mathrm{CVL}+\mathrm{QR} * \mathrm{CVR}+\mathrm{QS} * \mathrm{CVS}-\mathrm{QC} * \mathrm{CB})$

Init $\mathrm{AB}=0$

$\mathrm{CB}=\mathrm{AB} / \mathrm{VB}$

$\mathrm{AUC}^{\prime}=\mathrm{CB}$

init $\mathrm{AUC}=0$

Mass balance calculations

\{Mass Balance\}

Total $=$ DOSE

Calculated $=\mathrm{AF}+\mathrm{AS}+\mathrm{AR}+\mathrm{AL}+\mathrm{AB}+\mathrm{AGI}+\mathrm{ASI}+\mathrm{AMLM} 1+\mathrm{AMSiM} 2$

ERROR $=(($ Total-Calculated $) /$ Total $+1 \mathrm{E}-30) * 100$

MASSBBAL $=$ Total-Calculated +1

;blood concentration in $\mathrm{ng} / \mathrm{ml}$

$\mathrm{CBngmL}=\mathrm{CB} * \mathrm{MWL}$ 
PBK Model for riddelliine, built with in vitro and in silico data; Species: Rat

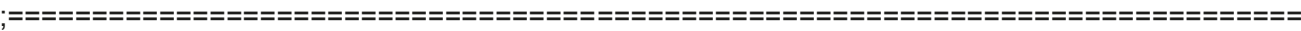

;Physiological parameters

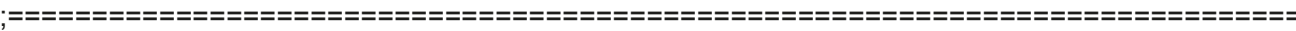

;Tissue volumes

\begin{tabular}{|c|c|}
\hline $\mathrm{BW}=0.250\{\mathrm{Kg}\}$ & ; body weight rat (Brown, p) \\
\hline $\mathrm{VFc}=0.07$ & ; fraction of fat tissue \\
\hline $\mathrm{VLc}=0.034$ & ; fraction of liver \\
\hline $\mathrm{VSic}=0.014$ & ; fraction of small intestine \\
\hline $\mathrm{VAc}=0.0185$ & ; fraction of arterial blood: $0.074 * 1 / 4$ \\
\hline $\mathrm{VVc}=0.0555$ & ; fraction of venous blood: $0.074 * 3 / 4$ \\
\hline \multicolumn{2}{|l|}{$\mathrm{VBc}=0.074$} \\
\hline $\mathrm{VRc}=0.09-\mathrm{VLc}-\mathrm{VSic}$ & ; fraction of richly perfused tissue \\
\hline $\mathrm{VSc}=0.82-\mathrm{VFc}$ & ; Fraction of blood flow to slowly perfused tissue \\
\hline \multicolumn{2}{|l|}{$\mathrm{VF}=\mathrm{VFc} * \mathrm{BW} \quad\{\mathrm{L}$ or $\mathrm{Kg}\}$} \\
\hline $\mathrm{VL}=\mathrm{VLc} * \mathrm{BW}$ & ; Liver volume \\
\hline \multicolumn{2}{|l|}{$\mathrm{VSi}=\mathrm{VSic} * \mathrm{BW}$} \\
\hline \multicolumn{2}{|l|}{$\mathrm{VR}=\mathrm{VRc} * \mathrm{BW}$} \\
\hline \multicolumn{2}{|l|}{$\mathrm{VS}=\mathrm{VSc} * \mathrm{BW}$} \\
\hline \multicolumn{2}{|l|}{$\mathrm{VA}=\mathrm{VAc} * \mathrm{BW}$} \\
\hline $\mathrm{VV}=\mathrm{VVc} * \mathrm{BW}$ & \\
\hline $\mathrm{VB}=\mathrm{VBc} * \mathrm{BW}$ & \\
\hline
\end{tabular}

;Blood flow rates

$\mathrm{QC}=15 * \mathrm{BW}^{\wedge} 0.74\{\mathrm{~L} / \mathrm{hr}\}$

; Info: $\mathrm{QC}=15 * \mathrm{BW}^{\wedge} 0.74$ Reference: Brown, $\mathrm{p}$ and Krishnan, $\mathrm{p}$

$\mathrm{QFc}=0.07$

; Fraction of blood flow to fat

$\mathrm{QLc}=0.25-\mathrm{QSic}$

; Fraction of blood flow to liver

$\mathrm{QSic}=0.76 * 0.014 / 0.09$

; Fraction of blood flow to small intestine

$\mathrm{QRc}=0.76-\mathrm{QLc}-\mathrm{QSic}$

; Fraction of blood flow to richly perfused tissue

$\mathrm{QSc}=0.24-\mathrm{QFc}$

; Fraction of blood flow to slowly perfused tissue

$$
\begin{aligned}
& \mathrm{QF}=\mathrm{QFc} * \mathrm{QC}\{\mathrm{L} / \mathrm{hr}\} \\
& \mathrm{QL}=\mathrm{QLc} * \mathrm{QC} \\
& \mathrm{QSi}=\mathrm{QSic} * \mathrm{QC} \\
& \mathrm{QR}=\mathrm{QRc}^{*} \mathrm{QC} \\
& \mathrm{QS}=\mathrm{QSc} * \mathrm{QC}
\end{aligned}
$$

;Partition Coefficients

;Riddelliine 

$\mathrm{PLL}=0.77$
;liver/blood partition coefficient
$\mathrm{PFL}=0.44$
; fat/blood partition coefficient
$\mathrm{PRL}=0.77$
; richly perfused tissues/blood partition coefficient
$\mathrm{PSL}=0.43$
; slowly perfused tissues/blood partition coefficient
$\mathrm{PIL}=0.77$
; intestine/blood partition coefficien

;Biochemical parameters

;Linear uptake rate (hr-1)

$\mathrm{Ka}=0.72$

;Metabolism liver

;Scaling factors

$\mathrm{MPL}=35$

;Liver microsomal protein yield (mg/gram liver) Atio et al. 1976

$\mathrm{L}=\mathrm{VLC} * 1000$

;Liver $=34($ gram $/ \mathrm{kg} \mathrm{BW})$

;metabolites of riddelliine, unscaled maximum rate of metabolism (nmol min-1 (mg protein)-1)

VmaxLM1c $=2.093 \quad$;based on substrate depletion in rat liver microsome

;metabolites of riddelliine, scaled maximum rate of metabolism (umol hr-1)

VMaxLM1 $=$ VMaxLM1c/1000*60*MPL*L*BW

;metabolites of riddelliine, affinity constants (umol/L)

KmLM1 = 75.69 ;based on substrate depletion in rat liver microsome

;Metabolism small intestine

;Scaling factor

$\mathrm{MPSi}=20.6$

;Small intestine microsome fraction yield (mg/g small intestine), Rung 2017

$\mathrm{Si}=\mathrm{VSiC}^{*} 1000$

;metabolites of riddelliine, unscaled maximum rate of metabolism (nmol min-1 (mg)-1)

VmaxSiM2c $=0.1182 \quad$;based on the substrate depletion intestine microsome

;metabolites of riddelliine, scaled maximum rate of metabolism (umol hr-1)

VMaxSiM2 $=$ VMaxSiM2c $/ 1000 * 60 * M P S i * S I * B W$

;metabolites of riddelliine, affinity constants (umol/L)

$\mathrm{KmSiM} 2=221 \quad$; based on the substrate depletion on intestine microsome

;Run settings

;Molecular weight

MWL =349.38;

;Molecular weight riddelliine

;Given dose (mg/ kg bw) and oral dose umol/ kg bw

GDOSE $=10\{\mathrm{mg} / \mathrm{kg}$ bw $\}$

ODOSE $=$ GDOSE $* 1 \mathrm{E}-3 / \mathrm{MWL} * 1 \mathrm{E} 6 \quad \mathrm{umol} / \mathrm{kg} \mathrm{bw}\}$

DOSE $=$ ODOSE $*$ BW

;GDOSE $=$ given dose

$; \mathrm{ODOSE}=$ given dose recalculated to $\mathrm{umol} / \mathrm{kg}$ bw

; DOSE = umol 
;Time

Starttime $=0$

;in hrs

Stoptime $=24$

;in hrs

;Dynamics

;slowly perfused tissue compartment

;AS = Amount riddelliine in slowly perfused tissue, umol

$\mathrm{AS}^{\prime}=\mathrm{QS}^{*}(\mathrm{CB}-\mathrm{CVS})$

Init $\mathrm{AS}=0$

$\mathrm{CS}=\mathrm{AS} / \mathrm{VS}$

$\mathrm{CVS}=\mathrm{CS} / \mathrm{PSL}$

;richly perfused tissue compartment

;AR $=$ Amount riddelliine in richly perfused tissue, umol

$\mathrm{AR}^{\prime}=\mathrm{QR}^{*}(\mathrm{CB}-\mathrm{CVR})$

Init $\mathrm{AR}=0$

$\mathrm{CR}=\mathrm{AR} / \mathrm{VR}$

$\mathrm{CVR}=\mathrm{CR} / \mathrm{PRL}$

;fat compartment

$; \mathrm{AF}=$ Amount riddelliine in fat tissue, umol

$\mathrm{AF}^{\prime}=\mathrm{QF}^{*}(\mathrm{CB}-\mathrm{CVF})$

Init $\mathrm{AF}=0$

$\mathrm{CF}=\mathrm{AF} / \mathrm{VF}$

$\mathrm{CVF}=\mathrm{CF} / \mathrm{PFL}$

;uptake riddelliine from GI tract

;AGI = Amount riddelliine remaining in GI tract (umol)

AGI' $=-\mathrm{ka}^{*} \mathrm{AGI}$

Init $\mathrm{AGI}=$ dose

;liver compartment

$; \mathrm{AL}=$ Amount riddelliine in liver tissue, umol

$\mathrm{AL}^{\prime}=\mathrm{QL} * \mathrm{CB}+\mathrm{QSi}{ }^{*} \mathrm{CVSi}-(\mathrm{QL}+\mathrm{QSi}) * \mathrm{CVL}-\mathrm{AMLM}{ }^{\prime}$

Init $\mathrm{AL}=0$

$\mathrm{CL}=\mathrm{AL} / \mathrm{VL}$

$\mathrm{CVL}=\mathrm{CL} / \mathrm{PLL}$

;AMLM1=Amount riddelliine metabolized in liver to metabolite 1, umol

AMLM1' $=$ VmaxLM1*CVL/(KmLM1 + CVL $)$

Init AMLM1 $=0$ 
;small intestine compartment

;ASi $=$ Amount riddelliine in small intestine tissue, umol

$$
\begin{aligned}
& \mathrm{ASi}^{\prime}=\mathrm{QSi}{ }^{*}(\mathrm{CB}-\mathrm{CVSi})+\mathrm{ka} * \mathrm{AGI}-\mathrm{AMSiM} 2^{\prime} \\
& \mathrm{Init} \mathrm{ASi}=0 \\
& \mathrm{CSi}=\mathrm{ASi} / \mathrm{VSi} \\
& \mathrm{CVSi}=\mathrm{CSi} / \mathrm{PIL}
\end{aligned}
$$

;AMSiM2=Amount riddelliine metabolized in small intestine to metabolites2, umol

AMSiM2'= VmaxSiM2*CVSi/(KmSiM2 + CVSi $)$

Init $\mathrm{AMSiM} 2=0$

; arterial blood compartment

$; \mathrm{CA}=$ Concentration arterial blood riddelliine

$; \mathrm{CA}=\mathrm{CV}$

; venous blood compartment

; $\mathrm{CB}=$ Concentration venous blood riddelliine (umol/L)

$$
\begin{aligned}
& \mathrm{AB}^{\prime}=(\mathrm{QF} * \mathrm{CVF}+(\mathrm{QL}+\mathrm{QSi}) * \mathrm{CVL}+\mathrm{QR} * \mathrm{CVR}+\mathrm{QS} * \mathrm{CVS}-\mathrm{QC} * \mathrm{CB}) \\
& \text { Init } \mathrm{AB}=0 \\
& \mathrm{CB}=\mathrm{AB} / \mathrm{VB} \\
& \mathrm{AUC}=\mathrm{CB} \\
& \text { init } \mathrm{AUC}=0
\end{aligned}
$$

;Mass balance calculations

\{Mass Balance\}

Total $=$ DOSE

Calculated $=\mathrm{AF}+\mathrm{AS}+\mathrm{AR}+\mathrm{AL}+\mathrm{AB}+\mathrm{AGI}+\mathrm{ASI}+\mathrm{AMLM} 1+\mathrm{AMSiM} 2$

$\mathrm{ERROR}=(($ Total- Calculated $) /$ Total $+1 \mathrm{E}-30) * 100$

MASSBBAL $=$ Total-Calculated +1

; blood concentration in $\mathrm{ng} / \mathrm{ml}$

$\mathrm{CBngmL}=\mathrm{CB} * \mathrm{MWL}$ 
PBK Model for riddelliine, built with in vitro and in silico data; Species: Mouse

;Physiological parameters

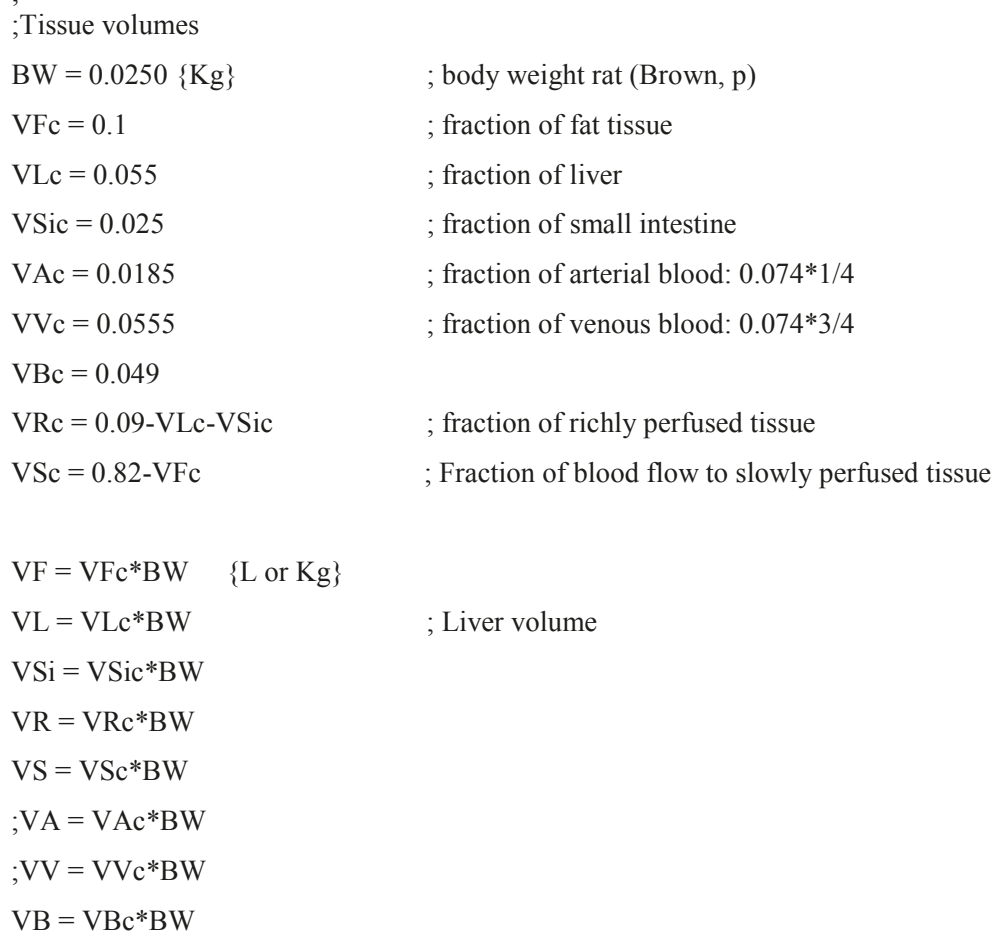

;Blood flow rates

$$
\begin{array}{ll}
\mathrm{QC}=15 * \mathrm{BW} \wedge 0.74\{\mathrm{~L} / \mathrm{hr}\} & ; \text { Info: } \mathrm{QC}=15^{*} \mathrm{BW}^{\wedge} 0.74 \text { Reference: Brown, } \mathrm{p} \text { and Krishnan, } \mathrm{p} \\
\mathrm{QFc}=0.09 & ; \text { Fraction of blood flow to fat } \\
\mathrm{QLc}=0.25-\mathrm{QSic} & ; \text { Fraction of blood flow to liver } \\
\mathrm{QSic}=0.183 & ; \text { Fraction of blood flow to small intestine } \\
\mathrm{QRc}=0.76-\mathrm{QLc}-\mathrm{QSic} & ; \text { Fraction of blood flow to richly perfused tissue } \\
\mathrm{QSc}=0.24-\mathrm{QFc} & ; \text { Fraction of blood flow to slowly perfused tissue } \\
\mathrm{QF}=\mathrm{QFc} * \mathrm{QC}\{\mathrm{L} / \mathrm{hr}\} & \\
\mathrm{QL}=\mathrm{QLc} * \mathrm{QC} & \\
\mathrm{QSi}=\mathrm{QSic} * \mathrm{QC} & \\
\mathrm{QR}=\mathrm{QRc} * \mathrm{QC} & \\
\mathrm{QS}=\mathrm{QSc} * \mathrm{QC}
\end{array}
$$

;Partition Coefficients 
;Riddelliine
PLL $=0.77$
; liver/blood partition coefficient
$\mathrm{PFL}=0.44$
; fat/blood partition coefficient
$\mathrm{PRL}=0.77$
; richly perfused tissues/blood partition coefficient
$\mathrm{PSL}=0.43$
; slowly perfused tissues/blood partition coefficient
$\mathrm{PIL}=0.77$
; intestine/blood partition coefficient

;Biochemical parameters

;Linear uptake rate (hr-1)

$\mathrm{Ka}=0.72$;

;Metabolism liver

;Scaling factors

MPL $=35$

;Liver microsomal protein yield (mg/gram liver) Atio et al. 1976

$\mathrm{L}=\mathrm{VLC} * 1000$

$;$ Liver $=34($ gram $/ \mathrm{kg} \mathrm{BW})$

;metabolites of riddelliine, unscaled maximum rate of metabolism (nmol min-1 (mg protein)-1)

VmaxLM1c $=1.427 \quad$;based on substrate depletion in rat liver microsome

;metabolites of riddelliine, scaled maximum rate of metabolism (umol hr-1)

VMaxLM1 $=$ VMaxLM1 $/ 1000 * 60 *$ MPL $*$ L*BW

;metabolites of riddelliine, affinity constants (umol/L)

$\mathrm{KmLM1}=101.3 \quad$; based on substrate depletion in rat liver microsome

;Metabolism small intestine

;Scaling factor

MPSi=20.6 ;Small intestine microsome fraction yield (mg/g small intestine), Rung 2017

$\mathrm{Si}=\mathrm{VSiC}^{*} 1000$

;metabolites of riddelliine, unscaled maximum rate of metabolism (nmol min-1 (mg)-1)

$\operatorname{VmaxSiM2c}=1.619 \quad$; based on the substrate depletion intestine microsome

;metabolites of riddelliine, scaled maximum rate of metabolism (umol hr-1)

VMaxSiM2 $=$ VMaxSiM2c $/ 1000 * 60 * M P S i * S I * B W$

;metabolites of riddelliine, affinity constants (umol/L)

$\mathrm{KmSiM} 2=695.8$; based on the substrate depletion on intestine microsome

;Run settings

;Molecular weight

MWL $=349.38 \quad ;$ Molecular weight riddelliine

;Given dose (mg/ kg bw) and oral dose umol/ kg bw 


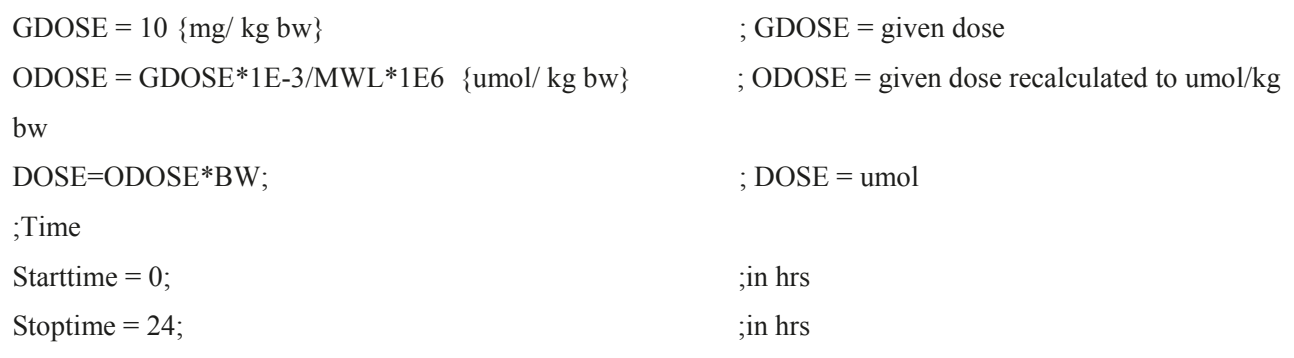

;Dynamics

;slowly perfused tissue compartment

;AS $=$ Amount riddelliine in slowly perfused tissue, umol

$$
\begin{aligned}
& \mathrm{AS}^{\prime}=\mathrm{QS}^{*}(\mathrm{CB}-\mathrm{CVS}) \\
& \text { Init } \mathrm{AS}=0 \\
& \mathrm{CS}=\mathrm{AS} / \mathrm{VS} \\
& \mathrm{CVS}=\mathrm{CS} / \mathrm{PSL}
\end{aligned}
$$

;richly perfused tissue compartment

$; \mathrm{AR}=$ Amount riddelliine in richly perfused tissue, umol

$$
\begin{aligned}
& \mathrm{AR}^{\prime}=\mathrm{QR}^{*}(\mathrm{CB}-\mathrm{CVR}) \\
& \text { Init } \mathrm{AR}=0 \\
& \mathrm{CR}=\mathrm{AR} / \mathrm{VR} \\
& \mathrm{CVR}=\mathrm{CR} / \mathrm{PRL}
\end{aligned}
$$

;fat compartment

$; \mathrm{AF}=$ Amount riddelliine in fat tissue, umol

$$
\begin{aligned}
& \mathrm{AF}^{\prime}=\mathrm{QF}^{*}(\mathrm{CB}-\mathrm{CVF}) \\
& \text { Init } \mathrm{AF}=0 \\
& \mathrm{CF}=\mathrm{AF} / \mathrm{VF} \\
& \mathrm{CVF}=\mathrm{CF} / \mathrm{PFL}
\end{aligned}
$$

;uptake riddelliine from GI tract

;AGI = Amount riddelliine remaining in GI tract (umol)

$$
\begin{aligned}
& \text { AGI' =-ka*AGI } \\
& \text { Init AGI = dose }
\end{aligned}
$$

;liver compartment

$; \mathrm{AL}=$ Amount riddelliine in liver tissue, umol

$$
\mathrm{AL}^{\prime}=\mathrm{QL} * \mathrm{CB}+\mathrm{QSi}{ }^{*} \mathrm{CVSi}-(\mathrm{QL}+\mathrm{QSi}) * \mathrm{CVL}-\mathrm{AMLM}{ }^{\prime}
$$


Init $\mathrm{AL}=0$

$\mathrm{CL}=\mathrm{AL} / \mathrm{VL}$

$\mathrm{CVL}=\mathrm{CL} / \mathrm{PLL}$

;AMLM1=Amount riddelliine metabolized in liver to metabolite 1, umol

AMLM1' $=$ VmaxLM1*CVL/(KmLM1 + CVL)

Init AMLM1 $=0$

;small intestine compartment

;ASi $=$ Amount riddelliine in small intestine tissue, umol

$\mathrm{ASi}^{\prime}=\mathrm{QSi}{ }^{*}(\mathrm{CB}-\mathrm{CVSi})+\mathrm{ka} * \mathrm{AGI}-\mathrm{AMSiM}{ }^{\prime}$

Init $\mathrm{ASi}=0$

$\mathrm{CSi}=\mathrm{ASi} / \mathrm{VSi}$

$\mathrm{CVSi}=\mathrm{CSi} / \mathrm{PIL}$

;AMSiM2=Amount riddelliine metabolized in small intestine to metabolites2, umol

AMSiM2' $=$ VmaxSiM2*CVSi/(KmSiM2 + CVSi $)$

Init $\mathrm{AMSiM} 2=0$

; arterial blood compartment

$; \mathrm{CA}=$ Concentration arterial blood riddelliine

; $\mathrm{CA}=\mathrm{CV}$

; venous blood compartment

$; \mathrm{CB}=$ Concentration venous blood riddelliine (umol/L)

$$
\begin{aligned}
& \mathrm{AB}^{\prime}=(\mathrm{QF} * \mathrm{CVF}+(\mathrm{QL}+\mathrm{QSi}) * \mathrm{CVL}+\mathrm{QR} * \mathrm{CVR}+\mathrm{QS} * \mathrm{CVS}-\mathrm{QC} * \mathrm{CB}) \\
& \text { Init } \mathrm{AB}=0 \\
& \mathrm{CB}=\mathrm{AB} / \mathrm{VB} \\
& \mathrm{AUC} C^{\prime}=\mathrm{CB} \\
& \text { init } \mathrm{AUC}=0
\end{aligned}
$$

;Mass balance calculations

\{Mass Balance\}

Total $=$ DOSE

Calculated $=\mathrm{AF}+\mathrm{AS}+\mathrm{AR}+\mathrm{AL}+\mathrm{AB}+\mathrm{AGI}+\mathrm{ASI}+\mathrm{AMLM} 1+\mathrm{AMSiM} 2$

ERROR $=(($ Total - Calculated $) /$ Total $+1 \mathrm{E}-30) * 100$

MASSBBAL $=$ Total-Calculated +1

; blood concentration in $\mathrm{ng} / \mathrm{ml}$

$\mathrm{CBngmL}=\mathrm{CB} * \mathrm{MWL}$ 


\section{Chapter 5}

\section{Use of an in vitro-in silico testing strategy to predict inter- species and inter-ethnic human differences in liver toxicity of the pyrrolizidine alkaloids lasiocarpine and riddelliine}

Jia Ning, Lu Chen, Marije Strikwold, Jochem Louisse, Sebastiaan Wesseling, Ivonne M.C.M. Rietjens

Published in: Archives of Toxicology, (2019) 93, 801-818 


\begin{abstract}
Lasiocarpine and riddelliine are pyrrolizidine alkaloids (PAs) known to cause liver toxicity. The aim of this study was to predict the inter-species and inter-ethnic human differences in acute liver toxicity of lasiocarpine and riddelliine using physiologically based kinetic (PBK) modelling based reverse dosimetry of in vitro toxicity data. The concentration-response curves of in vitro cytotoxicity of lasiocarpine and riddelliine defined in pooled human hepatocytes were translated to in vivo doseresponse curves by PBK models developed using kinetic data obtained from incubations with pooled tissue fractions from Chinese and Caucasian individuals, providing PBK models for the average Chinese and average Caucasian, respectively. From the predicted in vivo dose-response curves, the benchmark dose lower and upper confidence limits for $5 \%$ effect $\left(\mathrm{BMDL}_{5}\right.$ and $\left.\mathrm{BMDU}_{5}\right)$ were derived and subsequently compared to those previously obtained in rat to evaluate inter-species differences. The inter-species differences amounted to 2.0-fold for lasiocarpine and 8.2-fold for riddelliine with humans being more sensitive than rats. The inter-ethnic human differences varied 2.0-fold for lasiocarpine and 5.0-fold for riddelliine with the average Caucasian being more sensitive than the average Chinese. In conclusion, the present study provides the proof-of-principle to predict interspecies and inter-ethnic differences in in vivo liver toxicity for PAs by an alternative testing strategy integrating in vitro cytotoxicity data with PBK modelling-based reverse dosimetry.
\end{abstract}




\section{Introduction}

Lasiocarpine and riddelliine (Figure 1) are chemicals belonging to the group of pyrrolizidine alkaloids (PAs) which are important secondary metabolites of approximately 6000 plant species distributed around the world (Smith and Culvenor, 1981). Lasiocarpine is widely distributed in the Heliotropium and Symphytum genus (Boraginaceae species), and riddelliine is present in the genus Senecio (Asteraceae species) (Adams et al., 1942; Smith and Culvenor, 1981). PA-containing plants can be toxic to livestock, wildlife and humans (Stegelmeier et al., 1999). Human exposure to lasiocarpine and riddelliine may result from the consumption of contaminated food staples, plant food supplements, herbal medicines, herbal teas, honey and milk (EFSA, 2011, 2017). Exposure to lasiocarpine, as well as riddelliine has been shown to cause fatal liver veno-occlusive disease in animals and humans (Bane et al., 2012; Mohabbat et al., 1976; Tandon et al., 1978). A 2-year National Toxicology Program (NTP) study testing the genotoxicity and carcinogenicity of lasiocarpine and riddelliine showed that these PAs are also carcinogenic and genotoxic compounds (Chan et al., 2003; NTP, 1978, 2003). Liver angiosarcomas were observed in 11 of 23 male rats and 7 of 24 female rats following dietary administration of $0.75 \mathrm{mg} / \mathrm{kg}$ bw/day of lasiocarpine. Riddelliine induced a high incidence of liver hemangiosarcoma in both male (43 out of 50 rats) and female rats (38 out of 50 rats) at $0.7 \mathrm{mg} / \mathrm{kg}$ bw/day (Chan et al., 2003; NTP, 1978, 2003). The International Agency for Research on Cancer (IARC) classified these alkaloids in Group 2B, potentially carcinogenic to humans (IARC, 2017).

Following absorption in the gastrointestinal tract, PAs are distributed to the liver where the main metabolism occurs (Fu et al., 2004). In general, three metabolic pathways have been identified for lasiocarpine (heliotridine-type PA) and riddelliine (retronecine-type PA) (Figure 1) (EFSA, 2011; Fu et al., 2004). The bioactivation pathway is the conversion of lasiocarpine or riddelliine into a toxic intermediate, known as a dehydropyrrolizidine alkaloid, in a reaction mainly catalysed by cytochrome P450 3A4 (Fu et al., 2004). Dehydropyrrolizidine alkaloids react with cellular proteins and DNA leading to liver toxicity, genotoxicity and carcinogenicity (Fu et al., 2004). Other metabolic pathways include hydrolysis and N-oxidation, leading to the formation of necine bases and PA N-oxides, respectively (Figure 1). These latter two metabolic pathways are considered to lead to detoxification (EFSA, 2011).

Significant variations between species regarding the toxicity of PAs have been observed. Recently, Fashe et al. (2015) conducted a comparative study on the metabolism of lasiocarpine by human and rat liver microsomes and found that humans might be more susceptible to lasiocarpine-induced toxicity, because the overall metabolic fingerprint detected upon incubation with human liver microsomes differed from that obtained with rat liver microsomes in the following two major aspects: 1) the amount of detoxified metabolites was lower in incubations with human liver microsomes than with rat liver microsomes, and 2) higher levels of GSH-reactive metabolites were formed in incubations with 
the human liver microsomes than with the rat liver microsomes. These species differences may be mediated by interspecies differences in biotransformation capacities including CYP 3A4 catalysed bioactivation, carboxylesterase-mediated hydrolysis and the level of cellular GSH (Fashe et al., 2015). In addition to these possible species differences in biotransformation capacities, other studies reported ethnic differences in P450 dependent metabolism and these may potentially result in inter-ethnic differences in PA bioactivation and detoxification. Yang et al. (2012) reported significant differences in phenacetin $O$-deethylation, diclofenac 4'-hydroxylation, $(S)$-mephenytoin 4'-hydroxylation and chlorzoxazone 6-hydroxylation reactions, mediated by CYP 1A2, CYP 2C9, CYP 2C19 and CYP 2E1, respectively between Chinese and Caucasian liver microsomal preparations. Previously, we also reported differences between Chinese and Caucasians regarding the metabolic bioactivation and detoxification of the food-borne genotoxic carcinogen estragole, resulting in a predicted 4.5 -fold lower formation of the ultimate carcinogenic metabolite of estragole with similar rates of detoxification in Chinese as compared to the Caucasians, and thus a possible lower risk of estragole exposure for the average Chinese at similar levels of exposure (Ning et al., 2017).

Thus, inter-species and inter-ethnic human differences in metabolism might occur and may in theory also affect the sensitivity towards PA toxicity. The aim of this study was to predict the inter-species and inter-ethnic human differences in acute liver toxicity of lasiocarpine and riddelliine using physiologically based kinetic (PBK) modelling-based reverse dosimetry of in vitro toxicity data. Previously, we developed a PBK modelling-based approach to predict acute liver toxicity of lasiocarpine and riddelliine in rats (Chen et al., 2018). The predicted $\mathrm{BMDL}_{5}-\mathrm{BMDU}_{5}$ values for in vivo liver toxicity of lasiocarpine and riddelliine in rats amounted to $23.0-34.4$ and $4.9-8.4 \mathrm{mg} / \mathrm{kg}$ bw/day, respectively (Chen et al., 2018). That study already showed that the PBK modelling-based approach was able to define inter-compound differences in acute liver toxicity of PAs. The study of Culvenor (1983) estimated that a dose of $0.7-3 \mathrm{mg} / \mathrm{kg}$ bw/day of riddelliine may lead to acute liver toxicity in humans, indicating that humans might be more sensitive than rats towards liver toxicity of PAs. In the present study we developed PBK models for lasiocarpine and riddelliine in different ethnic populations (Chinese and Caucasian) and used these models to convert in vitro toxicity data obtained in pooled human hepatocytes to predict in vivo dose-response data and $\mathrm{BMDL}_{5}-\mathrm{BMDU}_{5}$ values for acute liver toxicity of lasiocarpine and riddelliine in humans. Subsequently, these points of departure (PoDs) were compared to PoDs derived previously for rats by the same methodology (Chen et al., 2018) to evaluate the occurrence of species differences in the metabolism and toxicity of lasiocarpine and riddelliine while the PoDs obtained for the average Chinese and average Caucasian provided insight in inter-ethnic differences. 


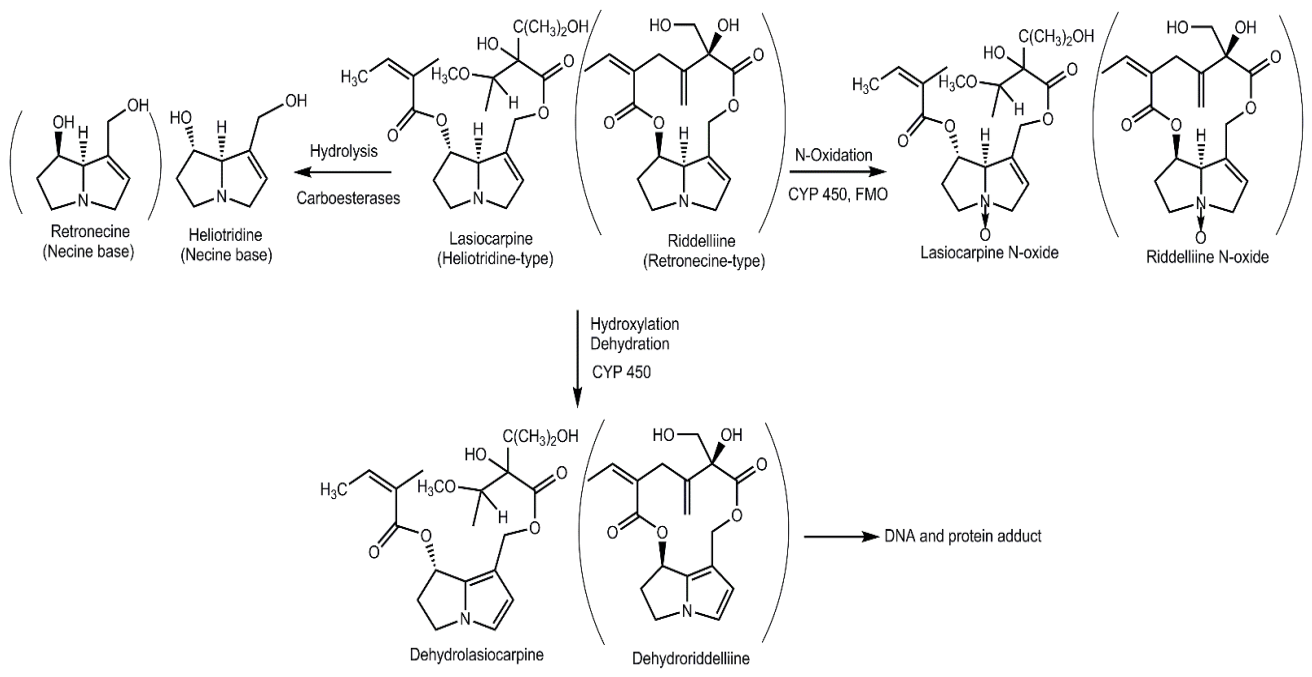

Fig. 1 Schematic presentation of the major metabolic pathways of lasiocarpine (heliotridine-type PA) and riddelliine (retronecine-type PA), the latter shown in brackets. FMO = flavin-containing monooxygenase, CYP $450=$ cytochromes P450.

\section{Materials and Methods}

\section{Chemicals and biological materials}

Lasiocarpine (purity $>97 \%$ ) was purchased from Phytolab (Vestenbergsgreuth, Germany). Riddelliine (purity $>90 \%$ ) was a gift from RIKILT (Wageningen, The Netherlands) and from the National Institute for Environmental Health Sciences (NIEHS) (Durham, USA). The HepaRG cell line was obtained from Biopredic International (Saint-Grégoire, France). Cryopreserved pooled human hepatocytes (5 mixed gender donors), cryopreserved hepatocyte plating medium (CHPM) (CM 9000) and cryopreserved hepatocyte recovery medium (CHRM) (CM 7000) were purchased from Invitrogen (Cheshire, UK). Willliams medium E (without glutamine), penicillin/streptomycine-solution, Lglutamine, phosphate buffered saline (PBS), and trypsin-EDTA were purchased from Gibco (Paisley, Scotland, UK). Fetal bovine serum was purchased from the American Type Culture Collection (ATCC) (Manassas, USA). Dimethylsulfoxide (DMSO), human insulin and hydrocortisone-21-hemisuccinate were purchased from Sigma-Aldrich (Steinheim, Germany). Potassium dihydrogen phosphate, dipotassium hydrogen phosphate trihydrate, sodium phosphate, sodium chloride and trifluoroacetic acid (TFA) were purchased from VWR International (Darmstadt, Germany). Reduced nicotinamide adenine dinucleotide phosphate (NADPH) was obtained from Roche Diagnostics (Mannheim, Germany). Acetonitrile (UPLC/MS grade) was obtained from Biosolve BV (Valkenswaard, Netherlands). Human plasma and rapid equilibrium dialysis (RED) materials, including RED inserts, RED base plate and sealing tape were obtained from Thermo Fisher Scientific (Bleiswijk, the the Netherlands). Pooled Caucasian liver microsomes (33 donors) were purchased from Corning 
(Woburn, MA, USA). Pooled Caucasian intestinal microsomes (15 donors) were obtained from Xenotech (Lenexa, USA). Pooled Caucasian human lung and kidney microsomes were purchased from Biopredic International (Rennes, France). Pooled Chinese liver microsomes (40 donors) were purchased from PrimeTox (Wuhan, China).

\section{Outline of the PBK modelling-based reverse dosimetry approach in human}

The PBK modelling-based reverse dosimetry approach to predict the in vivo dose-response curves for risk assessment from in vitro cytotoxicity concentration-response data included the following steps: 1) establishment of in vitro cytotoxicity concentration-response curves for lasiocarpine and riddelliine in HepaRG cells and in cryopreserved pooled human hepatocytes from Caucasian donors and correcting for toxicity expected in hepatocytes from the Chinese population as described below, development of PBK models for lasiocarpine and riddelliine in human using the kinetic parameters defined in the present study using both Chinese and Caucasian human liver subcellular factions, 3) translation of in vitro concentration-response curves to the in vivo dose-response curves using the PBK models, 4) BMD analysis based on the predicted in vivo dose-response curves to obtain the $\mathrm{BMDL}_{5}$ and $\mathrm{BMDU}_{5}$ values as points of departure (PoDs), 5) comparison of the predicted PoDs in human (Chinese and Caucasian) to one another and to the previously predicted PoDs in rats (Chen et al., 2018) to assess for inter-ethnic and inter-species differences in acute liver toxicity.

\section{In vitro liver toxicity data}

Two types of cells were used to determine the in vitro concentration-response curves for acute cytotoxicity in human liver including cells from the HepaRG cell line and human hepatocytes. The HepaRG cell line is a human hepatoma cell line, which can differentiate into hepatocyte-like morphology by treatment with DMSO (Kanebratt and Andersson, 2008). HepaRG cells were seeded at a density of 9000 cells/well in 96-well plates in growth medium consisting of Williams' medium E, supplemented with $10 \%$ fetal bovine serum (FCS), $100 \mathrm{IU} / \mathrm{ml}$ penicillin, $100 \mu \mathrm{g} / \mathrm{ml}$ streptomycin, $1 \%$ (v/v) L-glutamine, $5 \mu \mathrm{g} / \mathrm{ml}$ human insulin and $50 \mu \mathrm{M}$ hydrocortisone-21-hemisuccinate. The medium was renewed every two to three days. After two weeks, the cells were nearly confluent and were cultured in the differentiation medium supplemented with $1.7 \%(\mathrm{v} / \mathrm{v})$ DMSO. The medium was refreshed every two to three days. After two weeks differentiation treatment, HepaRG cells were fully differentiated and were used for experiments within four weeks with refreshing the medium every two to three days according to the protocol provided by Biopredic International.

Cryopreserved mixed gender primary human hepatocytes from five Caucasian donors were also used to perform the cytotoxicity experiments and define in vitro concentration-response curves. Cryopreserved human hepatocytes were thawed according to the protocol provided by Invitrogen. Briefly, human hepatocytes were thawed in a $37{ }^{\circ} \mathrm{C}$ water bath and transferred to $50 \mathrm{ml}$ thawing medium (CM 7000). The cells were centrifuged at room temperature at $100 \mathrm{~g}$ for $10 \mathrm{~min}$ and resuspended in the plating were seeded in 96-well plates at a density of 50,000 cells/well and incubated 
in a $5 \% \mathrm{CO}_{2}: 95 \%$ air- humidified incubator for $6 \mathrm{~h}$, after which the cells were ready to be exposed to the compounds. Only batches with a cell viability $>90 \%$ were used for experiments.

Cytotoxicity was determined with the MTT assay by assessing the activity of mitochondrial dehydrogenase enzymes according to the method of Field et al. (2015). In brief, the fully differentiated HepaRG cells and human hepatocytes were exposed to serum free medium containing different concentrations of lasiocarpine and riddelliine ranging from 4 to $300 \mu \mathrm{M}$ (final concentrations), added from 200 times concentrated stock solutions in DMSO. Each concentration was measured in triplicate and three independent experiments were performed. A solvent control was included in each independent experiment. After 24 hours exposure, MTT $(0.45 \mathrm{mg} / \mathrm{ml}$ final concentration) was added and cells were incubated for another 2 hours in the incubator. The formed MTT formazan crystals were dissolved in DMSO and the absorbance was measured at a microplate reader at $562 \mathrm{~nm}$ (SpectraMax M2, USA). All the data are presented as mean values \pm SD. The half maximal inhibitory concentration $\left(\mathrm{IC}_{50}\right)$ values used to assess the in vitro acute liver toxicity of lasiocarpine and riddelliine were obtained using GraphPad Prism 5.04 (GraphPad software, San Diego California U.S.A.) using a non-linear regression model for curve fitting $\mathrm{Y}=$ Bottom $+($ Top-Bottom $) /\left(1+10^{\wedge}((\mathrm{X}-\operatorname{LogIC} 50))\right)$. The in vitro concentration-response curve for liver toxicity for Chinese hepatocytes was obtained as described below.

\section{In vitro microsomal incubations}

The in vitro incubation for the conversion of lasiocarpine with Chinese and Caucasian liver microsomes were performed as described by Fashe et al. (2015). Briefly, mixed gender human liver microsomes were incubated with lasiocarpine in the presence of NADPH. The incubation mixtures contained (final concentrations) $2 \mathrm{mM} \mathrm{NADPH}$ and $0.04 \mathrm{mg} / \mathrm{ml}$ microsomal protein in $0.1 \mathrm{M}$ potassium phosphate ( $\mathrm{pH}$ 7.4). After pre-incubation for $5 \mathrm{~min}$, the reaction was started by adding NADPH. Incubations were performed for $30 \mathrm{~min}$ at substrate concentrations ranging from 3 to 200 $\mu \mathrm{M}$ (final concentrations) (added from 100 times concentrated stock solutions in DMSO), after which the reaction was terminated by adding ice-cold acetonitrile $(20 \% \mathrm{v} / \mathrm{v})$. Under these conditions conversion of lasiocarpine was linear in time (1-30 $\mathrm{min}$ ) and with the amount of microsomal protein (0.01-0.04 $\mathrm{mg} / \mathrm{ml}$ ) (data not shown). Blank incubations were performed in the absence of the cofactor NADPH.

The incubation conditions for metabolism of riddelliine with human liver microsomes contained (final concentrations) $2 \mathrm{mM}$ NADPH, $0.5 \mathrm{mg} / \mathrm{ml}$ microsomal protein for Chinese and Caucasian samples in $0.1 \mathrm{M}$ potassium phosphate ( $\mathrm{pH} 7.4$ ) and 3 to $400 \mu \mathrm{M}$ (final concentrations) riddelliine added from 100 times concentrated stock solutions in DMSO. After 5 min pre-incubation at $37{ }^{\circ} \mathrm{C}$, the reactions were started by adding NADPH. Incubations were carried out for $30 \mathrm{~min}$ and the reaction was terminated by adding ice-cold acetonitrile $(20 \% \mathrm{v} / \mathrm{v})$. Under these conditions conversion 
of riddelliine was linear in time (1-30 $\mathrm{min})$ and with the amount of microsomal protein (0.04-0.5 $\mathrm{mg} / \mathrm{ml}$ ) (data not shown). Blank incubations were performed in the absence of the cofactor NADPH.

To compare the inter-ethnic differences in metabolic activation of lasiocarpine and riddelliine, incubations with Chinese and Caucasian liver microsomes were performed as described above in the presence of $4 \mathrm{mM}$ glutathione (GSH) to scavenge dehydropyrrolizidine alkaloids metabolites. The level of GSH in the incubations was optimized to obtain the maximum scavenging of dehydropyrrolizidine alkaloids metabolites as major glutathione conjugate, namely 7-GS-DHP (Tamta et al., 2012), which was analyzed by LC-MS/MS analysis as described below.

The kinetic constants for conversion of lasiocarpine and riddelliine by Caucasian intestinal, lung and kidney microsomal preparations were determined as described above for liver microsomes but increasing the incubation time to $2 \mathrm{~h}$. Under these conditions conversion of lasiocarpine and riddelliine was linear with time (1-120 minutes) and with the amount of microsomal protein added (0.01-0.04 $\mathrm{mg} / \mathrm{ml}$ for lasiocarpine, $0.04-0.5 \mathrm{mg} / \mathrm{ml}$ for riddelliine) for the intestine, whereas negligible conversion was observed for lung and kidney (data not shown). Before Ultra Performance Liquid Chromatography photodiode array analysis (UPLC-PDA) or LC-MS/MS analysis, all samples were centrifuged for $5 \mathrm{~min}$ at $15,000 \mathrm{~g}$ to precipitate microsomal proteins. Intestine, lung and kidney microsomes from the Chinese population are not available. However, the kinetic parameters obtained using pooled intestinal microsomal preparations from Caucasian individuals were used for the Chinese PBK model, because the results of the sensitivity analysis revealed that the parameters related to the intestinal metabolism of lasiocarpine and riddelliine do not have a large influence on the predicted maximum concentration in the liver blood $\left(\mathrm{C}_{\max }\right)$. Because conversion by kidney and lung microsomes was negligible (see result section), no further kinetic data for these organs were required.

The substrate depletion approach was used to obtain the kinetic parameters. The amount of substrate depletion of lasiocarpine and riddelliine was determined as the amount detected in the blank incubations in the absence of the cofactor NADPH minus the amount detected in the incubations with NADPH. The amounts of 7-GS-DHP formed were corrected for the amounts detected in the blank incubations performed without cofactor NADPH. The data for the rate of conversion of lasiocarpine and riddelliine with increasing substrate concentration [S] were fitted to the standard MichaelisMenten equation (1):

(1) $\mathrm{v}=\mathrm{V}_{\max } /\left(1+\mathrm{K}_{\mathrm{m}} /[\mathrm{S}]\right)$

The apparent maximum velocity $\left(\mathrm{V}_{\max }\right)$ and the apparent Michaelis-Menten constant $\left(\mathrm{K}_{\mathrm{m}}\right)$ were determined by fitting the data to this equation using GraphPad Prism version 5.04 (GraphPad software, San Diego California U.S.A.). $\mathrm{V}_{\max }$ values expressed as $\mathrm{nmol} / \mathrm{min} /(\mathrm{mg}$ microsomal protein) were scaled to the $\mathrm{V}_{\max }$ expressed as $\mu \mathrm{mol} / \mathrm{h} / \mathrm{kg}$ bw using microsomal protein yields of $35 \mathrm{mg} / \mathrm{g}$ liver 
(Medinsky et al. 1994) and $20.6 \mathrm{mg} / \mathrm{g}$ small intestine (Cubitt et al. 2009). The $\mathrm{K}_{\mathrm{m}}$ values determined in vitro were assumed to be equal to in vivo $\mathrm{K}_{\mathrm{m}}$ values.

\section{Protein binding correction}

To correct for protein binding in the in vivo situation in human liver blood, while the in vitro toxicity was determined in serum free medium, the fraction of unbound lasiocarpine and riddelliine under the in vivo conditions $\left(\mathrm{f}_{\mathrm{ub}}\right)$ was determined. To this end the $\mathrm{f}_{\mathrm{ub}}$ for both compounds in human blood was determined using the RED (rapid equilibrium dialysis) device. According to the protocol described by Waters et al. (2008), $300 \mu \mathrm{l}$ of spiked human plasma containing $5 \mu \mathrm{M}$ lasiocarpine and 5 or $50 \mu \mathrm{M}$ riddelliine (final concentration, $1 \% \mathrm{v} / \mathrm{v}$ DMSO) were added to the plasma chambers of the RED device insert, while $500 \mu \mathrm{l}$ dialysis buffer (PBS containing $100 \mathrm{mM}$ sodium phosphate and $150 \mathrm{mM}$ sodium chloride) were added to the buffer chamber. The device was sealed with tape and incubated at $37{ }^{\circ} \mathrm{C}$ on a shaker at $250 \mathrm{rpm}$ for $5 \mathrm{~h}$. After the system reached equilibrium, $50 \mu \mathrm{l}$ of post-dialysis samples were collected from the plasma and buffer chambers into separate tubes. Subsequently, $50 \mu \mathrm{l}$ of human plasma was added to the buffer samples and the same volume of dialysis buffer was added to the plasma samples, after which protein was precipitated by addition of ice-cold acetonitrile ( $75 \%$ $\mathrm{v} / \mathrm{v}$ ). After incubation on ice for $30 \mathrm{~min}$, the mixtures were centrifuged at $15,000 \mathrm{~g}$ for $15 \mathrm{~min}$ at $4{ }^{\circ} \mathrm{C}$, and the supernatants were analyzed by UPLC-PDA. The measurements were performed in three independent experiments.

The fraction of unbound lasiocarpine and riddelliine in human blood $\left(f_{\mathrm{ub}}\right)$ was assumed to be the same as in human plasma in the present study and was defined as the ratio of the concentration of the test compound in the buffer chamber and the concentration in the plasma chamber (van Liempd et al., 2011; Waters et al., 2008). The $f_{u b}$ thus obtained was used to correct for the difference in protein binding in the in vitro assay and the in vivo situation when performing the PBK modelling-based reverse dosimetry. To this end the effect concentration of lasiocarpine and riddelliine in human liver blood $\left(\mathrm{C}_{\mathrm{Lc} / \mathrm{Rd} \text {, human blood }}\right)$ used for reverse dosimetry was corrected by the following equation (2):

(2) $\mathrm{C}_{\mathrm{Lc} / \mathrm{Rd} \text {, human blood }}=\mathrm{C}_{\mathrm{ub}}$, in vitro $/ \mathrm{f}_{\mathrm{ub}}$

Where $\mathrm{f}_{\mathrm{ub}}$ is the fraction of the unbound lasiocarpine and riddelliine in human blood and $\mathrm{C}_{\mathrm{ub}}$, in vitro is the unbound concentration of lasiocarpine and riddelliine in the in vitro culture medium. Since the exposure medium for the HepaRG and human hepatocyte cell lines was serum free, the concentrations of lasiocarpine and riddelliine in the in vitro cytotoxicity assay were considered to be the unbound concentration. This correction implies that the unbound fraction in vitro is set equal to the unbound fraction in vivo.

\section{UPLC and LC-MS/MS analysis}

The supernatant of each sample was analyzed on UPLC using a BEH C18 $(1.7 \mu \mathrm{m} 2.1 \times 50 \mathrm{~mm})$ 
column with a guard column and a photodiode array detector (PDA) (Acquity, Waters). The flow rate was $0.6 \mathrm{ml} / \mathrm{min}$ and the mobile phase consisted of ultrapure water with $0.1 \%(\mathrm{v} / \mathrm{v})$ TFA and acetonitrile. For the analysis of lasiocarpine, a gradient was applied from 0 to $50 \%$ acetonitrile in ultrapure water in $2 \mathrm{~min}$, after which the percentage of acetonitrile was increased to $100 \%$ in $3 \mathrm{~min}$ and kept at this level for 2 min longer, after which the column was set back to the starting conditions. Under these conditions, lasiocarpine had a retention time of $1.9 \mathrm{~min}$. For detection of riddelliine, a linear gradient from 0 to $50 \%$ acetonitrile in ultrapure water during 4 min was applied, followed by an increase to $100 \%$ acetonitrile in $1 \mathrm{~min}$. This percentage was kept for $1 \mathrm{~min}$ to wash the column, after which the column was set back to the starting conditions. Under these conditions, riddelliine eluted at $1.8 \mathrm{~min}$. Identification of lasiocarpine and riddelliine was achieved by comparison of the UV spectra and retention times of these compounds to those of reference compounds. Quantification of lasiocarpine and riddelliine was done by comparing the peak areas of these compounds at a wavelength of $220 \mathrm{~nm}$ to the calibration curve of the corresponding standard compounds.

The major glutathione conjugate of lasiocarpine and riddelliine, 7-GS-DHP was analyzed and quantified by LC-MS/MS. LC-MS/MS analysis was performed on a Shimadzu Nexera XR LC-20AD SR UPLC system coupled with a Shimadzu LCMS-8040 mass spectrometer (Kyoto, Japan). The samples $(1 \mu \mathrm{l})$ were loaded onto a reverse phase C18 column $(1.7 \mu \mathrm{m} 2.1 \times 50 \mathrm{~mm})$ with a flow rate of $0.3 \mathrm{ml} / \mathrm{min}$. The column temperature was set to $40{ }^{\circ} \mathrm{C}$. The mobile phase consisted of ultrapure water with $0.1 \%(\mathrm{v} / \mathrm{v})$ formic acid and acetonitrile containing $0.1 \%(\mathrm{v} / \mathrm{v})$ formic acid. The gradient began with $0 \%$ acetonitrile for one minute to wash away unwanted salts, followed by a linear gradient from 0 to $5 \%$ in $8 \mathrm{~min}$ and a further increase to $100 \%$ in $6 \mathrm{~min}$. This percentage was kept for $0.5 \mathrm{~min}$, after which the column was set back to the starting conditions. A Shimadzu LCMS-8040 triple quadrupole with electrospray ionization (ESI) interface was used to perform the MS-MS analysis. The instrument was operated in positive mode in the multiple reaction monitoring (MRM) mode with a spray voltage of $4.5 \mathrm{KV}$. The 7-GS-DHP was monitored at the $[\mathrm{M}+\mathrm{H}]^{+}$of precursor to product $443.2 \rightarrow$ $425.15(\mathrm{CE}=-7 \mathrm{kV}), 443.2 \rightarrow 118.1(\mathrm{CE}=-24 \mathrm{kV})$ and $443.2 \rightarrow 247.2(\mathrm{CE}=-15 \mathrm{kV}) \mathrm{m} / \mathrm{z}$. Some of these MRMs were also used in study of Tamta et al. (2012). Under these conditions, 7-GS-DHP eluted at $8.97 \mathrm{~min}$ and the chromatogram of 7-GS-DHP is presented in the supplementary materials 1 . The peak area of total ion chromatogram (TIC) of 7-GS-DHP was acquired using Postrun analysis in the software LabSolution (Shimadzu). The ratio of the peak areas of 7-GS-DHP formed in incubations with Chinese and Caucasian liver microsomes at each substrate concentration, was assumed to reflect the inter-ethnic differences in metabolic activation of lasiocarpine and riddelliine. Thus these ratios were used to convert the concentration-response curve for in vitro toxicity obtained with human hepatocytes derived from Caucasian donors to a curve for Chinese donors. To this end the percentage of cytotoxicity at each concentration was multiplied by the amount of 7-GS-DHP formed in incubation 
with Caucasian liver samples divided by the amount formed in similar incubations with Chinese liver microsomes. This provided an estimated concentration-response curve for cytotoxicity towards Chinese liver hepatocytes.

\section{Development of a PBK model for lasiocarpine and riddelliine in human}

PBK models for lasiocapine and riddelliine for the average Chinese and average Caucasian were developed based on the PBK models previously defined and evaluated by Chen et al. (2018) for lasiocarpine and riddelliine in rats. The structure of the model for lasiocarpine and riddelliine is presented in Figure 2. The model has 7 compartments including blood, fat, rapidly perfused tissue, slowly perfused tissue, liver and intestine that are mutually connected through the systemic circulation which is represented by a blood compartment. The intestine and liver compartments are included in the model, since the conversion of lasiocarpine and riddelliine occurs in these organs. The physiological parameters for the average Chinese and average Caucasian were derived from the literature (Brown et al., 1997; NHFPC, 2007, 2014) and are presented in Table S2 in the supplementary materials 2 . Several reports from the literature indicate that Chinese have significantly smaller livers than Caucasians (Chan et al., 2006; Shi et al., 2009; Yuan et al., 2008). The physiological parameters provided by National Health Commission of the People's Republic of China (NHFPC) also show that the Chinese liver volume is 1.3-fold lower than the Caucasian liver volume. The tissue:blood partition coefficients were estimated from $\log \mathrm{K}_{\mathrm{ow}}$ according to the method described by DeJongh et al. (1997). The $\log \mathrm{K}_{\mathrm{ow}}$ values for lasiocarpine and riddelliine were estimated by ChemBio 3D 2010 software (CambrigeSoft, USA).

Lasiocarpine and riddelliine were assumed to enter the body from the gastrointestinal tract lumen following first order uptake. The in vivo oral absorption rate constant (Ka) of lasiocarpine and riddelliine in human were assumed to be the same as previously determined (Chen et al. 2018). The Ka obtained for lasiocarpine was $1.55 / \mathrm{h}$ and for riddelliine the value amounted to $0.72 / \mathrm{h}$. Model equations were coded (supplementary materials 3) and numerically integrated in Berkeley Madonna 8.0.1 (Macey and Oster, UC Berkeley, CA, USA) using the Rosenbrock's algorithm for stiff systems. 


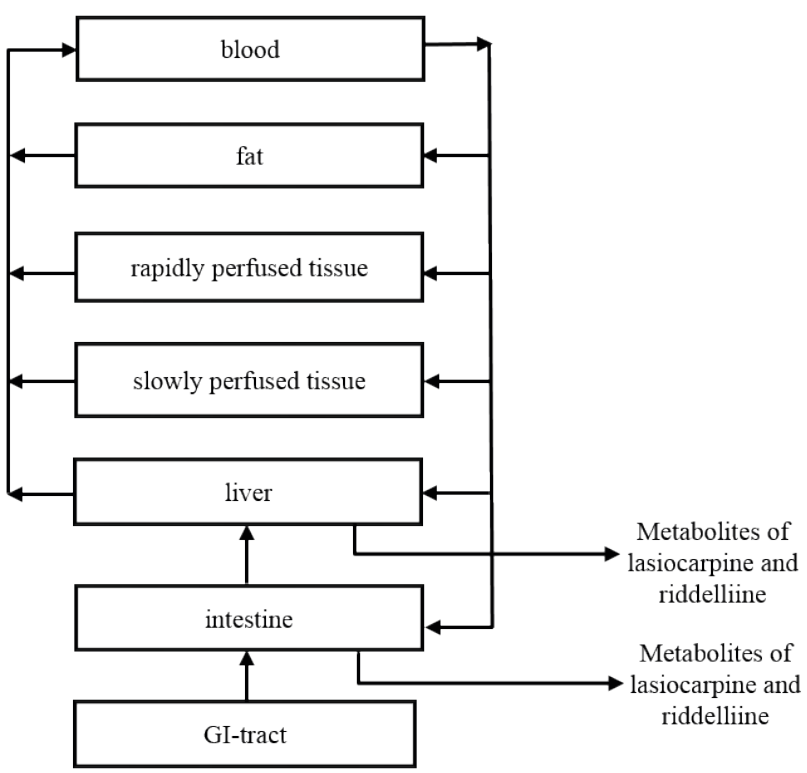

Fig. 2 Schematic diagram of the PBK models for lasiocarpine and riddelliine in human.

\section{Sensitivity analysis}

A sensitivity analysis was performed to identify which parameters have the largest influence on the model predictions. Normalized sensitivity coefficients (SC) were determined using the following equation (3) for the prediction of maximum liver blood concentration $\left(\mathrm{C}_{\max }\right)$ of lasiocarpine and riddelliine:

(3) $\mathrm{SC}=\left(\mathrm{C}^{\prime}-\mathrm{C}\right) /\left(\mathrm{P}^{\prime}-\mathrm{P}\right) \times(\mathrm{P} / \mathrm{C})$

Where $\mathrm{C}$ is the initial value of the model output ( $\mathrm{C}_{\max }$ in liver blood); $\mathrm{C}^{\prime}$ is the modified model output resulting from a $5 \%$ increase of the parameter value; $\mathrm{P}$ is the initial parameter value; $\mathrm{P}^{\prime}$ is the modified parameter value (Evans and Andersen, 2000). A $5 \%$ increase in parameter values was chosen to analyze the effect of a change in parameter values on $\mathrm{C}_{\max }$ of lasiocarpine and riddelliine at a dose of 8 $\mathrm{ng} / \mathrm{kg}$ bw/day and $3 \mathrm{mg} / \mathrm{kg}$ bw/day for 24 hours exposure, representing respectively an average daily intake of PAs via consumption of herbal tea (Bfr, 2013) and a dose level known to cause liver toxicity in humans (Culvenor, 1983). Each parameter was analyzed individually while other parameters were kept at their initial value.

\section{Translation of the in vitro concentration-response curves to in vivo dose-response curves}

The in vitro concentration-response curves on acute cytotoxicity were translated to in vivo doseresponse curves on hepatotoxicity of lasiocarpine and riddelliine by PBK modelling-based reverse dosimetry. For this translation, each concentration applied in the cytotoxicity experiments was 


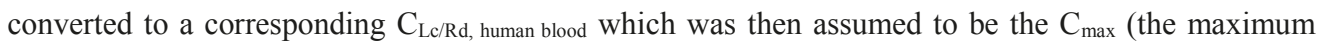
concentration) of lasiocarpine and riddelliine in the liver blood and used as input in the PBK model to determine the corresponding oral dose which would result in acute hepatotoxicity. By performing this exercise for each concentration used in the in vitro cytotoxicity assay, the concentration-response curve was translated to an in vivo dose-response curve.

The in vitro concentration-response curve on cytotoxicity of lasiocarpine and riddelliine for the average Chinese was obtained based on in vitro concentration-response curves on cytotoxicity derived from mixed gender human (Caucasian) liver hepatocytes, correcting the curve for the differences in bioactivation between Chinese and Caucasians as explained above. This was done because mixed gender Chinese liver hepatocytes were not available. To this end each concentration in the cytotoxicity experiment was first multiplied by the fold difference in the peak area of the major glutathione conjugate, 7-GS-DHP formed in liver microsomal incubations in the presence of GSH for Chinese and Caucasian samples. Subsequently, the corrected in vitro concentration-response curves for Chinese was translated to the in vivo dose-response curves based on the same approach as described above for Caucasians using the PBK model for the average Chinese.

\section{BMD analysis of predicted in vivo dose-response data}

BMD modelling was applied on the predicted in vivo dose-response curves to derive the $\mathrm{BMDL}_{5}$ $\mathrm{BMDU}_{5}$ (lower-upper $90 \%$ confidence interval of the benchmark dose that gives a $5 \%$ response) for hepatotoxicity by the PROAST software version 38.9 developed by RIVM (the Dutch National Institute for Public Health and the Environment) (Slob, 2002). This BMD modelling was performed using Exponential and Hill models for continuous data. Models were selected if they passed the goodness-of-fit test at $\mathrm{P}=0.05$ and the ratio of the $\mathrm{BMD}_{5}$ and $\mathrm{BMDL}_{5}$ was not more than 3 -fold (EPA, 2017).

\section{Resultes}

\section{In vitro cytotoxicity}

Figure 3 shows the concentration-response curves for the cytotoxicity of lasiocarpine and riddelliine in HepaRG cells and human pooled hepatocytes from Caucasian donors. Table 1 shows the $\mathrm{IC}_{50}$ values derived from these concentration-response curves. $\mathrm{The}^{\mathrm{IC}_{50}}$ for the cytotoxicity of lasiocarpine and riddelliine towards rat hepatocytes reported in the previous study of Chen et al. (2018) are also shown in Table 1 for comparison (Chen et al., 2018). The results show that rat hepatocytes are more sensitive to lasiocarpine and riddelliine than the HepaRG and human hepatocytes, with the $\mathrm{IC}_{50}$ of lasiocarpine in rat hepatocytes being 20- and 2-fold lower than the $\mathrm{IC}_{50}$ in HepaRG and human hepatocytes, respectively and the $\mathrm{IC}_{50}$ of riddelliine in rat hepatocytes being 22- and 7-fold lower than the $\mathrm{IC}_{50}$ for riddelliine in HepaRG and human hepatocytes, respectively. Therefore, the sensitivity towards the toxicity of lasiocarpine and riddelliine decreases in the order: rat hepatocytes > human hepatocytes > 
$>$ HepaRG cells. In the different cell models the cytotoxicity of lasiocarpine and riddelliine is comparable. For HepaRG cells the $\mathrm{IC}_{50}$ of lasiocarpine is 1.7 -fold higher than that of riddelliine while for human hepatocytes, the $\mathrm{IC}_{50}$ of lasiocarpine is 2.1-fold lower than that of riddelliine. Chen et al. (2018) already reported that the $\mathrm{IC}_{50}$ of lasiocarpine was 1.8 -fold higher than that of riddelliine in rat hepatocytes (Chen et al., 2018).

Given that Chinese human liver hepatocytes are not available, the cytotoxicity of lasiocarpine and riddelliine towards Chinese liver hepatocytes was modelled based on the concentration-response curves obtained with mixed gender Caucasian liver hepatocytes, making a correction for the relative difference in bioactivation as determined in in vitro incubations. Figure 3 also shows the in vitro concentration-response curves of lasiocarpine and riddelliine for the Chinese population obtained in this way. From figure 3 , the $\mathrm{IC}_{50}$ of lasiocarpine and riddelliine were derived for the average Chinese amounting to $126 \pm 39 \mu \mathrm{M}$ and $380 \pm 67 \mu \mathrm{M}$, respectively. Comparing these $\mathrm{IC}_{50}$ values to the values obtained for the average Caucasian (Table 1), reveals that the $\mathrm{IC}_{50}$ values of lasiocarpine and riddelliine for the Chinese is estimated to be 6.0-fold and 8.6-fold higher, respectively. The cytotoxicity data are presented in the supplementary materials 4 .

Table $1 \mathrm{IC}_{50}$ values for the cytotoxicity of lasiocarpine and riddelliine in different cell models

\begin{tabular}{lll}
\hline Cell line & $\mathrm{IC}_{50}(\mu \mathrm{M}) \pm \mathrm{SD}$ & Studies \\
\hline Lasiocarpine & & \\
HepaRG cells & $215 \pm 47$ & Present study \\
Human (Caucasian) hepatocytes & $21 \pm 7$ & Present study \\
Corrected human (Chinese) hepatocytes & $126 \pm 39$ & Present study \\
Rat hepatocytes & $11 \pm 2$ & Chen et al. (2018) \\
Riddelliine & & \\
HepaRG cells & $130 \pm 30$ & Present study \\
Human (Caucasian) hepatocytes & $44 \pm 9$ & Present study \\
Corrected human (Chinese) hepatocytes & $380 \pm 67$ & Present study \\
Rat hepatocytes & $6 \pm 1$ & Chen et al. (2018) \\
\hline
\end{tabular}




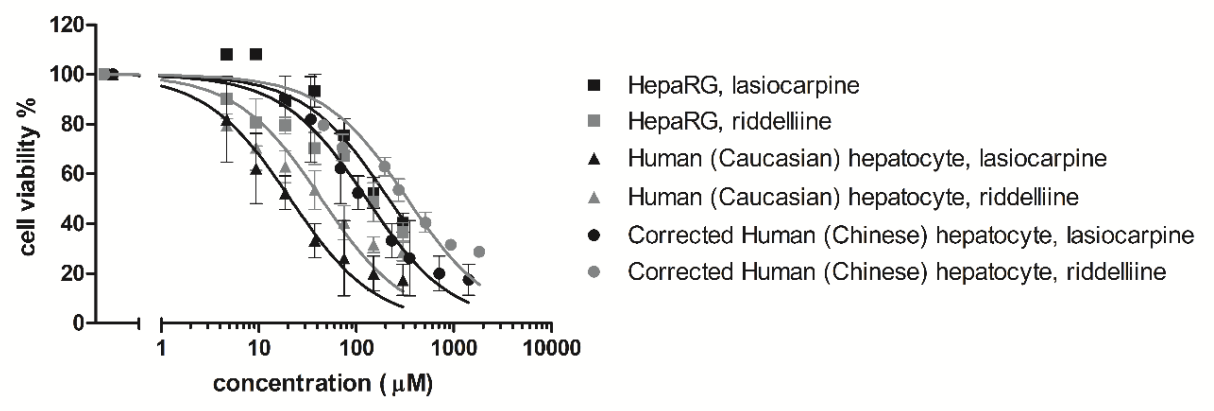

Fig. 3 Concentration-response curves for the cytotoxicity of lasiocarpine (black line) and riddelliine (grey line) in HepaRG cells (squares), human (Caucasian) hepatocytes (triangles) and corrected human (Chinese) hepatocytes (circles) upon 24 hours exposure (mean values $\pm \mathrm{SD}$ ).

\section{Microsomal conversion of lasiocarpine and riddelliine by Chinese and Caucasian tissue fractions}

Upon incubation of increasing concentrations of lasiocarpine and riddelliine with Chinese and Caucasian tissue fractions and NADPH, the extent of substrate depletion was quantified by UPLCPDA analysis. Table 2 displays the apparent $\mathrm{V}_{\max }$ and $\mathrm{K}_{\mathrm{m}}$ values obtained from the plots as shown in Figure 4 and the catalytic efficiency, calculated as $V_{\max } / K_{m}$. Conversion of lasiocarpine and riddelliine was detected in incubations with liver and intestinal microsomes. Incubations of lasiocarpine and riddelliine with lung and kidney microsomes of Caucasians did not result in detectable metabolic conversion. Lung and kidney microsomes from Chinese individuals were not available, but it was assumed that they would also not cause metabolic conversion of lasiocarpine and riddelliine. Liver was the major organ for lasiocarpine and riddelliine metabolism, as reflected by the fact that the catalytic efficiency for the depletion of lasiocarpine or riddelliine in incubations with pooled liver microsomes from Caucasian individuals is around 15-fold higher than the catalytic efficiency derived from incubations with intestinal microsomes. Comparison of the kinetic constants for lasiocarpine and riddelliine reveals that for depletion of lasiocarpine by Chinese liver fractions, the $\mathrm{V}_{\max }$ is 15.3 -fold higher and the $\mathrm{K}_{\mathrm{m}}$ is 3.7 -fold lower than for riddelliine, resulting in a 54.8 -fold higher catalytic efficiency for lasiocarpine metabolism. For Caucasian liver samples, the $\mathrm{V}_{\max }$ and $\mathrm{K}_{\mathrm{m}}$ for depletion of lasiocarpine are 2.6-fold higher and 10.5-fold lower, respectively, than those of riddelliine, resulting in a 27.1-fold higher catalytic efficiency for depletion of lasiocarpine. In the case of the intestine, the apparent $\mathrm{V}_{\max }$ and $\mathrm{K}_{\mathrm{m}}$ for the depletion of lasiocarpine in incubations with small intestinal microsomes from Caucasians are 5.0-fold higher and 5.3-fold lower, respectively, than those of riddelliine. The catalytic efficiency for depletion of lasiocarpine in these intestinal incubations is 25 -fold higher than for depletion of riddelliine.

Comparison of the kinetic data for the depletion of lasiocarpine and riddelliine by liver fractions from 
the Chinese and Caucasians reveals that the catalytic efficiency for both compounds by the Chinese liver samples is 2.3 to 4.6 -fold lower, which is mainly due to a 2.0 -fold higher $\mathrm{K}_{\mathrm{m}}$ for depletion of lasiocarpine and 6.7-fold lower $\mathrm{V}_{\max }$ for depletion of riddelliine in Chinese liver. Comparison of the kinetic data for formation of 7-GS-DHP of both compounds by Chinese and Caucasian liver fractions, reveals that over the substrate concentration range used in incubations with Chinese liver microsomes bioactivation of lasiocarpine and riddelliine is 7.5-fold and 8.1-fold lower, respectively. $\mathrm{V}_{\max }$ values in these incubations were not quantified because for correction of the in vitro curves only the relative ratio in bioactivation were required. The curves of the concentration-dependent rate of formation of $7-$ GS-DHP of both compounds, expressed in peak area $/ \mathrm{min} / \mathrm{mg}$ liver protein and the ratio of the peak areas of 7-GS-DHP formed in incubations with Chinese and Caucasian liver microsomes at each substrate concentrationare presented in the supplementary materials 5. Since the 7-GS-DHP metabolite formed from lasiocarpine and riddelliine is the same, also bioactivation of the two compounds can be compared based on peak areas.

Table 2 Kinetic constants for depletion of lasiocarpine and riddelliine by Chinese and Caucasian tissue fractions

\begin{tabular}{|c|c|c|c|c|c|c|}
\hline \multirow[b]{2}{*}{ Organ } & \multicolumn{3}{|c|}{ Chinese } & \multicolumn{3}{|c|}{ Caucasian } \\
\hline & $\begin{array}{c}\mathrm{V}_{\max }{ }^{\mathrm{a}} \\
(\mathrm{nmol} / \mathrm{min} / \mathrm{mg} \\
\text { microsomal } \\
\text { protein) }\end{array}$ & $\begin{array}{l}\mathrm{K}_{\mathrm{m}}{ }^{\mathrm{a}} \\
(\mu \mathrm{M})\end{array}$ & $\begin{array}{c}\text { Catalytic } \\
\text { efficiency }{ }^{b} \\
(\mu \mathrm{L} / \mathrm{min} / \mathrm{mg} \\
\text { microsomal } \\
\text { protein) }\end{array}$ & $\begin{array}{c}\mathrm{V}_{\max }{ }^{\mathrm{a}} \\
\text { (nmol/min/mg } \\
\text { microsomal } \\
\text { protein) }\end{array}$ & $\begin{array}{l}\mathrm{K}_{\mathrm{m}}{ }^{\mathrm{a}} \\
(\mu \mathrm{M})\end{array}$ & $\begin{array}{c}\text { Catalytic } \\
\text { efficiency } \\
(\mu \mathrm{L} / \mathrm{min} / \mathrm{mg} \\
\text { microsomal } \\
\text { protein) }\end{array}$ \\
\hline \multicolumn{7}{|c|}{ Lasiocarpine } \\
\hline Liver & $4.6 \pm 0.9$ & $52.5 \pm 23.2$ & 87.8 & $5.1 \pm 0.9$ & $25.8 \pm 13.0$ & 197.7 \\
\hline Intestine & n.a. ${ }^{c}$ & n.a. ${ }^{c}$ & - & $0.9 \pm 0.2$ & $72.1 \pm 37.9$ & 12.5 \\
\hline Lung & n.a. ${ }^{c}$ & n.a. ${ }^{c}$ & - & n.d. ${ }^{d}$ & n.d. ${ }^{d}$ & - \\
\hline Kidney & n.a. ${ }^{c}$ & n.a. ${ }^{c}$ & - & n.d. ${ }^{d}$ & n.d. ${ }^{\mathrm{d}}$ & - \\
\hline \multicolumn{7}{|l|}{ Riddelliine } \\
\hline Liver & $0.3 \pm 0.05$ & $195.5 \pm 61.0$ & 1.6 & $2.0 \pm 0.5$ & $274.1 \pm 150.7$ & 7.3 \\
\hline Intestine & n.a. ${ }^{c}$ & n.a. ${ }^{c}$ & - & $0.2 \pm 0.1$ & $380.4 \pm 285.3$ & 0.5 \\
\hline Lung & n.a. ${ }^{c}$ & n.a. ${ }^{c}$ & - & n.d. ${ }^{d}$ & n.d. ${ }^{d}$ & - \\
\hline Kidney & n.a. ${ }^{\mathrm{c}}$ & n.a. ${ }^{c}$ & - & n.d. ${ }^{d}$ & n.d. ${ }^{d}$ & - \\
\hline
\end{tabular}

\footnotetext{
${ }^{\text {a }}$ Average \pm SD of three independent experiments

${ }^{\mathrm{b}} \mathrm{V}_{\max } / \mathrm{K}_{\mathrm{m}} \times 1000$

${ }^{\mathrm{c}}$ Not available

${ }^{\mathrm{d}}$ Not detected
} 


\section{Lasiocarpine}

$a$

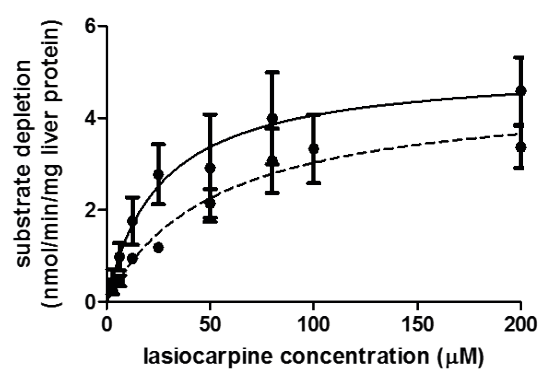

b

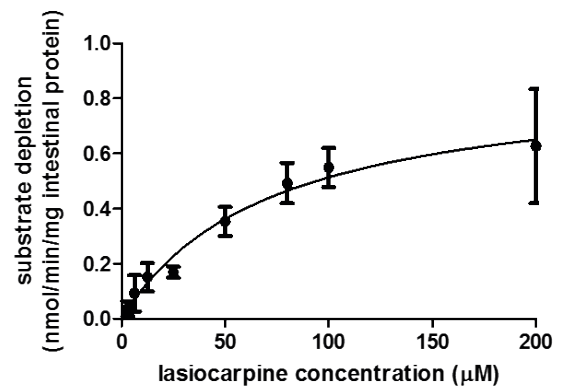

Riddelliine
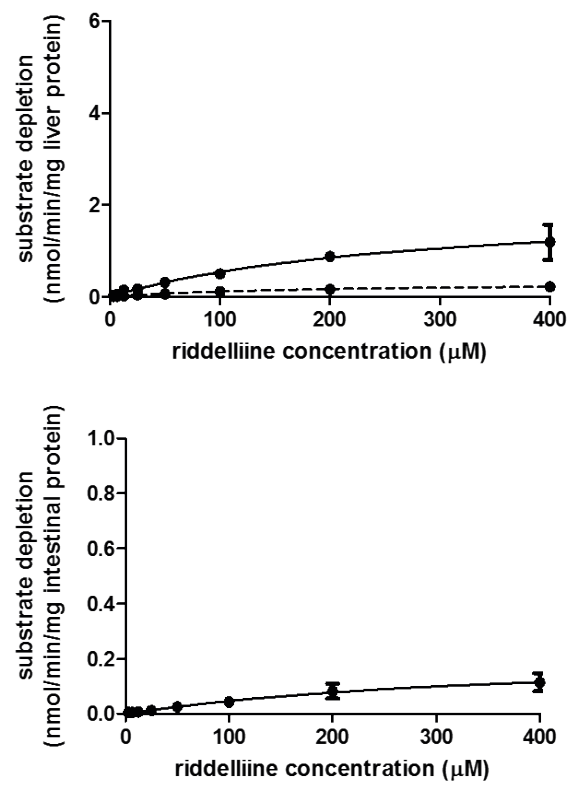

Fig. 4 Concentration-dependent rate of depletion of lasiocarpine and riddelliine in incubations with Chinese (dotted line) and Caucasian (solid line) liver microsomes (a) and Caucasian intestinal microsomes (b) in the presence of the cofactor NADPH. Data points represent mean values $\pm \mathrm{SD}$ of three individual experiments.

\section{Comparison of the scaled kinetic constants for lasiocarpine and riddelliine by human}

\section{and rat tissue fractions}

In a subsequent step the kinetic constants presented in table 2 were scaled to human tissues. To this end, the in vitro $\mathrm{V}_{\max }$ values expressed as $\mathrm{nmol} / \mathrm{min} / \mathrm{mg}$ microsomal protein (Table 2) were scaled to $\mu \mathrm{mol} / \mathrm{h} / \mathrm{kg}$ bw using the microsomal protein yields of $35 \mathrm{mg} /(\mathrm{g}$ liver) or $20.6 \mathrm{mg} /(\mathrm{g}$ small intestine) as scaling factors as described previously (Cubitt et al., 2009; Medinsky et al., 1994), and bodyweight and tissue fractions of human (Chinese and Caucasian) and rat (Chen et al., 2018). This scaling allows the comparison of the kinetic constants obtained from tissue fractions of different species and from different organs. Table 3 presents a summary of the kinetic parameters for lasiocarpine and riddelliine by human (Chinese and Caucasian) tissue fractions thus obtained and the comparable kinetic parameter values previously reported for rat tissue fractions (Chen et al., 2018).

Comparison of the kinetic constants between human (Chinese and Caucasians) and rat shows that liver is the major organ for lasiocarpine and riddelliine metabolism. Compared to the human (Caucasian), the contribution of intestinal metabolism for both compounds is higher in the rat. The catalytic efficiency for the depletion of lasiocarpine is generally 3.2-fold lower in human liver (Chinese and Caucasian) and 6-fold lower in human intestine (Caucasian) than in rat. The overall catalytic 
efficiency for depletion of lasiocarpine in human (Caucasian) is 1.9-fold lower than in rat. The catalytic efficiency for the depletion of riddelliine is generally 16.8-fold lower in human liver (Chinese and Caucasian) and 1.2-fold lower in small intestine (Caucasian) compared to those values in rat tissues. The overall catalytic efficiency for depletion of riddelliine in human (Caucasian) is 5.0-fold lower. Those generally lower values of catalytic efficiency for depletion of lasiocarpine and riddelliine in human liver and small intestine compared to the rat were mainly due to relatively higher $\mathrm{K}_{\mathrm{m}}$ values for depletion of both compounds in human.

Table 3 Scaled kinetic constants for depletion of lasiocarpine and riddelliine by human (Chinese and Caucasian) and rat tissue fractions

\begin{tabular}{|c|c|c|c|c|c|c|}
\hline \multirow{4}{*}{ Organ } & \multicolumn{3}{|c|}{ Human (Chinese/Caucasian) } & \multicolumn{3}{|c|}{ Rat } \\
\hline & Scaled $V_{\max }{ }^{a}$ & $\mathrm{~K}_{\mathrm{m}}$ & Scaled catalytic & Scaled $V_{\max }{ }^{a, c}$ & $\mathrm{~K}_{\mathrm{m}}^{\mathrm{a}, \mathrm{c}}$ & Scaled catalytic \\
\hline & $(\mu \mathrm{mol} / \mathrm{h} / \mathrm{kg} \mathrm{bw})$ & $(\mu \mathrm{M})$ & efficiency $^{\mathrm{b}}$ & $(\mu \mathrm{mol} / \mathrm{h} / \mathrm{kg} \mathrm{bw})$ & $(\mu \mathrm{M})$ & efficiency ${ }^{b, c}$ \\
\hline & & & $(\mathrm{L} / \mathrm{h} / \mathrm{kg} \mathrm{bw})$ & & & (L/h/kg bw) \\
\hline \multicolumn{7}{|l|}{ Lasiocarpine } \\
\hline Liver & $222.2^{\mathrm{e} / 278.2^{\mathrm{f}}}$ & $52.5^{\mathrm{e}} / 25.8^{\mathrm{f}}$ & $4.2^{\mathrm{e}} / 10.8^{\mathrm{f}}$ & 377.4 & 19.5 & 19.4 \\
\hline Intestine & n. $\mathrm{a}^{\mathrm{d}, \mathrm{e}} / 9.9^{\mathrm{f}}$ & n. $\mathrm{a}^{\mathrm{d}, \mathrm{e}} / 72.1^{\mathrm{f}}$ & n.a ${ }^{\mathrm{d}, \mathrm{e}} / 0.2^{\mathrm{f}}$ & 29.4 & 23.4 & 1.3 \\
\hline \multicolumn{7}{|l|}{ Riddelliine } \\
\hline Liver & $14.5^{\mathrm{e} / 109.2^{\mathrm{f}}}$ & $195.5^{\mathrm{e} / 274.1^{\mathrm{f}}}$ & $0.07^{\mathrm{e}} / 0.4^{\mathrm{f}}$ & 149.6 & 75.7 & 2.0 \\
\hline Intestine & n. $\mathrm{a}^{\mathrm{d}, \mathrm{e}} / 1.8^{\mathrm{f}}$ & n.a ${ }^{\mathrm{d}, \mathrm{e}} / 380.4^{\mathrm{f}}$ & n.a ${ }^{\mathrm{d}, \mathrm{e}} / 0.005^{\mathrm{f}}$ & 1.4 & 221.0 & 0.006 \\
\hline
\end{tabular}

${ }^{\text {a }}$ Scaled $\mathrm{V}_{\max }$ calculated from the in vitro $\mathrm{V}_{\max }$ using the scaling factor of microsome protein yields of $35 \mathrm{mg} /(\mathrm{g}$

liver) or $20.6 \mathrm{mg} /(\mathrm{g}$ small intestine), bodyweight and tissue fractions of human (Chinese and Caucasian) and rat

${ }^{\mathrm{b}}$ Scaled catalytic efficiency (scaled $\mathrm{V}_{\max } / \mathrm{K}_{\mathrm{m}}$ )

${ }^{\mathrm{c}}$ Chen et al. 2018

${ }^{\mathrm{d}}$ Not available

${ }^{\text {e }}$ Scaled kinetic constants by Chinese tissue fractions

${ }^{\mathrm{f}}$ Scaled kinetic constants by Caucasian tissue fractions

\section{Unbound fraction in human blood as determined in the RED assay}

The unbound fraction for lasiocarpine in human plasma was determined to be 0.43 . For as yet unknown reasons, and in contrast to lasiocarpine, the unbound faction $\left(f_{u b}\right)$ of riddelliine could not be detected after incubation in the RED device, even not when the concentration was increased from 5 to $50 \mu \mathrm{M}$. Previously, Chen et al. (2018) defined $\mathrm{f}_{\mathrm{ub}}$ values for lasiocarpine and riddelliine by rat serum using the same method as provided in above, and reported that the $f_{u b}$ values of lasiocarpine and riddelliine in rat plasma were comparable, amounting to 0.64 for lasiocarpine and to 0.66 for riddelliine. Therefore, in the present study the $f_{u b}$ of riddelliine in human plasma was assumed to be the same as the $f_{u b}$ value of lasiocarpine in human plasma amounting to 0.43 . 


\section{PBK model prediction and evaluation}

To evaluate the performance of the human PBK models, ideally the model outcomes should be compared to available human data. Since such human in vivo data on the kinetics of lasiocarpine and riddelliine are not available, model evaluation is mainly based on evaluation of the same model in our previous study where we developed and evaluated the model for riddellliine and lasiocarpine in rat and mouse (Chen et al., 2018). Chen et al. (2018) described the performance of the rat and mouse PBK model of riddelliine by comparing the predicted $\mathrm{C}_{\max }$ of riddelliine in rat and mouse blood to the observed in vivo data reported in the literature (Chen et al., 2018). Data revealed that the predicted levels of $\mathrm{C}_{\max }$ of riddelliine in rat and mouse blood and the $\mathrm{C}_{\max }$ values observed in these species in the in vivo studies matched adequately (Chen et al., 2018). An additional evaluation of the model emerged from comparison of the PBK modelling-based predicted $\mathrm{BMDL}_{5}-\mathrm{BMDU}_{5}$ values derived from the predicted in vivo dose-response curve for liver toxicity of lasiocarpine in rats. The results fell well within the range of PoDs obtained from actual in vivo studies on acute liver toxicity of lasiocarpine in rats, indicating that the PoDs derived from the combined in vitro and in silico PBK modelling approach provided a good approximation of in vivo acute liver toxicity of lasiocarpine (Chen et al., 2018). Based on these results for the rat model, the developed PBK model for lasiocarpine or riddelliine are considered an adequate first approximation to describe the in vivo situation for human. An additional approach to describe the performance of the human PBK model for lasiocarpine and riddelliine for the human populations, especially for the difference between the Chinese and the Caucasian populations, was done based on the approach described previously (Ning et al., 2017). In this approach a comparison is made between the predicted inter-ethnic differences for the dosedependent concentration of lasiocarpine and riddelliine in the liver blood and the observed inter-ethnic differences in hepatic metabolising enzymes that mainly catalyse the depletion of lasiocarpine or riddelliine. CYP 3A4 predominately catalyses the depletion of lasiocarpine and riddelliine in human liver (Fu et al., 2004; Miranda et al., 1991; Wiedenfeld and Edgar, 2011). Figure 5 shows that at similar dose levels the predicted $\mathrm{C}_{\max }$ of lasiocarpine and riddelliine in the liver blood from average Chinese subjects is generally 2- to 3-fold higher than in the liver from average Caucasian subjects. This is mainly due to the fact that the catalytic efficiency for depletion of lasiocarpine and riddelliine by Chinese liver microsomes is 2- to 4-fold lower compared to Caucasian liver mirosomes. The prediction of higher $\mathrm{C}_{\max }$ values for lasiocarpine and riddelliine for Chinese subjects is in line with the reported data for the $C_{\max }$ and AUC of alprazolam, the substrate for CYP $3 A 4 / 5$, for which the $C_{\max }$ and AUC for Asian subjects (ten Chinese, three Filipino, and one Japanese) were 1.2- and 1.3-fold, respectively, higher than for Caucasian subjects $(\mathrm{n}=14)$ (Lin et al., 1988). Furthermore, in a metaanalysis Barter et al. (2013) showed that the plasma clearance of the CYP 3A4/5 substrate midazolam following an oral dosing was 2.4-fold higher in Caucasian than in Chinese subjects. 
For the inter-species comparison, the predicted $\mathrm{C}_{\max }$ of lasiocarpine and riddelliine in the human (Chinese and Caucasian) liver blood is generally 2- to 5-fold higher than those in the rat liver blood as shown in figure 5. This is because the catalytic efficiency for depletion of both compounds in human (Caucasian) is 2- to 5-fold lower compared to rat. Altogether, the PBK models obtained in the present study were considered adequate for further prediction of the inter-species and inter-ethnic differences in hepatotoxicity of lasiocarpine and riddelliine.
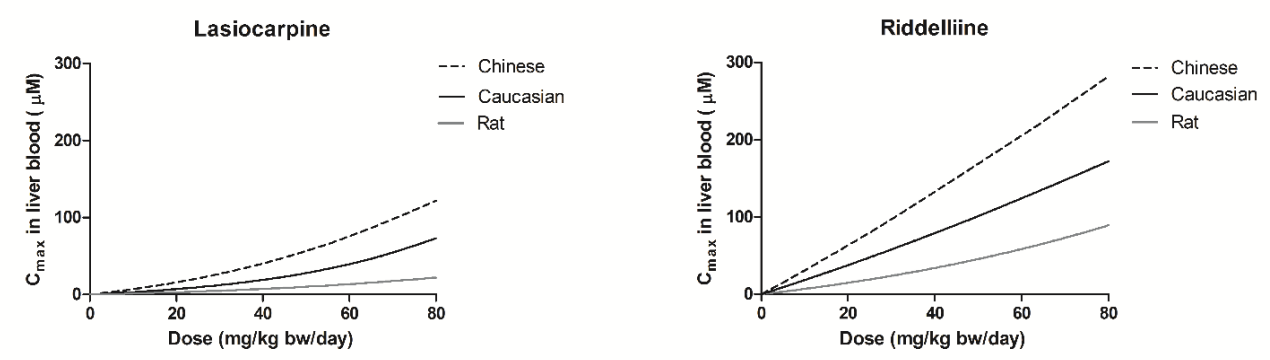

Fig. 5 PBK modelling-based predictions for the dose-dependent $\mathrm{C}_{\max }$ of lasiocarpine and riddelliine in liver blood in Chinese (black dotted line), Caucasian (black solid line) and rat (grey line).

\section{Sensitivity analysis}

The normalized sensitivity coefficients of the model parameters for prediction of the $\mathrm{C}_{\max }$ of lasiocarpine and riddelliine in liver blood of Chinese and Caucasian was analyzed. The sensitivity coefficients were calculated at a dose of $8 \mathrm{ng} / \mathrm{kg}$ bw and $3 \mathrm{mg} / \mathrm{kg}$ bw lasiocarpine and riddelliine and all model parameters with sensitivity coefficients higher than $|0.1|$ are shown in the supplementary materials 6. The results obtained reveal that for both Chinese and Caucasian subjects at a dose of 8 $\mathrm{ng} / \mathrm{kg}$ bw lasiocarpine the predicted $\mathrm{C}_{\max }$ in liver blood in the PBK model is most sensitive to liver related parameters and the absorption rate constant $(\mathrm{Ka})$ for uptake from the GI tract. At $3 \mathrm{mg} / \mathrm{kg}$ bw lasiocarpine, the results of the sensitivity analysis of both ethnic groups are similar to the situation at 8 $\mathrm{ng} / \mathrm{kg}$ bw lasiocarpine. The results for the sensitivity analysis of the PBK models for riddelliine shown in Figure S6 reveal similar results showing that $\mathrm{Ka}$ and liver related parameters have the highest influence on the predicted $\mathrm{C}_{\max }$ of riddelliine in the liver blood in both ethnic groups. At $8 \mathrm{ng} / \mathrm{kg}$ bw riddelliine the results of the sensitivity analysis of both ethnic groups are similar to the analysis at 3 $\mathrm{mg} / \mathrm{kg}$ bw. For both compounds, Ka and liver related parameters have less influence on the predicted $\mathrm{C}_{\max }$ in the liver blood in average Chinese compared to average Caucasian.

\section{Predicted inter-ethnic differences in hepatotoxicity of lasiocarpine and riddelliine}

Figure 6 shows the predicted in vivo dose-response curves for liver toxicity of lasiocarpine and riddelliine in Chinese and Caucasian subjects obtained by translation of the in vitro concentrationresponse curves obtained from human hepatocytes (Figure 3) by reverse dosimetry using the respective PBK models. The response \% indicated at the y-axis of the in vivo dose-response curve was 
calculated based on the equation (4): response $\%=100 \%$ (solvent control) - cell viability $\%$ in the in vitro concentration response curve.

Table 4 presents the $\mathrm{BMDL}_{5}-\mathrm{BMDU}_{5}$ values derived from the predicted dose-response curves as shown in figure 6 for Chinese and Caucasian subjects. The values of the $\mathrm{BMD}_{5}$ (benchmark dose corresponding to $5 \%$ extra risk) were also presented to enable calculation of the ratio between the $\mathrm{BMD}_{5}$ and $\mathrm{BMDL}_{5} / \mathrm{BMDU}_{5}$ showing they are generally lower than 3, which was one of the criteria for acceptance of the model fit. Detailed information on the BMD analysis can be found in the supplementary materials 7 .

Interestingly, the PBK modelling-based predictions for in vivo human toxicity of riddelliine could be evaluated using available human data, supporting further evaluation of the PBK models and the PBK modelling-based reverse dosimetry approach. The study of Culvenor (1983) estimated that in human subjects a dose of $1.4-3 \mathrm{mg} / \mathrm{kg}$ bw/day and $0.7-1.5 \mathrm{mg} / \mathrm{kg}$ bw/day of riddelliine may lead to liver necrosis (exposure of a boy for shorter than 2 weeks) and liver cirrhosis (exposure of a girl for 2 weeks), respectively (Culvenor, 1983). The $\mathrm{BMD}_{5}$ values for Caucasian and Chinese subjects predicted in the present study are comparable since they amount to 0.5 and $2.6 \mathrm{mg} / \mathrm{kg}$ bw/day, respectively. For the inter-ethnic comparison, the predicted $\mathrm{BMDL}_{5}$ of lasiocarpine was observed to be 2.0-fold higher for Chinese than for Caucasian subjects and for riddelliine the predicted $\mathrm{BMDL}_{5}$ value was 5.0-fold higher for Chinese than for Caucasian subjects, indicating Chinese may be less sensitive to the liver toxicity of these PAs than the Caucasian population. This difference can be mainly ascribed to the lower relative level of bioactivation resulting in lower toxicity at similar dose levels in spite of the slower metabolic clearance.

Table 4 Predicted $\mathrm{BMD}_{5}$ and $\mathrm{BMDL}_{5}-\mathrm{BMDU}_{5}$ values derived from dose-response curves predicted using PBK modelling-based reverse dosimetry of in vitro cytotoxicity data with human hepatocytes

\begin{tabular}{lll}
\hline \multirow{2}{*}{ Compounds } & \multicolumn{2}{l}{ Predicted BMDL $-\mathrm{BMDU}_{5}\left(\mathrm{BMD}_{5}\right)(\mathrm{mg} / \mathrm{kg}$ bw/day $)$} \\
\cline { 2 - 3 } & Chinese & Caucasian \\
\hline Lasiocarpine & $14.7-41.2(26.0)$ & $7.4-23.7(14.1)$ \\
Riddelliine & $1.0-5.9(2.6)$ & $0.2-1.2(0.5)$ \\
\hline
\end{tabular}


Lasiocarpine

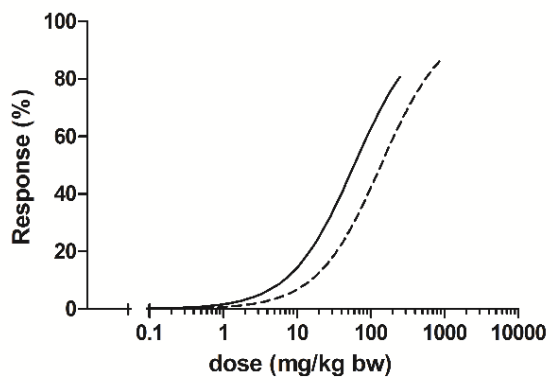

Riddelliine

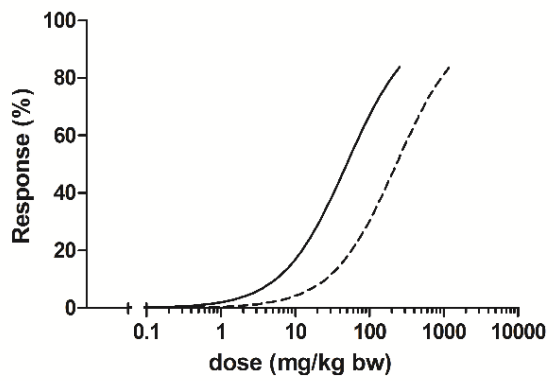

Fig. 6 Predicted dose-response curves for acute liver toxicity of lasiocarpine and riddelliine in average Chinese (black dotted line) and average Caucasians (black solid line) as obtained by translation of cytotoxicity concentration-response data obtained with human hepatocytes using PBK modelling-based reverse dosimetry

\section{Predicted species differences in hepatotoxicity of lasiocarpine and riddelliine}

To obtain insight in possible species differences in sensitivity toward liver toxicity of the PAs, the $\mathrm{BMDL}_{5}-\mathrm{BMDU}_{5}$ values of lasiocarpine and riddelliine for acute liver toxicity for humans (Chinese and Caucasians) (Table 4) defined in the present study were compared to the same PoDs defined previously for rats using in vitro toxicity data in rat hepatocytes and PBK models for rats (Chen et al. 2018) (see Figure 7). The $\mathrm{BMDL}_{5}-\mathrm{BMDU}_{5}$ values derived from the predicted dose-response curves for lasiocarpine for humans amounting to $11.1-32.3 \mathrm{mg} / \mathrm{kg}$ bw (average PoDs of Chinese and Caucasians) are up to 2.0-fold lower than the predicted $\mathrm{BMDL}_{5}-\mathrm{BMDU}_{5}$ values of Chen et al. (2018) for lasiocarpine for rat which amounted to $23.0-34.4 \mathrm{mg} / \mathrm{kg}$ bw/day. For riddelliine, the predicted $\mathrm{BMDL}_{5}-\mathrm{BMDU}_{5}$ values for humans amounting to $0.6-3.6 \mathrm{mg} / \mathrm{kg}$ bw (average PoDs of Chinese and Caucasians) are up to 8.2 -fold lower than the $\mathrm{BMDL}_{5}-\mathrm{BMDU}_{5}$ for rat amounting to $4.9-8.4 \mathrm{mg} / \mathrm{kg}$ bw/day. Thus humans seem to be somewhat more sensitive to liver toxicity of these PAs than rats. 


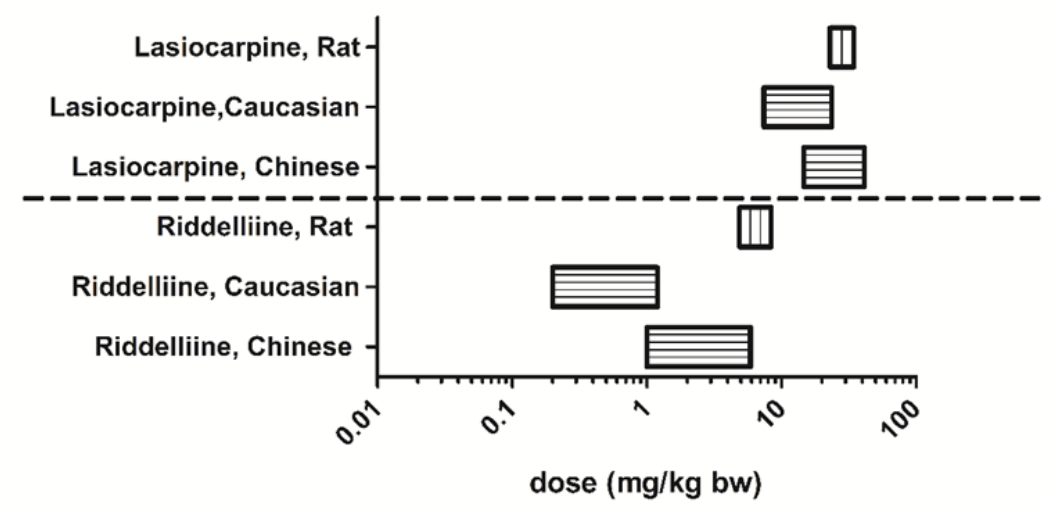

Fig. 7 Predicted BMDL5-BMDU5 values for acute liver toxicity of lasiocarpine (above the dashed line) and riddelliine (below the dashed line) in humans (Caucasians and Chinese) (bars containing horizontal lines) predicted by the PBK modelling-based reversed dosimetry approach using data for human hepatocytes from the present study as compared to the predicted $\mathrm{BMDL}_{5}-\mathrm{BMDU}_{5}$ values for acute liver toxicity of lasiocarpine (above the dashed line) and riddelliine (below the dashed line) in rat (bars containing vertical lines) derived from our previous study using data for rat hepatocytes and a rat PBK model (Chen et al., 2018).

\section{Discussion}

The aim of the present study was to use PBK modelling-based reverse dosimetry to translate human cytotoxicity data of lasiocarpine and riddelliine to in vivo dose-response data in humans (Chinese and Caucasians) and study ethnic human differences. Comparison of the results to a similar study previously performed for rats (Chen et al., 2018) would provide insight in species differences in sensitivity to these two PAs.

The PBK model developed in the present study describes the kinetics of lasiocarpine and riddelliine and not of their metabolites, since we assume that the acute liver toxicity of lasiocarpine and riddelliine can be modelled based on cytotoxicity data of the parent compound provided that the in vitro model used contains the adequate enzymes required for their bioactivation to hepatotoxic metabolites. Especially the dehydropyrrolizidine alkaloids metabolites of PAs are reactive with proteins and DNA, thereby causing toxic effects (Fu et al., 2004). The in vitro cytotoxicity experiment using HepaRG cells and human hepatocytes can be expected to take this bioactivation into account. The HepaRG cell line is a human hepatoma cellular model consisting of a mixture of both hepatocytelike and biliary-like cells (Lambert et al., 2009). After the treatment with DMSO, HepaRG cells differentiate into hepatocyte-like morphology. Kanebratt and Andersson (2008) reported that the expression of P450 in HepaRG cells was generally lower compared with human hepatocytes, except for a higher expression of CYP 3A4 and CYP 7A1. However, the CYP 3A4 activity was still about1.5fold higher in human hepatocytes in 2 out of 3 individuals compared to DMSO treated HepaRG cells 
as determined by measuring the clearance of the CYP 3A4 probe substrate midazolam (Kanebratt and Andersson, 2008). Other studies also showed that P450 activities such as CYP 1A2, CYP 2B6, CYP 2C8, CYP 2C9, CYP 2C19 and CYP 2D6 were generally lower in HepaRG cells than in human hepatocytes, expect for CYP 3A4 showing a generally 1.5-fold higher activity in HepaRG cells (Kvist et al., 2018; Lubberstedt et al., 2011). However, Gerets et al. (2012) reported that CYP 3A4 activity was about 17.0-fold lower in the HepaRG cells than in freshly isolated human hepatocytes from three different donors. This conflicting result may result from inter-individual variability in CYP 3A4 activity and/or from differences in the level of enzyme induction the DMSO treated HepaRG cells. However based on the limited difference in the cytotoxicity of riddelliine and lasiocarpine in primary hepatocytes and the HepaRG cells observed in the present study (Figure 3), we conclude that induction of the CYPs involved in bioactivation of these PAs in the HepRG cells was adequate. Gerets et al. (2012) also reported that cryopreserved primary human hepatocytes showed a 3-fold higher sensitivity for the detection of hepatotoxic compounds compared with HepaRG cells. In the present study, the $\mathrm{IC}_{50}$ value for lasiocarpine and riddelliine derived from HepaRG cells was 10- and 3-fold higher, respectively, than the $\mathrm{IC}_{50}$ derived from studies with human hepatocytes, probably reflecting the lower metabolic capacity of the HepaRG cells for bioactivation of the PAs. Given these differences, prediction of the acute liver toxicity of both PAs was based on the in vitro concentration-response curves obtained with human primary hepatocytes.

Based on the sensitivity analysis, the predicted $\mathrm{C}_{\max }$ values of lasiocarpine and riddelliine in average Chinese and average Caucasians are highly affected by the absorption rate constant (Ka) for uptake from the GI tract and the kinetic constants for metabolic clearance of the parent compounds in the liver. Variability in the kinetic constants for metabolic clearance can result from the individual differences in the expression of CYP 3A4 which is the primary enzyme for metabolizing lasiocarpine and riddelliine in humans (Miranda et al., 1991). The literature reports that the abundance of CYP 3A4 in the liver varies significantly in individuals (Fu et al., 2004), and the inter-individual variation in the level of CYP 3A4 expression can vary up to 100 -fold (Westlind-Johnsson et al., 2003). In addition to the kinetic constants, the Ka value also has a large influence on the model predictions. In the present study, the Ka values of lasiocarpine and riddelliine were $1.55 / \mathrm{h}$ and $0.72 / \mathrm{h}$, respectively as reported by Chen et al. (2018). In the study of Chen et al. (2018), Ka values of both compounds were defined based on $\mathrm{P}_{\text {app }}$ values derived by two methods: Qikprop software (SchrÖdinger, trial version, Germany) and a QSAR method developed by Hou et al. (2004). The $\mathrm{P}_{\text {app }}$ values obtained from these two methods differ only 1.3-fold for lasiocarpine, but 4.5-fold for riddelliine (Chen et al., 2018). The large difference in $\mathrm{P}_{\mathrm{app}}$ values for riddelliine may therefore largely affect PBK model-predicted internal concentrations and related predicted dose levels that cause acute toxicity. Previously, Chen et al. (2018) reported the predicted $\mathrm{BMDL}_{5}-\mathrm{BMDU}_{5}$ for riddelliine in rat amounting to $1.0-2.2 \mathrm{mg} / \mathrm{kg}$ bw/day when using the $\mathrm{Ka}(0.27 / \mathrm{h})$ obtained by Qikprop software and to $3.3-14.6 \mathrm{mg} / \mathrm{kg}$ bw/day when using the 
$\mathrm{Ka}(1.17 / \mathrm{h})$ obtained by the QSAR method of Hou et al. (2004). The PoDs for riddelliine obtained in rats when using the average $\mathrm{Ka}$ amount to $4.9-8.4 \mathrm{mg} / \mathrm{kg}$ bw/day (Chen et al., 2018). This outcome supports the outcome of the sensitivity analysis indicating that the Ka value has a large influence on the model predictions. This leads to the conclusions that further refinement of especially the $\mathrm{P}_{\text {app }}$ and resulting Ka value may improve the models and their predictions. However, given that the predicted $\mathrm{BMD}_{5}$ values for riddelliine in Chinese and Caucasian subjects amounting to 2.6 and $0.5 \mathrm{mg} / \mathrm{kg}$ bw/day, respectively are comparable to the literature reported in vivo data for human subjects amounting to $0.7-3 \mathrm{mg} / \mathrm{kg}$ bw/day (Culvenor, 1983) when using the average Ka values, the use of the average Ka value for riddelliine seems a reasonable strategy. A recent study reported that the pyrrolizidine alkaloid monocrotaline (retronecine-type PA) is a high affinity substrate of the organic cation transporter 1 (OCT1), a transporter mainly expressed in liver and involved in uptake of chemicals from blood into the hepatocytes. This study suggested that active transport mechanisms may be involved in the cellular uptake of pyrrolizidine alkaloids into the liver tissue (Tu et al., 2013). In the present study, we used $\log \mathrm{K}_{\mathrm{ow}}$ to estimate the liver tissue:blood partition coefficients of lasiocarpine and riddelliine. In perfusion-limited PBK models, such as used in our study, an immediate partitioning between plasma and tissue is assumed, which is not dependent on chemical diffusion across cell membranes and/or active uptake processes. Therefore we do not expect to underestimate hepatic concentrations in our models. Given that the sensitivity analysis revealed that the $\mathrm{C}_{\max }$ of lasiocarpine and riddelliine in liver blood were not highly affected by the liver tissue:blood partition coefficients (see supplementary materials 6), estimation of this kinetic parameter for uptake of lasiocarpine and riddelliine into liver tissue based on partitioning between plasma and tissue is unlikely to affect the outcomes to a significant extent.

Given that the methods used in the present study and in the previous study (Chen et al., 2018) for predicting the in vivo liver toxicity of lasiocarpine and riddelliine in rat were similar, using species specific primary hepatocytes and species specific subcellular tissue fractions in similar in vitro incubations to define the species specific metabolic clearance, using a similar PBK model structure, the model predictions also provide insight in species differences in acute liver toxicity of lasiocarpine and riddelliine. This inter-species comparison, revealed that the predicted $\mathrm{BMDL}_{5}$ for liver toxicity of lasiocarpine and riddelliine in humans (average PoDs of Chinese and Caucasians) is up to 2.0-fold and8.2-fold lower, respectively, than that for rat, indicating that humans are somewhat more sensitive towards acute liver toxicity of lasiocarpine and riddelliine than rats. It is of interest to note that the in vitro cytotoxicity of these two compounds in human Caucasian hepatocytes was lower than in rat hepatocytes, and that the predicted higher toxicity in vivo thus results from inter-species differences in the kinetics. The kinetics showed a 1.9- and 5.0-fold slower clearance of lasiocarpine and riddelliine in humans (Caucasian) compared to rats. According to literature, the metabolism of PAs in rodents is mainly catalyzed by CYP 2B and CYP 3A subfamilies, while in humans, metabolism is mainly 
catalysed by CYP 3A4 (Li et al., 2011; Miranda et al., 1991). This observation is of interest when considering rat toxicity data for human risk assessment. Extrapolating rodent toxicity data to the human situation usually includes the use of a default uncertainty factor of 10 to account for species differences, consisting of a factor of 4.0 for kinetic and a factor of 2.5 for dynamic differences (WHO, 1999). In the present study, the inter-species differences in acute liver toxicity of lasiocarpine and riddelliine fall well within the default factor of 10 . Such a compound specific uncertainty factor that could be smaller than the default value of 10 was also reported for aristolochic acid I for which in vitro-PBK model facilitated reverse dosimetry-based predicted PoDs differed about 1.8-fold between rat and human and about 1.9-fold between mouse and human (Abdullah et al., 2016). Also for the bioactivation of estragole the compound specific uncertainty factor for differences in kinetics between humans and rats was predicted to be 2-fold and thus smaller than the default factor of 4 for kinetic differences (Punt et al., 2009). Altogether, these results reveal that the in vitro-PBK modelling-based reverse dosimetry may provide a way forward to define compound specific uncertainty factors, also called compound specific adjustment factors (CSAFs), in risk assessment.

Comparison of the predicted $\mathrm{BMDL}_{5}$ for lasiocarpine and riddelliine between Chinese and Caucasian subjects showed that for Chinese subjects, the predicted $\mathrm{BMDL}_{5}$ for lasiocarpine was 2.0-fold higher and for riddelliine 5.0-fold higher than for Caucasians, suggesting that Chinese subjects might be less sensitive towards acute hepatotoxicity of lasiocarpine and riddelliine. This is mainly due to the differences in bioactivation of the parent compound, resulting in less bioactivation for both compounds at similar dose levels in average Chinese compared to average Caucasian subjects, and occurs in spite of less efficient clearance in Chinese as compared to Caucasians. In the present study, human hepatocytes used to study the in vitro cytotoxicity of lasiocarpine and riddelliine were derived from Caucasian subjects. Since human hepatocytes derived from Chinese subjects are not available, in vitro cytotoxicity for Chinese hepatocytes was defined by read-across from the Caucasian liver hepatocytes taking into account the difference in bioactivation between Chinese and Caucasian liver microsomes, as reflected by 7-GS-DHP formation in in vitro incubations. In the present study, we used GSH as a trapping agent to estimate the amount of reactive metabolites formed in liver microsomal incubations which is in line with the method described previously (Tamta et al., 2012). It has been reported that dehydro-PAs react with GSH forming three metabolites, 7-GS-DHP, 9-GS-DHP and 7,9di-GSH-DHP, of which 7-GS-DHP is the major metabolite formed in vivo and in vitro (Chen et al., 2016; Lame et al., 1990; Lin et al., 1998; Reed et al., 1992; Tamta et al., 2012), whereas 7,9-di-GSDHP is only formed in vitro (Lin et al., 1998; Reed et al., 1992; Tamta et al., 2012). Other factors influencing the dynamics of liver toxicity caused by PAs, for example the relative potential for repair, cannot be taken into account in this way. Nevertheless the approach now taken provides an adequate first approach to study the potential ethnic differences in the toxicokinetic process of PA-induced liver 
liver toxicity caused by PAs, for example the relative potential for repair, cannot be taken into account in this way. Nevertheless the approach now taken provides an adequate first approach to study the potential ethnic differences in the toxicokinetic process of PA-induced liver toxicity.

The ethnic differences in metabolic clearance of lasiocarpine and riddelliine determined by substrate depletion using liver microsomess for both populations was 2- to 4-fold, with clearance by the average Caucasian being more efficient. The difference in efficiency for formation of bioactive metabolites for both compounds by the Chinese and Caucasian incubations amounted to 7- to 8-fold, the value for the average Caucasian being highest. It has been reported that besides CYP 450 enzymes, flavincontaining monooxygenases (FMO) and carboxylesterases are considered to be involved in detoxification pathways as shown in Figure 1 (Fu et al., 2004). FMO was found to be partly involved in the N-oxide formation and carboxylesterases are involved in hydrolysis of the ester groups of PAs leading to the formation of necine base and necic acid moieties, which is considered to be the major detoxification pathway ( $\mathrm{Fu}$ et al., 2004). Currently, two predominant carboxylesterases (CES) enzymes have been identified in human, i.e. CES 1 and CES 2 (Wang et al., 2018). A recent review reported that ethnic differences in carboxylesterases enzymes activity may be present due to single nucleotide polymorphisms (SNPs). For instance, CES 1 variants G1420E and D260fs, which are two important functional SNPs in Caucasian populations, showed reduced esterase activity in vitro, while these two variants were not found in the Asian populations (Cha et al., 2014; Wang et al., 2018). However, detailed studies of assessing ethnic differences in carboxylesterases activity are still lacking. It is also of interest to note that in toxicological risk assessment, a default uncertainty factor of 10 is used to account for inter-individual variation, which can be divided into a factor 3.16 for kinetics and 3.16 for dynamic differences (WHO, 1999). The ethnic human difference in predicted PoDs of lasiocarpine and riddelliine between Chinese and Caucasian subjects, were estimated to be 1.7 -and 5.0-fold respectively, and are thus in line with the default value of 3.16 for inter-individual kinetic differences. However, various studies report much higher than 2 to 4 -fold inter-individual variability in the expression of the major hepatic CYP enzyme (CYP 3A4) involved in PA metabolism, ranging up to 100-fold (Westlind-Johnsson et al., 2003), or even up to 400-fold when taking illness, inhibition, and induction-related interactions into account (Galetin et al., 2004; Wilkinson, 2005). Another study reported that inter-individual variation in the expression of CYP 3A4 varies 40- to 50-fold (IngelmanSundberg, 2004). Therefore, the human inter-individual variation in kinetics is likely to be larger than the default value of 3.16. In a next step, to actually quantify inter-individual human variation in sensitivity to acute liver toxicity of lasiocarpine and riddelliine, the PBK model of the present study should be developed for individual subjects and combined with Monte Carlo modelling to predict hepatotoxicity within the human population. This Monte Carlo modelling should especially consider the variability in the PBK model parameters shown in the sensitivity analysis to influence the model 
predictions to the largest extent including the $\mathrm{Ka}$ and the kinetic parameters for metabolic clearance in the liver.

Given that the ultimate critical effect of lasiocarpine and riddelliine is not only acute hepatotoxicity but also genotoxicity and subsequently, carcinogenic transformation, it may also be of interest to extent the current PBK model to a physiologically based dynamic (PBD) model able to predict in vivo DNA binding as previously done for estragole (Paini et al., 2010). This would require definition of an in vitro concentration response curve for DNA adduct formation in the primary hepatocytes, which can subsequently be translated to an in vivo dose-response curve for DNA adduct formation. According to the study of Xia et al. (2013), DNA adduct formation is a common biological biomarker of PAinduced tumorigenicity in rats. Previous studies reported that the metabolism of riddelliine by human liver microsomes resulted in a similar metabolic pattern and DNA adduct profile to those formed in the rat liver, suggesting that the mode of action of PAs studied in experimental rodents is highly relevant to humans (Xia et al., 2003; Zhao et al., 2012). The current PBK model could also translate in vitro concentration-response curves for DNA adduct formation in human liver cells to in vivo doseresponse curves for DNA adduct formation in the liver of human.

In conclusion, the present study shows that PBK modelling-based reverse dosimetry can identify the differences in inter-species and inter-ethnic human differences in liver toxicity of lasiocarpine and riddelliine. The species dependent variation in hepatotoxicity defined by the BMDL 5 (average PoDs of Caucasians and Chinese) is up to 2.0-fold for lasiocarpine and 8.2-fold for riddelliine, with humans being more susceptible to lasiocarpine and riddelliine-induced liver toxicity than rat. The inter-ethnic human difference between average Chinese and average Caucasian subjects was estimated to be 2.0fold for lasiocarpine and 5.0-fold for riddelliine, with the average Caucasian being more sensitive towards the acute liver toxicity of lasiocarpine and riddelliine, mainly due to more efficient reactive metabolite formation. Altogether, the present study shows proof-of-principle for a method to predict inter-species and inter-ethnic differences in in vivo liver toxicity for PAs by an alternative testing strategy integrating in vitro cytotoxicity assays with PBK modelling-based reverse dosimetry.

\section{Acknowledgements}

This work was funded by a grant from the China Scholarship Council (No. 201507720019 to NING JIA) 


\section{References}

Abdullah R, Alhusainy W, Woutersen J, Rietjens IMCM, Punt A., 2016. Predicting points of departure for risk assessment based on in vitro cytotoxicity data and physiologically based kinetic (PBK) modeling: The case of kidney toxicity induced by aristolochic acid I. Food Chem Toxicol 92:104-16

Adams IR, Hamlin KE, Jr., Jelinek CF, Phillips RF., 1942. Structure of riddelliine, the alkaloid in Senecio riddellii. J Am Chem Soc 64:2760-3

Bane A, Seboxa T, Mesfin G., 2012. An outbreak of veno-occlusive liver disease in northern Ethiopia, clinical findings. Ethiop Med J 50 Suppl 2:9-16

Barter ZE, Tucker GT, Rowland-Yeo K., 2013. Differences in Cytochrome P450-Mediated Pharmacokinetics Between Chinese and Caucasian Populations Predicted by Mechanistic Physiologically Based Pharmacokinetic Modelling. Clin Pharmacokinet 52(12):1085-1100

Bfr, 2013. Pyrrolizidine alkaloids in herbal teas and teas. BfR opinion

Brown RP, Delp MD, Lindstedt SL, Rhomberg LR, Beliles RP., 1997. Physiological parameter values for physiologically based pharmacokinetic models. Toxicol Ind Health 13(4):407-84

Cha Y-J, Jeong H-E, Shin J-G., 2014. Genetic Polymorphisms of the Carboxylesterase 1 ( CES1 ) Gene in a Korean Population. Transl Clin Pharmacol 22(2):30-34

Chan PC, Haseman JK, Prejean JD, Nyska A., 2003. Toxicity and carcinogenicity of riddelliine in rats and mice. Toxicol Lett 144(3):295-311

Chan SC, Liu CL, Lo CM., 2006. Estimating liver weight of adults by body weight and gender. World J Gastroenterol 12(14):2217-22

Chen L, Ning J, Louisse J, Wesseling S, Rietjens IMCM., 2018. Use of physiologically based kinetic modelling-facilitated reverse dosimetry to convert in vitro cytotoxicity data to predicted in vivo liver toxicity of lasiocarpine and riddelliine in rat. Food Chem Toxicol 116(Pt B):216226

Chen M, Li L, Zhong D, Shen S, Zheng J, Chen X., 2016. 9-Glutathionyl-6,7-dihydro-1hydroxymethyl-5H-pyrrolizine Is the Major Pyrrolic Glutathione Conjugate of RetronecineType Pyrrolizidine Alkaloids in Liver Microsomes and in Rats. Chem Res Toxicol 29(2):1809

Cubitt HE, Houston JB, Galetin A., 2009. Relative importance of intestinal and hepatic glucuronidation-impact on the prediction of drug clearance. Pharm Res 26(5):1073-83

Culvenor CC., 1983. Estimated intakes of pyrrolizidine alkaloids by humans. A comparison with dose rates causing tumors in rats. J Toxicol Environ Health 11(4-6):625-35

DeJongh J, Verhaar HJM, Hermens JLM., 1997. A quantitative property-property relationship (QPPR) approach to estimate in vitro tissue-blood partition coefficients of organic chemicals in rats and humans. Arch Toxicol 72(1):17-25

EFSA, 2011. Scientific opinion on pyrrolizidine alkaloids in food and feed. EFSA Journal 9(11)

EFSA, 2017. Risks for human health related to the presence of pyrrolizidine alkaloids in honey, tea, herbal infusions and food supplements. EFSA Journal 15(7)

EPA, 2017. U.S. EPA Benchmark dose modeling guidance.

Evans MV, Andersen ME., 2000. Sensitivity analysis of a physiological model for 2,3,7,8tetrachlorodibenzo-p-dioxin (TCDD): assessing the impact of specific model parameters on sequestration in liver and fat in the rat. Toxicol Sci 54(1):71-80

Fashe MM, Juvonen RO, Petsalo A, Rasanen J, Pasanen M., 2015. Species-Specific Differences in the in Vitro Metabolism of Lasiocarpine. Chem Res Toxicol 28(10):2034-44

Field RA, Stegelmeier BL, Colegate SM, Brown AW, Green BT., 2015. An in vitro comparison of the cytotoxic potential of selected dehydropyrrolizidine alkaloids and some N-oxides. Toxicon 97:36-45

Fu PP, Xia Q, Lin G, Chou MW., 2004. Pyrrolizidine Alkaloids - Genotoxicity, Metabolism Enzymes, Metabolic Activation, and Mechanisms. Drug Metab Rev 36(1):1-55 
Galetin A, Brown C, Hallifax D, Ito K, Houston JB., 2004. Utility of recombinant enzyme kinetics in prediction of human clearance: Impact of variability, CYP3A5, and CYP2C19 on CYP3A4 probe substrates. Drug Metab Dispos 32(12):1411-1420

Gerets HHJ, Tilmant K, Gerin B., 2012. Characterization of primary human hepatocytes, HepG2 cells, and HepaRG cells at the mRNA level and CYP activity in response to inducers and their predictivity for the detection of human hepatotoxins. Cell Biology and Toxicology 28(2):6987

Hou TJ, Zhang W, Xia K, Qiao XB, Xu XJ., 2004. ADME evaluation in drug discovery. 5. Correlation of Caco-2 permeation with simple molecular properties. J Chem Inf Comput Sci 44(5):1585600

IARC, 2017. Agents Classified by the IARC Monographs. vol 1-118. International Agency for Research on Cancer, Lyon, France

Ingelman-Sundberg M., 2004. Human drug metabolising cytochrome P450 enzymes: properties and polymorphisms. Naunyn Schmiedebergs Arch Pharmacol 369(1):89-104

Kanebratt KP, Andersson TB., 2008. Evaluation of HepaRG cells as an in vitro model for human drug metabolism studies. Drug Metab Dispos 36(7):1444-1452

Kvist AJ, Kanebratt KP, Walentinsson A., 2018. Critical differences in drug metabolic properties of human hepatic cellular models, including primary human hepatocytes, stem cell derived hepatocytes, and hepatoma cell lines. Biochem Pharmacol 155:124-140

Lambert CB, Spire C, Claude N, Guillouzo A., 2009. Dose- and time-dependent effects of phenobarbital on gene expression profiling in human hepatoma HepaRG cells. Toxicol Appl Pharmacol 234(3):345-60

Lame MW, Morin D, Jones AD, Segall HJ, Wilson DW., 1990. Isolation and identification of a pyrrolic glutathione conjugate metabolite of the pyrrolizidine alkaloid monocrotaline. Toxicol Lett 51(3):321-9

Li N, Xia QS, Ruan JQ, Fu PP, Lin G., 2011. Hepatotoxicity and Tumorigenicity Induced by Metabolic Activation of Pyrrolizidine Alkaloids in Herbs. Curr Drug Metab 12(9):823-834

Lin G, Cui YY, Hawes EM., 1998. Microsomal formation of a pyrrolic alcohol glutathione conjugate of clivorine. Firm evidence for the formation of a pyrrolic metabolite of an otonecine-type pyrrolizidine alkaloid. Drug Metab Dispos 26(2):181-4

Lin KM, Lau JK, Smith R, Phillips P, Antal E, Poland RE., 1988. Comparison of alprazolam plasma levels in normal Asian and Caucasian male volunteers. Psychopharmacology 96(3):365-9

Lubberstedt M, Muller-Vieira U, Mayer M., 2011. HepaRG human hepatic cell line utility as a surrogate for primary human hepatocytes in drug metabolism assessment in vitro. J Pharmacol Toxicol 63(1):59-68

Medinsky MA, Leavens TL, Csanady GA, Gargas ML, Bond JA., 1994. In vivo metabolism of butadiene by mice and rats: a comparison of physiological model predictions and experimental data. Carcinogenesis 15(7):1329-40

Miranda CL, Reed RL, Guengerich FP, Buhler DR., 1991. Role of cytochrome P450IIIA4 in the metabolism of the pyrrolizidine alkaloid senecionine in human liver. Carcinogenesis 12(3):515-9

Mohabbat O, Younos MS, Merzad AA, Srivastava RN, Sediq GG, Aram GN., 1976. An outbreak of hepatic veno-occlusive disease in north-western Afghanistan. Lancet 2(7980):269-71

NHFPC, 2007. Reference individuals for use in radiation protection-Part2: Masses of main organs and tissues. National Health and Family Planning Commission of the People's Republic of China

NHFPC, 2014. Reference individiuals for use in radiation protection-Part3: main physiological parameters. vol GBZ/T 200.3. National Health and Family Planning Commission of the People's Republic of China

Ning J, Louisse J, Spenkelink B, Wesseling S, Rietjens IMCM., 2017. Study on inter-ethnic human differences in bioactivation and detoxification of estragole using physiologically based kinetic modeling. Arch Toxicol 91(9):3093-3108

NTP , 1978. Bioassay of lasiocarpine for possible carcinogenicity. Natl Cancer Inst Carcinog Tech Rep Ser 39:1-66

NTP, 2003. Toxicology and carcinogenesis studies of riddelliine (CAS No. 23246-96-0) in F344/N rats and B6C3F1 mice (gavage studies). Natl Toxicol Program Tech Rep Ser(508) 
Paini A, Punt A, Viton F., 2010. A physiologically based biodynamic (PBBD) model for estragole DNA binding in rat liver based on in vitro kinetic data and estragole DNA adduct formation in primary hepatocytes. Toxicol Appl Pharmacol 245(1):57-66

Punt A, Paini A, Boersma MG., 2009. Use of physiologically based biokinetic (PBBK) modeling to study estragole bioactivation and detoxification in humans as compared with male rats. Toxicol Sci 110(2):255-69.

Reed RL, Miranda CL, Kedzierski B, Henderson MC, Buhler DR., 1992. Microsomal formation of a pyrrolic alcohol glutathione conjugate of the pyrrolizidine alkaloid senecionine. Xenobiotica 22(11):1321-7

Shi ZR, Yan LN, Li B, Wen TF., 2009. Evaluation of standard liver volume formulae for Chinese adults. World J Gastroenterol 15(32):4062-6

Slob W., 2002. PROAST: Software for dose-response modeling and benchmark dose analysis. RIVM.

Smith LW, Culvenor CCJ., 1981. Plant sources of hepatotoxic pyrrolizidine alkaloids. J Nat Prod 44(2):129-52.

Stegelmeier BL, Edgar JA, Colegate SM., 1999. Pyrrolizidine alkaloid plants, metabolism and toxicity. J Nat Toxins 8(1):95-116

Tamta H, Pawar RS, Wamer WG, Grundel E, Krynitsky AJ, Rader JI., 2012. Comparison of metabolism-mediated effects of pyrrolizidine alkaloids in a HepG2/C3A cell-S9 co-incubation system and quantification of their glutathione conjugates. Xenobiotica 42(10):1038-48

Tandon HD, Tandon BN, Mattocks AR., 1978. An epidemic of veno-occlusive disease of the liver in Afghanistan. Pathologic features. Am J Gastroenterol 70(6):607-13

Tu M, Sun S, Wang K., 2013. Organic cation transporter 1 mediates the uptake of monocrotaline and plays an important role in its hepatotoxicity. Toxicology 311(3):225-30

van Liempd S, Morrison D, Sysmans L, Nelis P, Mortishire-Smith R., 2011. Development and validation of a higher-throughput equilibrium dialysis assay for plasma protein binding. J Lab Autom 16(1):56-67

Wang D, Zou L, Jin Q, Hou J, Ge G, Yang L., 2018. Human carboxylesterases: a comprehensive review. Acta Pharmaceutica Sinica B (In press)

Waters NJ, Jones R, Williams G, Sohal B., 2008. Validation of a rapid equilibrium dialysis approach for the measurement of plasma protein binding. J Pharm Sci 97(10):4586-95

Westlind-Johnsson A, Malmebo S, Johansson A., 2003. Comparative analysis of CYP3A expression in human liver suggests only a minor role for CYP3A5 in drug metabolism. Drug Metab Dispos 31(6):755-761

WHO, 1999. International Programme on Chemical Safety (IPCS). Principles for the assessment of risks to human health from exposure to chemicals. Geneva : World Health Organization.

Wiedenfeld H, Edgar J., 2011. Toxicity of pyrrolizidine alkaloids to humans and ruminants. Phytochem Rev 10(1):137-151

Wilkinson GR., 2005. Drug therapy - Drug metabolism and variability among patients in drug response. New Engl J Med 352(21):2211-2221

Xia Q, Chou MW, Kadlubar FF, Chan PC, Fu PP., 2003. Human liver microsomal metabolism and DNA adduct formation of the tumorigenic pyrrolizidine alkaloid, riddelliine. Chem Res Toxicol 16(1):66-73

Xia Q, Zhao Y, Von Tungeln LS., 2013. Pyrrolizidine alkaloid-derived DNA adducts as a common biological biomarker of pyrrolizidine alkaloid-induced tumorigenicity. Chem Res Toxicol 26(9):1384-96

Yang JL, He MM, Niu W., 2012. Metabolic capabilities of cytochrome P450 enzymes in Chinese liver microsomes compared with those in Caucasian liver microsomes. Brit $\mathrm{J}$ Clin Pharmaco 73(2):268-284

Yuan D, Lu T, Wei YG., 2008. Estimation of standard liver volume for liver transplantation in the Chinese population. Transplant Proc 40(10):3536-40

Zhao Y, Xia Q, Gamboa da Costa G, Yu H, Cai L, Fu PP., 2012. Full structure assignments of pyrrolizidine alkaloid DNA adducts and mechanism of tumor initiation. Chem Res Toxicol 25(9):1985-96 


\section{Supplementary materials 1}

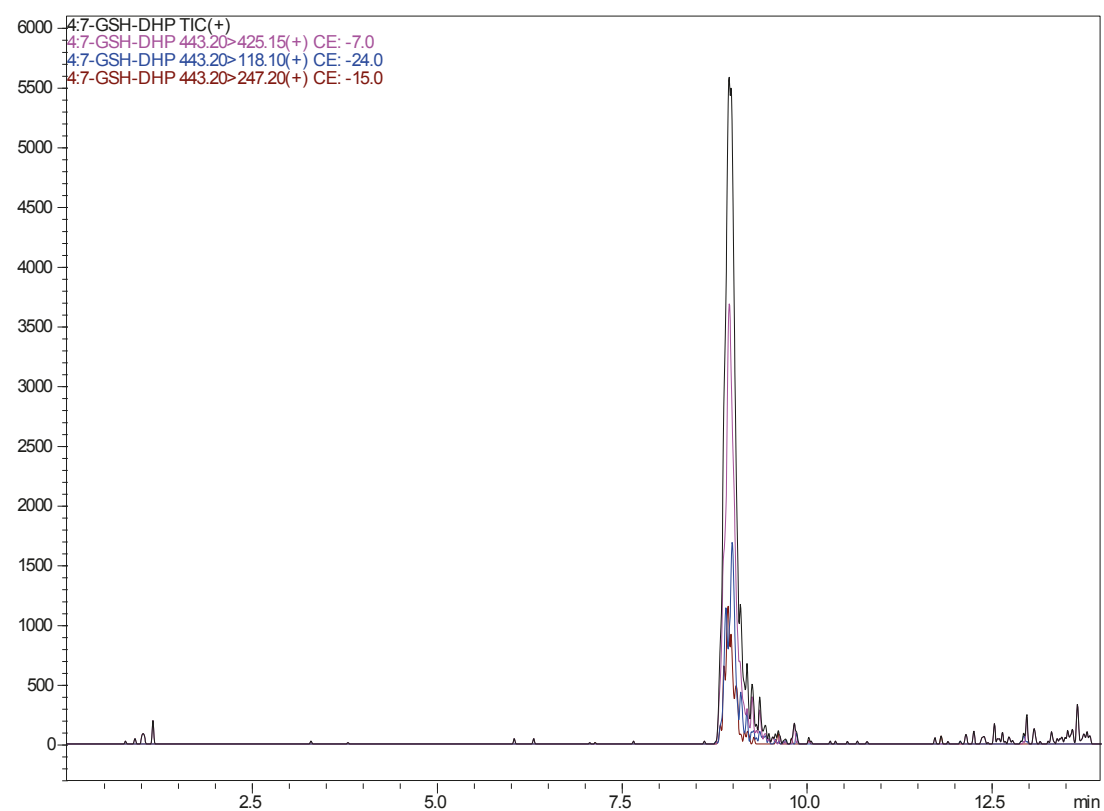

Fig. S1 LC-MS chromatogram of 7-GSH-DHP formed in the incubation of $200 \mu \mathrm{M}$ lasiocarpine with Caucasian liver microsomes in the presence of $2 \mathrm{mM}$ NADPH and $20 \mathrm{mM}$ GSH for $30 \mathrm{~min}$ 


\section{Supplementary materials 2}

Table S2 Parameters used in the PBK model for lasiocarpine and riddelliine in Chinese and Caucasian subjects as obtained from literature.

\begin{tabular}{|c|c|c|}
\hline Model parameters & Chinese $^{\mathrm{a}, \mathrm{c}}$ & Caucasian $^{\mathrm{b}, \mathrm{c}}$ \\
\hline \multicolumn{3}{|l|}{ Physiological parameters } \\
\hline Bodyweight (kg) & 60 & 70 \\
\hline \multicolumn{3}{|l|}{ Tissue volumes ( $\%$ of body weight) } \\
\hline Fat & 18.7 & 21.4 \\
\hline Liver & 2.3 & 2.6 \\
\hline Small intestine & 0.5 & 0.9 \\
\hline Richly perfused & 4.8 & 4.1 \\
\hline Slowly perfused & 62.3 & 59.6 \\
\hline Arterial blood & 2.0 & 2.0 \\
\hline Venous blood & 5.9 & 5.9 \\
\hline Cardiac output (L/hr/kg bw $\left.{ }^{0.74}\right)$ & 15 & 15 \\
\hline \multicolumn{3}{|l|}{ Percentage of cardiac output } \\
\hline Fat & 6.75 & 5.2 \\
\hline Liver & 26.3 & 22.7 \\
\hline Small intestine & 10.5 & 18.1 \\
\hline Richly perfused & 33.2 & 43.7 \\
\hline Slowly perfused & 23.3 & 23.3 \\
\hline \multicolumn{3}{|l|}{ Tissue: blood partition coefficients } \\
\hline \multicolumn{3}{|l|}{ Lasiocarpine } \\
\hline Fat/blood & 3.46 & 3.46 \\
\hline Liver/blood & 0.78 & 0.78 \\
\hline Small intestine/blood & 0.78 & 0.78 \\
\hline Richly perfused/blood & 0.78 & 0.78 \\
\hline Slowly perfused/blood & 0.86 & 0.86 \\
\hline
\end{tabular}




\begin{tabular}{lll}
\hline Riddelliine & \\
Fat/blood & 0.25 & 0.25 \\
Liver/blood & 0.63 & 0.63 \\
Small intestine/blood & 0.63 & 0.63 \\
Richly perfused/blood & 0.63 & 0.63 \\
Slowly perfused/blood & 0.77 & 0.77
\end{tabular}

${ }^{a}$ NHFPC (2007); NHFPC (2014)

${ }^{b}$ Brown et al. (1997)

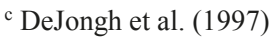




\section{Supplementary materials 3}

;Physiological parameters

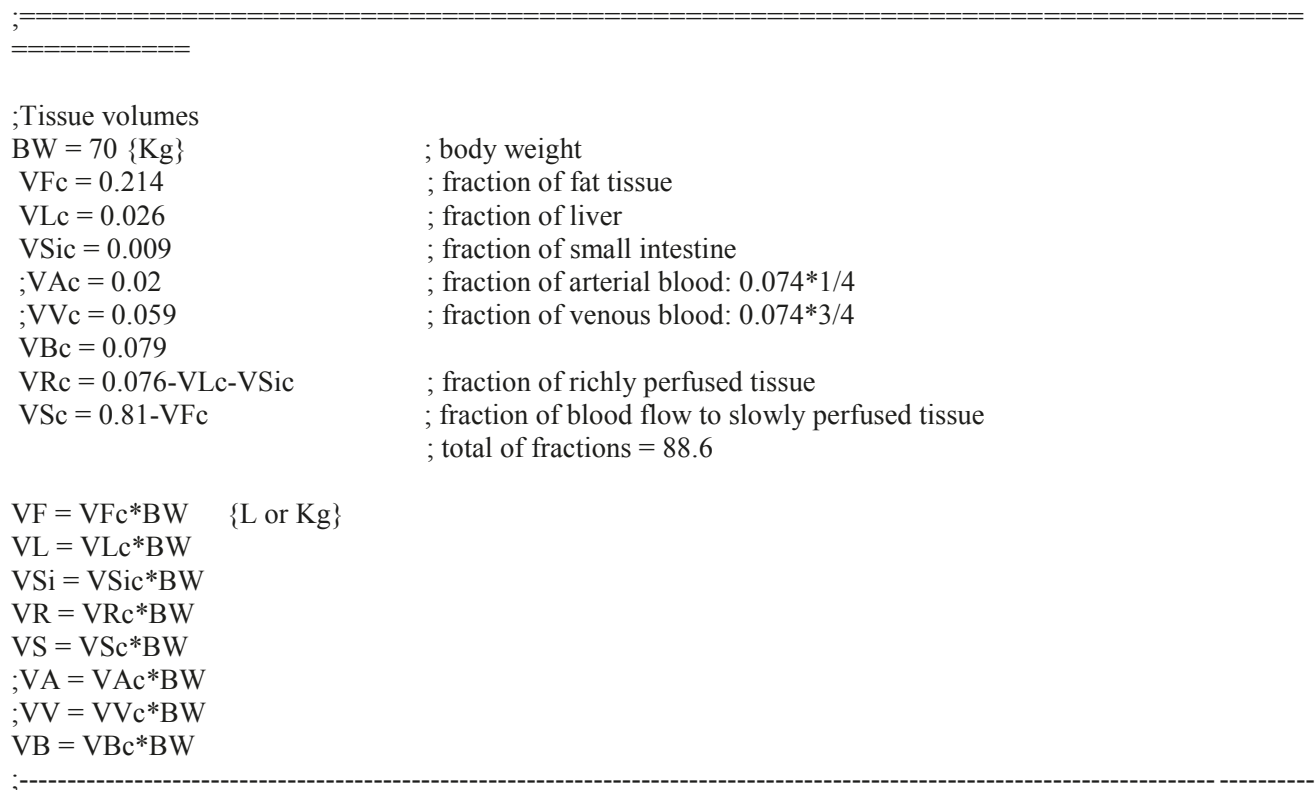

;Blood flow rates

$\mathrm{QC}=15^{*} \mathrm{BW}^{\wedge} 0.74\{\mathrm{~L} / \mathrm{hr}\}$; Info: $\mathrm{QC}=15^{*} \mathrm{BW}^{\wedge} 0.74$

$\mathrm{QFc}=0.052$

$\mathrm{QLc}=0.227-\mathrm{QSic}$

$\mathrm{QSic}=0.181$

; Fraction of blood flow to fat

; Fraction of blood flow to liver

$\mathrm{QRc}=0.7-\mathrm{QLc}-\mathrm{QSic}$

; Fraction of blood flow to small intestine

$\mathrm{QSc}=0.3-\mathrm{QFc}$

; Fraction of blood flow to richly perfused tissue

; Fraction of blood flow to slowly perfused tissue ; total of fractions $=1$

$\mathrm{QF}=\mathrm{QFc} * \mathrm{QC}\{\mathrm{L} / \mathrm{hr}\}$

$\mathrm{QL}=\mathrm{QLc} * \mathrm{QC}$

$\mathrm{QSi}=\mathrm{QSic} * \mathrm{QC}$

$\mathrm{QR}=\mathrm{QRc} * \mathrm{QC}$

$\mathrm{QS}=\mathrm{QSc} * \mathrm{QC}$

;Partition Coefficients

;lasiocarpine

PLL $=0.78$

$\mathrm{PFL}=3.46$

$\mathrm{PRL}=0.78$

$\mathrm{PSL}=0.86$

$\mathrm{PIL}=0.78$

; liver/blood partition coefficient

; fat/blood partition coefficient

; richly perfused tissues/blood partition coefficient

; slowly perfused tissues/blood partition coefficient

; intestine/blood partition coefficient 
;Biochemical parameters

;Linear uptake rate $(\mathrm{hr}-1)$

$\mathrm{Ka}=1.55$

; The averaged Ka values reported by Chen et al., 2018. The Ka value was based on 1. Papp predicted by Schrodinger and 2. Papp derived from equation log Papp $=-5.469+0.236 \log \mathrm{P}$ (Hou et al., 2004)

;Metabolism liver

;Scaling factors

MPL $=35$

;Liver microsomal protein yield (mg/gram liver)

$\mathrm{L}=\mathrm{VLC} * 1000 \quad ; \mathrm{Liver}=34(\mathrm{gram} / \mathrm{kg} \mathrm{BW})$

;metabolites of lasiocarpine, unscaled maximum rate of metabolism (nmol min-1 (mg protein)-1)

VmaxLM1c $=5.107$ ; based on substrate depletion in Caucasian liver microsome

;metabolites of lasiocarpine, scaled maximum rate of metabolism (umol hr-1)

VMaxLM1 $=$ VMaxLM1c/1000*60*MPL*L*BW

;metabolites of lasiocarpine, affinity constants (umol/L)

$\mathrm{KmLM1}=25.81 \quad$; based on substrate depletion in Caucasian liver microsome

;Metabolism small intestine

;Scaling factor

$\mathrm{MPSi}=20.6 \quad$;Small intestine microsome fraction yield (mg/g small intestine)

$\mathrm{Si}=\mathrm{VSiC} * 1000$

;metabolites of lasiocarpine, unscaled maximum rate of metabolism (nmol min-1 (mg)-1)

VmaxSiM2c $=0.8840 \quad$; based on the substrate depletion in Caucasian intestine microsome

;metabolites of lasiocarpine, scaled maximum rate of metabolism (umol hr-1)

VMaxSiM2 $=$ VMaxSiM2c/1000*60*MPSi*SI*BW

;metabolites of lasiocarpine, affinity constants (umol/L)

$\mathrm{KmSiM}=72.12$; based on the substrate depletion in Caucasian intestine microsome

;Run settings

;Molecular weight 
$\mathrm{MWL}=411.2$

;Given dose (mg/ kg bw) and oral dose umol/ kg bw
GDOSE $=10\{\mathrm{mg} / \mathrm{kg} \mathrm{bw}\}$
; GDOSE $=$ given dose

bw

ODOSE $=$ GDOSE $* 1 \mathrm{E}-3 / \mathrm{MWL} * 1 \mathrm{E} 6\{\mathrm{umol} / \mathrm{kg}$ bw $\} ;$ ODOSE $=$ given dose recalculated to umol $/ \mathrm{kg}$
DOSE $=$ ODOSE $*$ BW;
; DOSE $=$ umol

;Time
Starttime $=0$;
Stoptime $=24$;
in hrs
in hrs

\section{;Dynamics}

;slowly perfused tissue compartment
;AS = Amount lasiocarpine in slowly perfused tissue, umol
$\mathrm{AS}^{\prime}=\mathrm{QS}^{*}(\mathrm{CB}-\mathrm{CVS})$
Init $\mathrm{AS}=0$
$\mathrm{CS}=\mathrm{AS} / \mathrm{VS}$
$\mathrm{CVS}=\mathrm{CS} / \mathrm{PSL}$

;richly perfused tissue compartment
$; \mathrm{AR}=$ Amount lasiocarpine in richly perfused tissue, umol
$\mathrm{AR}^{\prime}=\mathrm{QR}^{*}(\mathrm{CB}-\mathrm{CVR})$
Init $\mathrm{AR}=0$
$\mathrm{CR}=\mathrm{AR} / \mathrm{VR}$
$\mathrm{CVR}=\mathrm{CR} / \mathrm{PRL}$

;fat compartment

;AF $=$ Amount lasiocarpine in fat tissue, umol

$\mathrm{AF}^{\prime}=\mathrm{QF}^{*}(\mathrm{CB}-\mathrm{CVF})$

Init $\mathrm{AF}=0$

$\mathrm{CF}=\mathrm{AF} / \mathrm{VF}$

$\mathrm{CVF}=\mathrm{CF} / \mathrm{PFL}$

;uptake lasiocarpine from GI tract

;AGI $=$ Amount lasiocarpine remaining in GI tract (umol)

AGI' =-ka*AGI

Init $\mathrm{AGI}=$ dose

;liver compartment

$; \mathrm{AL}=$ Amount lasiocarpine in liver tissue, umol

$\mathrm{AL}^{\prime}=\mathrm{QL} * \mathrm{CB}+\mathrm{QSi}{ }^{*} \mathrm{CVSi}-(\mathrm{QL}+\mathrm{QSi}){ }^{*} \mathrm{CVL}-\mathrm{AMLM} 1{ }^{\prime}$

Init $\mathrm{AL}=0$

$\mathrm{CL}=\mathrm{AL} / \mathrm{VL}$

$\mathrm{CVL}=\mathrm{CL} / \mathrm{PLL}$ 
;AMLM1=Amount lasiocarpine metabolized in liver to metabolite 1, umol

AMLM1' $=$ VmaxLM1*CVL/ KmLM1 + CVL $)$

Init AMLM1 $=0$

;small intestine compartment

;ASi $=$ Amount lasiocarpine in small intestine tissue, umol

$\mathrm{ASi}^{\prime}=\mathrm{QSi}{ }^{*}(\mathrm{CB}-\mathrm{CVSi})+\mathrm{ka} * \mathrm{AGI}-\mathrm{AMSiM}{ }^{\prime}$

Init $\mathrm{ASi}=0$

$\mathrm{CSi}=\mathrm{ASi} / \mathrm{VSi}$

$\mathrm{CVSi}=\mathrm{CSi} / \mathrm{PIL}$

;AMSiM2=Amount lasiocarpine metabolized in small intestine to metabolites2, umol

AMSiM2'= VmaxSiM2*CVSi $/(\mathrm{KmSiM} 2+\mathrm{CVSi})$

Init AMSiM2 $=0$

; arterial blood compartment

$; \mathrm{CA}=$ Concentration arterial blood lasiocarpine

$; \mathrm{CA}=\mathrm{CV}$

; venous blood compartment

; $\mathrm{CB}=$ Concentration venous blood lasiocarpine (umol/L)

$\mathrm{AB}^{\prime}=(\mathrm{QF} * \mathrm{CVF}+(\mathrm{QL}+\mathrm{QSi}) * \mathrm{CVL}+\mathrm{QR} * \mathrm{CVR}+\mathrm{QS} * \mathrm{CVS}-\mathrm{QC} * \mathrm{CB})$

Init $\mathrm{AB}=0$

$\mathrm{CB}=\mathrm{AB} / \mathrm{VB}$

$\mathrm{AUC}^{\prime}=\mathrm{CB}$

init $\mathrm{AUC}=0$

;Mass balance calculations

\{Mass Balance\}

Total $=$ DOSE

Calculated $=\mathrm{AF}+\mathrm{AS}+\mathrm{AR}+\mathrm{AL}+\mathrm{AB}+\mathrm{AGI}+\mathrm{ASI}+\mathrm{AMLM} 1+\mathrm{AMSiM} 2$

$\mathrm{ERROR}=(($ Total-Calculated $) /$ Total $+1 \mathrm{E}-30) * 100$

MASSBBAL $=$ Total-Calculated +1

PercAMLM1 $=($ AMLM1 $) * 100 /$ DOSE

PercAMSiM2 $=($ AMSiM2 $) * 100 /$ DOSE

PercAL $=\mathrm{AL} * 100 / \mathrm{DOSE}$

; blood concentration in $\mathrm{ng} / \mathrm{ml}$

$\mathrm{CBngmL}=\mathrm{CB} * \mathrm{MWL}$ 


\section{Supplementary materials 4}

Table S4 In vitro cytotoxicity data of lasiocarpine and riddelliine based on the experiments of the present study

\begin{tabular}{|c|c|c|c|c|c|}
\hline Compound & Cell model & $\begin{array}{l}\text { Compound } \\
\text { concentration }(\mu \mathrm{M})\end{array}$ & $\begin{array}{l}\text { Mean of cell } \\
\text { viability } \%\end{array}$ & $\begin{array}{l}\text { The effect } \\
\text { concentration in } \\
\text { human liver blood } \\
(\mu \mathrm{M})\end{array}$ & $\begin{array}{l}\text { Dose reconstructed } \\
\text { using PBK model } \\
(\mathrm{mg} / \mathrm{kg} \mathrm{bw})\end{array}$ \\
\hline \multirow{3}{*}{ Lasiocarpine } & HepaRG & $\begin{array}{l}0,4.7,9.4,18.8 \\
37.5,75,150,300\end{array}$ & $\begin{array}{l}100,108, \\
108,89,93 \\
75,52,40\end{array}$ & $\begin{array}{l}0,10.9,21.8,43.6, \\
87.2,174.4,348.8, \\
697.7\end{array}$ & $\begin{array}{l}0,27.7,43.5,63.2, \\
86.4,116.8,164.3, \\
248.4\end{array}$ \\
\hline & $\begin{array}{l}\text { Human } \\
\text { (Caucasian) } \\
\text { hepatocyte }\end{array}$ & $\begin{array}{l}0,4.7,9.4,18.8 \\
37.5,75,150,300\end{array}$ & $\begin{array}{l}100,82,62 \\
52,33,26 \\
20,17\end{array}$ & $\begin{array}{l}0,10.9,21.8,43.6, \\
87.2,174.4,348.8, \\
697.7\end{array}$ & $\begin{array}{l}0,27.7,43.5,63.2 \\
86.4,116.8,164.3 \\
248.4\end{array}$ \\
\hline & $\begin{array}{l}\text { Corrected } \\
\text { human } \\
\text { (Chinese) } \\
\text { hepatocyte }\end{array}$ & $\begin{array}{l}0,34,70,104,230 \\
351,707,1414\end{array}$ & $\begin{array}{l}100,82,62 \\
52,33,26 \\
20,17\end{array}$ & $\begin{array}{l}0,79.3,160.8, \\
242.5,535.9, \\
817.6,1645.2, \\
3290.5\end{array}$ & $\begin{array}{l}0,62.0,93.7,120.4, \\
202.2,276.5,489.6, \\
904.1\end{array}$ \\
\hline \multirow{3}{*}{ Riddelliine } & HepaRG & $\begin{array}{l}0,4.7,9.4,18.8 \\
37.5,75,150,300\end{array}$ & $\begin{array}{l}100,90,81 \\
80,70,67 \\
49,37\end{array}$ & $\begin{array}{l}0,10.9,21.8,43.6, \\
87.2,174.4,348.8, \\
697.7\end{array}$ & $\begin{array}{l}0,6.0,11.5,22.9, \\
43.9,80.8,144.6, \\
253.7\end{array}$ \\
\hline & $\begin{array}{l}\text { Human } \\
\text { (Caucasian) } \\
\text { hepatocyte }\end{array}$ & $\begin{array}{l}0,4.7,9.4,18.8 \\
37.5,75,150,300\end{array}$ & $\begin{array}{l}100,80,71, \\
63,54,40 \\
32,29\end{array}$ & $\begin{array}{l}0,10.9,21.8,43.6, \\
87.2,174.4,348.8, \\
697.7\end{array}$ & $\begin{array}{l}0,6.0,11.5,22.9, \\
43.9,80.8,144.6 \\
253.7\end{array}$ \\
\hline & $\begin{array}{l}\text { Corrected } \\
\text { Human } \\
\text { (Chinese) } \\
\text { hepatocyte }\end{array}$ & $\begin{array}{l}0,39.3,85.5,192.8, \\
307.4,618.3, \\
1236.7,2236.1\end{array}$ & $\begin{array}{l}100,80,71, \\
63,54,40 \\
32,29\end{array}$ & $\begin{array}{l}0,91.4,198.7, \\
448.4,714.9, \\
1438.0,2875.9, \\
5200.2\end{array}$ & $\begin{array}{l}0,28.3,58.0,122.4, \\
187.3,359.8,694.1, \\
1230.1\end{array}$ \\
\hline
\end{tabular}




\section{Supplementary materials 5}

\section{Lasiocarpine}

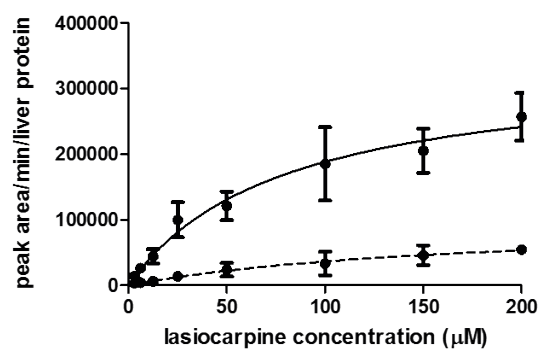

Riddelliine

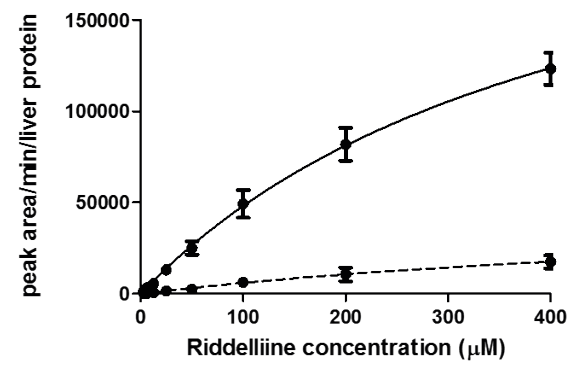

Fig. S5 lasiocarpine and riddelliine concentration-dependent rate of formation of 7-GSH-DHP by liver in incubations with Chinese (dotted line) and Caucasian (solid line) liver microsomes in the presence of the cofactor NADPH. Data points represent mean values \pm SD of three individual experiments

Table S5 Fold-differences in 7-GSH-DHP formation in incubation with Chinese and Caucasian liver microsomes at each substrate concentration.

\begin{tabular}{|c|c|c|c|}
\hline & \multirow{2}{*}{$\begin{array}{l}\text { Substrate concentration } \\
\qquad(\mu \mathrm{M})\end{array}$} & \multicolumn{2}{|c|}{ Fold-difference $\pm \mathrm{SD}^{3}$} \\
\hline & & Lasiocarpine & Riddelliine \\
\hline & 3.1 & $4.5 \pm 0.4$ & $5.1 \pm 0.6$ \\
\hline & 6.2 & $6.4 \pm 0.6$ & $10.2 \pm 2.9$ \\
\hline & 12.5 & $7.2 \pm 1.0$ & $8.4 \pm 0.9$ \\
\hline & 25 & $7.3 \pm 1.5$ & $9.1 \pm 2.6$ \\
\hline & 50 & $5.5 \pm 1.7$ & $10.3 \pm 0.8$ \\
\hline & 100 & $6.2 \pm 1.8$ & $8.2 \pm 0.8$ \\
\hline & $150^{a} / 200^{b}$ & $4.7 \pm 1.1$ & $8.2 \pm 1.1$ \\
\hline & $200^{\mathrm{a}} / 400^{\mathrm{b}}$ & $4.7 \pm 0.2$ & $7.5 \pm 0.6$ \\
\hline asiocarpine concen & tion & & \\
\hline Riddelliine concentr & & & \\
\hline
\end{tabular}




\section{Supplementary materials 6}

\section{Lasiocarpine}

a

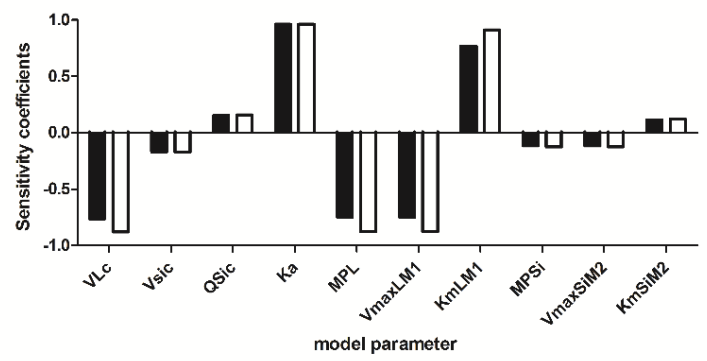

b

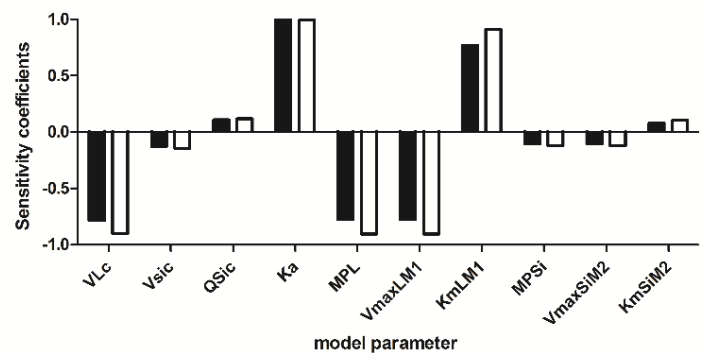

Riddelliine
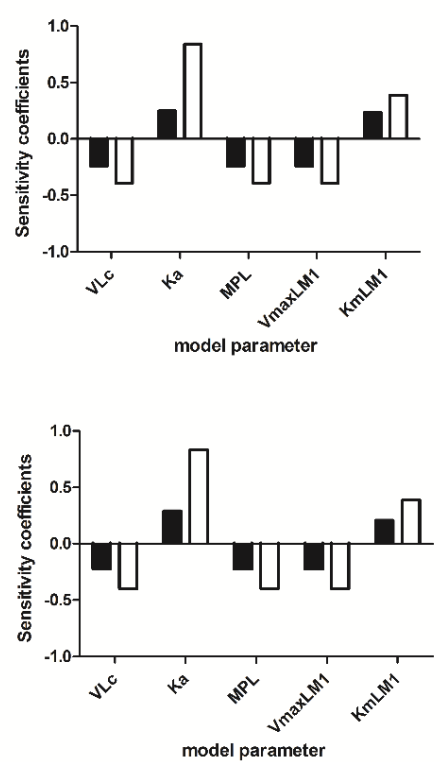

Fig. S6 Normalized sensitivity coefficients of model parameters for the prediction of $\mathrm{C}_{\max }$ of lasiocarpine and riddelliine in Chinese (black bar) and Caucasian (white bar) liver after oral administration to $8 \mathrm{ng} / \mathrm{kg}$ bw (a) and $3 \mathrm{mg} / \mathrm{kg}$ bw (b) of both compounds. VLc=fraction of liver tissue, VSic=fraction of small intestine tissue, $\mathrm{QSic}=$ fraction of blood flow to small intestine, $\mathrm{Ka}=$ absorption rate constant, $\mathrm{MPL}=$ microsomal protein yield in liver, MPSi=microsomal protein yield in small intestine. The $\mathrm{V}_{\max } \mathrm{LM} 1 / \mathrm{SiM} 2$ and $\mathrm{K}_{\mathrm{m}} \mathrm{LM} 1 / \mathrm{SiM} 2$ represent the maximum rate of formation and Michaelis-Menten constant for the substrate depletion in the liver/small intestine 


\section{Supplementary materials 7}

PROAST analysis

BMD analysis was performed using the PROAST software from The National Institute for Public Health and the Environment of the Netherlands (RIVM) version 38.9 (Slob, 2002) using Exponential and Hill models for continuous data. The model was selected if the fitted model passes the goodness-of-fit test at $\mathrm{P}=0.05$ and the ratio of BMD and BMDL should not be more than 3-fold (EPA, 2017).

We performed BMD modelling for both ethnic populations on the predicted dose-response data for lasiocarpine and riddelliine based on PBK modelling-based reverse dosimetry of cytotoxicity data using human hepatocytes.

Table S7.1 Results from the BMD analysis using PROAST software of the predicted dose-response data of lasiocarpine for the average Chinese using PBK modelling-based reverse dosimetry of cytotoxicity data derived from human hepatocytes. The table presents the $\mathrm{BMD}_{5}, \mathrm{BMDL}_{5}$ and $\mathrm{BMDU}_{5}$ for a $\mathrm{BMR}$ (Benchmark response) of $5 \%$ compared to the control group, with characteristics of the model fit for Hill 5.

\begin{tabular}{|l|l|l|l|l|l|}
\hline Model & BMR & Log-likelihood & $\begin{array}{l}\text { BMD } \\
(\mathrm{mg} / \mathrm{kg} \text { bw })\end{array}$ & $\begin{array}{l}\text { BMDL5 } \\
(\mathrm{mg} / \mathrm{kg} \text { bw })\end{array}$ & $\begin{array}{l}\mathrm{BMDU}_{5} \\
(\mathrm{mg} / \mathrm{kg} \text { bw })\end{array}$ \\
\hline Full & $5 \%$ & -5.7 & NA & NA & NA \\
\hline $\begin{array}{l}\text { Hill 5-CED } \\
\mathrm{a}^{*}\left(1+(\mathrm{c}-1) \mathrm{x}^{\wedge} \mathrm{d} /\left(\mathrm{b}^{\wedge} \mathrm{d}+\mathrm{x}^{\wedge} \mathrm{d}\right)\right)\end{array}$ & $5 \%$ & -6.09 & 26.0 & 14.7 & 41.2 \\
\hline
\end{tabular}

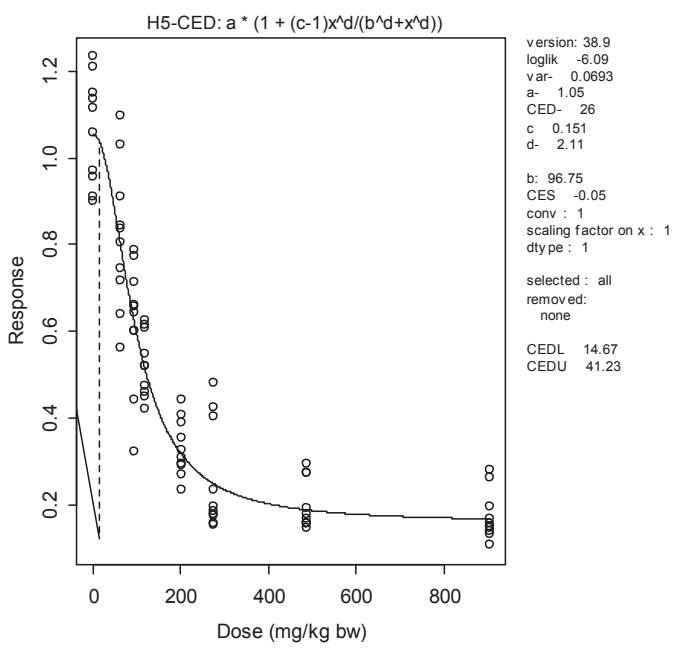

Fig. S7.1 Predicted in vivo dose-response curve for liver toxicity induced by lasiocaprine in average Chinese 
Table S7.2 Results from the BMD analysis using PROAST software of the predicted dose-response data of lasiocarpine for the average Caucasian using PBK modelling-based reverse dosimetry of cytotoxicity data derived from human hepatocytes. The table presents $\mathrm{BMD}_{5}, \mathrm{BMDL}_{5}$ and $\mathrm{BMDU}_{5}$ for a $\mathrm{BMR}$ of $5 \%$ compared to the control group, with characteristics of the model fit for Hill 5.

\begin{tabular}{|l|l|l|l|l|l|}
\hline Model & BMR & Log-likelihood & $\begin{array}{l}\text { BMD }_{5} \\
(\mathrm{mg} / \mathrm{kg} \\
\text { bw })\end{array}$ & $\begin{array}{l}\mathrm{BMDL}_{5} \\
(\mathrm{mg} / \mathrm{kg} \text { bw })\end{array}$ & $\begin{array}{l}\mathrm{BMDU}_{5} \\
(\mathrm{mg} / \mathrm{kg} \text { bw })\end{array}$ \\
\hline Full & $5 \%$ & -5.7 & NA & NA & NA \\
\hline $\begin{array}{l}\text { Hill 5-CED } \\
\mathrm{a}^{*}\left(1+(\mathrm{c}-1) \mathrm{x}^{\wedge} \mathrm{d} /\left(\mathrm{b}^{\wedge} \mathrm{d}+\mathrm{x}^{\wedge} \mathrm{d}\right)\right)\end{array}$ & $5 \%$ & -7.37 & 14.1 & 7.4 & 23.7 \\
\hline
\end{tabular}

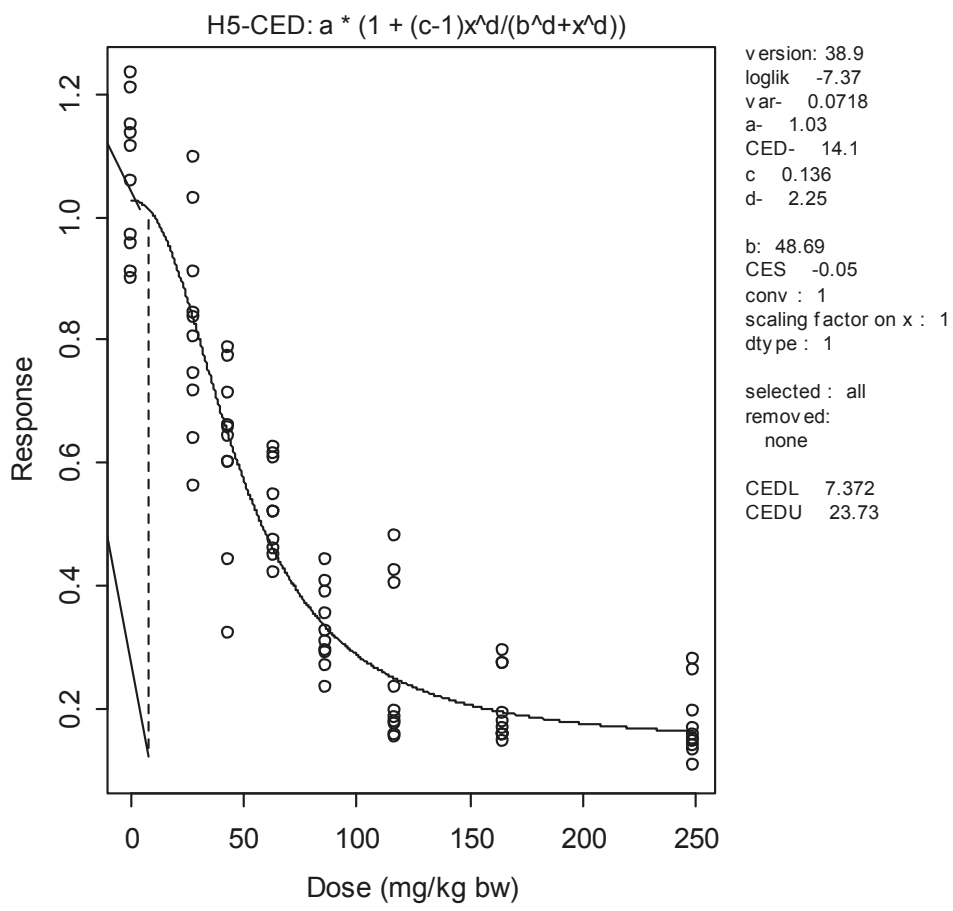

Fig. S7.2 Predicted in vivo dose-response curve for liver toxicity induced by lasiocaprine in average Caucasian 
Table S7.3 Results from the BMD analysis using PROAST software of the predicted dose-response data of riddelliine for the average Chinese using PBK modelling-based reverse dosimetry of cytotoxicity data derived from human hepatocytes. The table presents the $\mathrm{BMD}_{5}, \mathrm{BMDL}_{5}$ and $\mathrm{BMDU}_{5}$ for a $\mathrm{BMR}$ of $5 \%$ compared to the control group, with characteristics of the model fit for Hill 5.

\begin{tabular}{|l|l|l|l|l|l|}
\hline Model & BMR & Log-likelihood & $\begin{array}{l}\text { BMD }_{5} \\
(\mathrm{mg} / \mathrm{kg} \text { bw })\end{array}$ & $\begin{array}{l}\text { BMDL } \\
(\mathrm{mg} / \mathrm{kg} \text { bw })\end{array}$ & $\begin{array}{l}\text { BMDU }_{5} \\
(\mathrm{mg} / \mathrm{kg} \text { bw })\end{array}$ \\
\hline Full & $5 \%$ & 31.77 & NA & NA & NA \\
\hline $\begin{array}{l}\text { Hill 5-CED } \\
\mathrm{a}^{*}\left(1+(\mathrm{c}-1) \mathrm{x}^{\wedge} \mathrm{d} /\left(\mathrm{b}^{\wedge} \mathrm{d}+\mathrm{x}^{\wedge} \mathrm{d}\right)\right)\end{array}$ & $5 \%$ & 30.98 & 2.6 & 1.0 & 5.9 \\
\hline
\end{tabular}

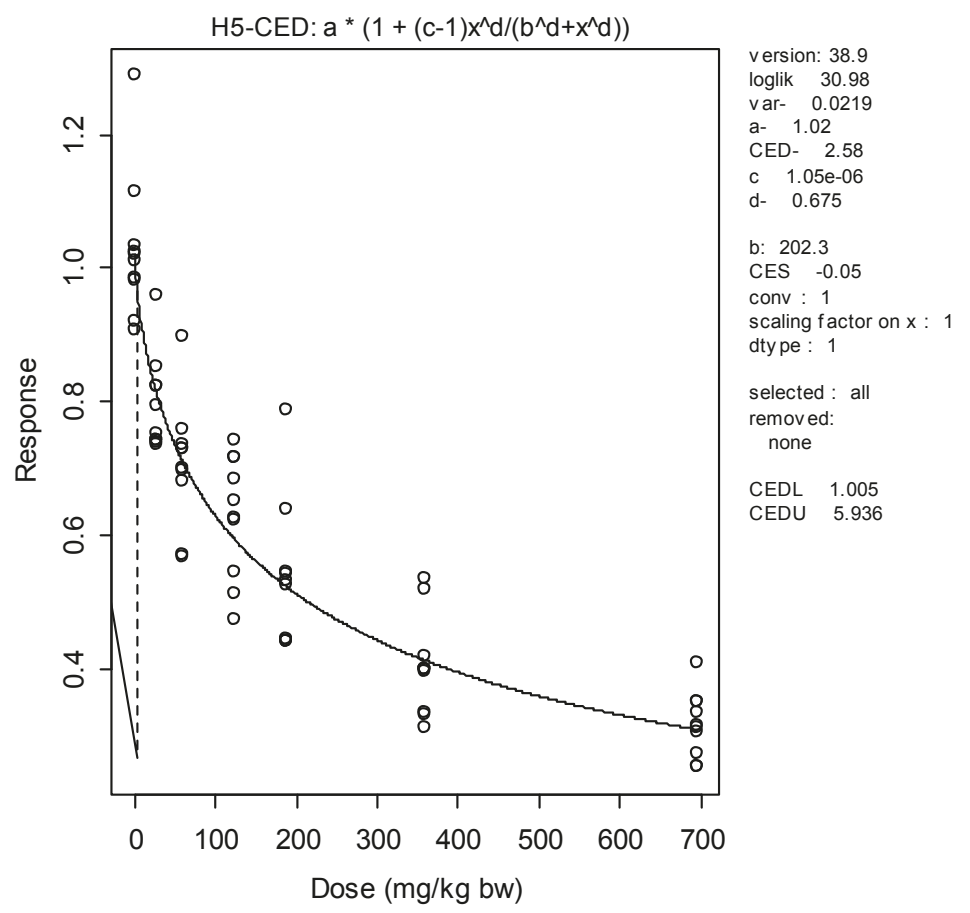

Fig. S7.3 Predicted in vivo dose-response curve for liver toxicity induced by riddelliine in average Chinese 
Table S7.4 Results from the BMD analysis using PROAST software of the predicted dose-response data of riddelliine for the average Caucasian using PBK modelling-based reverse dosimetry of cytotoxicity data derived from human hepatocytes. The table presents the $\mathrm{BMD}_{5}, \mathrm{BMDL}_{5}$ and $\mathrm{BMDU}_{5}$ for a $\mathrm{BMR}$ of $5 \%$ compared to the control group, with characteristics of the model fit for Hill 5.

\begin{tabular}{|l|l|l|l|l|l|}
\hline Model & BMR & Log-likelihood & $\begin{array}{l}\text { BMD }_{5} \\
(\mathrm{mg} / \mathrm{kg} \text { bw })\end{array}$ & $\begin{array}{l}\text { BMDL5 } \\
(\mathrm{mg} / \mathrm{kg} \text { bw })\end{array}$ & $\begin{array}{l}\mathrm{BMDU}_{5} \\
(\mathrm{mg} / \mathrm{kg} \text { bw })\end{array}$ \\
\hline Full & $5 \%$ & 31.77 & NA & NA & NA \\
\hline $\begin{array}{l}\text { Hill 5-CED } \\
\mathrm{a}^{*}\left(1+(\mathrm{c}-1) \mathrm{x}^{\wedge} \mathrm{d} /\left(\mathrm{b}^{\wedge} \mathrm{d}+\mathrm{x}^{\wedge} \mathrm{d}\right)\right)\end{array}$ & $5 \%$ & 31.25 & 0.5 & 0.2 & 1.2 \\
\hline
\end{tabular}

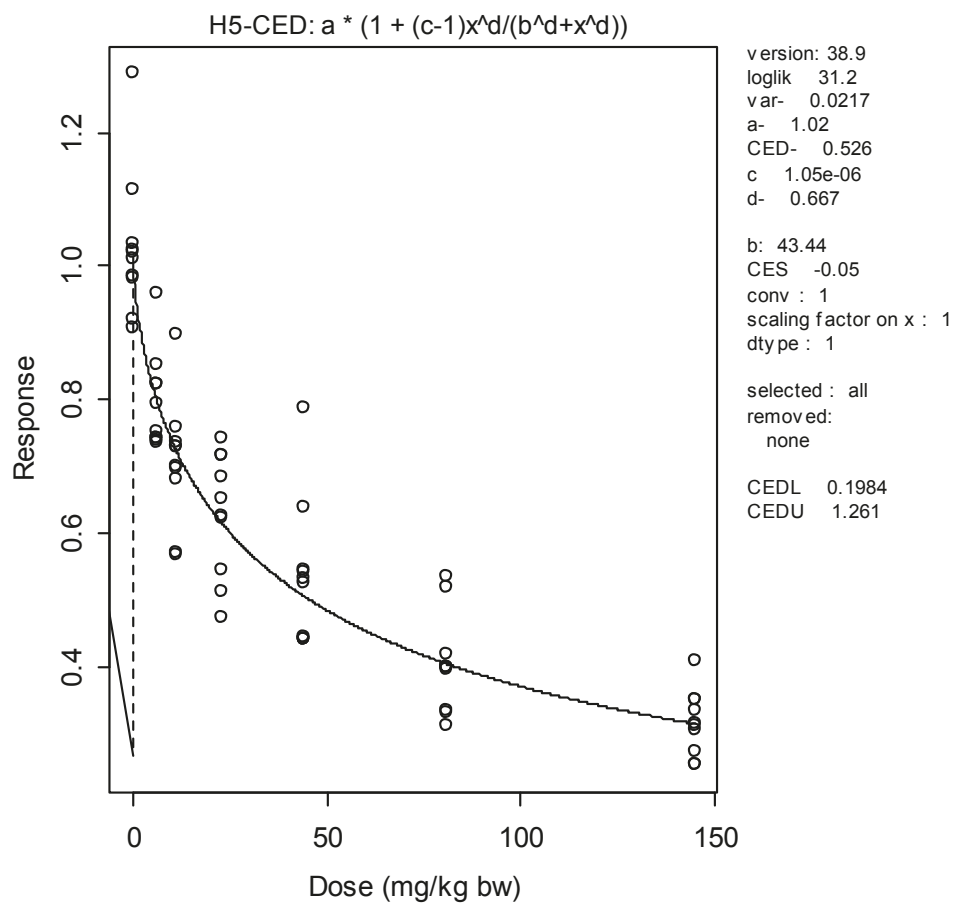

Fig. S7.4 Predicted in vivo dose-response curve for liver toxicity induced by riddelliine in average Caucasian 



\section{Chapter 6}

Prediction of in vivo genotoxicity of lasiocarpine and riddelliine in rat liver using a combined in vitro-physiologically based kinetic modelling-facilitated reverse dosimetry approach

Lu Chen, Ad Peijnenburg, Laura de Haan, Ivonne M.C.M. Rietjens

Published in: Archives of Toxicology, (2019), 1-11 


\begin{abstract}
Pyrrolizidine alkaloids (PAs) are naturally occuring genotoxic compounds, and PA-contaning plants can pose a risk to humans through contaminated food sources and herbal products. Upon metabolic activation, PAs can form DNA adducts, DNA and protein cross-links, chromosomal aberrations, micronuclei and DNA double-strand breaks. These genotoxic effects may induce gene mutations and play a role in the carcinogenesis of PAs. This study aims to predict in vivo genotoxicity for two wellstudied PAs, lasiocarpine and riddelliine, in rat using in vitro genotoxicity data and physiologically based kinetic (PBK) modelling-based reverse dosimetry. The $\gamma \mathrm{H} 2 \mathrm{AX}$ assay was used to determine the in vitro genotoxicity for lasiocarpine and riddelliine in primary rat hepatocytes and human HepaRG cells. The in vitro concentration-response curves obtained from primary rat hepatocytes were subsequently converted to in vivo dose-response curves from which points of depature (PoDs) were derived that were compared to available in vivo genotoxicity data. The results showed that the predicted PoDs for lasiocarpine and riddelliine were comparable to in vivo genotoxicity data. It is concluded that this quantitative in vitro-in silico approach provides a method to predict in vivo genotoxicity for the large number of PAs for which in vivo genotoxicity data are lacking by integrating in vitro genotoxicity assays with PBK modelling-facilitated reverse dosimetry.
\end{abstract}




\section{Introduction}

Pyrrolizidine alkaloids (PAs) are secondary metabolites that plants produce against insects (Fu et al., 2004). To date, more than 660 PAs and their N-oxides have been identified from more than 6000 plants, and half of them are hepatotoxic (Fu et al., 2010; Wiedenfeld, 2011). PA containing plants are widely distributed around the world, and a number of human and livestock poisoning incidents with high mortality due to (unintentional) contamination by PAs have been reported in different countries (Creeper et al., 1999; Edgar et al., 2014; Hill et al., 1997; Molyneux et al., 2011). Human exposure to PAs mainly results from PA containing foods, such as herbal teas, herbal medicines, milk and honey (Bodi et al., 2014; EFSA, 2016, EFSA 2017; Kempf et al., 2010; Mathon et al., 2014; Roeder, 2000). PAs can induce acute hepatotoxicity in both rat and human, resulting in liver necrosis, hepatomegaly, and veno-occlusive disease (VOD) (Fu et al., 2007; Fu et al., 2004; Lin et al., 2011; Mattocks, 1986; Wiedenfeld, 2011). Chronic exposure to PAs is of concern because of their genotoxicity and carcinogenicity (EFSA, 2011). Especially 1,2-unsaturated PAs are hepatotoxic and considered to be genotoxic carcinogens, thus posing a potential risk to human health (Mori et al., 1985).

Upon metabolic activation PAs can lead to a variety of genotoxic effects, including formation of DNA adducts, DNA and protein cross-links, chromosomal aberrations, micronuclei and DNA double-strand breaks (DSB) (Allemang et al., 2018; Chou et al., 2003; Chou et al., 2004; Fu et al., 2001; Fu et al., 2010; Fu et al., 2004; Prakash et al., 1999; Uhl et al., 2000; Wang et al., 2005a; Xia et al., 2006; Xia et al., 2013). These genotoxic effects may result in gene mutations and tumour formation (Chen et al., 2010; Hoeijmakers, 2009). Recently, the European Food Safety Authority (EFSA) has drawn attention to the fact that more data on the toxicokinetics and genotoxicity of individual PAs would greatly refine their risk assessment (EFSA, 2017). State-of-the-art genotoxicity and carcinogenicity data on PAs are limited, and among the 1,2-unsaturated PAs, lasiocarpine and riddelliine are the PAs most studied in in vivo genotoxicity and carcinogenicity studies. For instance, both PAs have been shown to induce genotoxicity in rat liver upon oral exposure (Chan et al., 1994; Xia et al., 2013; Yan et al., 2002). In addition, they were both tested in oral 2-year carcinogenicity studies and shown to induce liver haemangiosarcomas in rats upon chronic oral exposure (NTP, 1978, 2003). In addition, limited in vivo genotoxicity data are available for seneciphylline, senkirkine, monocrotaline and retrorsine (Candrian et al., 1984; Wang et al., 2005a; Wang et al., 2005b), and results from carcinogenicity bioassays have been reported for monocrotaline, clivorine, petasitenine, senkirkine and symphytine with only in some of these studies rats being dosed via the oral route (Hirono et al., 1979; Hirono et al., 1976; Kuhara et al., 1980; Shumaker et al., 1976). Studies to assess whether exposure to PAs results in genotoxicity and carcinogenicity in humans are not available. Lack of genotoxicity studies for most 1,2-unsaturated PAs, hampers PA risk assessment, while economic and ethical constraints limit the possibilities to perform in vivo genotoxicity and carcinogenicity studies for all relevant PAs, including 
the 17 PAs identified by EFSA to be relevant for exposure via feed and food (EFSA, 2017). Thus, alternative testing strategies for estimating the genotoxicity and carcinogencity of different PAs are required.

In our previous studies, we have successfully predicted in vivo acute liver toxicity for lasiocarpine and riddelliine in rat using physiologically based kinetic (PBK) modelling-facilitated reverse dosimetry (Chen et al., 2018). In this approach, in vitro concentration-response curves for cytotoxicity of lasiocarpine and riddelline in primary rat hepatocytes were translated to in vivo dose-response curves for acute liver toxicity in rat using PBK modelling (Chen et al., 2018). The aim of the present study was to investigate whether the developed PBK modelling-facilitated reverse dosimetry approach can also adequately translate in vitro concentration-response curves for genotoxicity of lasiocarpine and riddelliine, to in vivo dose-response curves for genotoxicity of these PAs. To this end, the in vitro concentration-responses curves obtained from in vitro genotoxicity studies with primary rat hepatocytes using the $\gamma \mathrm{H} 2 \mathrm{AX}$ in-cell Western (ICW) assay were translated into in vivo dose-response curves by using PBK modelling-facilitated reverse dosimetry. The $\gamma \mathrm{H} 2 \mathrm{AX}$ ICW assay allows to quantify the amount of phosphorylated histone $\mathrm{H} 2 \mathrm{AX}(\gamma \mathrm{H} 2 \mathrm{AX})$, which is known to be associated with DNA damage (Audebert et al., 2010; Khoury et al., 2013). Recently, this assay has been successfully applied to determine the in vitro genotoxic potencies of a large number of PAs, belonging to different chemical classes, in the human liver cell line HepaRG (Louisse et al., Submitted). In the present study, the predicted in vivo dose-response curves were used to determine the points of departure (PoDs) which were compared to available in vivo data for genotoxicity induced by lasiocarpine and riddelliine in rats.

\section{Materials and methods}

\section{Chemicals}

Lasiocarpine (> 97\%) was purchased from PhytoLab (PhytoLab GmbH \& Co. KG, Germany) (primary rat hepatocyte studies and HepaRG studies), riddelliine (95.4\%) was kindly provided by the National Toxicology Program (NTP) (U.S. Department of Health and Human Service) (primary rat hepatocyte studies) and from Dr. Tao Chen (U.S. FDA) as a generous gift (HepaRG studies). Corning 96 Well cellbind black microplates were obtained from Sigma-Aldrich (St. Louis, MO, USA). The cryopreserved rat (Sprague-Dawley) hepatocytes, thawing/plating supplement pack, cell maintenance supplement pack, fetal bovine serum (FBS) and Williams E Medium (A1217601) were purchased from ThermoFisher (Naarden, The Netherlands). HepaRG cells were obtained from Biopredic International (Saint-Grégoire, France). William's E medium and glutamax were obtained from Life Technologies (Paisley, UK). Primary antibody 'anti-Phospho Histone $\mathrm{H}_{2} \mathrm{AX}$ ' (ser 139) (20E3) rabbit $\mathrm{mAb}$, and secondary antibody CF770 goat anti rabbit $\mathrm{IgG}(\mathrm{H}+\mathrm{L})$ were purchased from Cell Signaling Technology (Danvers, MA, USA) and Biotium (Fremont, CA, USA), respectively. Fetal bovine serum (FBS) and human insulin were purchased from PanBiotech (Aidenbach, Germany). 
Penicillin/Streptomycin was from Capricorn Scientific (Ebsdorfergrund, Germany). Phosphate buffered saline (PBS) was obtained from Lonza BioWhittaker (Walkersville, MD, USA). Dimethyl sulfoxide (DMSO) was obtained from Acros Organics (Geel, Belgium). Acetonitrile (UPLC/MS grade) was obtained from Biosolve (Valkenswaard, The Netherlands). Triton X-100, bovine pancrease RNase A was purchased from Sigma-Aldrich (St Louis). PhosStop tablet was purchased from Roche Diagnostics (Mannheim, Germany). 16\% Paraformaldehyde (PFA) was purchased from Electron microscopy sciences (Hatfield, USA). Reddot was obtained from Biotium (Fremont) and MaxBlock was purchased from Active Motif (Carlsbad, CA, USA).

\section{Cell culture}

Two types of cells were used for determing in vitro concentration-response curves for genotoxicity, i.e. primary rat hepatocytes and HepaRG cells. Briefly, cryopreserved primary rat hepatocytes were seeded at concentration of $5 \times 10^{5}$ cells/well in black 96 well cellbind plates, and incubated in Williams' Medium E (no phenol red) consisting of hepatocyte plating supplement pack (CM3000, serumcontaning) for 4-6 hours before exposure. Only batches with a cell viability $>90 \%$ were used for experiments. HepaRG cells were grown in growth medium consisting of Williams' medium E, supplemented with 10\% FBS, $100 \mathrm{IU} / \mathrm{ml}$ penicillin, $100 \mu \mathrm{g} / \mathrm{ml}$ streptomycin, 1\% (v/v) L-glutamine, 5 $\mu \mathrm{g} / \mathrm{ml}$ human insulin and $50 \mu \mathrm{M}$ hydrocortisone-21-hemisuccinate. The growth medium was renewed every two to three days. After two weeks, the HepaRG cells were trypsinized and seeded at a concentration of $9 \times 10^{3}$ cells/well in black 96 well cellbind plates, and kept on growth medium for two weeks and subsequently for another two weeks on differentiation medium consisting of growth medium supplemented with $1.7 \%$ (v/v) DMSO (Aninat et al. 2006). The medium was refreshed every two to three days. After two weeks culturing in differentiation medium, HepaRG cells were fully differentiated and used for experiments within four weeks with refreshing the medium every two to three days according to the protocol provided by Biopredic International. Primary rat hepatocytes and HepaRG cell were incubated in a 5\% $\mathrm{CO}_{2}: 95 \%$ air-humidified incubator.

\section{$\gamma \mathrm{H} 2 \mathrm{AX}$ assay}

The $\gamma \mathrm{H} 2 \mathrm{AX}$ ICW assay allows to simultaneously determine genotoxicity and cytotoxicity (Audebert et al., 2010; Khoury et al., 2013) and was used in the present study to assess the in vitro genotoxicity of non-cytotoxic concentrations of lasiocarpine and riddelliine in both primary rat hepatocytess and HepaRG cells. 4-6 hours after seeding the rat hepatocytes, the medium was replaced by exposure medium (Williams' Medium E no phenol red supplemented with hepatocyte plating supplement pack (CM4000, serum-free)), containing lasiocarpine (final concentrations: 0 (solvent control), 0.006, 0.012, $0.024,0.048,0.09,0.195,0.39,0.78,1.56,3.125$ and $6.25 \mu \mathrm{M}$ ) or riddelliine (final concentrations: 0 (solvent control), 0.065, 0.13, 0.26, 0.52, 1.04, 2.08, 4.16, 8.32, 10 and $15 \mu \mathrm{M}$ ). Each concentration was tested in three replicates, and two independent experiments with different batches of primary rat hepatocytes were carried out. The HepaRG cells were placed on growth medium supplemented with 
$0.5 \%$ DMSO for 24 hours before exposure to the compounds. After 24 hours treatment, the HepaRG cells were treated with exposure medium (growth medium without FBS) contaning lasiocarpine (final concentrations: 0 (solvent control), 0.78, 1.56, 3.13, 6.25, 12.5, 25 and $50 \mu \mathrm{M}$ ) or riddelliine (final concentrations: 0 (solvent control), 1.56, 3.13, 6.25, 12.5, 25 and $50 \mu \mathrm{M}$ ) in duplicates with three independent experiments. Lasiocarpine and riddelliine used in the $\gamma \mathrm{H} 2 \mathrm{AX}$ assay were added as 200 times concentrated stock solutions in DMSO, solvent controls were treated with DMSO (final concentration: $0.5 \%)$.

The $\gamma \mathrm{H} 2 \mathrm{AX}$ in-cell western (ICW) technique was performed essentially as previously described (Audebert et al., 2010; Khoury et al., 2013). Briefly, primary rat hepatocytes and HepaRG cells were treated with lasiocarpine and riddelliine for 24 hours, after 24 hours treatment the medium was removed and the cells were washed with PBS. After washing, the cells were fixed with 4\% PFA in PBS at room temperature, and subsequently washed with PBS. PFA was neutralized with $20 \mathrm{mM}$ $\mathrm{NH}_{4} \mathrm{Cl}$ and the wells were washed with PBS. Cells were permeabilized with $0.2 \%$ Triton X-100 in PBS and washed with PST buffer (PBS containg 2\% FBS and 0.2\% Triton X-100). Cells were blocked with MAXblock Blocking medium supplemented with phosphatase inhibitor PHOSTOP and RNase A, followed by $2 \mathrm{~h}$ incubation with rabbit monoclonal anti $\gamma \mathrm{H} 2 \mathrm{AX}$ in PST buffer. After three washes with PST, secondary detection was carried out using an infrared fluorescent dye conjugated to goat antibody with an absorption peak at $770 \mathrm{~nm}$ in PST buffer. For DNA labelling, RedDot in PST was used together with the secondary antibody. The RedDot signal is used as a measure for cell number, allowing normalization of the $\gamma \mathrm{H} 2 \mathrm{AX}$-response to cell number. Only concentrations resulting in cytotoxicity lower than $20 \%$ (i.e cell viability of at least $80 \%$ ) were used for further data evaluation. After $1 \mathrm{~h}$ of incubation and subsequent three washes with PST, RedDot and $\gamma \mathrm{H} 2 \mathrm{AX}$ signals were simultaneously visualized using an Odyssey Infrared Imaging Scanner (LiCor ScienceTec, Les Ulis, France). The quantification of phosphorylated histone $\mathrm{H} 2 \mathrm{AX}(\gamma \mathrm{H} 2 \mathrm{AX})$ is expressed relative to the amount of $\gamma \mathrm{H} 2 \mathrm{AX}$ detected in cells exposed to the vehicle control. Error bars represent SD (standard deviation) of the mean. Statistically significant increases in H2AX phosphorylation after treatment were compared with controls using $t$-test with Graphpad software.

\section{PBK modelling-based reverse dosimetry}

The PBK modelling-facilated reverse dosimetry approach to predict in vivo genotoxicity based on in vitro genotoxicity data consisted of the following steps: (1) determination of in vitro concentrationresponse curves for genotoxicity of lasiocarpine and riddelliine in primary rat hepatocytes and HepaRG cells, (2) translation of the in vitro concentration-response curves to in vivo dose-response curves for rat using previously developed PBK models (Chen et al., 2018), (3) BMD analysis on the predicted in vivo dose-response curves to derive the points of depature (PoDs), (4) evaluation of the predicted PoD values with the values obtained from in vivo genotoxicity studies. 
In the step 2, the possible differences in protein binding due to different protein levels in the medium of the in vitro assay and the in vivo situation should be considered. We previously used RED (rapid equilibrium dialysis) to detect the fraction unbound ( $\mathrm{f}_{\mathrm{ub}}$ ) of lasiocarpine and riddelliine in rat serum, resulting in $\mathrm{f}_{\mathrm{ub}}$ values amounting to 0.64 for lasiocarpine and 0.66 for riddelliine (Chen et al., 2018). Thus, the effect concentrations of lasiocarpine and riddelliine in rat blood $\left(\mathrm{C}_{\mathrm{Lc} / \mathrm{Rd}}\right.$, rat blood $)$ applied for reverse dosimetry were corrected by the following equation: $C_{L c / R d}$, rat blood $=C_{u b}$, in vitro $/ f_{u b \text {, rat blood. }}$. Where $\mathrm{C}_{\mathrm{ub}}$, in vitro is the unbound concentration of lasiocarpine and riddelliine in the in vitro medium. Since the exposure medium used in the present study for the in vitro genotoxicity asssay was serum free, the concentration of lasiocarpine and riddelliine in the in vitro $\gamma \mathrm{H} 2 \mathrm{AX}$ assay was considered to be equal to the unbound concentration in rat serum.

When considering an endpoint like genotoxicity the total dose over time and thus the area under the curve (AUC) presents the most relevant parameter for reverse dosimetry (Groothuis et al., 2015). To this end, the in vitro concentration-response data were translated to in vitro AUC-response data, by multiplying the effect concentration with the assay time (24 hours) (Daston et al. 2010). The AUCresponse curves thus obtained were translated to the in vivo situation setting them equal to the unbound AUC values in the plasma.

\section{BMD analysis of in vitro concentration-response curves and of predicted in vivo dose- response data}

In order to support the validity and use of the in vitro-in silico approach the predicted $\mathrm{BMD}_{10}$ (the benchmark dose responsible for a $10 \%$ response) values for lasiocarpine and riddelliine were compared with PoDs derived from in vivo genotoxicity data from the literature (Chan et al., 1994; Xia et al., 2013; Yan et al., 2002). The continuous model using summary data from the PROAST software (version 66.42, the Dutch National Insitude for Public Health and the Environment, The Netherlands) was applied to analyse the predicted in vivo dose-response data for lasiocarpine and riddelliine and to derive the $\mathrm{BMD}_{10}$ values. The goodness of fit was used to judge if the model could be accepted. Only a model fit with $\mathrm{P}>0.05$ was considered acceptable for the determination of $\mathrm{BMD}_{10}$ values.

\section{Results}

\section{In vitro genotoxicity}

Figure $1 \mathrm{a}$ and $\mathrm{b}$ present the in vitro concentration-response curves for genotoxicity of lasiocarpine and riddelliine in the $\gamma \mathrm{H} 2 \mathrm{AX}$ assay in primary rat hepatocytes and metabolic competent HepaRG cells, respectively. The PROAST program was applied to analyse the concentration-response data and to determine the critical effect concentration, i.e. $\mathrm{BMC}_{10}$ (the benchmark concentration responsible for a $10 \%$ response). Histone $\mathrm{H} 2 \mathrm{AX}$ phosphorylation in these two types of cells increased upon exposure to lasiocarpine and riddelliine in a dose-dependent manner (Figure 1). Table 1 shows the $\mathrm{BMC}_{10}$ values of lasiocarpine and riddelliine derived from these concentration-response curves. The primary rat 
hepatocytes appear more sensitive towards the genotoxicity of lasiocarpine and riddelliiine than the HepaRG cells, since the $\mathrm{BMC}_{10}$ values for genotoxicity in the $\gamma \mathrm{H} 2 \mathrm{AX}$ assay for lasiocarpine and riddelliine derived from primary rat hepatocytes were respectively 105- and 31-fold lower than those obtained in the HepaRG cells.

Table $1 \mathrm{BMC}_{10}$ values for lasiocarpine and riddelliine derived from the concentration-response curves presented in Figure 1

\begin{tabular}{ll}
\hline Cell model & $\mathrm{BMC}_{10}(\mu \mathrm{M})$ \\
\hline Primary rat hepatocytes & \\
Lasiocarpine & 0.02 \\
Riddelliine & 0.07 \\
HepaRG cells & \\
Lasiocarpine & 2.09 \\
Riddelliine & 2.17 \\
\hline
\end{tabular}
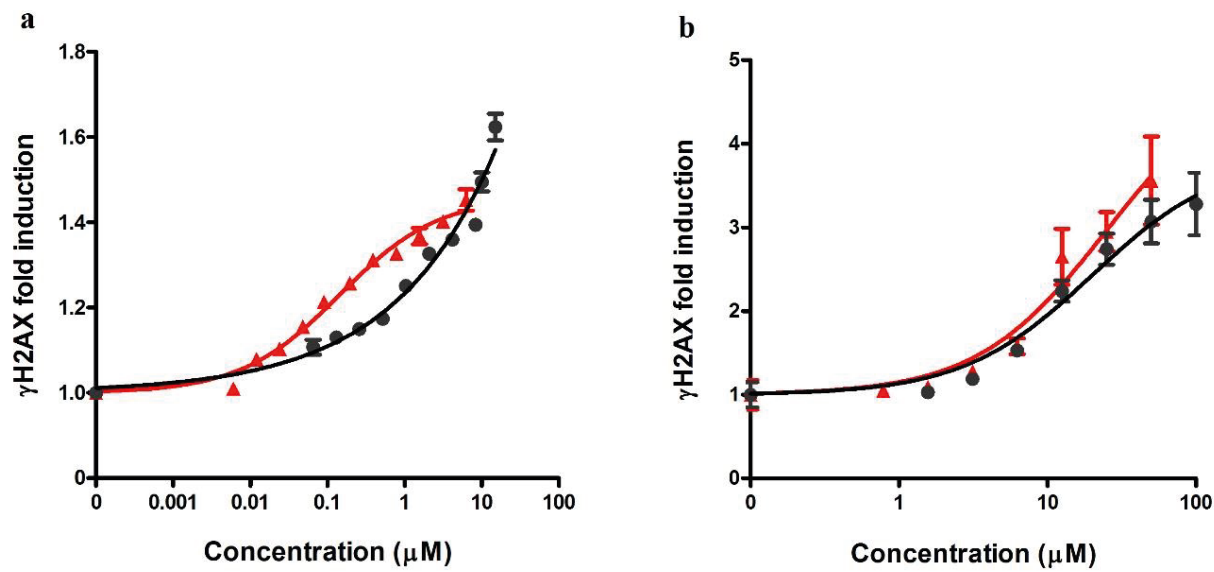

Fig. 1 Concentration-response curves for $\gamma \mathrm{H} 2 \mathrm{AX}$ induction in primary rat hepatocytes (a) and HepaRG cells (b) treated with increasing concentration of lasiocarpine (red triangle) and riddelliine (black circle) for 24 hours $($ mean $\pm \mathrm{SD})$

\section{Translation of the in vitro concentration-response curves to the in vivo dose-response curves and prediction of in vivo BMD10 values}

The in vitro concentration-response curves obtained for primary rat hepatocytes were first corrected for differences in binding to proteins in medium (in vitro) and serum (in vivo), resulting in effect concentrations, which were then converted to AUC-response curves by multiplying the effect concentration with 24 hours, the duration of the in vitro incubations. Figure 2 a presents the AUCresponse curves thus obtained. These in vitro AUC-response curves were subsequently translated into predicted dose-response curves using the respective PBK models (Figure 2b). The dose levels thus 
obtained for the dose-response curves are shown in the Supplementary data (Table 1). From these predicted dose-response curves, the $\mathrm{BMD}_{10}$ values for lasiocarpine and riddelliine were derived amounting to 8.82 and $3.41 \mathrm{mg} / \mathrm{kg}$ bw/day, respectively (Table 2). Compared to the in vitro genotoxicity data in primary rat hepatocytes where lasiocarpine was 3.5-fold more active than riddelliine (Figure 1a and 2a), the predicted in vivo genotoxicity of riddelliine appeared to be 2.6-fold higher than that of lasiocarpine (Figure 2b). This can be ascribed to the differences in kinetics with a slower clearance of riddelliine compared to lasiocarpine, with the total scaled in vivo catalytic efficiency in rat for depletion of riddelliine previously shown to be 11-fold lower than that for lasiocarpine (Chen et al., 2018).

Table 2 Predicted $\mathrm{BMD}_{10}$ values for lasiocarpine and riddelliine obtained using PBK modelling-facilitated reverse dosimetry, which allowed translation of the in vitro AUC-genotoxicity curves (Figure 2a) to the in vivo dose-response curves (Figure 2b)

Compound Predicted $\mathrm{BMD}_{10}(\mathrm{mg} / \mathrm{kg}$ bw/day $)$

Lasiocarpine $\quad 8.82$

Riddelliine $\quad 3.41$

a

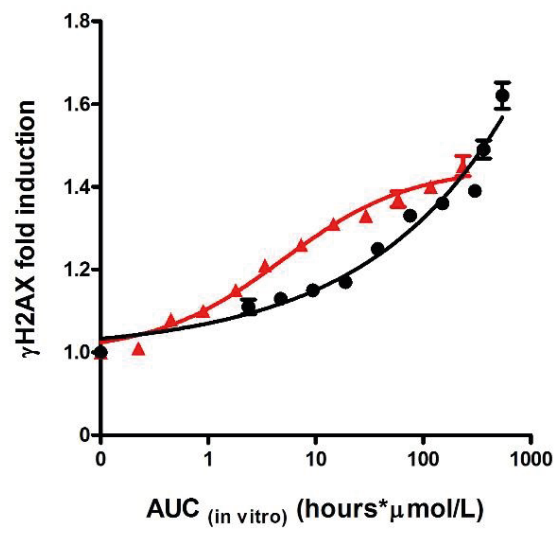

b

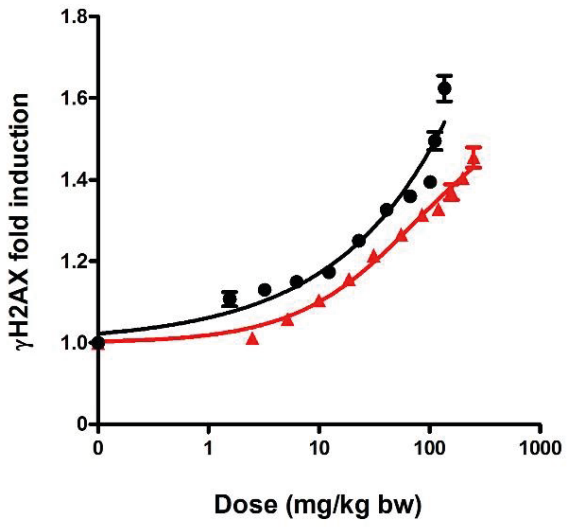

Fig. 2 The in vitro AUC-response curves for lasiocarpine and riddelliine obtained with primary rat hepatocytes using the $\gamma \mathrm{H} 2 \mathrm{AX}$ assay (a) and in vivo dose-response curves for genotoxicity in rat liver predicted from in vitro AUC-response curves using PBK modelling (b). Lasiocarpine (red triangle); riddelliine (black circle). Data expressed as (mean $\pm \mathrm{SD}$ ) 


\section{Evaluation of the predicted PoDs for in vivo genotoxicity}

To evaluate the outcomes of the in vitro-PBK model based predictions for dose-dependent genotoxicity of lasicoarpine and riddelliine in rat liver, the predicted $\mathrm{BMD}_{10}$ values for lasiocarpine and riddelliine were compared to the in vivo genotoxicity data in rat liver that have been published previously (Chan et al., 1994; Xia et al., 2013; Yan et al., 2002). Table 3 presents an overview of literature data in rat hepatocytes in vivo, measuring endpoints including DNA adduct formation and unscheduled DNA synthesis. The data from these in vivo animal studies were not suitable for BMD analysis due to the limited number of data points and insufficient distribution of the data points over the dose-response curves. Therefore, the lowest observed adverse effect level (LOAEL) of lasiocarpine and riddelliine that induced in vivo genotoxicity were used for the validation (Table 3 ). Figure $3 \mathrm{a}$ and $\mathrm{b}$ shows a comparison of the predicted $\mathrm{BMD}_{10}$ values for in vivo genotoxicity of lasiocarpine and riddelliine and these LOAEL values derived from in vivo literature rat data for genotoxicity of lasiocarpine and riddelliine. For lasiocarpine, the predicted $\mathrm{BMD}_{10}$ was only 1.1-fold different (lower) compared to the LOAEL derived from the in vivo study detecting DNA adduct formation (Xia et al., 2013) (Figure 3a), while it was higher than the dose level of $1.85 \mathrm{mg} / \mathrm{kg}$ bw at which lasiocarpine derived DNA adducts were not yet detectable. At this dose level riddelliine DNA adducts could already be detected in the in vivo study, which is in line with the predicted $\mathrm{BMD}_{10}$ values which indicated riddelliine to have a lower $\mathrm{BMD}_{10}$ value than lasiocarpine. For riddelliine, the predicted $\mathrm{BMD}_{10}$ was only 1.8-fold different (higher) compared to the LOAEL derived from in vivo experimental data measuring DNA-adduct formation (Xia et al., 2013). Comparison to the dose level inducing detectable DNA adduct formation by riddeliine of $10 \mathrm{mg} / \mathrm{kg}$ bw/day in the study reported by Yan et al. (Yan et al., 2002), showed that the prediction is 2.9-fold lower, but this may in part be due to the fact that the effective dose level reported in this study was the only dose level tested, and dose levels lower than $10 \mathrm{mg} / \mathrm{kg}$ bw/day were not included in the study. Compared to the lowest dose level that causes a statistically significant increase in unscheduled DNA synthesis the predicted $\mathrm{BMD}_{10}$ for riddelliine was 3.4-fold higher (Chan et al., 1994). 


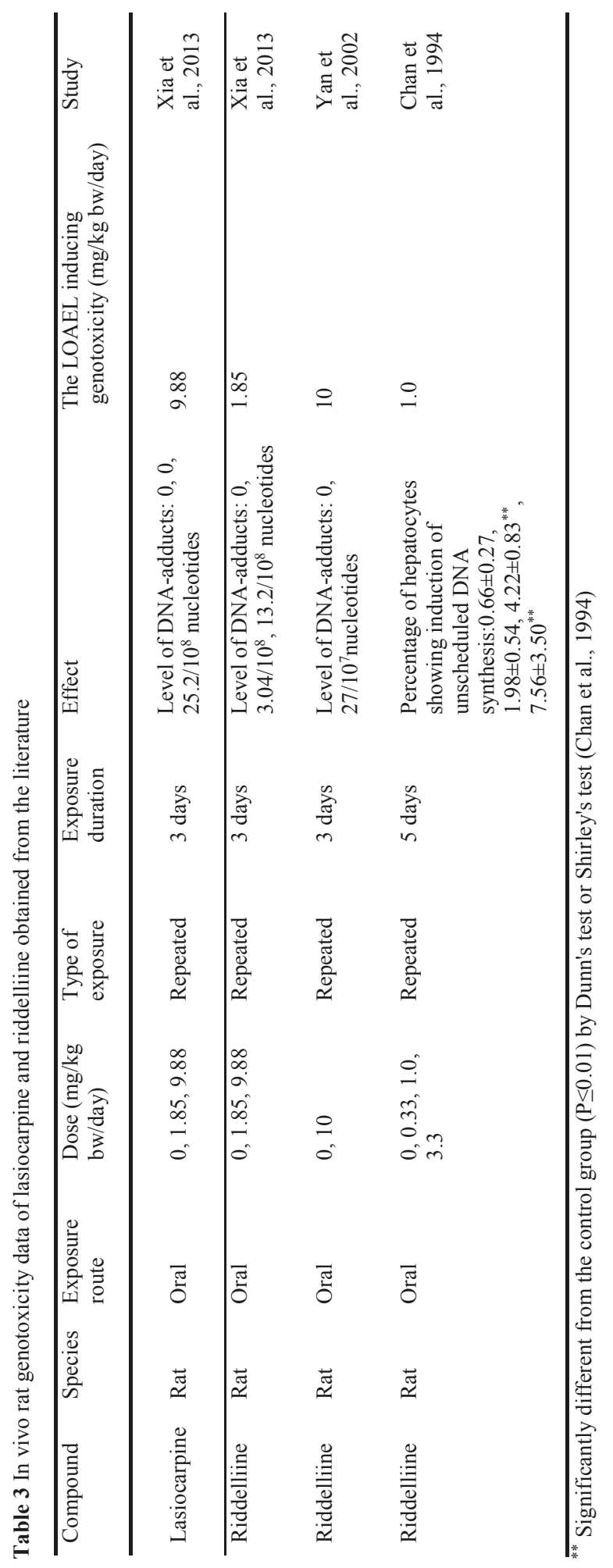




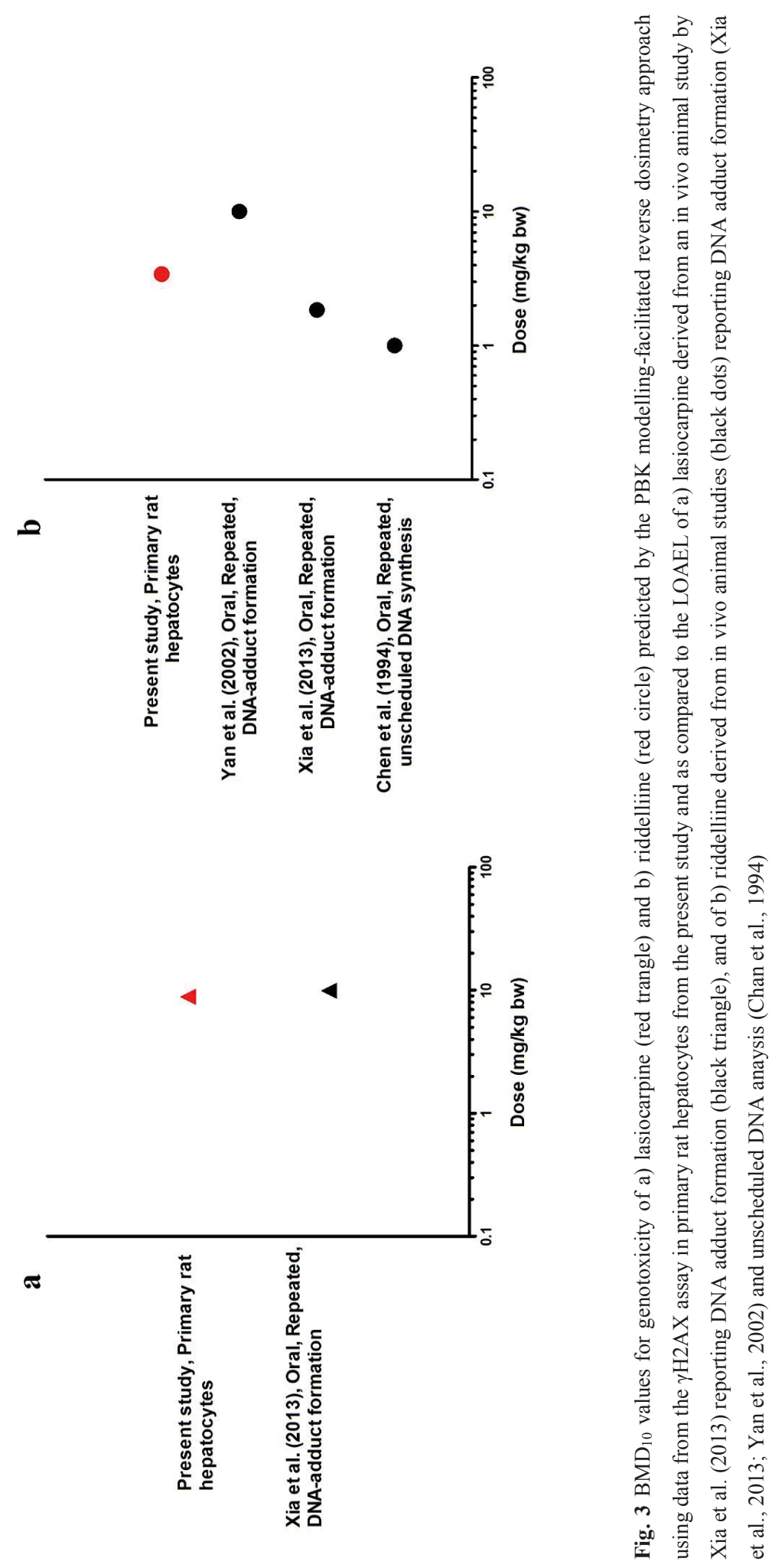




\section{Discussion}

The aim of present study was to assess whether the developed PBK modelling-facilitated reverse dosimetry could adequately predict in vivo dose-response curves for genotoxicity of lasiocarpine and riddelliine in rats. The $\gamma \mathrm{H} 2 \mathrm{AX}$ ICW assay was used to quantify the genotoxicity of these PAs in primary rat hepatocytes. So far, the $\gamma \mathrm{H} 2 \mathrm{AX}$ ICW assay has been successfully applied to evaluate the genotoxiciy of several food contaminants, including aflatoxins (Theumer et al., 2018), heavy metals (Kopp et al. 2018), polycyclic aromatic hydrocarbons (Audebert et al., 2010; Audebert et al., 2012) and PAs (Louisse et al., Submitted), for possible genotoxicity.

To date, lasiocarpine and riddelliine have been reported to induce genotoxic effects in in vitro assays for different endpoints including formation of micronuclei (Allemang et al., 2018), DNA-adducts (Xia et al., 2006), chromosomal aberrations (Takanashi et al., 1980), DNA-cross links (Kim et al., 1995) and sister chromatid exchange (Chan et al., 1994). Comparison of the results obtained in the present study using the $\gamma \mathrm{H} 2 \mathrm{AX}$ assay to these published genotoxic data, reveals that also the phosphorylation of histone $\mathrm{H} 2 \mathrm{AX}(\gamma \mathrm{H} 2 \mathrm{AX})$ provides a relevant readout for determining in vitro genotoxicity of PAs. $\gamma \mathrm{H} 2 \mathrm{AX}$ has been reported to correlate strongly with DNA damage and is a well-recognized precancerous biomarker in vivo, and this biomarker is present in all cell types (Bonner et al., 2008; Singh et al., 2011). One of the main advantages of the $\gamma \mathrm{H} 2 \mathrm{AX}$ assay is that it allows the simultaneous determination of genotoxicity and cytotoxicity (Graillot et al., 2012). In the present study, the cut-off value for cytotoxicity was set to $80 \%$ viable cells to exclude any false-positive genotoxic results, since $\mathrm{H} 2 \mathrm{AX}$ phosphorylation could be linked to apoptosis or necrosis rather than to true genotoxicity (Imreh et al., 2011). The cut of value of $80 \%$ selected in the present study was in line with previous studies (Clarke et al., 2012; O'donovan, 2012). Regarding metabolic activation, the primary rat hepatocytes and HepaRG cells used in the present study are considered to be relevant, because they are expect to present relevant biotransformation. As for genotoxicity, the $\gamma \mathrm{H} 2 \mathrm{AX}$ is considered to be a relevant read-out for PAs, since PAs have been shown to induce genotoxicity by different modes of actions (Chen et al., 2010), and $\gamma \mathrm{H} 2 \mathrm{AX}$ occurs after a DNA double strand break in a cell can reflect and reflects a global genotoxic insult that may originate from different types of DNA damage: DNA adduct formation, DNA single-strand breaks, DNA replication or transcription blocking lesions (Sedelnikova et al., 2010). The $\gamma \mathrm{H} 2 \mathrm{AX}$ ICW assay has been reported to detect genotoxins with such a variety of DNA damaging characteristics (Khoury et al., 2013).

In the present study both primary rat hepatocytes and HepaRG cells were used to perform the $\gamma \mathrm{H} 2 \mathrm{AX}$ assay, since these two types of cells contain the CYP450 enzymes required for biotransformation of lasiocarpine and riddelliine into DNA reactive intermediates (Fu et al., 2004). HepaRG cells are derived from a human hepatocellular carcinoma, and express most of the CYP450 enzymes, especially the CYP 3A and 2B isoforms, that are key enzymes for PAs bioactivation (Fu et al., 2004; Guillouz 
et al., 2007; Lin et al., 2003; Prakash et al., 1999; Turpeinen et al., 2009). Although the levels of these two enzymes in primary rat hepatocytes and HepaRG cells have not been quantified, our previous study, in which we assessed cytotoxicity of lasiocarpine and riddelline in both cell models, already confirmed that both cell lines contain the CYPs required for bioactivation of these PAs. The IC50 for cytotoxicity of lasiocarpine and riddelliine in primary rat hepatocytes was 20- and 22-fold lower than the IC50 in HepaRG cells, respectively (Ning et al., 2019). This indicated that HepaRG cells are capable of bioactivating PAs but likely to an about 20 -fold lower level than primary rat hepatocytes. This is why also in the present genotoxicity study we included both cell models. Quantifying $\gamma \mathrm{H} 2 \mathrm{AX}$ formation as the endpoint for genotoxicity, the primary rat hepatocytes appeared to be about one to two orders of magnitude more sensitive to lasiocarpine and riddelliine induced genotoxicity than the HepaRG cells. Allemang et al. (2018) investigated genotoxicity of lasiocarpine and riddelliine in HepaRG cells using the micronucleus assay, showing dose-dependent increases in micronucleus induction. The $\mathrm{BMC}_{10}$ values for lasiocarpine and riddelliine derived from these concentrationresponse curves amounted to 0.13 and $0.63 \mu \mathrm{M}$, respectively (Allemang et al., 2018). Comparison of these values to the $\mathrm{BMC}_{10}$ values derived from the HepRG cells in the $\gamma \mathrm{H} 2 \mathrm{AX}$ assay in the present study (Table 1) reveals that the micronucleus endpoint is 16.1- and 3.4-fold more sensitive for lasiocarpine and riddelliine, respectively. Compared to the results obtained in the present study using the primary hepatocytes in the $\gamma \mathrm{H} 2 \mathrm{AX}$ assay, the HepaRG cells in the micronucleus assay were still 6.5- and 9.0-fold less senitive for lasiocarpine and riddelliine, respectively. It is concluded that for quantitative in vitro to in vivo extrapolation (QIVIVE) use of primary hepatocytes is favoured over use of HepaRG cells. For this reason, and also because of the availability of rat in vivo data, in the present study the results obtained in the primary rat hepatocytes were used for the reverse dosimetry.

For this reverse dosimetry we used the AUC instead of the the maximum concentration $\left(\mathrm{C}_{\max }\right)$ as the selected metric. When predicting toxicity for which a threshold exists and thus a concentration below which there will be no effect, the $\mathrm{C}_{\max }$ may be the selected parameter for the reverse dosimetry, while the use of the AUC would be relevant for endpoints like genotoxicity or tumour formation that depend more on the total dose than on peak concentrations (Groothuis et al., 2015). In the present study, the predicted PoDs based on the AUC approach were comparable with available in vivo data on genotoxicity of lasiocarpine and riddelliine in rat liver (Figure 3). When making the prediction based on the $\mathrm{C}_{\max }$ approach, the predicted $\mathrm{BMD}_{10}$ of lasiocarpine and riddelliine were up to 17.4- and 42.2fold lower compared to in vivo animal data. This corroborates that prediction of genotoxicity for the target compound based on the AUC approach is an adequate choice (Groothuis et al., 2015).

It is also of interest to compare the values obtained for in vivo genotoxicity of lasicarpine and riddelliine to PoDs derived from available tumour data. From data on the incidence of liver haemangiosarcoma obtained in 2-year carcinogenicity studies in rats upon chronic oral exposure to 
lasiocarpine and riddelliine (NTP, 1978, 2003). EFSA derived a $\mathrm{BMD}_{10}$ of $0.131 \mathrm{mg} / \mathrm{kg}$ bw/day for lasiocarpine and of $0.292 \mathrm{mg} / \mathrm{kg}$ bw/day for riddelliine (EFSA, 2017). These $\mathrm{BMD}_{10}$ values obtained from the 2-year carcinogenicity studies are one to two orders of magnitude lower than the $\mathrm{BMD}_{10}$ levels predicted in the present study and the dose levels used in other studies to detect genotoxicity (Chan et al., 1994; Xia et al., 2013; Yan et al., 2002) (Figure 3). This may be related to the fact that the exposure duration in the genotoxicity studies is far shorter (1-5 days) than the 2-year exposure applied in carcinogenicity studes.

It is also of interest to consider that in risk assessment for PAs, the estimate daily intake (EDI) values were calculated based on total PA levels in food, taking into consideration combined exposure to different PAs (BfR, 2013; EFSA, 2016, 2017). When considering combined exposure, different potency of the various PAs could be considered. This implies that combined exposure could take their relative potency (REP) into account to 'adjust' the individual PA concentrations. The concept of REP factors describes the relative toxic potency of each congener in comparison to a selected reference congener. The most well established example in toxicology for this approach is the equivalency concept for polychlorinated dioxins, furans and PCBs (Van den Berg et al., 2006). Similar approaches were suggested for phototoxic furocoumarins (Raquet and Schrenk, 2014), or are applied in pharmacology to synthetic glucocorticoids (Suzuki et al., 2015). Recently, Allemang et al. (2018) determined the concentration-dependent PA-induced formation of micronuclei in HepaRG cells by exposing the cells to 15 PAs and calculated the REP values based on these concentration-response curves, lasiocarpine was found to be 6 times more potent than riddelliiine (Allemang et al., 2018). In the present study, we used the $\gamma \mathrm{H} 2 \mathrm{AX}$ assay to detect the genotoxicity of lasiocarpine and riddelliine in primary rat hepatocytes and found that lasiocarpine was 3.5-fold more potent than riddelliine. These REP values, however, are based on in vitro data and do not take the differences in toxicokinetics into account. The results of the present study, in which the concentration-response curves were translated to in vivo dose-response curves using PBK modelling, reveal that the differences in toxicokinetics between different PAs may be substantial and may influence their ultimate in vivo REP values. On the basis of the predicted in vivo rat genotoxicity, riddelliine was found to be more potent (2.6-fold) than lasiocarpine, which is in agreement with in vivo genotoxicity findings.

In conclusion, we demonstrated that the PBK modelling-facilitated reverse dosimetry can adequately translate in vitro concentration-response curves for lasiocarpine and riddelliine, obtained using the $\gamma \mathrm{H} 2 \mathrm{AX}$ assay in primary hepatocytes, to in vivo dose-response curves for genotoxicity. The present study provides an alternative approach for the assessment of other types of PAs for which in vivo genotoxic data are not available, and definition of in vivo REP values, thereby contributing to the reduction, refinement and replacement of animal testing. 


\section{Acknowledgements}

This work was funded by a grant from the China Scholarship Council to Lu Chen (grant No. 201508650023). 


\section{Reference}

Allemang, A., Mahony, C., Lester, C., Pfuhler, S., 2018. Relative potency of fifteen pyrrolizidine alkaloids to induce DNA damage as measured by micronucleus induction in HepaRG human liver cells. Food Chem Toxicol 121, 72-81

Aninat, C., Piton, A., Glaise, D., Le Charpentier, T., Langouët, S., Morel, F., Guguen-Guillouzo, C. and Guillouzo, A., 2006. Expression of cytochromes P450, conjugating enzymes and nuclear receptors in human hepatoma HepaRG cells. Drug Metab Dispos, 34(1), pp.75-83

Audebert, M., Riu, A., Jacques, C., Hillenweck, A., Jamin, E., Zalko, D., Cravedi, J.-P., 2010. Use of the $\gamma \mathrm{H} 2 \mathrm{AX}$ assay for assessing the genotoxicity of polycyclic aromatic hydrocarbons in human cell lines. Toxicol Lett 199, 182-192

Audebert M, Zeman F, Beaudoin R, Pery A, Cravedi JP., 2012. Comparative potency approach based on H2AX assay for estimating the genotoxicity of polycyclic aromatic hydrocarbons. Toxicol Appl Pharmacol 260:58-64

BfR, 2013. Pyrrolizidine alkaloids in herbal teas and teas. BfR Opinion No. 018/2013 of 5 July 2013

Bodi, D., Ronczka, S., Gottschalk, C., Behr, N., Skibba, A., Wagner, M., Lahrssen-Wiederholt, M., Preiss-Weigert, A., These, A., 2014. Determination of pyrrolizidine alkaloids in tea, herbal drugs and honey. Food Addit Contam Part A 31, 1886-1895

Bonner WM, Redon CE, Dickey JS, Nakamura AJ, Sedelnikova OA, Solier S, Pommier Y., 2008. GammaH2AX and cancer. Nat Rev Cancer 8:957-967

Candrian, U., Lüthy, J., Graf, U., Schlatter, C., 1984. Mutagenic activity of the pyrrolizidine alkaloids seneciphylline and senkirkine in Drosophila and their transfer into rat milk. Food Chem Toxicol 22, 223-225

Chan, P.C., Mahler, J., Bucher, J.R., Travlos, G.S. and Reid, J.B., 1994. Toxicity and carcinogenicity of riddelliine following 13 weeks of treatment to rats and mice. Toxicon, 32(8), pp.891-908

Chen, L., Ning, J., Louisse, J., Wesseling, S., Rietjens, IMCM., 2018. Use of physiologically based kinetic modelling-facilitated reverse dosimetry to convert in vitro cytotoxicity data to predicted in vivo liver toxicity of lasiocarpine and riddelliine in rat. Food Chem Toxicol 116, 216-226.

Chen, T., Mei, N., Fu, P.P., 2010. Genotoxicity of pyrrolizidine alkaloids. Journal of Applied Toxicology: An International Journal 30, 183-196.

Chou, M.W., Wang, Y.-P., Yan, J., Yang, Y.-C., Beger, R.D., Williams, L.D., Doerge, D.R., Fu, P.P., 2003. Riddelliine $\mathrm{N}$-oxide is a phytochemical and mammalian metabolite with genotoxic activity that is comparable to the parent pyrrolizidine alkaloid riddelliine. Toxicology letters $145,239-247$.

Chou, M.W., Yan, J., Nichols, J., Xia, Q., Beland, F.A., Chan, P.-C., Fu, P.P., 2004. Correlation of DNA adduct formation and riddelliine-induced liver tumorigenesis in F344 rats and B6C3F1 mice [Cancer Lett. 193 (2003) 119-125]. Cancer letters 207, 119-125.

Clarke, J.J., Lawlor, T.E., Madraymootoo, W., Pant, K., Young, R.R., Wagner III, V.O., Aardema, M.J., 2012. Summary of in vitro genetic toxicology assay results: expected and unexpected effects of recent study design modifications. Environmental and molecular mutagenesis 53, 631-635.

Creeper, J., Mitchell, A., Jubb, T., Colegate, S., 1999. Pyrrolizidine alkaloid poisoning of horses grazing a native heliotrope (Heliotropium ovalifolium). Australian veterinary journal 77, 401402.

Edgar, J.A., Molyneux, R.J., Colegate, S.M., 2014. Pyrrolizidine alkaloids: potential role in the etiology of cancers, pulmonary hypertension, congenital anomalies, and liver disease. Chemical research in toxicology $28,4-20$.

EFSA, 2011. Scientific opinion on pyrrolizidine alkaloids in food and feed. EFSA Journal 9, 2406.

EFSA, 2016. Dietary exposure assessment to pyrrolizidine alkaloids in the European population. EFSA Journal 14, e04572.

EFSA, 2017. Risks for human health related to the presence of pyrrolizidine alkaloids in honey, tea, herbal infusions and food supplements. EFSA Journal 15. 
Fu, P., Chou, M., Xia, Q., Yang, Y.-C., Yan, J., Doerge, D., Chan, P., 2001. Genotoxic pyrrolizidine alkaloids and pyrrolizidine alkaloid N-oxides - mechanisms leading to DNA adduct formation and tumorigenicity. Journal of Environmental Science and Health, Part C 19, 353-385.

Fu, P.P., Chou, M.W., Churchwell, M., Wang, Y., Zhao, Y., Xia, Q., Gamboa da Costa, G.a., Marques, M.M., Beland, F.A., Doerge, D.R., 2010. High-performance liquid chromatography electrospray ionization tandem mass spectrometry for the detection and quantitation of pyrrolizidine alkaloid-derived DNA adducts in vitro and in vivo. Chemical research in toxicology 23, 637-652.

Fu, P.P., Xia, Q., Chou, M.W., Lin, G., 2007. Detection, hepatotoxicity, and tumorigenicity of pyrrolizidine alkaloids in Chinese herbal plants and herbal dietary supplements. Journal of Food and Drug Analysis 15.

Fu, P.P., Xia, Q., Lin, G., Chou, M.W., 2004. Pyrrolizidine alkaloids-genotoxicity, metabolism enzymes, metabolic activation, and mechanisms. Drug metabolism reviews 36, 1-55.

Graillot, V., Takakura, N., Hegarat, L.L., Fessard, V., Audebert, M. and Cravedi, J.P., 2012. Genotoxicity of pesticide mixtures present in the diet of the French population. Environmental and molecular mutagenesis, 53(3), pp.173-184.

Groothuis, F.A., Heringa, M.B., Nicol, B., Hermens, J.L., Blaauboer, B.J. and Kramer, N.I., 2015. Dose metric considerations in in vitro assays to improve quantitative in vitro-in vivo dose extrapolations. Toxicology, 332, pp.30-40.

Guillouzo, A., Corlu, A., Aninat, C., Glaise, D., Morel, F., Guguen-Guillouzo, C., 2007. The human hepatoma HepaRG cells: a highly differentiated model for studies of liver metabolism and toxicity of xenobiotics. Chemico-biological interactions 168, 66-73.

Hill, B., Gaul, K., Noble, J., 1997. Poisoning of feedlot cattle by seeds of Heliotropium europaeum. Australian veterinary journal 75, 360-361.

Hirono, I., Haga, M., Fujii, M., Matsuura, S., Matsubara, N., Nakayama, M., Furuya, T., Hikichi, M., Takanashi, H., Uchida, E., 1979. Induction of hepatic tumors in rats by senkirkine and symphytine. Journal of the National Cancer Institute 63, 469-472.

Hirono, I., Mori, H., Culvenor, C.C., 1976. Carcinogenic activity of coltsfoot, Tussilago farfara L. GANN Japanese Journal of Cancer Research 67, 125-129.

Hoeijmakers, J.H., 2009. DNA damage, aging, and cancer. New England Journal of Medicine 361, $1475-1485$.

Imreh G, Norberg HV, Imreh S, Zhivotovsky B. 2011. Chromosomal breaks during mitotic catastrophe trigger gammaH2AX-ATMp53-mediated apoptosis. J Cell Sci 124:2951-2963.

Kempf, M., Reinhard, A., Beuerle, T., 2010. Pyrrolizidine alkaloids (PAs) in honey and pollen-legal regulation of PA levels in food and animal feed required. Molecular nutrition \& food research $54,158-168$.

Khoury, L., Zalko, D., Audebert, M., 2013. Validation of high-throughput genotoxicity assay screening using $\gamma \mathrm{H} 2 \mathrm{AX}$ in-cell western assay on HepG2 cells. Environmental and molecular mutagenesis 54, 737-746.

Kim, H.Y., Stermitz, F.R. and Coulombe Jr, R.A., 1995. Pyrrolizidine alkaloid-induced DNA-protein cross-links. Carcinogenesis, 16(11), pp.2691-2697.

Kopp, B., Zalko, D. and Audebert, M., 2018. Genotoxicity of 11 heavy metals detected as food contaminants in two human cell lines. Environmental and molecular mutagenesis, 59(3), pp.202-210.

Kuhara, K., Takanashi, H., Hirono, I., Furuya, T., Asada, Y., 1980. Carcinogenic activity of clivorine, a pyrrolizidine alkaloid isolated from Ligularia dentata. Cancer letters 10, 117-122.

Lin, G., Cui, Y.-Y., Liu, X.-Q., 2003. Gender differences in microsomal metabolic activation of hepatotoxic clivorine in rat. Chemical research in toxicology 16, 768-774.

Lin, G., Wang, J.Y., Li, N., Li, M., Gao, H., Ji, Y., Zhang, F., Wang, H., Zhou, Y., Ye, Y., 2011. Hepatic sinusoidal obstruction syndrome associated with consumption of Gynura segetum. Journal of hepatology 54, 666-673. 
Louisse J, Rijkers D, Stoopen G, Jansen HWendy, Delagrange M, Peijnenburg A (Submitted) Determination of genotoxic potencies of pyrrolizidine alkaloids in HepaRG cells using the $\gamma \mathrm{H} 2 \mathrm{AX}$ assay. Food Chem Toxicol

Mathon, C., Edder, P., Bieri, S., Christen, P., 2014. Survey of pyrrolizidine alkaloids in teas and herbal teas on the Swiss market using HPLC-MS/MS. Analytical and bioanalytical chemistry 406, 7345-7354.

Mattocks, A., 1986. Chemistry and toxicology of pyrrolizidine alkaloids. Academic Press.

Molyneux, R., Gardner, D., Colegate, S., Edgar, J., 2011. Pyrrolizidine alkaloid toxicity in livestock: a paradigm for human poisoning? Food Additives \& Contaminants: Part A 28, 293-307.

Mori, H., Sugie, S., Yoshimi, N., Asada, Y., Furuya, T., Williams, G.M., 1985. Genotoxicity of a variety of pyrrolizidine alkaloids in the hepatocyte primary culture-DNA repair test using rat, mouse, and hamster hepatocytes. Cancer Research 45, 3125-3129.

NTP, 1978. Bioassay of lasiocarpine for possible carcinogenicity. National Cancer Institute carcinogenesis technical report series 39,1 .

NTP, 2003. Toxicology and carcinogenesis studies of riddelliine (CAS No. 23246-96-0) in F344/N rats and B6C3F1 mice (gavage studies). National Toxicology Program technical report series, 1.

Ning, J., Chen, L., Strikwold, M., Louisse, J., Wesseling, S. and Rietjens, I.M., 2019. Use of an in vitro-in silico testing strategy to predict inter-species and inter-ethnic human differences in liver toxicity of the pyrrolizidine alkaloids lasiocarpine and riddelliine. Archives of toxicology, pp.1-18.

O'donovan, M., 2012. A critique of methods to measure cytotoxicity in mammalian cell genotoxicity assays. Mutagenesis 27, 615-621.

Prakash, A.S., Pereira, T.N., Reilly, P.E., Seawright, A.A., 1999. Pyrrolizidine alkaloids in human diet. Mutation Research/Genetic Toxicology and Environmental Mutagenesis 443, 53-67.

Roeder, 2000. Medicinal plants in China containing pyrrolizidine alkaloids. Pharmazie 55, I0.

Shumaker, R., Robertson, K., Hsu, I., Allen, J., 1976. Neoplastic transformation in tissues of rats exposed to monocrotaline or dehydroretronecine. Journal of the National Cancer Institute 56, 787-790.

Sedelnikova, O.A., Redon, C.E., Dickey, J.S., Nakamura, A.J., Georgakilas, A.G. and Bonner, W.M., 2010. Role of oxidatively induced DNA lesions in human pathogenesis. Mutation Research/Reviews in Mutation Research, 704(1-3), pp.152-159.

Singh SK, Wang M, Staudt C, Iliakis G. 2011. Post-irradiation chemical processing of DNA damage generates double-strand breaks in cells already engaged in repair. Nucleic Acids Res 39: 84168429 .

Takanashi et al., (1980) Chromosomal aberrations and mutation in cultured mammalian cells induced by pyrrolizidine alkaloids. Mutat. Res. 78,67-77.

Theumer, M.G., Henneb, Y., Khoury, L., Snini, S.P., Tadrist, S., Canlet, C., Puel, O., Oswald, I.P. and Audebert, M., 2018. Genotoxicity of aflatoxins and their precursors in human cells. Toxicology letters, 287, pp.100-107.

Turpeinen, M., Tolonen, A., Chesne, C., Guillouzo, A., Uusitalo, J., Pelkonen, O., 2009. Functional expression, inhibition and induction of CYP enzymes in HepaRG cells. Toxicology in Vitro 23, 748-753.

Uhl, M., Helma, C., Knasmüller, S., 2000. Evaluation of the single cell gel electrophoresis assay with human hepatoma (Hep G2) cells. Mutation Research/Genetic Toxicology and Environmental Mutagenesis 468, 213-225.

Van den Berg, M., Birnbaum, L.S., Denison, M., De Vito, M., Farland, W., Feeley, M., Fiedler, H., Hakansson, H., Hanberg, A., Haws, L. and Rose, M., 2006. The 2005 World Health Organization reevaluation of human and mammalian toxic equivalency factors for dioxins and dioxin-like compounds. Toxicological sciences, 93(2), pp.223-241.

Wang, Y.-P., Fu, P.P., Chou, M.W., 2005a. Metabolic activation of the tumorigenic pyrrolizidine alkaloid, retrorsine, leading to DNA adduct formation in vivo. International journal of environmental research and public health 2, 74-79. 
Wang, Y.-P., Yan, J., Beger, R.D., Fu, P.P., Chou, M.W., 2005b. Metabolic activation of the tumorigenic pyrrolizidine alkaloid, monocrotaline, leading to DNA adduct formation in vivo. Cancer letters 226, 27-35.

Wiedenfeld, H., 2011. Plants containing pyrrolizidine alkaloids: toxicity and problems. Food Additives \& Contaminants: Part A 28, 282-292.

Xia, Q., Chou, M.W., Edgar, J.A., Doerge, D.R., Fu, P.P., 2006. Formation of DHP-derived DNA adducts from metabolic activation of the prototype heliotridine-type pyrrolizidine alkaloid, lasiocarpine. Cancer letters 231, 138-145.

Xia, Q., Zhao, Y., Von Tungeln, L.S., Doerge, D.R., Lin, G., Cai, L., Fu, P.P., 2013. Pyrrolizidine alkaloid-derived DNA adducts as a common biological biomarker of pyrrolizidine alkaloidinduced tumorigenicity. Chemical research in toxicology 26, 1384-1396.

Yan, J., Nichols, J., Yang, Y.-C., Fu, P.P., Chou, M.W., 2002. Detection of riddelliine-derived DNA adducts in blood of rats fed riddelliine. International Journal of Molecular Sciences 3, 10191026. 


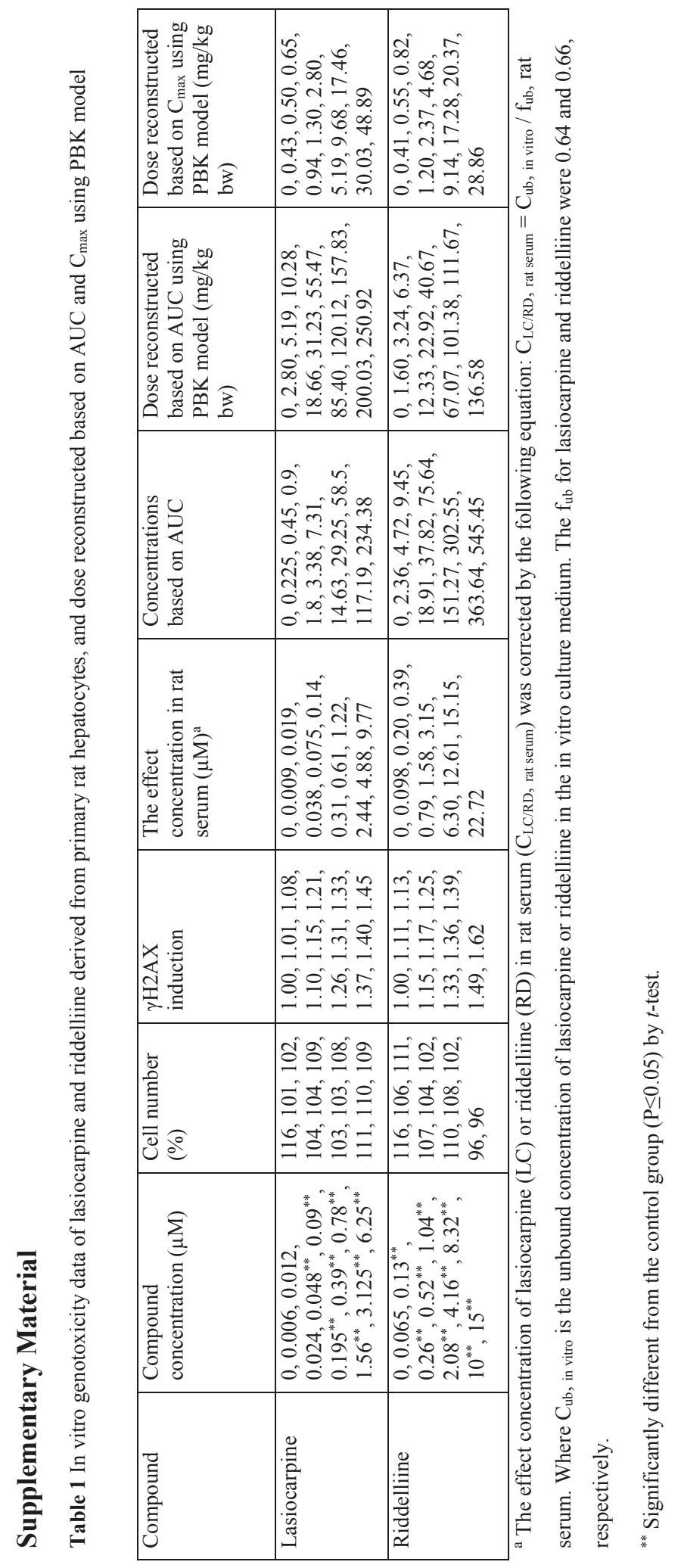





\section{Chapter 7}

General discussion 


\section{Major findings}

One of the aims of this thesis was to perform a risk assessment for PAs derived from botanical products following daily life-time exposure and more realistic exposure scenarios. Another aim was to develop a novel animal-free approach to predict in vivo liver toxicity and genotoxicity of PAs using lasiocarpine and riddelliine as two model PAs. The botanical products used in this thesis were herbal teas, herbal medicines and plant food supplements (PFS), because consumption of these botanical products is thought to represent major routes for human exposure to PAs (Bodi et al., 2014; Roeder, 2000). Given that 1,2-unsaturated PAs are genotoxic and carcinogenic, their risk assessment is currently based on the Margin of Exposure (MOE) approach (EFSA, 2005). In this approach daily lifetime exposure is the default assumption, but such an assumption may not be fully adequate as people might rather consume herbal products occasionally or only during certain periods, for example illness. Application of the MOE approach combined with Haber's rule was used to take shorter than life-time, i.e. more realistic, exposure scenarios into account. Another consideration was that consumption of herbal teas usually occurs by hot water extraction of partially intact or coarsely ground leaves, so that extraction of comminuted leaves for exposure and subsequent risk assessment may represent a worst case situation. Another bottle neck encountered in the risk assessment of PAs is that exposure is often to a mixture of different PAs of different potency while data to define their in vivo relative potency (REP) factors are lacking. Lack of data is a general problem in the field of PA toxicity since only for a limited number of PAs data on liver toxicity, genotoxicity and/or carcinogenicity are available. This is why the present thesis also investigated whether alternative testing strategies could be of use in predicting in vivo toxicity of different PAs and whether proofs-of-principle for using such alternative testing strategies could be provided for two selected PA model compounds, riddelliine and lasiocarpine. These PAs were selected because for these PAs in vivo data enabling evaluation of the in vivo predictions are available at least to some extent. The alternative method used to predict in vivo liver toxicity and genotoxicity of lasiocarpine and riddelliine, is based on extrapolation of concentration-response curves obtained from in vitro cytotoxicity and genotoxicity assays to in vivo dose-response curves using physiologically based kinetic (PBK) modelling-based reverse dosimetry. From the predicted in vivo dose-response curves points of departure $(\mathrm{PoD})$ for risk assessment can be derived and compared to PoDs derived from data obtained in in vivo studies reported in the literature.

In Chapter 2, a risk assessment based on the MOE approach was performed using the $\mathrm{BMDL}_{10}$ (benchmark dose lower confidence limit for $10 \%$ extra risk on tumour formation above background levels) of $0.07 \mathrm{mg} / \mathrm{kg}$ bw/day for lasiocarpine derived by EFSA (EFSA, 2011) from data established in a 2-year carcinogenicity study on induction of liver haemangiosarcomas in male rats, and mean PA levels reported in literature for herbal teas and PFS. The results indicated that consumption of one cup of tea a day during a whole lifetime would result in MOE values lower than 10000 for several types of herbal teas, indicating a priority for risk management for these products. A refined risk assessment 
using interim REP factors for different PAs was also performed and showed that based on the mean PA levels, 7 (54\%) of 13 types of herbal teas and 1 (14\%) of 7 types of PFS resulted in MOE values lower than 10000 . The herbal preparations that raised a concern included preparations containing PAproducing plants but also preparations containing non-PA-producing plants. In addition, a literature review on tumour data for PAs was provided summarizing tumour data available on lasiocarpine, riddelliine, monocrotaline, clivorine, senkirkine and symphytine. Except for the data on lasiocarpine and riddelliine, these data were not suitable for BMD analysis but enabled definition of T10 values (dose levels causing 10\% extra tumour incidence above background levels upon life time exposure). The T10 values of monocrotaline, clivorine, senkirkine and symphytine were 21-, 4-, 34- and 60- fold higher, respectively, than the $\mathrm{BMDL}_{10}$ value of $0.07 \mathrm{mg} / \mathrm{kg}$ bw/day for lasiocarpine. These values support that the $\mathrm{BMDL}_{10}$ for lasiocarpine of $0.07 \mathrm{mg} / \mathrm{kg}$ bw/day is the lowest PoD and thus seems a (too) conservative estimate when applied for other PAs without correcting for differences in relative potencies. The data resulted in REP values for lasiocarpine, riddelliine, monocrotaline, clivorine, senkirkine and symphytine that amounted to $1.00,0.39,0.05,0.23,0.03,0.02$. In a later opinion EFSA referred to our study as well as to the paper on interim REP values reported by Merz and Schrenk (Merz and Schrenk, 2016) stating that although the REP values presented were not robust enough to be taken into account in risk assessment of combined exposure, they did reveal that using the $\mathrm{BMDL}_{10}$ for lasiocarpine of $0.07 \mathrm{mg} / \mathrm{kg}$ bw/day may be too worst case (EFSA, 2017). Moreover, EFSA suggested the use of the newly derived $\mathrm{BMDL}_{10}$ of $0.24 \mathrm{mg} / \mathrm{kg}$ bw/day for riddelliine instead. It is of interest to note that the interim REP values proposed by Merz and Schrenk were based on in vitro cytotoxicity, genotoxicity in Drosophila, and acute toxicity in rodents (LD50) so they did not reflect the expected in vivo differences in carcinogenicity between the different PAs, the critical effect evaluated when performing a risk assessment for chronic toxicity using the MOE. This lack of data to characterise the in vivo relative potencies of PAs provides another reason to work towards methods that can predict relative differences in in vivo potency as aimed for in the present thesis.

Given that the use of botanical products during every day of a whole life-time may not be realistic, we also investigated methods to correct for shorter-than-lifetime exposure (Chapter 3). Use of the MOE approach with the $\mathrm{BMDL}_{10}$ of $0.24 \mathrm{mg} / \mathrm{kg}$ bw/day for riddelliine and total PA levels, combined with Haber's rule was employed to analyse the risks of shorter-than-lifetime exposure for 5 herbal teas, 8 herbal medicines, 34 previously analysed herbal teas and 19 PFS (Bodi et al., 2014; EFSA, 2016; Mulder et al., 2015). This analysis revealed that shorter-than-lifetime use would result in MOE values lower than 10000 upon use for 40 up to 3450 weeks during a lifetime (depending on the preparation). Only for a limited number of herbal teas and medicines, use of two weeks a year (150 weeks during a 75 year lifetime) would still raise a concern. When considering the fact that consumption of teas usually occurs by hot water extraction of partially intact or coarsely ground leaves, it is conceivable that use of PAs from comminuted leaves may facilitate their extraction from the teas. This may influence the actual exposure and corresponding risk assessment. Five types of herbal teas were used 
to compare the total amount of PAs that were extracted either from the intact or the comminuted leaves. The results showed that the PA levels extracted from intact leaves were 1.1- to 4.1-fold lower than from the corresponding comminuted leaves, so that it was concluded that the size of the leaves may have an impact on the relative extraction efficiency of PAs present in the leaves, and thus on the resulting exposure and risk assessment.

To develop and validate methods for quantitative in vitro to in vivo extrapolation (QIVIVE), further studies of the present thesis focussed on prediction of in vivo liver toxicity for lasiocarpine and riddelliine in rat (Chapter 4). To this end, the in vitro cytotoxicity of lasiocarpine and riddelliine was determined in primary rat hepatocytes. Then, PBK models were developed that described the clearance of lasiocarpine and riddelline in rat. The kinetic parameters were collected from in vitro incubations using rat liver microsomes. The developed PBK models were used for translation of in vitro concentration-response curves to in vivo dose-response curves. From these in vivo dose-response curves, the predicted $\mathrm{BMDL}_{5}-\mathrm{BMDU}_{5}$ (lower/upper limit of the $90 \%$ confidence interval of the benchmark dose that gives a $5 \%$ response) of lasiocarpine and riddelline were obtained which were 23.0-34.4 and 4.9-8.4 mg/kg bw/day, respectively. Due to the absence of in vivo acute liver toxicity data reported in the literature for riddelliine, the predicted PoDs for in vivo liver toxicity could only be evaluated for lasiocarpine. To this end, the predicted $\mathrm{BMDL}_{5}-\mathrm{BMDU}_{5}$ of lasiocarpine were compared with in vivo reported data for acute liver toxicity of lasiocarpine as available in the literature (Dalefield et al., 2016; Jago, 1970; Nolan et al., 1966). The predicted BMDL 5 of lasiocarpine was 1.9to 2.9-fold higher than the NOAELs (no observed adverse effect level) derived as corresponding PoD from the available experimental in vivo data. Overall, the predicted $\mathrm{BMDL}_{5}-\mathrm{BMDU}_{5}$ of lasiocarpine falls well within the range of the PoDs derived from available in vivo toxicity data. The results indicate that the data and range of PoD values derived using our combined in vitro-PBK modelling approach are a good approximation for the in vivo acute liver toxicity of lasiocarpine. In addition, the $\mathrm{BMDL}_{5}-\mathrm{BMDU}_{5}$ values predicted for riddelliine were 2.7 to 7.0 times lower than those values predicted for lasiocarpine. This indicates that in vivo riddelliine is predicted to induce liver toxicity at lower dose levels than lasiocarpine. In line with this, the in vitro cytotoxicity of riddelliine was also higher than that of lasiocarpine. The higher in vivo toxicity of riddelliine may in part be due to the fact that there are differences in kinetics which revealed a slower clearance of riddelliine compared to lasiocarpine. This observation indicates that when defining relative potencies for differences in in vivo toxicity, such differences in toxicokinetics should be taken into account. It is reasonable to expect that the approach, now validated for lasiocarpine, can also provide adequate data for the in vivo toxicity of other related PAs.

The same QIVIVE method was subsequently used to predict the inter-species and inter-ethnic 
human differences in liver toxicity of lasiocarpine and riddelliine using a human PBK model for reverse dosimetry of in vitro toxicity data obtained in human primary hepatocytes (Chapter 5). The concentration-response curves for in vitro cytotoxicity of lasiocarpine and riddelliine defined in pooled primary human hepatocytes were translated to in vivo dose-response curves by PBK models developed using kinetic data obtained from incubations with pooled tissue fractions from Chinese and Caucasian individuals, providing PBK models for the average Chinese and average Caucasian, respectively. From the predicted in vivo dose-response curves, the $\mathrm{BMDL}_{5}-\mathrm{BMDU}_{5}$ of lasiocarpine were found to amount to $14.7-41.2 \mathrm{mg} / \mathrm{kg}$ bw/day for Chinese and 7.4-23.7 mg/kg bw/day for Caucasian, indicating the Chinese to be less sensitive. The predicted $\mathrm{BMDL}_{5}-\mathrm{BMDU}_{5}$ of riddelliine were 1.0-5.9 mg/kg bw/day for Chinese and $0.2-1.2 \mathrm{mg} / \mathrm{kg}$ bw/day for Caucasian. These values were subsequently compared to those previously obtained in rat to evaluate inter-species differences. The inter-species differences amounted to 2.0-fold for lasiocarpine and 8.2-fold for riddelliine with humans being more sensitive than rats. The inter-ethnic human differences varied 2.0-fold for lasiocarpine and 5.0-fold for riddelliine with the average Caucasian being more sensitive than the average Chinese. These results provide a proof-of-principle for predicting inter-species and inter-ethnic differences in in vivo liver toxicity for PAs by an alternative testing strategy integrating in vitro cytotoxicity data with PBK modelling-based reverse dosimetry.

In a subsequent study of the present thesis the same approach was used for prediction of genotoxicity, another endpoint relevant for PA toxicity (Chapter 6). State-of-the-art genotoxicity data on PAs are limited, and lack of genotoxicity studies for most 1,2-unsaturated PAs, hampers PA risk assessment, while economic and ethical constraints limit the possibilities to perform in vivo genotoxicity studies for all relevant PAs, including the 17 PAs identified by EFSA to be relevant for exposure via feed and food (EFSA, 2017). Thus, alternative testing strategies for estimating the genotoxicity of different PAs are required. It is obvious to investigate whether the developed PBK modelling-facilitated reverse dosimetry approach developed in Chapter 4 can quantitatively translate in vitro concentration-response curves for genotoxicity of lasiocarpine and riddelliine, to in vivo dose-response curves for genotoxicity of these PAs in rat liver. To this end, the in vitro concentration-responses curves obtained from in vitro genotoxicity studies with primary rat hepatocytes using the $\gamma \mathrm{H} 2 \mathrm{AX}$ assay were translated into in vivo dose-response curves using PBK modelling-facilitated reverse dosimetry. The predicted $\mathrm{BMD}_{10}$ for lasiocarpine and riddelliine amounted to 8.82 and $3.41 \mathrm{mg} / \mathrm{kg}$ bw/day, respectively, and were in line with the experimental data on in vivo genotoxicity available in the literature for these two PAs (Chan et al.,1994; Xia et al., 2013; Yan et al., 2002). Lasiocarpine was found to be 6 times more potent than riddelliine based on results from an in vitro micronuclei assay (Allemang et al., 2018). However, when taking the differences in toxickinetics into account, the predicted in vivo genotoxicity of riddelliine was found to be 2.6-fold higher than that of lasiocarpine. These results reveal that the differences in toxicokinetics between different PAs may be substantial and may influence their ultimate in vivo REP values. It is concluded that integrating in vitro genotoxicity assays with PBK 
modelling-facilitated reverse dosimetry provides a method to predict in vivo genotoxicity for the large number of PAs for which in vivo genotoxicity data are lacking.

\section{Major discussion points}

The results obtained in the present study show some important steps forward in the risk assessment of PAs but at the same time also raise issues for further consideration and future research. The next sections provide an overview of these future challenges and their perspectives.

\section{Cut off value in the MOE approach based risk assessment}

To assess the risk to human health of compounds that are both genotoxic and carcinogenic based on data derived from animal experiments, the MOE approach was developed (EFSA, 2005). The MOE is defined as the ratio between the $\mathrm{BMDL}_{10}$ and the estimated daily intake (EDI). An MOE value higher than 10000 is considered as a low priority for risk management actions and would be of low concern from a public health point of view (EFSA, 2005). The cut-off value of 10000 was defined taking several factors into account, including a factor 100 for species differences and human variability in toxicokinetics and toxicodynamics, a factor 10 for inter-individual human variability in cell cycle control and DNA repair, and a factor 10 because the $\mathrm{BMDL}_{10}$ when used as a reference point is not identical to a no effect level (EFSA, 2005; O'Brien et al., 2006). The proposed default value of 10 for interspecies differences consists of a factor of 4 for differences between species in toxicokinetics and 2.5 for interspecies differences in toxicodynamic (IPCS, 2005). However, whether this factor of 10 is suitable for all the genotoxic carcinogens may need further investigations defining compound specific assessment factors (CSAFs). In Chapter 4 and 5, an in vitro-PBK model based approach was developed to predict in vivo liver toxicity for lasiocarpine and riddelliine in rat and human, showing that lasiocarpine and riddelliine induced liver toxicity was predicted to be 2-fold and 8-fold higher in human than in rat, indicating humans to be somewhat more sensitive towards acute liver toxicity of lasiocarpine and riddelliine than rats. The higher toxicity of humans may be due to the kinetics that resulted in a 1.9- and 5.0-fold slower clearance of lasiocarpine and riddelliine in humans compared to rats. The major species-specific differences in susceptibility to PA toxicity may be attributed to several toxicokinetic factors such as CYP-mediated capacity of bioactivation, efficiency of N-oxidation, and carboxylesterase-mediated hydrolysis as well as the level of cellular GSH (Fu et al., 2004; Huan et al.,1998; Stegelmeier et al., 1999; Duringer et al., 2004; Winter et al., 1998). Fashe et al. (2015) examined the species-dependent variations in the metabolic detoxification and bioactivation of lasiocarpine. The results showed that the relative amount of the main demethylation metabolite (M9) as the major detoxification product of lasiocarpine was lower with human liver microsomes than with the liver microsomes of other species including rats, and more GSH reactive metabolites were formed with human liver microsomes (Fashe et al., 2015). The results obtained reveal that when extrapolating rodent toxicity data for lasiocarpine and riddelliine to the human situation, the proposed default value of 10 for interspecies differences is adequately or perhaps even somewhat overprotective because 
humans were shown to be 2- and 8-fold more sensitive than rats. A CSAF that could be smaller than the default value of 10 could thus be defined, especially for lasiocarpine. Then the default MOE value of 10000 may decrease accordingly which would result in less botanical product samples to result in MOE values that might raise a concern upon lifetime use. One could argue that when the uncertainty factor for interspecies differences of 10 could be reduced to 2 or 8 an MOE of 2000 or 8000 could be used as a cut off value. As outlined by EFSA when introducing the MOE approach selection of the cut off value is ultimately a matter for the risk managers (EFSA, 2005). The results of the present thesis indicate that use of QIVIVE based on PBK modelling may prove a way forward in defining CSAFs and thus also, compound specific MOE cut off values in risk assessment.

\section{Combined exposure to PAs}

Exposure to PAs generally includes exposure to a mixture of different congeners. EFSA identified 17 PAs to be especially relevant for exposure via food and feed including also lasiocarpine as one of the two model compounds of the present thesis (EFSA, 2017). Close analysis of the type of PAs present in the different herbal products revealed that in the majority of samples lasiocarpine and riddelliine did not represent a major PA or were even absent (Bodi et al., 2014; EFSA, 2016; Mulder et al., 2015, 2018) (Chapter 3). However, for the majority of the 17 PAs identified by EFSA to be important in food and feed, tumour data and related PoDs are absent hampering their risk assessment. So far, amongst all PAs only for lasiocarpine and riddelliine 2-year animal bioassays have been performed and provided data that are suitable to derive $\mathrm{BMDL}_{10}$ values for induction of liver tumours (NTP, 1978, 2003). Whether the $\mathrm{BMDL}_{10}$ for lasiocarpine or riddelliine is the most appropriate or rather a too conservative PoD for risk assessment remains to be established. As already outlined above, EFSA reconsidered the use of the $\mathrm{BMDL}_{10}$ of $0.07 \mathrm{mg} / \mathrm{kg}$ bw/day for lasiocarpine and proposed to use of the $\mathrm{BMDL}_{10}$ of $0.24 \mathrm{mg} / \mathrm{kg}$ bw/day for riddelliine for the combined risk assessment of PAs by dose addition. Determining REP factors for the different PAs and using these to adjust exposure values in a dose-addition concept would be a way forward in the risk assessment of PA-containing botanicals. The interim REP factors for PAs defined by Merz and Schrenk, (2016) were based on data from in vitro cytotoxicity, genotoxicity in Drosophila, and acute toxicity in rodents (LD50). From these data, REPs were derived for structurally related subgroups of PAs, amounting to 1.0 for cyclic diesters and heliotridine-type (7S) open diesters (based on data for monocrotaline, retrorsine, riddelliine, senecionine, seneciphylliine, senkirkine, heliosupine and lasiocarpine). 0.3 for heliotridine-type (7S) monoesters (based on data for echinatine and heliotrine), 0.1 for retronecine-type (7R) open diesters (based on data for echimidine and symphytine), and 0.01 for retronecine-type (7R) monoesters (based on data for indicine, intermedine and lycopsamine) (Merz and Schrenk, 2016). Recently, Allemang et al. (2018) determined the concentration-dependent PA-induced formation of micronuclei in HepaRG cells by exposing the cells to 15 PAs and calculated the REP values based on these concentrationresponse curves (Allemang et al., 2018). In both studies lasiocarpine and riddelliine have a relatively 
high potency, but lasiocarpine was found to be 6 times more potent than riddelliine in the micronucleus study. In the present thesis, the $\gamma \mathrm{H} 2 \mathrm{AX}$ assay was used to detect genotoxicity of lasiocarpine and riddelliine in primary rat hepatocytes, and translation of the concentration-response curves to in vivo dose-response curves was based on PBK model facilitated reverse dosimetry. Based on the in vitro genotoxicity data thus obtained lasiocarpine was 3.5 -fold more potent than riddelliine. However when differences in kinetics were taken into account and the in vitro data were translated to the in vivo situation the predicted dose-response curves, riddelliine was found to be 2.6-fold more potent than lasiocarpine (Chapter 6). This difference could be ascribed to a far more efficient clearance of lasiocarpine as compared to riddelliine with the catalytic efficiency for clearance being 11-fold higher for lasiocarpine. This indicates that definition of REP values based on in vitro data without taking possible differences in toxicokinetics into account may result in inaccurate values. Preferably REP values used in the MOE based risk assessment of combined exposure to PAs should be derived from in vivo carcinogenicity potencies. However, this seems unrealistic given the large number of PAs for which actual carcinogenicity data are lacking. More recently, EFSA re-calculated the $\mathrm{BMDL}_{10}$ values for lasiocarpine and riddelliine based on the 2-year carcinogenicity data, indicating that the previously obtained $\mathrm{BMDL}_{10}$ of lasiocarpine was affected by a high degree of uncertainty (EFSA, 2017). Despite the large difference between the $\mathrm{BMDL}_{10}$ for lasiocarpine and riddelliine, a partial overlap of the $\mathrm{BMDL}_{10}-\mathrm{BMDU}_{10}$ confidence intervals calculated using model averaging was observed, suggesting that the carcinogenic potency of these two PAs could be quite similar. This was more evident when the BMD analysis of the available tumour data was performed using a BMR falling within the tested dose ranges for both substance, such as a $\mathrm{BMD}_{30}$ the dose level resulting in $30 \%$ extra tumour incidence above background values. In this analysis $\mathrm{BMD}_{30}$ values of 0.491 and 0.435 $\mathrm{mg} / \mathrm{kg}$ bw/day were calculated for lasiocarpine and riddelliine, respectively (EFSA, 2017). This additional modelling supported the assumption that the two PAs can be considered of similar carcinogenic potency (EFSA, 2017). One possible explanation for this similarity could be that the same 4 types of DHP-derived DNA adducts were produced in rats exposed to lasiocarpine and riddelliine, which might result in similar chances on gene mutations and tumour formation (Xia et al., 2013; Chen et al., 2010). Together these results show that REP values should not be based on in vitro concentration-response curves without taking differences in kinetics into account. For better refinement, the REP factors could be based on data for genotoxicity of PAs, combined with PBK modelling. Such an in vitro-in silico approach could be applied for different PAs, especially the 17 PAs identified by EFSA to be relevant for exposure via feed and food (EFSA, 2017). Using PBK model based reverse dosimetry for QIVIVE, the toxicokinetics of PAs can be taken into account and the estimated REP for PAs could be closer to the values representing differences in their in vivo potencies. 


\section{Banding of the MOE values for risk management}

MOE values obtained for herbal products in the present thesis showed wide variation, for example, the MOE values for herbal teas varied from 100 to 550000 (Chapter 3). At the current state-of-the-art there is no clear definition of interpretation of MOE values that differentiate from 10000 to a different degree. This implies that an MOE far below 10000 of for example 10, or close to 10000 of for example 9990, are both judged to be of concern, while an MOE of 10005 or 100000 are both of no concern, while these values obviously may represent different risk levels for human health. A banding system has been proposed by the Committee on Carcinogenicity of Chemicals in Food, Consumer Products and the Environment (COC) to assist risk management. The proposed MOE banding is as follow: MOE lower than 10000: may be a concern, MOE value in the range of 10000-1000000: unlikely to be a concern, MOE value higher than 1000000: very unlikely to be a concern (COC, 2012). This system helps the discrimination of the different risk levels above 10000, but it does not consider the situation of MOE values below 10000. One could consider further banding of MOE cut-off values that are lower than 10000 as follows: MOE lower than 1000: a very high concern; MOE values in the range of 1000-5000: a high concern; MOE values in the range of 5000-10000: a concern. When applying this MOE banding, in total 3 (7\%) of 39 types of herbal teas, 3 (38\%) of 8 types of herbal medicines and $1(5 \%)$ of 19 types of PFS resulted in MOE values lower than 1000 based on daily consumption during a whole lifetime, indicating a very high concern for human health (Chapter 3). These examples show how this banding may help the discrimination of the risks of MOE values below 10000.

\section{Shorter-than-lifetime exposure}

Another item that appeared relevant to consider is the fact that exposure to herbal products may not always be relevant during every day of a whole life time. This implies that methods to consider shorter-than-lifetime exposure need to be considered and developed. Since MOE values represent a risk assessment of human health considering daily exposure during a whole life span, this assumption can cause overestimation of the risk for exposure to certain toxic compounds when realistic consumption patterns would imply a period shorter-than-lifetime. This holds especially for adverse effects that are dependent on the total exposure, such as genotoxicity and carcinogenicity, rather than on maximum plasma levels. In Chapter 3, Haber's rule was applied to assess the potential risk for human exposure to PAs derived from consumption of herbal products for periods shorter-than-lifetime. The application of Haber's rule is based on the assumption that the tumour incidence and carcinogenic processes induced by genotoxic carcinogens have a linear relationship with the cumulative dose (Crump et al., 1976). When assuming lifelong daily consumption, 10 out of 39 herbal teas, 3 out of 8 herbal medicines and 2 out of 19 PFS had MOE values below 10000, indicating that these herbal products may pose a potential risk for human health. Considering shorter-than-lifetime exposure for 2 weeks/year and after applying Haber's rule for correction of the exposure values, only 4 types of 
herbal teas and 1 type of herbal medicines resulted in MOE values lower than 10000, all the PFS had MOE values above 10000. Thus, the use of the MOE based on life-time exposure estimates might overestimate the actual risk for human health. Based on the considerations discussed above it is concluded that it would be encouraged to further refine the MOE approach, developing generally accepted methods that take shorter than life-time exposure into account.

\section{The in vitro system selected for quantitative in vitro to in vivo extrapolation (QIVIVE)}

In QIVIVE based on in vitro-PBK modelling-based reverse dosimetry, there are several issues that need to be considered. A first one is selection of a cell model that contains sufficient levels of enzymes involved in PA biotransformation, given that the expression of the relevant biotransformation enzymes may differ between the different cell models or may even be completely absent. The biotransformation capacity of a cell model is considered to be an important issue in in vitro testing (Coecke et al., 2013). A second issue to consider is that the critical effect quantified in the in vitro model and the underlying mode of action should be relevant for the in vivo response and can thus provide a basis for estimation of the in vivo PoD. For prediction of in vivo liver toxicity for lasiocarpine and riddelliine in human, we selected cell-based liver models including HepaRG cells and primary human hepatocytes to generate the in vitro data (Chapter 5). HepaRG cells are derived from a human hepatocellular carcinoma composed of a mixture of both hepatocytes-like and biliary-like cells. The HepaRG cell model contains most of the active CYP450 enzymes, especially the CYP 3A and 2B isoforms that are key enzymes for PAs bioactivation (Fu et al., 2004; Guillouzo et al., 2007; Lin et al., 2003; Prakash et al., 1999; Turpeinen et al., 2009). Primary human hepatocytes represent a unique in vitro system and serve as a 'gold standard' for studies of drug metabolism and toxicity, and are often used by default for hepatotoxicity studies (LeCluyse, 2001). It was demonstrated that the HepaRG cells were able to maintain hepatic functions comparable to primary human hepatocytes (Lübberstedt et al. 2010). Comparisons of the activity of CYP450s in HepaRG cells and primary human hepatocytes have been reported, but the results were inconsistent across different studies. Gerets et al. (2012) reported that CYP 3A4 activity was about 17.0-fold lower in the HepaRG cells than in primary human hepatocytes from three different donors (Gerets et al., 2012). However, Kvist et al. (2018), and Lubberstedt et al. (2011) investigating the CYP 1A2, CYP 2B6, CYP 2C8, CYP 2C9, CYP 2C19 and CYP 2D6 activities in HepaRG cells and primary human hepatocytes, showed that these enzyme activities were generally lower in HepaRG cells than in human hepatocytes, except for CYP 3A4 showing a generally 1.5-fold higher activity in HepaRG cells (Kvist et al., 2018, Lübberstedt et al., 2011). Comparison of the lasiocarpine and riddelliine induced cytotoxicity between HepaRG cells and primary human hepatocytes, revealed primary human hepatocytes to be 10- and 3-fold more sensitive to lasiocarpine and riddelliine induced cytotoxicity than the HepaRG cells. On the other hand, quantifying $\gamma \mathrm{H} 2 \mathrm{AX}$ formation as the endpoint for genotoxicity, the primary rat hepatocytes appeared to be about one to 
two orders of magnitude more sensitive to lasiocarpine and riddelliine induced genotoxicity than the HepaRG cells (Chapter 6). Combining the results from these two Chapters, it is concluded that HepaRG cells have lower metabolic capacity for bioactivation of the PAs compared to primary hepatocytes. One could argue that a large inherent variation of primary human hepatocytes due to the inter-individual variability between different donors as well as the limited supply of high quality donor tissues may lead to a compromised experimental result (Shimada et al., 1994; Zuo et al., 2017). For better understanding of human liver function using in vitro models and to overcome the disadvantage of using primary human hepatocytes, new cell culture technologies such as induced pluripotent stem cells (iPSCs) (Wong et al., 2018), 3D cell culture systems (Bell et al., 2016), 3D-bioreactor technology (Knöspel et al., 2016) and organ-on-a-chip devices (Kimura et al., 2018) can be considered, although it remains to be established whether CYP levels in these models will adequately support PA bioactivation.

Another challenge in the present thesis was to quantitatively define PoDs for the in vivo situation. In Chapter 6, the $\gamma \mathrm{H} 2 \mathrm{AX}$ assay was applied to quantify the genotoxicity of the PAs lasiocarpine and riddelliine. Several studies reported in the literature have assessed the in vitro genotoxicity of PAs using diverse assays, showing that PAs may induce divergent DNA damage including DNA-adducts, DNA cross-linking and clastogenic effects (Chan et al., 1994; Xia et al., 2006; Kim et al., 1995; Takanashi et al., 1980). The phosphorylation of histone H2AX ( $\gamma \mathrm{H} 2 \mathrm{AX})$ occurs after a DNA double strand break in a cell and reflects a global genotoxic insult that may originate from different types of DNA damage (Sedelnikova et al., 2010). These genotoxic effects may result in gene mutations and tumour formation (Chen et al., 2010; Hoeijmakers, 2009). When comparing the values obtained for in vivo genotoxicity of lasicarpine and riddelliine with PoDs derived from available tumour data. From data on the incidence of liver haemangiosarcoma obtained in 2-year carcinogenicity studies in rats upon chronic oral exposure to lasiocarpine and riddelliine (NTP, 1978, 2003). EFSA derived a BMD 10 of $0.131 \mathrm{mg} / \mathrm{kg} \mathrm{bw} /$ day for lasiocarpine and of $0.292 \mathrm{mg} / \mathrm{kg}$ bw/day for riddelliine (EFSA, 2017). These $\mathrm{BMD}_{10}$ values obtained from the 2-year carcinogenicity studies are one to two orders of magnitude lower than the $\mathrm{BMD}_{10}$ levels predicted in the present study and the dose levels used in other studies to detect genotoxicity (Chan et al., 1994; Xia et al., 2013; Yan et al., 2002). This may be related to the fact that the exposure duration in the genotoxicity studies is far shorter (1-5 days) than the 2-year exposure applied in carcinogenicity studies. Although the developed PBK models can predict the genotoxicity in vivo, which takes the modelling approach one step beyond the kinetics for bioactivation and closer to the ultimate adverse effect of tumour formation, a real challenge is to build models for prediction of tumour formation. The Moolgavkar-Venzon-Knudson (MVK) multistage carcinogenesis model can describe the tumour formation via mathematic formulas (Luebeck and Moolgavkar, 2002). The principle of this model is based on tumour formation being a multistep process, in which the normal cells become tumour cells upon a series of transformations induced by 
sequential mutations in different tumour suppressor genes and/or oncogenes. This has been investigated for colorectal cancer where mutations in the adenomatous polyposis coli (APC) tumour suppressor gene have been linked to early stages in the carcinogenic process, and where mutation in the K-ras oncogene and the P53 tumour suppressor gene are aligned with malignant transformations that occur later in the carcinogenic process (Frank, 2007). Such MVK model for tumour progression may be used to identify the adequate in vitro model(s) to provide the in vitro data for the PBK modelling based facilitated reverse dosimetry facilitated QIVIVE for prediction of in vivo carcinogenicity. However, so far, there are no in vitro experimental methods available for detection of cell death, cell division and mutation rates in different tumour stages and the effects of chemicals on these rates, while these parameters would be important for development of such QIVIVE approaches for carcinogenicity. Another step to be considered in these models for tumour formation would be repeated dose regimens. In Chapter 6, primary rat hepatocytes were used as the rat cell-based liver model to obtain in vitro data for prediction of acute liver toxicity in the rat in vivo. However, primary rat hepatocytes are difficult to maintain in culture for more than a few days, and this makes the cells inadequate for evaluating the chronic effects of chemicals at physiologically relevant (low) dose levels. To date, use of microfluidic platforms for the co-culture of primary rat hepatocytes and endothelial cells (Kang et al., 2015) and the HepatoPac culture technique (Ukairo et al., 2013) maybe an option for studying chronic exposure of PAs since primary rat hepatocytes can be cultured for at least 4 weeks with constant metabolic enzyme activities. Furthermore, the gene and protein expression could be included in in silico models. For example PAs have been shown to significant change Fez1 and Mgat5 genes that are related to cancer induction (Guo et al., 2007) and these results may illustrate the cellular network response that should be considered to enable definition of a comprehensive simulation model of chemical carcinogenicity.

\section{Extrapolation of the in vitro dose metrics to the in vivo situation}

Another important issue when translating in vitro concentration-response curves to in vivo doseresponse curves is the choice of the dose metric for the extrapolation. The predictions can be done based on two approaches. In the first approach, each in vitro concentration is set equal to the maximum concentration $\left(\mathrm{C}_{\max }\right)$ of the compound as input in the PBK model to calculate the oral dose that results in this concentration. In the second approach, each concentration is translated to an area under the time-response curve (AUC), by multiplying the concentration with the assay time (Daston et al., 2010). The choice of the dose metric to be used for the reverse dosimetry, $C_{\max }$ or AUC, depends on the mode of action of the test compound and the endpoint of interest. In Chapter 3, we translated the in vitro concentration-response curves for cytotoxicity to in vivo dose-response curves based on a $\mathrm{C}_{\max }$ approach, because for liver toxicity a threshold can be assigned and the effect size can be assumed to be dominated by the $\mathrm{C}_{\max }$ rather than by the AUC. The results obtained in this way for the predicted PoD of lasiocarpine were in line with the in vivo liver toxicity data. When making the 
the prediction based on the AUC approach, the predicted PoD for lasiocarpine was $111-173 \mathrm{mg} / \mathrm{kg}$ bw/day, more than one order of magnitude above the PoD derived from the in vivo liver toxicity data. This indicates that when predicting toxicity for which a threshold exists and thus a concentration below which there will be no effect, $\mathrm{C}_{\max }$ may be the selected parameter for the reverse dosimetry. In order to predict genotoxicity of lasiocarpine and riddelliine in rat, we translated the in vitro concentration-response curves of genotoxicity to in vivo dose-response curves based on the AUC approach (Chapter 6). When using $\mathrm{C}_{\max }$ for this conversion, the predicted $\mathrm{BMD}_{10}$ of lasiocarpine and riddelliine was up to 17.4- and 42.2-fold lower compared to in vivo animal data. However, when predicting $\mathrm{BMD}_{10}$ values for lasiocarpine and riddelliine using the AUC approach, for lasiocarpine the difference between predicted PoD values and the observed data derived from an in vivo study detecting DNA adduct formation was only 1.1-fold (Xia et al., 2013). For riddelliine, the predicted PoD was 1.8- to 2.9-fold different from the in vivo dose causing detectable DNA-adduct formation (Xia et al., 2013; Yan et al., 2002), and 3.4-fold different from the in vivo dose causing detectable unscheduled DNA synthesis in rat (Chan et al., 1994). This thesis confirmed that when considering non-threshold effects that accumulate in time, including effects like genotoxicity and carcinogenicity, use of the AUC is preferred over use of the $C_{\max }$ in reverse dosimetry (Groothuis et al., 2015).

\section{Future perspectives}

\section{Development of generic PBK models for a group of compounds}

Due to the lack of available in vivo liver toxicity, genotoxicity and carcinogenicity data for PAs, Chapter 4, 5 and 6 investigated whether using PBK modelling-based reverse dosimetry can predict PoDs for lasiocarpine and riddelliine by translating in vitro cytotoxicity and genotoxicity concentration-response curves to in vivo dose-response curves. To this end, PBK models were developed for lasiocarpine and riddelliine, respectively. However, it is important to realise that the PBK models developed in the present thesis specifically for these two PAs required a large number of parameters. Therefore, development of such PBK models is time consuming although compared to performing an animal bioassay the amount of work may still be considered relatively small, cheaper and of less ethical concern. Future efforts could be focussed on developing more generic PBK models for the PAs. This will provide an even more efficient approach for future risk assessment of groups of genotoxic carcinogens for which rodent toxicity data are not available (Bessems et al., 2014). Some efforts in development of generic PBK models have been reported. Zhang et al. (2017) developed a generic PBK model that can predict in vivo uterotrophic responses induced by $17 \beta$-estradiol (E2) and bisphenol A (BPA) in rats, and this work could serve as a starting point of developing a generic PBK model to predict the estrogenicity for a large number of chemicals (Zhang et al., 2017). Brightman et al. (2007) applied a generic PBK model that can estimate the in vivo pharmacokinetics of a set of 18 compounds for which plasma levels have been determined following an intravenous dose (Brightman et al., 2006). Depending on the group of chemicals under study only a few compound specific 
parameters may have to be added but the PBK model development could start from the generic basic model and its evaluation before adding additional parameters. In order to develop the model more efficient, some parameter may be obtained from in silico methods instead of experimental work. For example, the sensitivity analysis showed that permeability coefficients $\left(P_{\text {app }}\right)$ had a large influence on model prediction (Chapter 3). Use of experimental Caco-2 transport studies to derive the $P_{\text {app }}$ values in PBK models has been reported (Louisse et al., 2015; Li et al., 2017; Zhang et al., 2018). To build a generic PBK model for a large number of chemicals, such parameters could be obtained from in silico methods. Hou et al. (2004) described that $P_{\text {app }}$ values for Caco-2 transport can be predicted using QSAR approaches requiring information on the polar surface area alone (Hou et al., 2004), indicating that no experimental work is needed to quantify this parameter. Consequently, development of a generic PBK models can assist in making the PBK model description more time and cost efficient.

\section{Quality control of botanicals}

Since high level of PAs have been detected in botanicals and botanical preparations (Bodi et al., 2014; IPCS, 1988; Mulder et al., 2015, 2018; EFSA, 2016), and these botanical products are still commercially available, this situation raises a concern for public health and represents a food safety issue. In spite of this, consumer demands for these products continue to grow. Meantime, the reported cases of adverse events upon consumption of botanicals and botanical preparations are also rising globally (Rocha et al., 2016). To adequately guarantee the safe use of botanical preparations, it is essential to internationally harmonize the approach and procedures for the regulatory safety assessment of those products. In reality, however, the legislation and the risk assessment criteria for botanical supplements vary among different countries (Low et al., 2017). While some countries classify PFS as a food or health food (CFDA, 2016; JETRO, 2011), others regulate PFS as medicines (Health Canada 2016; TGA, 2013). To date, EU, Australia and the US have established criteria for the safety evaluation of PFS ingredients (EFSA, 2009) whereas there appear to be no such criteria in other countries. Internet sales of herbal products has further complicated matters as consumers can easily purchase products from all over the world. To address these issues and ensure public health, increasing regulatory oversight for botanical preparations seems necessary. Harmonization of risk assessment criteria as well as the creation of internationally recognized compendia of botanicals should be emphasized as a way forward. The international regulatory community may create a list of botanicals with known safety concerns and update the list on a regular basis, such as is the case for the compendium developed by EFSA (EFSA, 2012). Also a better system for quality control and traceability seems indicated. The traceability system can be built for each botanical product, food business operator, including those trading in food supplements. The data and information to be collected include name, address and date of the supplier, business customer, delivery company and food business operator. The most important information about the batch should always follow the material by a batch documentation, and integrity of botanical material (EUROPAM 2006; 
FoodSupplementsEurope 2016). The findings of widespread contamination by PAs in herbal teas has confirmed that the situation with PA contamination is serious. Applying a good quality control can prevent the herbal tea contamination by PA-producing plants. A Code of Practice (CoP) has been developed that focuses on weed control and provides guidance on good management practices to prevent and reduce PA contamination by control measures for the management of PA-producing plants (CAC, 2014). In addition, an appropriate sampling plan should be developed depending on whether a herbal substance (spot contamination) or a herbal preparation/finished product (homogenous sample) is tested. Sampling should be in accordance with Commission Regulation 401/2006/EC (EC, 2006). Manufacturers should be required to provide ingredient information and highly sensitive analytical methods are also needed to monitor the PA levels during the sampling and production process. These quality control measures will facilitate risk assessment and guarantee the safety of the herbal products.

\section{Conclusion}

This thesis assessed the risks of herbal teas, herbal medicines and PFS that contain PAs based on the MOE approach, and also developed new methods to tackle existing data gaps and to improve the scientific basis for PA risk and safety assessment. The overall conclusion of the risk assessment of herbal products revealed that daily life-time consumption of some of these products would be a priority for risk management. When considering realistic exposure scenarios, exposure to most of these herbal products would have MOE values higher than 10000 indicating a low priority for risk management. Moreover, this thesis demonstrated that the combined in vitro PBK modelling-based reverse dosimetry approach could adequately predict in vivo liver toxicity and genotoxicity for two model PAs, lasiocarpine and riddelliine. Such QIVIVE methods may prove to be of use in defining more realistic REP values for the different food/feed-related PAs, i.e. REP values that are not solely based on in vitro concentration-effect data but also taking differences in toxicokinetics into account. The results obtained reveal the feasibility of this combined quantitative in vitro-in silico approach to determine a PoD for a chemical without the use of experimental animals and to address the issue of how to use in vitro data for risk assessment. 


\section{Reference}

Allemang, A., Mahony, C., Lester, C. and Pfuhler, S., 2018. Relative potency of fifteen pyrrolizidine alkaloids to induce DNA damage as measured by micronucleus induction in HepaRG human liver cells. Food and chemical toxicology, 121, pp.72-81.

Bell, C.C., Hendriks, D.F., Moro, S.M., Ellis, E., Walsh, J., Renblom, A., Puigvert, L.F., Dankers, A.C., Jacobs, F., Snoeys, J. and Sison-Young, R.L., 2016. Characterization of primary human hepatocyte spheroids as a model system for drug-induced liver injury, liver function and disease. Scientific reports, 6, p.25187.

Bessems JG, Loizou G, Krishnan K, Clewell III HJ, Bernasconi C, Bois F, Coecke S, Collnot EM, Diembeck W, Farcal LR, Geraets L. PBTK modelling platforms and parameter estimation tools to enable animal-free risk assessment: recommendations from a joint EPAA-EURL ECVAM ADME workshop. Regulatory Toxicology and Pharmacology. 2014 Feb 1;68(1):119-39.

Brightman FA, Leahy DE, Searle GE, Thomas S. Application of a generic physiologically based pharmacokinetic model to the estimation of xenobiotic levels in human plasma. Drug metabolism and disposition. 2006 Jan 1;34(1):94-101.

Bodi, D., Ronczka, S., Gottschalk, C., Behr, N., Skibba, A., Wagner, M., Lahrssen- Wiederholt, M., Preiss-Weigert, A., These, A., 2014. Determination of pyrrolizidine alkaloids in tea, herbal drugs and honey. Food Addit. Contam. Part A 31, 1886-1895.

COC, 2012. Committee on Carcinogenicity of Chemicals in Food, Consumer Products and theEnvironment

Chan, P.C., Mahler, J., Bucher, J.R., Travlos, G.S. and Reid, J.B., 1994. Toxicity and carcinogenicity of riddelliine following 13 weeks of treatment to rats and mice. Toxicon, 32(8), pp.891-908.

Chen, T., Mei, N., Fu, P.P., 2010. Genotoxicity of pyrrolizidine alkaloids. Journal of Applied Toxicology: An International Journal 30, 183-196.

Crump, K., Hoel, D., Langley, C., Peto, R., 1976. Fundamental carcinogenic processes and their implications for low dose risk assessment. Cancer research 36, 2973-2979.

Coecke, S., Pelkonen, O., Leite, S.B., Bernauer, U., Bessems, J.G.M., Bois, F.Y., Gundert- Remy, U., Loizou, G., Testai, E., Zaldivar, J.M., 2013. Toxicokinetics as a key to the integrated toxicity risk assessment based primarily on non-animal approaches. Toxicol. In Vitro 27, 1570-1577.

CAC, 2014. Code of practice for weed control to prevent and reduce pyrrolizidine alkaloid contamination in food and feed. Rome: Codex Alimentarius Commission Secretariat.

CFDA. 2016. "Health food registration and record management approach" (State Food and Drug Administration Order No. 22).

Dalefield, R.R., Gosse, M.A., Mueller, U., 2016. A 28-day oral toxicity study of echimidine and lasiocarpine in Wistar rats. Regul Toxicol Pharmacol. 81, 146-154.

Daston, G.P., Chapin, R.E., Scialli, A.R., Piersma, A.H., Carney, E.W., Rogers, J.M. and Friedman, J.M., 2010. A different approach to validating screening assays for developmental toxicity. Birth Defects Research Part B: Developmental and Reproductive Toxicology, 89(6), pp.526530.

Duringer, J. M., Buhler, D. R., and Craig, A. M. (2004) Comparison of hepatic in vitro metabolism of the pyrrolizidine alkaloid senecionine in sheep and cattle. Am. J. Vet. Res. 65, 1563-1572.

EFSA, 2011. Scientific Opinion on Pyrrolizidine alkaloids in food and feed. EFSA J. 9.

EFSA, 2005. Opinion of the Scientific Committee on a Request from EFSA Related to a Harmonised Approach for Risk Assessment of Sub-stances which Are Both Genotoxic and Carcinogenic. EFSA J. 282, 1-31.

EFSA, 2016. Dietary exposure assessment to pyrrolizidine alkaloids in the European population. EFSA J. 14. Available at: http://onlinelibrary.wiley.com/doi/10. 2903/j.efsa.2016.4572/epdf.

EFSA, 2017. Risks for human health related to the presence of pyrrolizidine alkaloids in honey, tea, herbal infusions and food supplements. EFSA Journal 15.

EUROPAM, 2006. Guidelines for Good Agricultural and Wild Collection Practice (GACP) of Medicinal and Aromatic Plants

EFSA, 2012. Compendium of botanicals reported to contain naturally occuring substances of possible concern for human health when used in food and food supplements. EFSA Journal, 10(5), p.2663. 
EFSA, 2009. Guidance on safety assessment of botanicals and botanical preparations intended for use as ingredients in food supplements. EFSA Journal, 7(9), p.1249.

EC, 2006. Commission Regulation EC n. 401/2006 of 23 February. Laying down the methods of sampling and analysis for the official control of the levels of mycotoxins in foodstuffs. Official Journal of the European Union, London, 9 Mar. 2006. L. 70, p. 12-34.

Fu, P.P., Xia, Q., Lin, G., Chou, M.W., 2004. Pyrrolizidine alkaloids-genotoxicity, metabolism enzymes, metabolic activation, and mechanisms. Drug metabolism reviews 36, 1-55.

Fashe, M.M., Juvonen, R.O., Petsalo, A., Räsänen, J. and Pasanen, M., 2015. Species-specific differences in the in vitro metabolism of lasiocarpine. Chemical research in toxicology, 28(10), pp.2034-2044.

FoodSupplementsEurope, 2016. Quality of botanical preparations specific recommendations for the manufacturing of botanical preparations, including extracts as food supplements.

Frank, S.A., 2007. Dynamics of cancer: incidence, inheritance, and evolution. Princeton University Press.

Guillouzo, A., Corlu, A., Aninat, C., Glaise, D., Morel, F., Guguen-Guillouzo, C., 2007. The human hepatoma HepaRG cells: a highly differentiated model for studies of liver metabolism and toxicity of xenobiotics. Chemico-biological interactions 168, 66-73.

Gerets HHJ, Tilmant K, Gerin B, Chanteux H, Depelchin BO, Dhalluin S, Atienzar FA (2012) Characterization of primary human hepatocytes, HepG2 cells, and HepaRG cells at the mRNA level and CYP activity in response to inducers and their predictivity for the detection of human hepatotoxins. Cell Biology and Toxicology 28(2):69-87

Guo, L., Mei, N., Dial, S., Fuscoe, J. and Chen, T., 2007, December. Comparison of gene expression profiles altered by comfrey and riddelliine in rat liver. BioMed Central 8, S22.

Groothuis, F.A., Heringa, M.B., Nicol, B., Hermens, J.L., Blaauboer, B.J. and Kramer, N.I., 2015. Dose metric considerations in in vitro assays to improve quantitative in vitro-in vivo dose extrapolations. Toxicology, 332, pp.30-40.

Hoeijmakers, J.H., 2009. DNA damage, aging, and cancer. New England Journal of Medicine 361, 1475-1485.

Huan, J. Y., Miranda, C. L., Buhler, D. R., and Cheeke, P. R. (1998) Species differences in the hepatic microsomal enzyme metabolism of the pyrrolizidine alkaloids. Toxicol. Lett. 99, 127-137.

Hou, T.J., Zhang, W., Xia, K., Qiao, X.B., Xu, X.J., 2004. ADME evaluation in drug discovery. 5. Correlation of Caco-2 permeation with simple molecular properties. J Chem Inf Comput Sci. 44, 1585-1600.

Health Canada. 2016. About natural health product regulations in Canada - Health Canada.

IPCS, 2005. Chemical-specific adjustment factors for interspecies differences and human variability: Guidance document for use of data in dose/concentration-response assessment. World Health Organization Geneva.

IPCS, 1988. International Programme on Chemical Safety Health and Safety Environmental Health Criteria 80: Pyrrolizidine Alkaloids.

Jago, M.V., 1970. A method for the assessment of the chronic hepatoxicity of pyrrolizidine alkaloids. Immunol Cell Biol. 48(1), p.93.

JETRO. 2011. Guidebook for export to Japan (Food Articles) $2011<$ Health foods and dietary supplements $>$ March, 2011.

Kvist AJ, Kanebratt KP, Walentinsson A, Palmgren H, O'Hara M, Bjorkbom A, Andersson LC, Ahlqvist M, Andersson TB., 2018. Critical differences in drug metabolic properties of human hepatic cellular models, including primary human hepatocytes, stem cell derived hepatocytes, and hepatoma cell lines. Biochem Pharmacol 155:124-140

Knöspel, F., Jacobs, F., Freyer, N., Damm, G., De Bondt, A., van den Wyngaert, I., Snoeys, J., Monshouwer, M., Richter, M., Strahl, N. and Seehofer, D., 2016. In vitro model for hepatotoxicity studies based on primary human hepatocyte cultivation in a perfused 3D bioreactor system. International journal of molecular sciences, 17(4), p.584.

Kimura, H., Sakai, Y. and Fujii, T., 2018. Organ/body-on-a-chip based on microfluidic technology for drug discovery. Drug metabolism and pharmacokinetics, 33(1), pp.43-48. 
Kim, H.Y., Stermitz, F.R. and Coulombe Jr, R.A., 1995. Pyrrolizidine alkaloid-induced DNA-protein cross-links. Carcinogenesis, 16(11), pp.2691-2697.

Kang, Y.B., Sodunke, T.R., Lamontagne, J., Cirillo, J., Rajiv, C., Bouchard, M.J. and Noh, M., 2015. Liver sinusoid on a chip: Long - term layered co - culture of primary rat hepatocytes and endothelial cells in microfluidic platforms. Biotechnology and bioengineering, 112(12), pp.2571-2582.

Lin, G., Cui, Y.-Y., Liu, X.-Q., 2003. Gender differences in microsomal metabolic activation of hepatotoxic clivorine in rat. Chemical research in toxicology 16, 768-774.

Lübberstedt, M., Müller-Vieira, U., Mayer, M., Biemel, K.M., Knöspel, F., Knobeloch, D., Nüssler, A.K., Gerlach, J.C. and Zeilinger, K., 2011. HepaRG human hepatic cell line utility as a surrogate for primary human hepatocytes in drug metabolism assessment in vitro. Journal of pharmacological and toxicological methods, 63(1), pp.59-68.

LeCluyse, E.L., 2001. Human hepatocyte culture systems for the in vitro evaluation of cytochrome P450 expression and regulation. European journal of pharmaceutical sciences, 13(4), pp.343368.

Luebeck, E.G. and Moolgavkar, S.H., 2002. Multistage carcinogenesis and the incidence of colorectal cancer. Proceedings of the National Academy of Sciences, 99(23), pp.15095-15100.

Li, H., Zhang, M., Vervoort, J., Rietjens, I.M., van Ravenzwaay, B. and Louisse, J., 2017. Use of physiologically based kinetic modeling-facilitated reverse dosimetry of in vitro toxicity data for prediction of in vivo developmental toxicity of tebuconazole in rats. Toxicology letters, 266, pp.85-93.

Louisse, J., Bosgra, S., Blaauboer, B.J., Rietjens, I.M. and Verwei, M., 2015. Prediction of in vivo developmental toxicity of all-trans-retinoic acid based on in vitro toxicity data and in silico physiologically based kinetic modeling. Archives of toxicology, 89(7), pp.1135-1148.

Low, T.Y., Wong, K.O., Yap, A.L., De Haan, L.H. and Rietjens, I.M., 2017. The regulatory framework across international jurisdictions for risks associated with consumption of botanical food supplements. Comprehensive Reviews in Food Science and Food Safety, 16(5), pp.821834.

Mulder, P.P., Sanchez, P.L., These, A., Preiss-Weigert, A., 2015. Occurrence of Pyrrolizidine Alkaloids in Food, vol. 12. EFSA Supporting Publications.

Mulder PP, López P, Castelari M, Bodi D, Ronczka S, Preiss-Weigert A, These A. Occurrence of pyrrolizidine alkaloids in animal-and plant-derived food: results of a survey across Europe. Food Additives \& Contaminants: Part A. 2018 Jan 2;35(1):118-33.

Merz, K.H. and Schrenk, D., 2016. Interim relative potency factors for the toxicological risk assessment of pyrrolizidine alkaloids in food and herbal medicines. Toxicology letters, 263, pp.44-57.

Nolan, J.P., Scheig, R.L., Klatskin, G., 1966. Delayed hepatitis and cirrhosis in weanling rats following a single small dose of the senecio alkaloid, lasiocarpine. AM J PATHOL. 49, 129151.

NTP, 1978. Bioassay of lasiocarpine for possible carcinogenicity. National Cancer Institute carcinogenesis technical report series 39,1 .

NTP, 2003. Toxicology and carcinogenesis studies of riddelliine (CAS No. 23246-96-0) in F344/N rats and B6C3F1 mice (gavage studies). National Toxicology Program technical report series, 1.

O’Brien J, Renwick AG, Constable A, Dybing E, Müller DJ, Schlatter J, Slob W, Tueting W, Van Benthem J, Williams GM, Wolfreys A. Approaches to the risk assessment of genotoxic carcinogens in food: a critical appraisal. Food and Chemical Toxicology. 2006 Oct 1;44(10):1613-35.

Prakash, A.S., Pereira, T.N., Reilly, P.E., Seawright, A.A., 1999. Pyrrolizidine alkaloids in human diet. Mutation Research/Genetic Toxicology and Environmental Mutagenesis 443, 53-6.

Roeder, E., 2000. Medicinal plants in China containing pyrrolizidine alkaloids. Pharmazie 55, 711-726.

Rocha, T., Amaral, J.S. and Oliveira, M.B.P., 2016. Adulteration of dietary supplements by the illegal addition of synthetic drugs: a review. Comprehensive reviews in food science and food safety, 15(1), pp.43-62. 
Sedelnikova, O.A., Redon, C.E., Dickey, J.S., Nakamura, A.J., Georgakilas, A.G., Bonner, W.M., 2010. Role of oxidatively induced DNA lesions in human pathogenesis. Mutation Research/Reviews in Mutation Research 704, 152-159.

Shimada, T., Yamazaki, H., Mimura, M., Inui, Y., Guengerich, F.P., 1994. Interindividual variations in human liver cytochrome P-450 enzymes involved in the oxidation of drugs, carcinogens and toxic chemicals: studies with liver microsomes of 30 Japanese and 30 Caucasians. Journal of Pharmacology and Experimental Therapeutics 270, 414-423.

Stegelmeier, B. L., Edgar, J. A., Colegate, S. M., Gardner, D. R., Schoch, T. K., Coulombe, R. A., and Molyneux, R. J. 1999. Pyrrolizidine alkaloid plants, metabolism and toxicity. J. Nat. Toxins 8, 95-116.

Turpeinen, M., Tolonen, A., Chesne, C., Guillouzo, A., Uusitalo, J., Pelkonen, O., 2009. Functional expression, inhibition and induction of CYP enzymes in HepaRG cells. Toxicology in Vitro 23, 748-753.7.

Takanashi, H., Umeda, M. and Hirono, I., 1980. Chromosomal aberrations and mutation in cultured mammalian cells induced by pyrrolizidine alkaloids. Mutation Research/Genetic Toxicology, 78(1), pp.67-77. TGA. 2013. An overview of the regulation of complementary medicines in Australia.

TGA, 2013. Registered complementary medicines | Therapeutic Goods Administration (TGA).

Ukairo, O., Kanchagar, C., Moore, A., Shi, J., Gaffney, J., Aoyama, S., Rose, K., Krzyzewski, S., McGeehan, J., Andersen, M.E. and Khetani, S.R., 2013. Long - term stability of primary rat hepatocytes in micropatterned cocultures. Journal of biochemical and molecular toxicology, 27(3), pp.204-212.

Wong, M.W., Pridgeon, C.S., Schlott, C., Park, B.K. and Goldring, C.E., 2018. Status and Use of Induced Pluripotent Stem Cells (iPSCs) in Toxicity Testing. In Drug-Induced Liver Toxicity (pp. 199-212). Humana Press, New York, NY.

Winter, C. K., Segall, H. J., and Jones, A. D., 1988. Species differences in the hepatic microsomal metabolism of the pyrrolizidine alkaloid senecionine. Comp. Biochem. Physiol., C: Comp. Pharmacol. 90,429-433.

Xia, Q., Zhao, Y., Von Tungeln, L.S., Doerge, D.R., Lin, G., Cai, L., Fu, P.P., 2013. Pyrrolizidine alkaloid-derived DNA adducts as a common biological biomarker of pyrrolizidine alkaloidinduced tumorigenicity. Chemical research in toxicology 26, 1384-1396.

Xia, Q., Chou, M.W., Edgar, J.A., Doerge, D.R., Fu, P.P., 2006. Formation of DHP-derived DNA adducts from metabolic activation of the prototype heliotridine-type pyrrolizidine alkaloid, lasiocarpine. Cancer letters 231, 138-145.

Yan, J., Nichols, J., Yang, Y.-C., Fu, P.P., Chou, M.W., 2002. Detection of riddelliine-derived DNA adducts in blood of rats fed riddelliine. International Journal of Molecular Sciences 3, 10191026.

Zhang M, van Ravenzwaay B, Fabian E, Rietjens IM, Louisse J. Towards a generic physiologically based kinetic model to predict in vivo uterotrophic responses in rats by reverse dosimetry of in vitro estrogenicity data. Archives of toxicology. 2018 Mar 1;92(3):1075-88.

Zuo, R., Li, F., Parikh, S., Cao, L., Cooper, K.L., Hong, Y., Liu, J., Faris, R.A., Li, D., Wang, H., 2017. Evaluation of a Novel Renewable Hepatic Cell Model for Prediction of Clinical CYP3A4 Induction Using a Correlation-Based RIS Approach. Drug Metabolism and Disposition, dmd. 116.072124 . 

Appendices 


\section{Summary}

Botanicals and botanical preparations may contain natural constituents that are of concern for human health. One group of such natural toxic compounds that may raise a concern is the group of pyrrolizidine alkaloids (PAs). At the current state-of-the-art, risk assessment of botanicals and botanical preparations is generally not required before they can enter the market. The present thesis aimed to perform the risk assessment for PAs derived from botanical products following daily lifetime exposure and also more realistic exposure scenarios. Another aim of the present thesis was to investigate whether animal-free testing strategies could be of use in tackling data and knowledge gaps for PAs by predicting in vivo toxicity of different PAs and whether proofs-of-principle for applying such alternative testing approaches could be provided for two selected PA model compounds, riddelliine and lasiocarpine.

Chapter 1 provided general background information on PAs, a description of the physico-chemical properties of lasiocarpine and riddelliine, as well as exposure, metabolism, genotoxicity, carcinogenicity and hepatotoxicity characteristics of PAs, a brief outline of the methodology used for risk assessment of PAs, a short introduction to PBK modelling-based reverse dosimetry and the objectives of the present thesis. In Chapter 2, a risk assessment was performed based on the margin of exposure (MOE) approach using the BMDL10 (benchmark dose lower confidence limit for $10 \%$ extra risk on tumour formation above background levels) of $0.07 \mathrm{mg} / \mathrm{kg}$ bw/day for lasiocarpine and mean levels of PAs reported in literature for herbal teas and plant food supplements (PFS). After using interim REP (relative potency) factors to 'adjust' PA levels and resulting exposure, 54\% of the types of herbal teas and $14 \%$ of the PFS were estimated to result in daily intakes that would give rise to MOE values lower than 10000 , indicating a possible health risk. The herbal preparations that raised a concern included preparations containing PA-producing plants but also preparations containing nonPA-producing plants. In Chapter 3, the MOE approach, using the BMDL 10 of $0.24 \mathrm{mg} / \mathrm{kg}$ bw/day for riddelliine and total PA levels, and Haber's rule were employed to analyse the risks of shorter-thanlifetime exposure for 5 herbal teas, 8 herbal medicines and previously analysed herbal products from Chapter 2. This revealed that shorter-than-lifetime use of these herbal products resulted in MOE values lower than 10000 when the preparations would be used for only 40 to up to 3450 weeks during a lifetime (depending on the preparation), with for only a limited number of herbal teas and medicines use of two weeks a year (150 weeks during a 75 year lifetime) still raising a concern. The results also revealed that the PA levels extracted from intact leaves were lower than the leaves extracted from comminuted leaves, and this may influence the actual exposure and corresponding risk assessment. In Chapter 4 and 5, PBK models were developed for prediction of liver toxicity of lasiocarpine and riddelliine in rats and humans on the basis of in vitro toxicity data. Using PBK model based reverse dosimetry the in vitro concentration-response curves for cytotoxicity in primary rat hepatocytes were translated to in vivo dose-response curves. From these in vivo dose-response curves, the predicted 
$\mathrm{BMDL}_{5}-\mathrm{BMDU}_{5}$ (lower/upper limit of the $90 \%$ confidence interval of the benchmark dose that gives a $5 \%$ response) of lasiocarpine and riddelliine in both rats (Chapter 4) and humans (Chapter 5) were obtained. Due to the absence of in vivo acute liver toxicity data reported in the literature for riddelliine, the predicted PoDs (point of departures) for in vivo liver toxicity could only be evaluated for lasiocarpine. To this end, the predicted $\mathrm{BMDL}_{5}-\mathrm{BMDU}_{5}$ of lasiocarpine for rats was compared with in vivo reported data for acute liver toxicity of lasiocarpine, and the results showed that the predicted $\mathrm{BMDL}_{5}-\mathrm{BMDU}_{5}$ of lasiocarpine appeared to fall well within the range of PoDs obtained from the available in vivo studies. In Chapter 6, the same approach was used for prediction of genotoxicity for lasiocarpine and riddelliine in rats. The in vitro concentration-genotoxic response curves of the two PAs, as determined in primary rat hepatocytes using the $\gamma \mathrm{H} 2 \mathrm{AX}$ assay, were translated into in vivo dose-response curves by applying the developed PBK models. The predicted $\mathrm{BMD}_{10}$ (benchmark dose that gives a $10 \%$ response) values for lasiocarpine and riddelliine were in line with the experimental in vivo genotoxicity data available in the literature. Chapter 7 presented a summary and overall discussion of the results obtained in the thesis, and concluded with the future perspectives regarding the development and application of generic PBK models and the safety assessment of botanical preparations.

Altogether, the present thesis developed new methods to support and improve the risk assessment of PAs without the use of experimental animals, and demonstrated the feasibility and importance of combined in vitro PBK modelling-based reverse dosimetry to adequately predict in vivo acute liver toxicity and genotoxicity using lasiocarpine and riddelliine as two model PAs. 


\section{Acknowledgments}

The long journey of my PhD study ends, and I see this end with inner peace. Before the next adventure starts, I must express my sincere gratitude for all support that I have got during my $\mathrm{PhD}$ journey.

I would like to give my greatest thanks to my promotor Prof. Dr. I.M.C.M. Rietjens, it is your admittance letter being the key to my successful application for a grant from the Chinese Scholarship Council (CSC), making the very first step of my PhD journey. I would like to greatly thank you for every efforts you spent on the training of my critical thinking and independence of studying. Your structural suggestions and novel ideas gave me inspirations, encouraging me to get into my $\mathrm{PhD}$ project with increasing interest.

I am highly grateful for the financial support from CSC, which allows me to focus on my PhD study without living concern in the Netherlands.

I would like to specially thank my co-promoter Dr A.A.C.M. Peijnenburg, thank you for your critical comments, which inspired me to dive into deeper levels in science field. I would like to highly thank Patrick P.J. Mulder for your valuable suggestions with my publications. All of you were really helpful! Because of your support, I can do my $\mathrm{PhD}$ thesis smoothly. Now, it is time to give my thanking words and a huge hug to all of you, Aziza, Mebrahtom, Artem, Marta, Suparmi, Ashraf, Kornphimol, Diego, Mengying, Jia, Jing, Lenny, Diana, Georgia, Shuo, Ixchel, Miaoying, Kathia, Annelies, Felicia, Isaac, Shengsheng, Qianrui, Bohan, Biyao and Ignacio. I also would like sincerely to thank Lidy, Sebas, Hans, Bert and Laura for the support.

A big thank you goes to my paranymphs, Georgia and Shuo, and things you have done for me. I really enjoyed the time we spent together during the last four years.

I would like to express my best sincere gratitude to my family. Mom, dad and my sister, thank you so much for everything you've done for me. Every support, encouragement and care from you are always the most precious, selfless gift for me. Brother Biao, maybe it is a bit too formal to say thank you for your close company, but I wish that we can hold this close company till the end of time. 


\section{Curriculum Vitae}

Lu Chen was born on September 24th, 1988 in Sichuan province, China. She completed her BSc in Computer Science at Chongqing Three Gorges University, China. Following the accomplishment of her BSc study in 2012, she did her MSc study in Animal Nutrition and Feed Science at the Institute of Animal Sciences of Chinese Academy of Agricultural Sciences (CAAS). During the MSc period, she focused on the improvement of the quality for dairy products. She obtained her MSc diploma in July, 2015. On May of 2015, she was granted a scholarship from the Chinese Scholarship Council, which contributed to the successful application for a $\mathrm{PhD}$ position in Toxicology Group at Wageningen University. In Toxicology, she has performed all the experiments described in this thesis from October 2015 till September 2019. 


\section{List of publications}

1. Chen, L., Mulder, P.P., Louisse, J., Peijnenburg, A., Wesseling, S. and Rietjens, I.M., 2017. Risk assessment for pyrrolizidine alkaloids detected in (herbal) teas and plant food supplements. Regulatory Toxicology and Pharmacology, 86, pp.292-302.

2. Chen, L., Ning, J., Louisse, J., Wesseling, S. and Rietjens, I.M., 2018. Use of physiologically based kinetic modelling-facilitated reverse dosimetry to convert in vitro cytotoxicity data to predicted in vivo liver toxicity of lasiocarpine and riddelliine in rat. Food and Chemical Toxicology, 116, pp.216-226.

3. Chen, L., Mulder, P.P., Peijnenburg, A. and Rietjens, I.M., 2019. Risk assessment of intake of pyrrolizidine alkaloids from herbal teas and medicines following realistic exposure scenarios. Food and Chemical Toxicology, 130, pp.142-153.

4. Chen, L., Peijnenburg, A., Laura, H. and Rietjens, I.M., 2019. Prediction of in vivo genotoxicity of lasiocarpine and riddelliine in rat liver using a combined in vitro-physiologically based kinetic modelling-facilitated reverse dosimetry approach. Archives of toxicology, pp:1-11.

5. Ning, J., Chen, L., Strikwold, M., Louisse, J., Wesseling, S. and Rietjens, I.M., 2019. Use of an in vitro-in silico testing strategy to predict inter-species and inter-ethnic human differences in liver toxicity of the pyrrolizidine alkaloids lasiocarpine and riddelliine. Archives of toxicology, pp.1-18. 


\section{Overview of completed training activities}

Discipline specific activities

Name

Organizer Year

Molecular toxicology

PET

2016

Pathobiology

PET

2016

Epidemiology

PET

2016

Introduction Laboratory Animal science

PET

2016

Organ toxicology

PET

2017

Risk assessment

PET

2017

Cell Toxicology

PET

2018

Immunotoxicology

PET

2018

Legal and regulatory toxicology

PET

2018

Mettings

Dutch Society for Toxicology (poster)

NVT

2017

pyrrolizidine alkaloids workshop

Kaiserslautern University

2018

General courses

Name

Organizer

Year

VLAG PhD week

VLAG

2016

WGS

2017

Project and time management

WGS

2016

Brain Training

WGS

2016

$\mathrm{PhD}$ peer consultation

WGS

2017

Information Literacy including Endnote

WGS

2017

Introduction

Reviewing a scientific paper

WGS

2016

Brain-friendly working \& writing

WGS

2018

Optional

Name

Preparation of research proposal

Follow the lecture of TOX

Weekly group meetings

Organizer

Year

Research group

2015

Research group

2015-2017

Research group

2015-2019 


\section{Colophon}

The research described in this thesis was financially supported by China Scholarship Council, China and Toxicology Group of Wageningen University, the Netherlands.

Financial support from the Toxicology Group of Wageningen University.

Layout: Lu Chen

Cover design: Lu Chen

Printing: GVO drukkers \& vormgevers B.V. Ede (NL)|gvo.nl 